\title{
Smoothelins: expression, regulation, and function
}

Citation for published version (APA):

Rensen, S. S. M. (2004). Smoothelins: expression, regulation, and function. [Doctoral Thesis, Maastricht University]. Ponsen \& Looijen B.V. https://doi.org/10.26481/dis.20040312sr

Document status and date:

Published: 01/01/2004

DOI:

10.26481/dis.20040312sr

Document Version:

Publisher's PDF, also known as Version of record

\section{Please check the document version of this publication:}

- A submitted manuscript is the version of the article upon submission and before peer-review. There can be important differences between the submitted version and the official published version of record.

People interested in the research are advised to contact the author for the final version of the publication, or visit the DOI to the publisher's website.

- The final author version and the galley proof are versions of the publication after peer review.

- The final published version features the final layout of the paper including the volume, issue and page numbers.

Link to publication

\footnotetext{
General rights rights.

- You may freely distribute the URL identifying the publication in the public portal. please follow below link for the End User Agreement:

www.umlib.nl/taverne-license

Take down policy

If you believe that this document breaches copyright please contact us at:

repository@maastrichtuniversity.nl

providing details and we will investigate your claim.
}

Copyright and moral rights for the publications made accessible in the public portal are retained by the authors and/or other copyright owners and it is a condition of accessing publications that users recognise and abide by the legal requirements associated with these

- Users may download and print one copy of any publication from the public portal for the purpose of private study or research.

- You may not further distribute the material or use it for any profit-making activity or commercial gain

If the publication is distributed under the terms of Article $25 \mathrm{fa}$ of the Dutch Copyright Act, indicated by the "Taverne" license above, 


\section{Smoothelins: expression, \\ regulation, and function}


The studies presented in this thesis were performed at the Department of Genetics and Cell Biology and the Department of Molecular Cell Biology and Genetics, Cardiovascular Research Institute Maastricht, Universiteit Maastricht, The Netherlands.

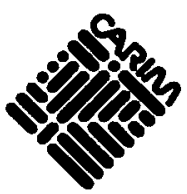

Printed by Grafisch bedrijf Ponsen \& Looijen B.V.

ISBN 90-6464-904-9

(c) Sander Rensen, Maastricht 2004 


\title{
Smoothelins: expression, \\ regulation, and function
}

\author{
Proefschrift \\ ter verkrijging van de graad van doctor \\ aan de Universiteit Maastricht, \\ op gezag van de Rector Magnificus, \\ Prof. mr. G.P.M.F. Mols \\ volgens het besluit van het College van Decanen, \\ in het openbaar te verdedigen \\ op vrijdag 12 maart 2004 om 14.00 uur \\ door \\ Sander Sebastiaan Maria Rensen \\ geboren te Deventer op 10 juni 1975
}


Promotor:

Prof. Dr. M.H. Hofker

Copromotores:

Dr. G.J.J.M. van Eys

Dr. P.A.F.M. Doevendans (HLCU/UMC - Utrecht)

Beoordelingscommissie:

Prof. Dr. H.A.J. Struijker Boudier (voorzitter)

Prof. Dr. M.J.A.P. Daemen

Prof. Dr. G. Gabbiani (CMU - Genève)

Prof. Dr. M.J. Post

Dr. C.J.M. de Vries (AMC - Amsterdam)

Financial support by the Netherlands Heart Foundation for the publication of this thesis is gratefully acknowledged. The studies described in this thesis were supported by grants of the Netherlands Heart Foundation (NHF-D97.014/D97.167). Printing of this thesis was also financially supported by the Dr. Ir. van de Laar Stichting. 


\section{Contents}

\section{Chapter 1}

General introduction

Regulation and function of smooth muscle cell phenotypic heterogeneity

\section{Chapter 2}

Cultured porcine coronary artery smooth muscle cells: a new model with advanced differentiation

Chapter 3

Smoothelin expression during chicken embryogenesis: detection of an embryonic isoform

\section{Chapter 4}

Expression of the smoothelin gene is mediated by alternative promoters

\section{Chapter 5}

Role of serum response factor and myocardin in transcriptional regulation of smoothelins

\section{Chapter 6}

Structure and chromosome location of Smtn, the mouse smoothelin gene

\section{Chapter 7}

Smoothelin deficiency leads to lethal smooth muscle cell dysfunction in mice

\section{Chapter 8}

General discussion

Smoothelin: from a marker protein to a marked mouse

\section{Summary}

Samenvatting

Bedankt!

Curriculum vitae / List of publications

References 


\section{1}

General introduction

Regulation and function of smooth muscle cell phenotypic heterogeneity 


\section{Introduction}

Smooth muscle cells (SMCs) are essential for a good performance of hollow organs like the vasculature, the lungs, the gastro-intestinal tract, and the urogenital system. The characteristics of the SMCs enable these organs to maintain an adequate functional state by altering their luminal diameters to establish the appropriate physiological blood pressure, peristalsis, or excretion. The ability of the SMCs to contract is critical for this function. However, SMCs are versatile cells in the sense that although they fulfil this defined contractile function, they also retain the capability to perform other functions. They are able to synthesize large amounts of different components of the extracellular matrix (ECM), and migrate and proliferate to a greater extent when stimulated by altered physiological situations or injury. Because of these properties, SMCs are fit to not only regulate the diameters of the lumen of these organs acutely by contraction and relaxation, but also chronically, via structural remodeling by altering cell number and connective tissue composition.

The contractile and synthetic properties of the SMC, collectively referred to as the SMC phenotype, reflect its differentiation state. The mechanisms by which SMC differentiation is regulated are not well understood, despite many studies. The application of techniques that have been successful in unravelling the factors and pathways that lead to differentiation of other cell types does not seem to be suitable for the SMC. This is partly related to the diverse origins of SMCs and the fact that these origins are not always well defined. More importantly, their remarkable phenotypic plasticity as opposed to the terminal differentiation of many other cell types makes it hard to define eventual endpoints of SMC differentiation. The plasticity of SMCs was first fully appreciated by ChamleyCampbell and co-workers ${ }^{4}$, who summarized in vitro evidence indicating that SMC properties rapidly change when growth conditions are altered. Thus, contractile SMCs can convert to synthetic SMCs and vice versa, according to the circumstances. This so-called 'phenotypic modulation' of the SMC limits the application of in vitro studies, and makes it hard to compare results. However, the variability in SMC phenotype in vitro is only a reflection of the variability encountered in vivo. Indeed, marked heterogeneity with respect to morphology, expression of specific SMC marker proteins, proliferation, and migration properties has been observed between SMCs in vivo, both in different tissues as well as within the same tissue. Hence, the variety of SMC functions is matched by the diversity of SMC phenotypes.

The great diversity of SMCs indicates that the paradigm of master transcription factors inducing a uniform differentiation program may not be applicable to this cell type. Nevertheless, it is likely that a basal differentiation program leading to the development of a 'naive SMC' exists. While it is impossible to define 'the SMC', the many different SMCS with slightly different properties share enough 
characteristics to be recognized as SMCs. After basal differentiation, further differentiation results in a fine-tuning of the phenotype and consequently the function of the naive SMC according to the demand of the local environment. This fine-tuning is likely regulated by local factors, although there is also evidence for intrinsic phenotype specific factors. Despite considerable progress in recent years, only the broad outlines of the regulation and function of SMC differentiation and phenotypic modulation have been sketched.

This introduction aims to summarize our current understanding of the regulation of SMC differentiation and phenotypic modulation, and how this is connected to the functional plasticity and the heterogeneity of this cell type. Issues that will be addressed include the SMC phenotypes that have been recognized according to SMC morphology, the various origins of SMCs, the use of marker proteins to characterize SMC phenotypes and heterogeneity, as well as their application in studies on SMC development and SMC differentiation pathways. Also, the in vitro and in vivo model systems that have been developed to this end, and several factors that have an impact on SMC phenotype will be reviewed. In addition, reports on components of the SMC transcriptional program are summarized and a model of SMC phenotype and differentiation regulation is presented. In the last part of this introduction, important aspects of both contractile and synthetic SMC functions will be discussed to appreciate the plasticity of this versatile cell type. Finally, the contractile SMC marker smoothelin, which is the focus of this thesis, will be introduced. 


\section{Smooth muscle cell diversity}

\section{Structure and composition of smooth muscle tissue}

The organization of SMCs differs per tissue. In the vascular system, circularly orientated SMCs are found in the tunica media of arteries and veins, but not in the tunica intima. Usually, this medial layer also contains elastic connective tissue. Larger elastic arteries like the aorta and pulmonary trunk contain many elastic fibers and few SMCs. Muscular arteries like the mesenteric and femoral arteries, in contrast, contain more SMCs and less elastin. In general, veins contain less SMCs in their tunica media, but the larger veins also have longitudinally arranged SMCs in the adventitia. SMCs of visceral organs like the intestines are organized in an inner layer with a circular, and an outer layer with a longitudinal orientation. Furthermore, visceral smooth muscle tissue usually contains fibroblasts that are distributed throughout the muscle. In contrast, the media of blood vessels is homogeneous, containing only SMCs.

Whereas SMCs may be differently organized among organs, they are similar regarding their capability of generating a contraction and producing ECM that can transduce this contraction to other parts of the tissue. The cell to ECM ratio however is variable between the different organs and within an organ depending on the conditions. Moreover, the composition of the ECM varies extensively, which in turn has profound consequences for the phenotype and function of SMCs, as will be discussed later.

\section{Smooth muscle cell characteristics}

\section{Ultrastructure of SMCS}

Changes in SMC phenotype become rapidly manifest in its morphology. Hence, to understand the changes in phenotype, it is necessary to first describe the appearance of contractile and synthetic SMCs (figure 1.1).

Contractile SMCs in vivo are elongated, spindle-shaped cells with a diameter of 5$10 \mu \mathrm{m}$ and lengths between 20 and $500 \mu \mathrm{m}$, depending upon their location. They have one centrally located oval nucleus containing two or more nucleoli. The nucleus of synthetic SMCs is larger and contains more nucleoli ${ }^{4}$. In strongly contracted SMCs, the nucleus can be folded. In contractile SMCs, mitochondria are found both at the periphery and the perinuclear region. Their number varies according to the muscle type. Organelles involved in protein synthesis like Golgi complexes, ribosomes, and the rough endoplasmic reticulum are present in small amounts at the nuclear poles of the contractile SMC. In synthetic SMCs, which are broader and less elongated than contractile SMCs, these organelles are present throughout the myoplasm. The sarcoplasmic reticulum, which is poorly developed 


\begin{tabular}{|ll|}
\hline G & Golgi complex \\
M & Mitochondria \\
ER & Endoplasmic reticulum \\
ECM & Extracellular matrix \\
N & Nucleus \\
DB & Dense body \\
DP & Dense plaque \\
C & Caveolae \\
CF & Contractile filaments \\
\hline
\end{tabular}

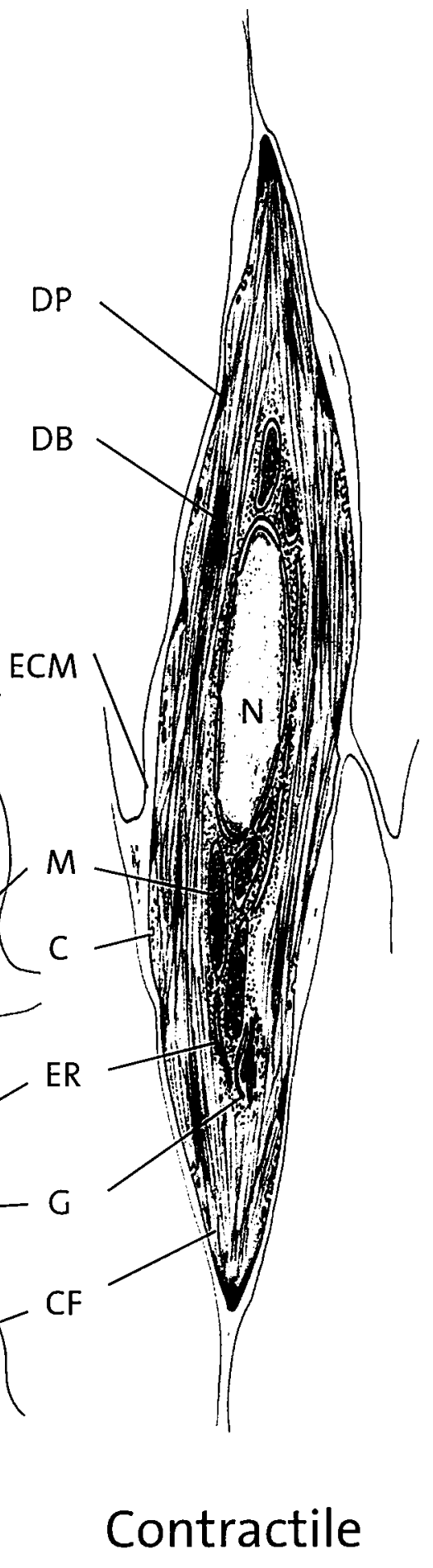

Figure 1.1 Ultrastructural characteristics of contractile and synthetic SMCs. Drawing by G. van Eys. 
in SMCs of either phenotype, is found near the periphery of the cell, continuous with its rough endoplasmic reticulum. The sarcolemma of contractile SMCs has many flask-shaped invaginations which are called caveolae. These structures, which are much less numerous in synthetic SMCs, are often associated with the sarcoplasmic reticulum ${ }^{5}$.

The ECM that embeds synthetic SMCs is much more extensive than the ECM surrounding contractile SMCs. Both types of $S M C$ are surrounded by a marked basal lamina, which varies in thickness in different organs. It is sometimes interrupted by gap junctions, which allow excitatory impulses to pass directly from one SMC to another. In addition, the SMC membrane has adherens type junctions called dense plaques, which are involved in cell adhesion and hence less abundant in synthetic SMCs which have an increased migratory capacity. In cultured SMCs, dense plaques are represented by focal adhesions? ${ }^{7}$. Dense plaques provide anchorage sites for the contractile filaments which make up most of the cytoplasm of contractile but not synthetic SMCs. In cultured SMCs, the contractile filaments form stress fibers, which primarily consist of $\alpha$-smooth muscle actin ( $\alpha$-SMA), but also contain minifilaments of myosin ${ }^{8}$. Intracellularly, the contractile filaments attach to so-called dense bodies, which are thought to functionally resemble the Zline of striated muscle. These dense bodies have also been suggested to relay contraction signals to the basal lamina and surrounding SMCs by coupling cytoskeletal and actin filaments ${ }^{8}$. Contractile as well as synthetic SMCs have an extensive intermediate filament network as part of their cytoskeleton, which shows extensive overlap with the actin and myosin contractile filaments ${ }^{10,11}$. These intermediate filaments are thought to have important functions in controlling cell shape and migration, as well as the transduction of pressure and force.

\section{SMC properties in vitro}

Whereas the diverse appearances of SMCs can be accurately studied in vivo, the properties that are associated with the different morphologies require in vitro studies. Most in vitro studies have used rat aortic SMCs as model system. In tissue culture of these vascular SMCs, the two most obvious SMC phenotypes from a morphological point of view are epithelioid and ribbon- or spindle-shaped cells, which represent the synthetic and the contractile phenotype, respectively ${ }^{12}$ (figure 1.2). Like contractile SMCs in vivo, spindle-shaped cells in culture are thin and elongated. They grow in a hills-and-valleys pattern upon reaching confluency. These hills are made up of 10-15 layers of piled up cells. The valleys contain 0-3 cell layers. Epithelioid cells are polygonal and have a cobblestone appearance. In contrast to the spindle-shaped cells, they stop growing when they reach confluency.

In SMCs isolated from human arteries, the same phenotypes have been described. Both types are capable of maintaining their morphology, even when growing in conditioned medium from the other type ${ }^{13}$. Human spindle-shaped cells become senescent around the 40th passage, but epithelioid cells have a much longer lifespan, 

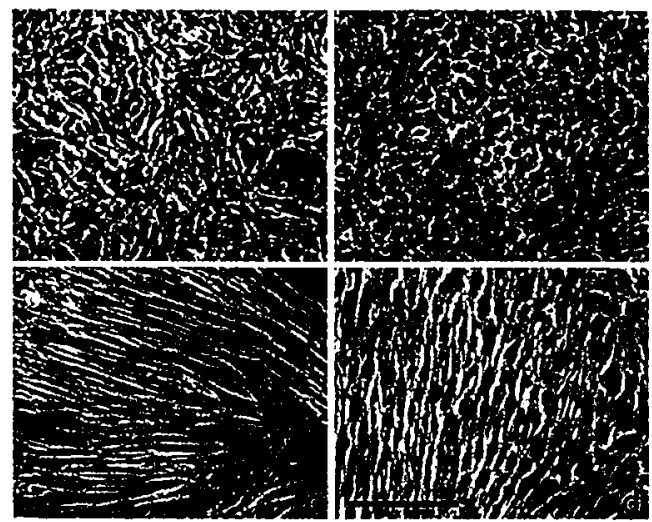

Figure 1.2 The different morphologies of SMCs. Phase-contrast microphotographs of rat SMCs with (a) spindle and (b) epithelioid morphology, and porcine SMCs with (c) spindle and (d) rhomboid morphology. Bar $=150 \mu \mathrm{m}$. Adapted from Hao et al. $(2003)^{559}$.

lasting for at least 60 passages. Epithelioid SMCs of most species exhibit a much higher growth rate and migratory activity than spindle-shaped SMCs.

Apart from epithelioid and spindle-shaped SMCs, so-called rhomboid SMCs have been described ${ }^{3}$. These cells resemble epithelioid SMCs, but are more elongated (figure 1.2). They form a monolayer at confluency and proliferate and migrate more actively than spindle-shaped SMCs.

A less common phenotype, the thin-elongated SMC, has been described in the rat $^{12,14}$. Thin-elongated cells form typical whorls and have a migration activity intermediate between those of spindle-shaped and epithelioid clones. Their proliferation properties resemble those of spindle-shaped cells, and these cells are also thought to represent contractile SMCs.

It will be clear that primary tissue culture systems contain a mixture of SMCs with different phenotypes. In these cultures, the spindle-shaped phenotype is rarely stable. This is probably related to the fact that many primary spindle-shaped SMCS undergo rapid senescent changes in culture, which are characterized by the appearance of large vacuoles. In addition, because epithelioid SMCs proliferate more actively, they outgrow the spindle-shaped cells. It must however be noted that epithelioid cells are also more prone to apoptosis ${ }^{15}$.

\section{SMC phenotypic modulation}

Even though cloned spindle-shaped and thin-elongated SMCs are apt to conversions towards the synthetic phenotype in culture, this usually does not proceed to the point that they morphologically resemble epithelioid SMCs. Conversely, phenotypic modulation of cloned epithelioid SMCs towards SMCs with a thin-elongated or spindle-shaped morphology has never been described. It is possible though to partially regain a contractile phenotype, as indicated by decreased proliferation and migration, and altered protein expression profiles (see below). This occurs exclusively when cultures have either been confluent for a longer time, or grow under specific conditions, i.e. without serum or in the presence of specific compounds that promote maintenance of the contractile 
phenotype ${ }^{16}$. In vivo, modulation from a contractile to a synthetic phenotype and back to a contractile phenotype is frequent, particularly during physiological remodeling (for example in the uterus and uterine blood vessels during pregnanc $y^{17}$ ), or during neointimal formation ${ }^{18,19}$ (see page 71 ). In these cases, the contractile properties of the SMCs are fully restored. Thus, whereas most SMCs in vivo are of the contractile type, stress conditions provoke reversible modulation towards a synthetic phenotype, which also becomes the predominant phenotype during SMC culturing. The phenotype transition is accompanied by changes of morphology, higher proliferation and migration, and expression of different amounts or sets of proteins, as will be described in the next section.

\section{The difference between dedifferentiation and phenotypic modulation}

The terminology with respect to SMC differentiation and phenotypic modulation often leads to confusion. Many researchers use the term 'differentiation' to describe the process by which a SMC acquires a contractile phenotype, and refer to the process by which a SMC modulates to a synthetic phenotype as 'dedifferentiation'. We would like to stress that there is an important distinction between the processes of dedifferentiation and phenotypic modulation. The term 'dedifferentiation' is meant to indicate that a cell returns to a multipotential state. It has however never been shown that SMCs can return to an uncommitted state from where they can differentiate to an entirely different cell type. Thus, we propose that both contractile and synthetic SMCs are differentiated, albeit that they have a distinct function. This view acknowledges the fact that synthetic SMCs are not only associated with disease but are also necessary for physiological remodeling. The term 'phenotypic modulation' should be reserved to indicate shuttling of SMCs between the two ends of the phenotype spectrum (figure 1.3).

\section{Smooth muscle cell marker proteins}

\section{SMC markers and SMC phenotype markers}

Marker proteins usually serve to discriminate between different cell types. Because SMCs can adopt different phenotypes that are associated with a whole range of different protein expression profiles, it is imprudent to identify a SMC by using just one marker protein. Rather, the combined expression of several more or less SMCrestricted markers is required to conclusively distinguish a SMC from other cells that resemble SMCs, like (myo)fibroblasts. SMC marker proteins are also used for the identification of specific phenotypes. On the basis of expression of different markers, attempts have been made to translate the morphological classification of SMCs into a more functional one where epithelioid cells are of the synthetic and spindle-shaped cells of the contractile phenotype. Unfortunately, the relation between SMC morphology and marker expression is not consistent. Nevertheless, it is clear that spindle-shaped cells generally express higher levels of contractile 


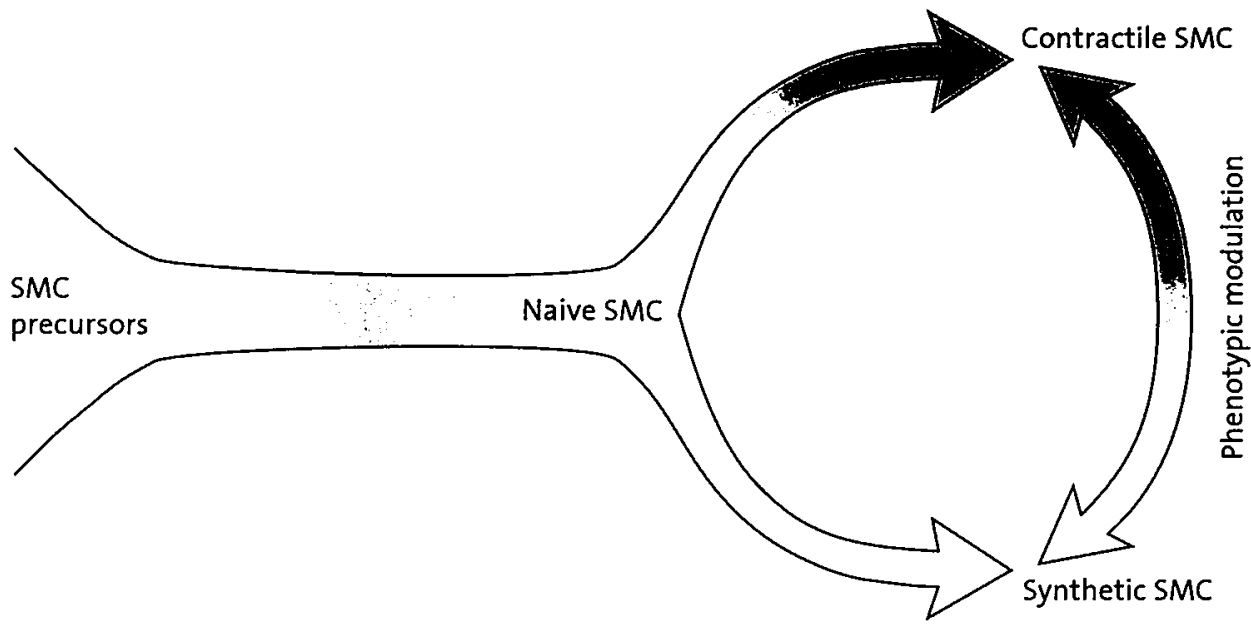

SMC differentiation

Figure 1.3 Schematic representation of SMC differentiation pathways. SMC precursors may differentiate towards SMCs with contractile, synthetic, or intermediate phenotypes. Differentiated SMCs can reversibly change their phenotype, a process called phenotypic modulation. Dedifferentiation of SMCs does not occur. Drawing by $\mathrm{S}$. Ghesquiere.

apparatus-associated proteins, whereas epithelioid SMCs contain less contractile proteins but synthesize more ECM and ECM-degrading proteins.

Most of the SMC marker proteins described to date are involved in contraction of the SMC, either as a structural component of the contractile apparatus itself, or as a regulator of the contraction. Thus, the majority of SMC markers are associated with the contractile phenotype. SMC-specific markers of the synthetic phenotype are scarce. Instead, disappearance of proteins associated with the contractile phenotype is generally taken as a sign of modulation towards the synthetic phenotype. In addition, changes in ratios of specific splice variants of contractile proteins (heavy caldesmon (h-caldesmon), meta-vinculin) have been used to indicate a synthetic phenotype ${ }^{20,21}$, together with induction of matrix degrading enzymes ${ }^{22}$.

In some cases, a particular SMC phenotype has been claimed to be associated with the expression of one particular protein ${ }^{23,24}$. However, it is obvious that only specific repertoires of SMC markers can be used to accurately describe the different SMC phenotypes (figure 1.4). We suggest that at present, the combination of smooth muscle myosin heavy chain (SM-MHC) and smoothelin expression can be used to define the differentiated contractile phenotype. A clear definition of the differentiated synthetic phenotype is much more challenging, because investigators have generally not considered a synthetic SMC as a differentiated $S M C$ but rather as a dedifferentiated SMC, on its way to become a contractile SMC. 


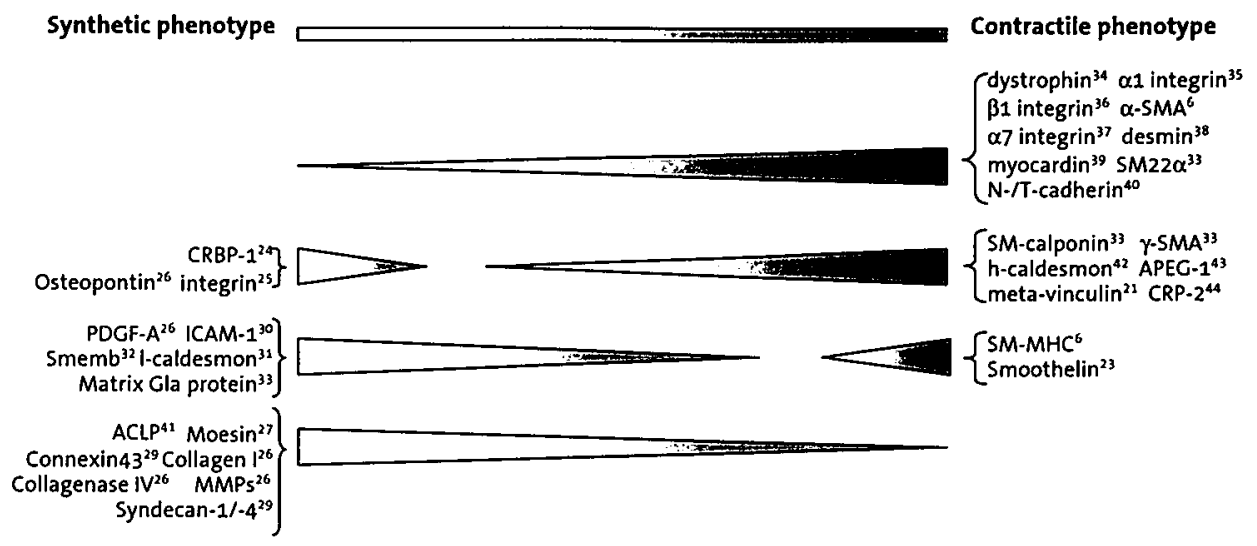

Figure 1.4 Schematic representation of the specificity of gene expression for a particular SMC phenotype. Note that not all of the indicated proteins are SMC-specific. Numbers indicate key references.

Cellular retinol binding protein (CRBP)-1 and osteopontin have been suggested to represent suitable synthetic SMC markers ${ }^{24,26,33}$, and combined expression of these proteins presently appears to be the best indication of a synthetic phenotype. Additional markers of synthetic SMCs will probably have to be deduced from studies addressing changes in phenotype during physiological remodeling or disease.

\section{Characteristics of smooth muscle cell marker proteins}

SMC marker proteins include contractile proteins, contractile filament-associated proteins, and some nuclear or secreted proteins. The SMC marker proteins that will be discussed here have been widely used in the field of SMC differentiation, phenotypic modulation, and function. Some of their characteristics are summarized in table 1.1.

\section{Contractile marker proteins}

$\alpha$-SMA is the most commonly used SMC marker protein. Its popularity is related to the fact that it is the most abundant protein in SMCs, representing $40 \%$ of the total protein content of vascular $S M C s^{45}$. However, $\alpha-S M A$ is not a very specific marker, since it is also found in (myo)fibroblasts, astrocytes, and maybe even endothelial cells $^{46-48}$. The majority of the SMC markers described to date are only specific for SMCs in adults. Likewise, $\alpha$-SMA is present in developing striated muscle cells in embryogenesis ${ }^{49}$. Its expression in SMCs precedes that of all the other SMC marker proteins $^{50}$.

$\gamma$-SMA is a second actin isoform which is SMC-restricted in adults but also detected in developing cardiac muscle. It is more abundant than $\alpha$-SMA in mature gastro- 


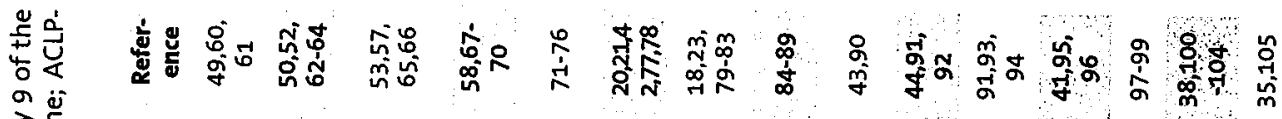

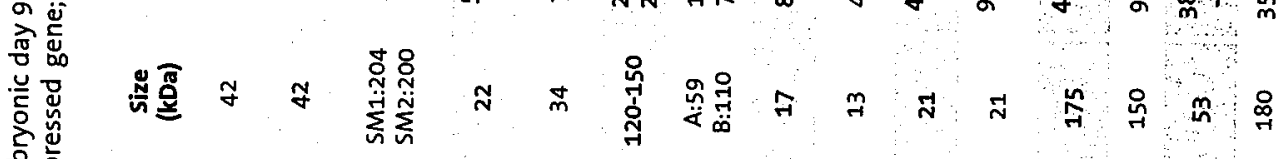
है

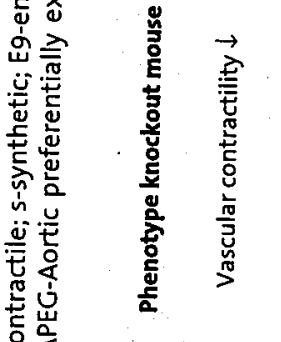

它

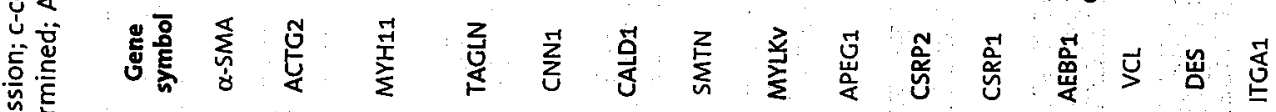
幽要 文苋

苛获 安 它节

乡ู 항웜

ㄴ.

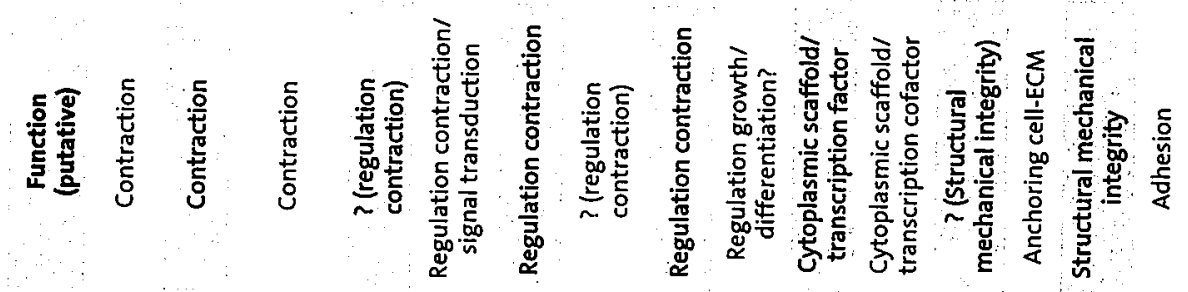
옹

है

نั

它市的

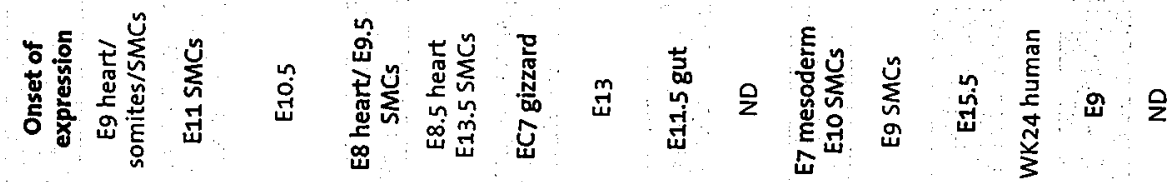

ชั 논

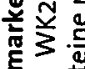
灾荡 解 过 状范 웡 등

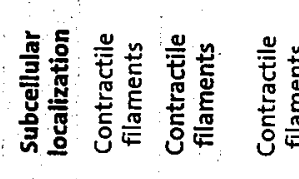

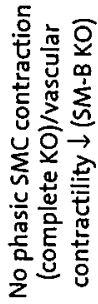

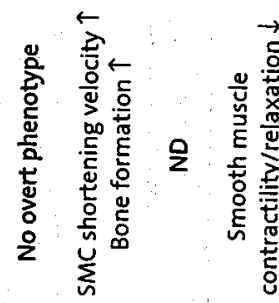

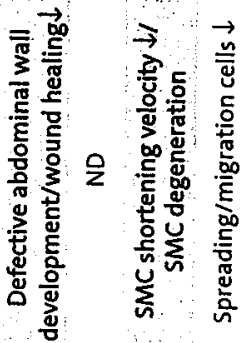
t亩

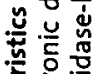

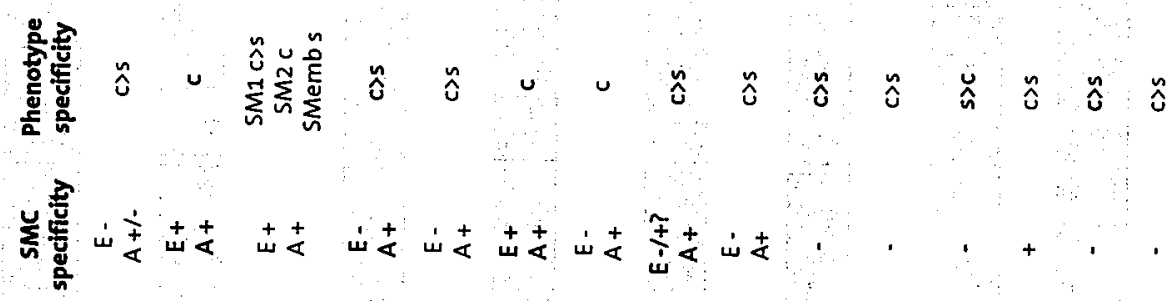
政 裙 究出

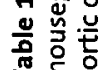

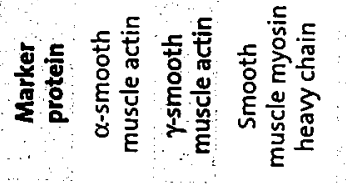

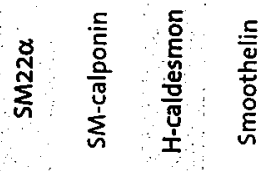

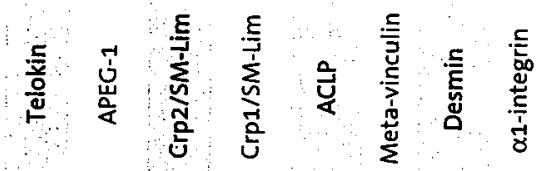


intestinal muscle ${ }^{16}$. Its expression in the vascular system and gastro-intestinal tissues is upregulated during late SMC development ${ }^{51,52}$. Both $\alpha$-SMA and $\gamma$-SMA levels are diminished in synthetic SMCs.

Myosin is the other major contractile protein. In SMCs, myosin consists of two SM$\mathrm{MHCs}$, two $17 \mathrm{kDa}$ myosin light chains $\left(\mathrm{MLC}_{17}\right)$, and two regulatory $20 \mathrm{kDa}$ myosin light chains $\left(M^{2} C_{20}\right)$. The light chains are not used as SMC markers, but SM-MHC isoforms are widely accepted markers of the contractile SMC phenotype. SMCs express four different SMC-specific isoforms of the myosin heavy chain (SM1A, $S M 1 B, S M 2 A$ \& SM2B), all transcribed from the single SM-MHC gene. The SM1 and $S M 2$ variants are generated by alternative splicing of the $3^{\prime}$ end of the gene. SM1 is found in both embryonic and adult SMCs, whereas SM2 is only detected in adult SMCs. Vascular and visceral SMCs have different SM1/SM2 ratios, and even within the vasculature, $S M-1$ and $S M-2$ variants are differentially expressed during development ${ }^{53-55}$. A second alternative splicing in the $5^{\prime}$ region generates SM-A or SM-B isoforms, which are developmentally regulated in a complicated tissuespecific way ${ }^{56}$. In contrast to many other SMC markers, the SM-MHC gene is not expressed in other cell types at any time during development or adulthood ${ }^{57}$.

Besides SM-MHC, SMCs also express two MHC isoforms that are not SMCrestricted, non-muscle MHC-A (NMHC-A) and NMHC-B/SMemb. During phenotypic modulation towards the synthetic phenotype, SM-MHC is quickly and strongly downregulated, and exchanged for the non-muscle MHC isoforms. Specifically $S M e m b$ expression is upregulated after vascular injury and in cultured SMCs ${ }^{32}$.

\section{Contractile filament-associated marker proteins}

To attain the specific characteristics of SMC contraction, SMCs express a unique set of contractile filament-associated proteins, comprising SM22 $\alpha$, smooth muscle calponin (SM-calponin), h-caldesmon, and telokin. Besides the SMC-specific contractile proteins themselves, these proteins are the most widely used SMC markers. $S M 22 \alpha$ is an abundant actin-associated $22 \mathrm{kDa}$ protein which is homologous to $S M$-calponin. Like $\alpha-S M A$, the SM22 $\alpha$ gene is transiently expressed in heart and somites during embryogenesis. After birth, $S M 22 \alpha$ is only expressed in SMCs ${ }^{58}$. SM $22 \alpha$ is downregulated when SMCs modulate towards the synthetic phenotype 33,59 .

Like $\alpha$-SMA and SM22 $\alpha$, SM-calponin is expressed in the developing heart during early embryogenesis, but restricted to SMCs in adults. Unlike these other genes, it is not expressed in the somites of the developing embryo. Also, SM-calponin expression in SMCs starts later than expression of most other SMC markers ${ }^{73}$. Consequently, SM-calponin appears to be a marker for more advanced contractile SMC differentiation.

The SMC-restricted protein h-caldesmon is also associated with the contractile SMC phenotype, since it is downregulated in synthetic SMCs in favor of the widely expressed I-caldesmon isoform $\mathrm{m}^{31,42}$. $\mathrm{H}$ - and I-caldesmon are transcribed from the single caldesmon gene via alternative splicing of exons $3 \mathrm{~b}$ and 4 , which are only 
present in h-caldesmon ${ }^{78,106,107}$. Whereas there are no data on the onset of the expression of caldesmon isoforms in mice, l-caldesmon expression precedes that of $\mathrm{h}$-caldesmon in the developing chicken gizzard ${ }^{77}$.

Telokin is a small SMC-restricted protein which is transcribed from the $3^{\prime}$ part of the MLC kinase gene ${ }^{86}$. Visceral SMCs express higher levels of telokin than vascular SMCs. Reports on its expression pattern are somewhat contradictory. Expression in chicken heart and skeletal muscle, albeit at low levels, has been reported by some authors, whereas others state that telokin is not expressed at all in the other muscle lineages during mouse development ${ }^{85,86,108}$.

One of the latest SMC markers described is smoothelin, which is also associated with the contractile SMC phenotype ${ }^{23}$. There are two smoothelin variants, a $59 \mathrm{kDa}$ A-isoform in visceral SMCs, and a $110 \mathrm{kDa} B$-isoform in vascular SMCs ${ }^{80}$. The function of the smoothelins is unclear, but like other SMC markers, they may play a role in SMC contraction ${ }^{23}$.

\section{Other marker proteins}

There are many other proteins which are developmentally regulated in SMCs or show heterogeneous expression patterns in different SMCs, but their expression is not specific for SMCs. Technically, these proteins cannot be used as SMC markers. However, since they have been used in several important studies on SMC differentiation and phenotypic modulation, they will be briefly reviewed here. These proteins include desmin, meta-vinculin, aortic preferentially expressed gene (APEG-1), Crp/SM-Lim proteins, aortic carboxypeptidase-like protein (ACLP), and different integrins ${ }^{1}$. The best studied protein of this group is the cytoskeletal protein desmin, which levels are quickly downregulated when SMCs are cultured and acquire a synthetic phenotype ${ }^{38}$. Vimentin is the other major intermediate filament protein of SMCs ${ }^{109}$. The desmin/vimentin ratio differs between arteries, with larger elastic arteries displaying a lower ratio than muscular arteries ${ }^{120}$. APEG1 and Crp/SM-Lim proteins localize to the nucleus. Similar to the other SMC markers, they are downregulated when SMCs modulate towards the synthetic phenotype ${ }^{43,44}$. ACLP is a secreted protein that is predominantly expressed in vascular SMCs and found in the ECM ${ }^{41}$. It is upregulated during differentiation of neural crest cells towards SMCs in vitro, but also when SMC acquire a synthetic phenotype.

\section{Temporal progression of SMC marker gene expression}

By looking at the onset of expression of several SMC marker proteins during chicken embryogenesis and assuming that this reflects the degree of contractile SMC differentiation, Owens has composed a SMC differentiation scale 1 . Marker proteins that were included are $\alpha$-SMA, SM-MHC-1 and -2, SM-calponin, SM $22 \alpha$, $1 E 12, S M-\alpha$-tropomyosin, and $h$-caldesmon. Since the expression of most of these proteins has now been studied in the mouse, a SMC differentiation scale of this species, including new SMC marker proteins, is presented in figure 1.5. It must be 


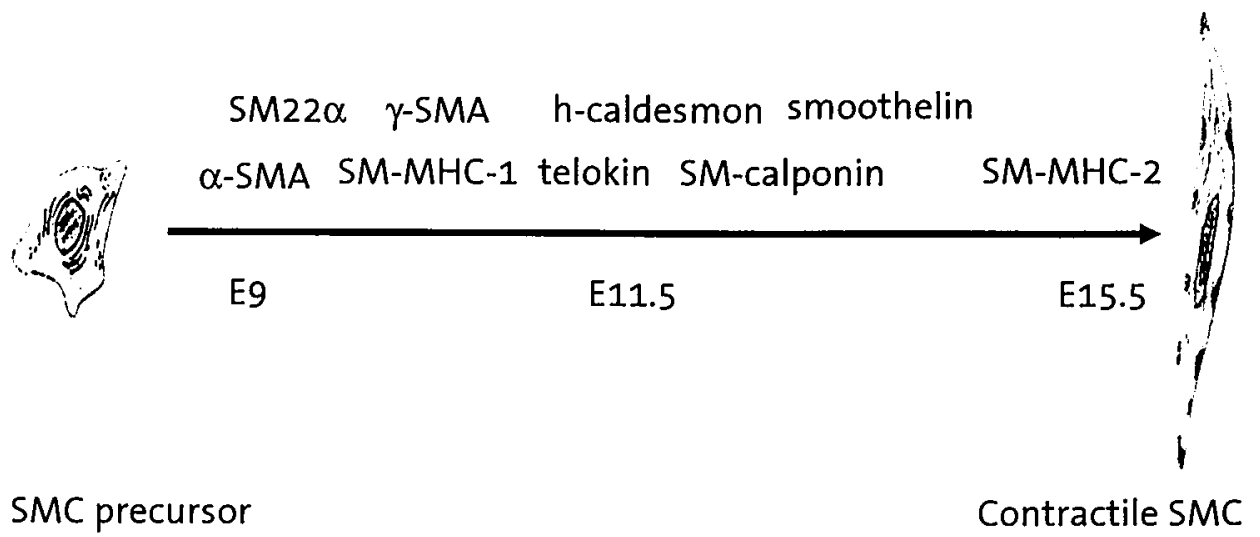

Figure 1.5 Onset of expression of various contractile SMC markers during mouse embryogenesis. Adapted from Owens $(1995)^{1}$.

kept in mind that there are no studies that have outlined the expression pattern of all markers in one model. Moreover, the sensitivity of the detection methods varies greatly between studies. Comparison of the degree of contractile SMC differentiation between different models therefore remains intricate.

The described proteins represent the majority of the markers for the SMC lineage described today. They have been used as tools for identifying cis-elements and transcription factors that direct their SMC-specific expression. Moreover, they have allowed the study of SMC development and differentiation mechanisms, which will be discussed in the next section.

\section{Smooth muscle cell origin, development, and aging: a road towards diversity}

Smooth muscle cell specification mechanisms

Lineage studies distinguish two steps in the process of commitment of a cell to a particular cell type: specification and determination. A specified cell has been defined as a pluripotent cell that is capable to differentiate autonomously in vitro, while a determined cell can differentiate autonomously in vivo ${ }^{111}$. In pluripotent cells, specification and determination programs are activated according to the integration of many signals. Specific transcriptional programs are initiated or silenced, thereby restricting fate choices and creating a specified cell. During differentiation, activation or silencing of other transcriptional programs results in a cell that has a defined and specialized function associated with the cell type and its location. As such, differentiation becomes apparent in the expression of specific proteins that act in concert to shape the cell with respect to morphology, responses to stimuli that are typical for its location, and ultimately physiological function. 
For SMCs, the distinction between specification and determination is irrelevant. SMC precursors that can be induced to differentiate to contractile and synthetic SMCs in vitro have been described, but reports on putative SMC precursors that have been implanted into regions where no SMCs are present have not been published. This is due to the fact that SMCs are present throughout the body, always conferring the appropriate inductive signals for SMC development. Considerable progress has been made in the field of SMC lineage specification in recent years as a result of the use of embryonic stem (ES) cells. ES cells are undifferentiated, pluripotent cells which are derived from the inner cell mass of blastocysts. Under certain conditions, they form aggregates of differentiating cells which are called 'embryoid bodies'. With further culturing, many early embryonic lineages among which cardiac, skeletal, and smooth muscle lineages arise $\mathrm{e}^{112}$. This differentiation process mimics the events that happen during normal embryonic development. Using this system, the development of contracting SMCs out of embryoid bodies and the existence of divergent differentiation pathways between visceral and vascular SMCs has been demonstrated ${ }^{113}$. More recently, an important role for the transcriptional co-activator myocardin in early SMC differentiation was shown using mouse ES cells ${ }^{114}$. In these cells, myocardin activated expression of a number of SMC-specific genes like $\alpha$-SMA, SM22 $\alpha$, and SM-calponin. Moreover, myocardin null mice display a complete lack of vascular SMCs ${ }^{115}$, indicating that myocardin is one of the critical factors specifying this SMC lineage. Using ES cells and embryoid bodies, it has also been demonstrated that endothelial cells, haematopoietic cells, and SMCs have a common descent ${ }^{116,117}$. In these experiments, platelet derived growth factor-B (PDGF-B), vascular endothelial growth factor (VEGF), and the transcription factors fetal liver kinase (Flk)1 and Tcell acute lymphocytic leukemia (Tal)1 were shown to regulate cell fate choice in a combinatorial way.

It is obvious that many additional factors are involved in SMC specification mechanisms. Thus, further development of embryoid body and ES cell techniques is required to delineate the pathways by which the different types of SMCS arise.

\section{Common developmental pathway of muscle lineages}

It is well known that during normal embryonic development, immature striated muscle cells transiently express many SMC isoforms of contractile proteins, which are later replaced by the skeletal isoforms. The functional significance of this phenomenon is unclear. However, it may be related to the hypoxic conditions that are present at this embryonic stage and the fact that the SMC isoforms need less energy to perform adequately. Cardiomyocytes re-express many SMC isoforms of contractile proteins under hypoxic conditions, supporting this idea ${ }^{118}$. Mechanisms that are responsible for transient SMC isoform expression in striated muscle are largely unknown, though several of the skeletal and cardiac muscle specific promoters have been shown to contain functional motifs that are also important for regulation of SMC-specific expression ${ }^{119.120}$. Thus, activation of (a part of) the 
SMC differentiation program appears to be responsible for the SMC-like phenotype of developing striated muscle cells. However, the induction of striated musclespecific transcription factors directs further development towards a terminally differentiated striated muscle cell, and apparently overrules SMC-specific transcriptional programs.

\section{Smooth muscle cell origins}

The onset of $\alpha$-SMA expression is generally taken as the first sign of SMC development. Using this starting point, it is clear that the majority of SMCs arises from the mesoderm ${ }^{121}$. However, SMCs also originate from the neural crest and from transdifferentiation of various other cell types.

During development of most smooth muscle tissues, mesodermal precursor SMCs associate with the already formed epithelium or endothelium, and subsequently start to express marker proteins of smooth muscle differentiation. This interaction is mediated by the expression of specific integrins and adhesion molecules, which are only detected in developing SMCs ${ }^{122}$. In the vasculature, newly recruited precursor SMCs in additional layers express these markers in a ventral to dorsal pattern ${ }^{123}$. The mechanisms directing the association of SMC precursors and epithelial/endothelial cells are largely unknown, but are likely to be driven by local environmental factors.

SMCs in the upper parts of the great vessels and the cranial vasculature are of neural crest origin ${ }^{124}$. These SMCs contribute to the elastic properties of these vessels by initiating elastogenesis ${ }^{125}$. Although there are regions where no morphological differences are visible between SMCs of neural crest- and nonneural crest-origin (e.g. the aortic and pulmonary trunk roots), the origin boundaries are usually well defined ${ }^{45}$. These differences in origin become manifested when mesodermal SMCs are made to replace SMCS of neural crest origin. In this case, the organization of the elastic fibers around the SMCs turns out to be disordered and marker protein levels change ${ }^{126}$. Furthermore, mesodermally derived aortic SMCs have distinct contractile characteristics and growth patterns compared to neural crest-derived aortic SMCs ${ }^{127-129}$.

Coronary SMCs stand apart from SMCs of other vessels because they develop exclusively by transdifferentiation of epithelial cells of the pro-epicardial organ ${ }^{130}$. This process is dependent on serum response factor $(\mathrm{SRF})^{51,131}$, a transcription factor which is involved in many aspects of SMC differentiation. Transdifferentiation of embryonic endothelial cells towards SMCs in the aorta has also been reported ${ }^{132}$. SMCs that were shown to arise in this way expressed $\alpha$-SMA, SM22 $\alpha, S M$-calponin, and $h$-caldesmon (Dr. M. de Ruiter, personal communication). Interestingly, there is accumulating evidence that endothelial cells and SMCs can arise from a common precursor in adults (see page 24). 


\section{Vascular smooth muscle cell development in vivo}

Before $\alpha$-SMA expression can be detected, developing vascular SMCs can be identified on the basis of anatomical location within the vessel wall ${ }^{45}$. They are described as fibroblast-like cells which lack a basement membrane but do have well developed protein synthesis organelles. These SMC precursors have a euchromatic nucleus and only few filaments in their cytoplasm. During further development to SMCs, they acquire a heterochromatic nucleus and build up large amounts of myofilaments in their cytoplasm, while concomitantly losing synthetic organelles ${ }^{133.134}$. The thin actin filaments appear earlier than the thick myosin filaments, which appear at the same time as the basal lamina ${ }^{4}$. In the mouse embryo, vascular SMCs can first be identified by morphology in the dorsal aorta at embryonic day (E) $9.5^{50}$. These SMCs proliferate actively and express low levels of SMC-specific marker proteins, such as SM-MHC, SM22 $\alpha$, and SM-calponin ${ }^{57.69 .73}$. In human aortic SMCs, a similar pattern of development is observed, with initially low expression of $\alpha$-SMA, SM-MHC, h-caldesmon, and meta-vinculin ${ }^{99}$.

After birth, SMC proliferation slows down abruptly, but vessel wall growth still occurs via hypertrophy. Stabilization of the vessel wall is achieved via high production of ECM proteins. For example, the volume of the ECM of the rat aorta increases tenfold in the first two weeks after birth ${ }^{135}$. The myofilaments, then comprising $\sim 8 \%$ of the cell volume, are confined to the periphery of the cell, while the synthetic organelles $(\sim 30 \%)$ are present throughout the cell. At this stage, increasing expression of contractile proteins like SM-calponin and $S M 22 \alpha$, but also of ECM proteins like elastin and collagen is seen ${ }^{136}$. In rats, contractile and ECM gene expression is normalized to basal adult levels two to three months after birth. The amount of elastin and collagen does not increase anymore and there is myofilament build-up until the adult level of $70 \%$ of the cell volume is reached. Synthetic organelles make up $10 \%$ of the cell volume in adult SMCs ${ }^{4}$. Human aortic SMCs do not express adult levels of h-caldesmon and meta-vinculin even six months after birth, indicating that maturation of SMCs as monitored by these markers of advanced contractile differentiation proceeds slowly ${ }^{99}$. Only by the age of 18 months, expression of $\alpha-S M A, S M-M H C$, meta-vinculin, and h-caldesmon resembling the adult levels was seen ${ }^{97}$.

\section{Visceral smooth muscle cell development in vivo}

Much of the work on the development of visceral SMCs has been done in the chicken. Like vascular SMCs, visceral SMCs can first be identified by morphology when precursor mesenchymal cells elongate and align in a relatively dense cellular layer. Subsequently, they start to express $\alpha$-SMA, SM-MHC, and $\gamma-S M A^{50}$. In contrast to some populations of vascular SMCS which arise by migration of precursors, differentiation of precursor cells to visceral SMCs seems to be a local process which does not depend on migration. At birth, visceral organs are developed to a different extent, e.g. intestinal SMCs are more differentiated than SMCs of the vas deferens ${ }^{137}$. 
According to morphology, differentiation of visceral SMCs does not appear to begin at one point followed by spreading, but rather occurs simultaneously along the entire length of a particular organ ${ }^{137}$. $\alpha$-SMA expression, in contrast, does not start simultaneously, but shows cranial to caudal gradients. This exemplifies the frequent discrepancy between SMC morphology and marker gene expression. At E8.5, the first visceral SMCs in the chicken ileum start to express $\alpha$-SMA. At this stage, the cells are still rounded, only becoming elongated at E10.5. They contain thin actin filament bundles with a small dense body halfway along the length of the bundle ${ }^{137}$. These actin filaments do not attach to dense plaques at the membrane until after birth. Advanced differentiation of the chicken gizzard as defined by SM-MHC and SM-calponin expression starts in the outer part of a thick layer of undifferentiated mesenchymal cells. Subsequently, the outer already differentiated SMCs induce differentiation of the inner layers into SMCs ${ }^{138}$. Differentiation of the circular layer of visceral smooth muscle precedes differentiation of the longitudinal layer. Concomitant with the onset of contractile gene expression, ECM protein expression commences. At E14, mouse bladder SMCs express elastin, various collagen types, laminin, and fibronectin ${ }^{139}$. Levels of these proteins increase during development, with the exception of fibronectin and collagen type IV levels, which decline. After birth, visceral SMCs greatly increase their cell volume, mainly because of an increased length ${ }^{137}$. The temporal progression of visceral and vascular SMC differentiation differs to some extent. Differentiation of visceral SMCS is completed soon after birth, in contrast to vascular SMC differentiation, which takes much longer. This is thought to be related to the dramatic hemodynamic changes that occur after birth, leading to deposition of ECM proteins. Why the new conditions after birth do not lead to similar changes in the phenotype of visceral SMCs is unclear.

\section{Transdifferentiation}

There is accumulating evidence that under certain in vitro conditions, several adult cell types are able to transdifferentiate towards SMCs. Specifically bone marrow cells and endothelial cells have been implicated. For example, a large proportion of cultured human umbilical vein endothelium-derived cells (HUVECs) differentiate towards cells resembling contractile SMCs upon deprivation of fibroblast growth factor $(F G F)^{140}$. Bovine aortic endothelial cells have also been shown to be able to acquire a contractile SMC phenotype via transforming growth factor (TGF)- $\beta_{1}$ dependent and cell-cell contact-dependent transdifferentiation ${ }^{141}$. Bone marrow stromal cells expressing platelet derived growth factor (PDGF)-B can directly differentiate into SMCs with a contractile phenotype as well, but the mechanism involved seems to be distinct from endothelium-derived $S M C s^{142}$. In addition, circulating human mononuclear cells cultured in PDGF-B enriched medium have been shown to adopt SMC characteristics with respect to morphology, marker protein profile, and growth in a hills-and-valleys pattern ${ }^{143}$. Nevertheless, they can be distinguished from regular SMCs because they express the surface marker CD34, 
which is normally absent in SMCs. Indeed, cells at an intermediate point of development that co-expressed both endothelial and SMC markers are consistently detected in this type of studies. The phenomenon of transdifferentiation is rare, since the frequency in primary endothelial cell cultures was.only 0.01-0.03\%. In addition, it is unknown to what extent these in vitro findings can be extrapolated to the situation in vivo.

\section{Smooth muscle cell aging}

From studies on animal models of atherosclerosis, it is known that SMC properties change with age ${ }^{144}$. They acquire a higher proliferation index and express more ECM proteins, indicating a synthetic phenotype. Because it is difficult to study SMC aging in a coordinate way in vivo, most of the studies addressing this topic have employed cultured SMCs. In these studies, effects of aging are evident. In general, SMCs cultured from young animals are better able to maintain a contractile phenotype than SMCs from older animals.

Rat embryonic SMCs that do not have a contractile phenotype yet are capable of mitogen-independent growth, a characteristic that is lost after E2O. It has been shown that growth of these embryonic SMCs is driven by a mechanism distinct from mitogens that are known to affect adult SMC proliferation ${ }^{145}$. Genes that could be involved in regulating this autonomous growth potential have been identified by a subtractive hybridization approach, but have not been elaborated on ${ }^{146}$. Similar to embryonic SMCs, SMCs cultured from fetal rats have a great proliferative potential. However, these cells also keep the contractile phenotype that they have already acquired. Likewise, SMCs isolated from neonatal rats maintain high expression of $\alpha$-SMA, $\gamma$-SMA, SM-calponin, and SM-MHC in culture ${ }^{12,136}$. In an independent study, SMCs from newborn rats kept the level of $\alpha$ SMA high, but showed a modest decrease of desmin and SM-MHC when cultured ${ }^{38}$. Cultured adult SMCs gradually lose $\alpha$-SMA, SM-MHC, and desmin expression, indicative of increased modulation towards the synthetic phenotype. SMCs cultured from old rats (>18 months) express even lower amounts of $\alpha$-SMA and $S M-M H C$, and proliferate more actively ${ }^{147}$. The loss of contractile gene expression with increasing age appears to translate to a diminished contractile performance of the $S M C^{148}$. Besides the diminished contractile protein levels at higher age, synthesis of ECM proteins in cultured SMCs also decreases with an increased age of the donor ${ }^{4,149}$. In addition, ECM reorganization occurs, which is associated with fragmentation of the elastic fibers ${ }^{150}$. Most of the described effects of age on SMC phenotype have been studied using rat aortic SMCs. However, the same pattern has been observed in SMCs of the bovine pulmonary artery and the rabbit aorta ${ }^{98,152}$.

So far, mechanisms responsible for the observed changes in protein levels are largely unknown, although it has been shown that SM-MHC levels at different ages are regulated at the transcriptional level ${ }^{152}$. 
Future studies on this topic may take advantage of micro array techniques and proteomic approaches, which allow reliable identification of differentially expressed genes and proteins between SMCs derived from old versus young animals. Moreover, signalling pathways that are responsible for the altered phenotype can be uncovered in this way.

\section{Smooth muscle cell heterogeneity in vivo and in vitro}

It will be clear from the previous sections that SMCs are heterogeneous with respect to morphology, migratory activity, and proliferative potential. At the molecular level, these differences translate into differential expression of contractile machinery proteins, but also cytoskeletal and ECM proteins. SMC heterogeneity may be partly caused by diverse origins and different local environments. However, since the variability is also encountered between SMCs within tissues, heterogeneity appears to be an intrinsic property of SMCs with important functional consequences. Because of the diversity, the many different SMCs in a particular tissue are able to fulfil distinct functions. Together with the ability of SMCs to acquire different properties by the process of phenotypic modulation, SMC heterogeneity provides smooth muscle tissues with a unique flexibility to respond to changing conditions. Below, some important studies that have contributed to our understanding of the occurrence of different types of SMCS in the vascular wall are reviewed, and the functional importance of SMC heterogeneity will be outlined.

\section{Smooth muscle cell heterogeneity between tissues}

Even though they are readily identified as SMCs, it is obvious that SMCs of different tissues differ profoundly. In many cases, this is probably a result of the distinct environment. However, even in similar tissues that are in close proximity, markedly different SMC properties have been encountered. For example, SMCs of the closely spaced pig coronary and internal mammary arteries have a clearly different gene expression profile, as revealed by a suppression subtractive hybridization approach $^{153}$. Tight and intermediate junction genes had higher expression levels in the mammary artery, whereas inflammation/proliferation-associated gene products were more abundant in the coronary artery. Since these arteries arise from distinct precursors, the observed differences may still be explained by their distinct origin.

Heterogeneity between SMCs of the same vascular bed (i.e. human coronary arteries) has however also been demonstrated. Larger arteries contained more desmin- and SM-calponin-positive SMCs than smaller arteries, but did not differ regarding vimentin or $h$-caldesmon expression ${ }^{154}$. 


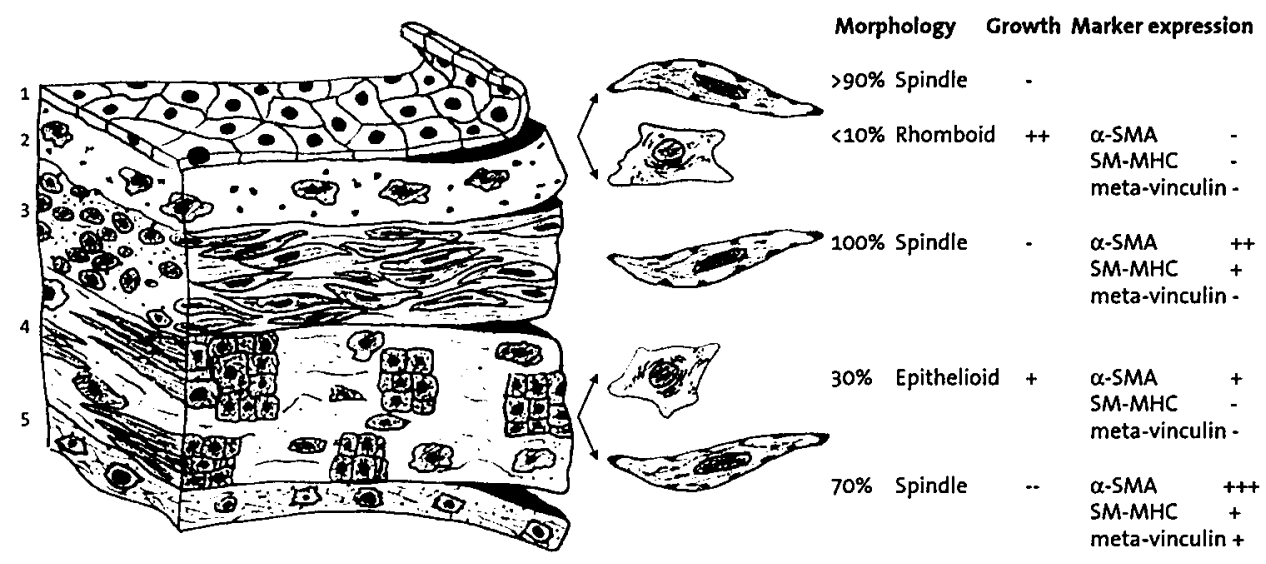

Figure 1.6. Structure of the wall of the bovine aorta and characteristics of SMC types cultured from the different layers. 1-endothelium; 2-subendothelial layer; 3-inner medial layer; 4-outer medial layer; 5adventitia. Based on Frid et al. (1997) ${ }^{155}$.

Smooth muscle cell heterogeneity within tissues

SMC heterogeneity within a tissue was investigated in a systematic way by Frid and colleagues ${ }^{98}$. They showed that in the bovine pulmonary artery, SMCs can be categorized into four different phenotypes, with different marker protein expression profiles and different morphology. The media of this artery was shown to be organized in a sub-endothelial, middle, and outer layer with different mechanical properties due to differences in patterns of cell arrangement and elastin distribution (figure 1.6). SMC characteristics between these layers differed, but also within the outer layer, SMC heterogeneity was apparent. In this layer, cells forming compact clusters which consisted of longitudinally and circumferentially orientated cells could be identified. Interestingly, the observed phenotypes in vivo could also be captured in vitro ${ }^{155}$.

The existence of similar distinct SMC populations within the same tissue has also been demonstrated in the arterial wall of the rat ${ }^{12,156}$, the porcine coronary artery ${ }^{3}$, and the human internal thoracic artery ${ }^{13}$. In the latter model, the heterogeneity was revealed after serial enzymatic digestions and quantification of the cell area of sequentially released SMCs. The cells that were liberated first were significantly smaller than the cells that were obtained from the deeper medial segment. The two cell types were cloned and shown to have an epithelioid or spindle-shaped morphology. They both had a contractile gene expression profile, but only spindleshaped cells expressed meta-vinculin. In addition, they had higher SM-MHC and SM-calponin levels, and a higher h-caldesmon/l-caldesmon ratio. The spindleshaped cells also expressed more type I collagen, showing that synthetic and contractile functions of SMCs are not mutually exclusive. Apart from these differences in SMC marker protein expression, the two types of clones also differed 
with respect to proliferation rate, ECM building, and responses to various growth factors and hormones. One typical spindle-shaped clone derived from the human internal thoracic artery, designated HITB5, was investigated in more detail ${ }^{157}$. Interestingly, this clone adopted either a synthetic or a contractile phenotype, depending on serum concentrations. It represents one of the two described SMC clones that can reversibly modulate to both ends of the phenotype spectrum. The other clone was described in a recent report on cultured porcine coronary arteryderived SMCs. In this study, the method of isolation of the SMCs was shown to influence their characteristics in culture. Enzymatic digestion gave rise to spindleshaped SMCs, whereas explantation yielded primarily rhomboid SMCs ${ }^{3}$. Like in other models, these rhomboid SMCs migrated far more rapidly than spindleshaped SMCs and expressed less contractile proteins. Similar to the HITB5 cells, clones obtained from these primary porcine SMC cultures were capable of reversible phenotypic modulation after FGF-2 or PDGF-B treatment. Since this phenomenon has never been described in the extensively studied rat $S M C$ clones, it appears that human and porcine SMCs are more plastic than the corresponding rodent SMCs.

Not only contractile SMC marker proteins reflect the heterogeneity of SMCs in the arterial wall. Gap junctional proteins and adhesion molecules are also differentially expressed. For example, a complex differential expression of integrin subunits in different SMC subsets in the vessel wall has been described by several groups ${ }^{158-161}$. $\mathrm{N}$-cadherin and T-cadherin are also differentially expressed in aortic SMC layers, with higher levels in SMCs adjacent to endothelial cells ${ }^{162,163}$. In addition, SMCs of elastic arteries have been found to express connexin43, in contrast to SMCs of muscular arteries. A study of the human internal mammary artery, which has both elastic and muscular segments, showed that the levels of connexin 43 and desmin in SMCs in these different parts were inversely correlated ${ }^{28}$. Interestingly, expression of other SMC differentiation markers like $\alpha$-SMA, h-caldesmon, SMcalponin, and SM-MHC did not differ between the two regions, indicating that both SMC populations have a contractile phenotype. SMCs in the great vessels with high levels of connexin 43 are contributed by the neural crest. Thus, it remains to be investigated whether the differential expression of connexin43 in the internal mammary artery is related to SMCs originating from different germ layers.

Although this section focuses on heterogeneity of SMCs in the vascular wall, it should be noted that phenotypic heterogeneity within tissues has also been found in non-vascular SMCs like cultured airway SMCs and rabbit bladder SMCs ${ }^{164,165}$.

\section{Determinants of smooth muscle cell heterogeneity}

There is still a lot of debate about the relative contribution of the local environment versus innate SMC characteristics to SMC heterogeneity. The responsiveness to different serum concentrations of some SMC clones in culture indicates that even if there is innate SMC diversity in the vessel wall, the local environment still has a 
large influence on SMC phenotype. On the other hand, there are also indications that SMC heterogeneity is an intrinsic property of the cell and not a function of the local environment. This notion is supported by a large body of in vitro data, showing that it is feasible to culture clonal SMCs that keep their own phenotype under profoundly different culture conditions. Recently, an elegant study in which cultured arterial SMCs with different phenotypes were implanted in rat carotid arteries also supported this concept $\mathrm{t}^{147}$. The implanted cells, either spindle-shaped cells from newborn rats or epithelioid cells derived from old rats, retained their specific expression pattern of $\alpha$-SMA and SM-MHC in vivo. Moreover, expression of CRBP-1 in epithelioid SMCs was kept for at least 20 days. A longer follow-up was not performed, so it is not known if the properties of the seeded cells change later in the remodeling process. In addition, culturing of the cells may lock them in one particular phenotype through epigenetic mechanisms that act during replication. Yet, this study provides the first in vivo evidence that heterogeneity is an intrinsic quality of SMCs.

The above implies that there are stable transcriptional programs in SMCs which direct expression of genes associated with particular phenotypes. There are many putative regulators of SMC phenotype and consequently SMC heterogeneity, of which SRF is best studied. The concentration gradients and alternatively spliced isoforms that have been described of this factor have specific effects on SMC gene transcription and consequently may be partially responsible for SMC heterogeneity. For example, SRF $\Delta 5$, which lacks exon 5 , has different expression levels along the aorta, with higher expression in the aortic arch and lower levels in the abdominal aorta. This expression pattern is inversely correlated to SM22 $\alpha$ and SM-MHC level ${ }^{166}$. Whether such differential expression is also observed in other arteries is unknown. Different isoforms of SRF are unlikely to be the only determinants of SMC heterogeneity. Other players in the field may include activin $A$ and myocardin, which have a different impact on SMCs of different tissues and a heterogeneous expression pattern in SMCs of different tissues respectively ${ }^{167,168}$. However, while it may be possible to correlate expression levels of transcriptional regulators with the occurrence of different SMCs in different (parts of) tissues, it is clear that the observed heterogeneity between closely spaced SMCs within a tissue cannot yet be explained by differential expression of particular phenotype regulators.

The summarized studies indicate that every tissue has a different SMC composition, depending on its function and the local conditions. We think that these phenotypically different subpopulations of SMCs in the different organs are specialized to fulfil a particular function as efficient as possible. This does not exclude the possibility of a change of functional properties after injury or in a new physiological environment. Nevertheless, it appears that some SMCs maintain their normal contractile function after pathological stimuli, whereas others that are more primed for repair exhibit increased proliferation, migration, and ECM 
synthesis in response to the same stimuli. This kind of response occurs for example after vascular injury in vivo ${ }^{18}$. Although the overall expression level of contractile SMC differentiation markers in this example goes down, immunohistochemical stainings indicate that the response is remarkably heterogeneous between different SMCs in the vessel wall, with some SMCs responding dramatically and others not at all.

The different properties and functions of SMCs are mainly brought about by innate transcriptional programs but can be modulated by the local environment. Importantly, SMC heterogeneity does not compromise the performance of the tissue but rather gives the organ the flexibility that is needed to perform in different physiological or pathological situations. Thus, SMC heterogeneity is an essential property for the correct functioning of smooth muscle tissues. 


\section{Regulation of smooth muscle cell differentiation and phenotypic modulation}

\section{Factors involved in smooth muscle cell development, differentiation and phenotypic modulation}

A better understanding of the molecular mechanisms underlying SMC differentiation and phenotypic modulation will help us to appreciate the plasticity of these cells and to intervene in the phenotypic changes they undergo in various pathological disorders. Many factors have been reported to regulate phenotypic modulation or some part of SMC differentiation by activating transcription of SMCspecific genes. These can be biochemical factors, including different hormones and growth factors, or physical factors, including mechanical stimuli and cell-cell interactions ${ }^{169-171}$ (figure 1.7).

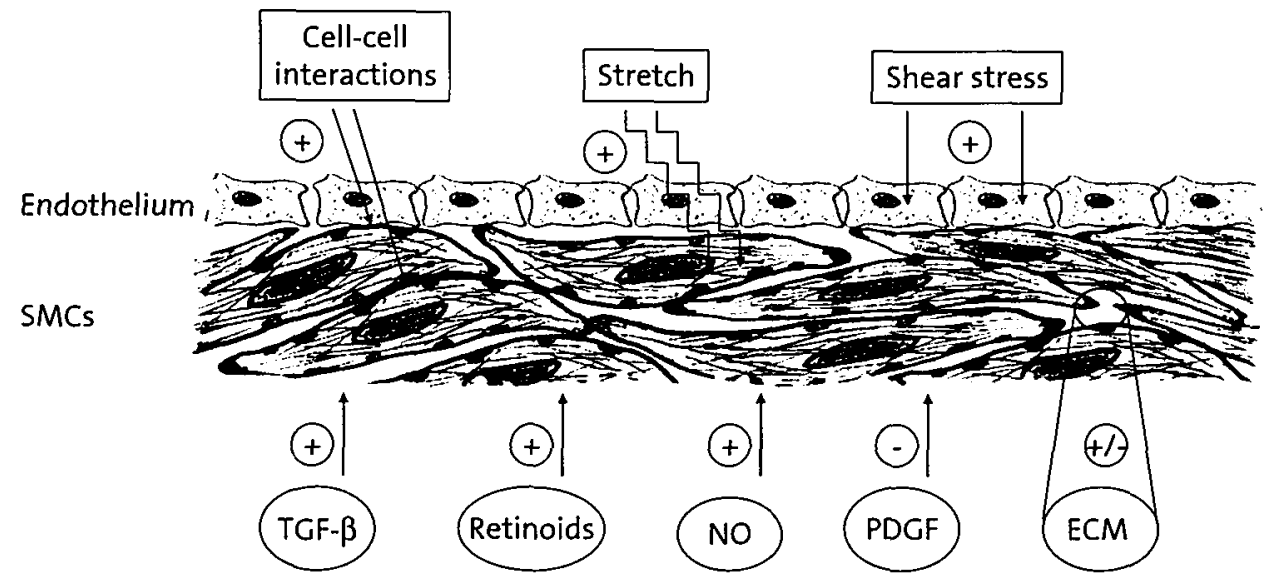

Figure 1.7 The effects of various factors that are involved in SMC development, differentiation, and phenotypic modulation on the contractile SMC phenotype.

\section{Biochemical factors}

The list of biochemical factors that influence SMC phenotype or SMC differentiation is long. Among these factors are ECM proteins, cytokines, and growth factors. In this review, the focus will be on an arbitrary selection of factors that are of generally accepted importance for SMC differentiation and are backed by a substantial amount of experimental data. These factors include PDGF, TGF- $\beta$, retinoids, nitric oxide (NO), and heparin and other ECM compounds. Besides the factors discussed here, compounds like $\mathrm{FGF}^{172}$, insulin-growth factor-1 and $-11^{173}$, endothelin- $1^{174}$, and angiotensin $11^{175}$ have been shown to affect SMC phenotype. 


\section{PDGF}

Two different PDGF molecules have been identified that are of significance for SMC differentiation, designated PDGF-A and PDCF-B ${ }^{176}$. PDGF molecules form homoand hetero-dimers, which bind to PDGF receptors (PDGFR). In the early phases of SMC development, PDGF signaling is critical for endothelial cells to recruit SMC precursors ${ }^{177}$. Mesenchymal cells surrounding endothelial cells during embryogenesis express PDGFR- $\beta$ and respond to endothelium-derived PDGF-B by proliferation. Consequently, PDGF-B-, PDGFR- $\alpha$-, and PDGFR- $\beta$ knockout mice have reduced numbers of $S M C s^{178,279}$. PDGF isoforms are also known to regulate SMC proliferation and migration after arterial injury in adults ${ }^{180,181}$. In view of the link between SMC proliferation/migration and the synthetic phenotype, it would be expected that PDGF affects phenotypic modulation of SMCs. Indeed, PDGF-B has been shown to induce phenotypic modulation of contractile pig coronary artery SMC clones towards the synthetic phenotype ${ }^{3}$. Interestingly, there are marked differences in the response of different SMC subsets in vitro to PDGF-B treatment. For example, SMCs with rhomboid or epithelioid morphology show a higher increase of DNA synthesis compared to spindle-shaped SMCs ${ }^{155}$. Thus, PDGF-B not only promotes phenotypic modulation from the contractile to the synthetic end of the spectrum, but also activates SMCs that already have a synthetic phenotype.

\section{TGF- $\beta$ and TGF- $\beta$-related molecules}

TGF- $\beta$ isoforms are essential for induction of SMC differentiation in vivo. They appear to play a role in the migration of SMC precursors to endothelial or epithelial cells and the subsequent interactions between them, as revealed by different TGF- $\beta$ knockout mice ${ }^{182-184}$. In vitro, the importance of TGF- $\beta$ for the early phases of SMC development is also evident. Cultured neural crest cells and other multipotent cells like embryonic 10T1/2 cells differentiate towards spindle-shaped SMCs when treated with TGF- $\beta^{170,185}$. In adult tissues, TGF- $\beta$ isoforms also promote contractile SMC differentiation ${ }^{182}$. For example, TGF- $\beta_{1}$ increases $\alpha-S M A, S M-M H C$, and SMcalponin levels in cultured SMCs ${ }^{186}$. TGF- $\beta_{2}$ stimulation of cultured porcine SMCs increases $\alpha$-SMA and desmin levels, but does not affect expression of more advanced contractile phenotype markers like $S M-M H C^{3}$. In addition, it suppresses the degradation of matrix proteins through inhibition of ECM-degrading proteases and increased synthesis of their inhibitors ${ }^{187}$. On the other hand, TGF- $\beta_{1}$ has also been reported to stimulate synthesis of proteins like collagen, elastin, and fibronectin, which is associated with the synthetic SMC phenotype ${ }^{188,289}$. The net effect of TGF- $\beta$ isoforms however is promotion of the contractile phenotype. In line with this, TCF- $\beta$ also induces cell cycle proteins like p21 and p15, which suppress cell division, and increases levels of transcriptional regulators like cardiac ankyrin repeat protein (CARP), which inhibits DNA synthesis ${ }^{190}$.

Downstream signaling of TGF- $\beta$ is mediated by different TGF $-\beta$ receptors which phosphorylate SMAD signal transduction proteins. Phosphorylated SMAD complexes translocate to the nucleus and interact with transcription factors or 
transcriptional cofactors, thereby regulating gene expression ${ }^{191}$.

A TGF- $\beta$-like factor which has an impact on SMC phenotype is activin A. It binds to different activin receptors, thereby regulating differentiation, also via SMADs ${ }^{192}$. Activin A stimulates expression of contractile marker proteins like $\alpha$-SMA and $S M 22 \alpha$, but does not affect migration. The effect of activin A on SMC proliferation is unclear, since both an induction of DNA synthesis and the lack thereof have been reported ${ }^{167,193}$. Like TGF- $\beta$, activin $A$ also induces CARP expression ${ }^{194}$. Of note, transdifferentiation of endothelial cells to SMCs in culture has been shown to be mediated by activin $\mathrm{A}^{140}$.

In conclusion, the different isoforms of TGF- $\beta$ stimulate SMC differentiation towards a contractile phenotype by increasing contractile protein levels, inhibiting proliferation, and stabilizing the ECM.

\section{Retinoids}

Several in vitro models of early SMC differentiation have shown that differentiation towards SMCs is retinoic acid (RA)-dependent ${ }^{169,195}$. RA is particularly implicated in the induction of neural crest-derived SMC differentiation, but cells of embryoid bodies cultured in RA-containing medium are much more likely to differentiate into SMCs as well ${ }^{113,196}$.

Together with all-trans retinol and related molecules, RA belongs to the class of retinoids, which are regulatory signaling molecules. Transport, metabolism, and levels of RA and all-trans retinol are regulated by cellular RA-binding proteins (CRABP) and CRBPs respectively. Free RA can bind to RA receptors (RAR) or retinoid $X$ receptors (RXR). Subsequently, RA/RAR or RA/RXR complexes interact with other transcriptional regulators and bind to RA response elements in promoters of RA responsive genes ${ }^{197,198}$.

As a result, both the synthetic and the contractile SMC phenotype can be promoted, depending on the systems that are activated. This becomes apparent in changes of proliferative potential| ${ }^{199}$ and migration properties ${ }^{24}$. In addition, expression of contractile SMC markers like $\alpha-S M A$ and $S M-M H C$ is increased upon RA treatment, but CRBP-1, a marker of synthetic SMCs, is also transcriptionally induced by $R A^{18,24,200}$. RA lowers $S M C$ migration potential through induction of specific ECM proteins and inhibition of matrix degrading enzymes like collagenase $e^{200,201}$. Although it has been reported that retinoids stimulate SMC proliferation, the majority of studies indicates that retinoids decrease SMC proliferation by either targeting phosphorylation of $R b$ or by increasing p21 levels ${ }^{199,202}$. Interestingly, RA is also a regulator of PDGF-B and TGF- $\beta_{1}$ expression, exemplifying the crosstalk between the different factors that shape the SMC phenotype ${ }^{197}$.

While the effects of RA on SMC phenotype in vitro are variable, the response of SMCs to RA treatment in vivo usually consists of modulation towards the contractile phenotype ${ }^{203}$. 


\section{Nitric oxide}

There is increasing evidence that nitric oxide (NO) is an important regulator of vascular SMC phenotype ${ }^{204-206}$. NO is generated by three distinct NO synthases: neuronal, inducible, and endothelial NO synthase $(e N O S)^{207}$. eNOS is the predominant isoform in the vasculature. Physiological levels of NO, generated by eNOS, promote the contractile SMC phenotype. in particular, NO appears to target SMC proliferation. Increased NO production by eNOS gene transfer has been shown to inhibit neointimal SMC growth ${ }^{208}$. Moreover, injury of the arterial wall of eNOS mutant mice leads to a higher degree of SMC hyperplasia compared with wild type mice ${ }^{209}$. NO has also been found to suppress synthesis of ECM proteins ${ }^{210}$. How NO regulates SMC phenotype mechanistically is presently unclear. It has been suggested that it may act via modulation of the mitogen activated protein kinase pathway, which promotes the synthetic SMC phenotype, or the phosphatidylinositol 3-kinase pathway, which promotes the contractile SMC phenotype ${ }^{211}$. In addition, there is evidence for inhibitory effects of NO on nuclear factor kappa $B(\mathrm{NFKB})^{212}$ and $\mathrm{AP}-1^{213}$, which are growth-related transcription factors. Finally, NO may directly target cell cycle proteins, thereby preventing cell cycle progression ${ }^{214}$.

\section{Extracellular matrix components}

As stated before, the local environment is one of the key determinants of the SMC phenotype. As such, the ECM in which SMCs are embedded has important effects on SMC phenotype. For example, heparin, an ECM component belonging to the group of the proteoglycans, has been shown to promote the maintenance of a contractile phenotype and to slow down SMC proliferation. Its mechanism of action is unclear, but it may act at the posttranscriptional level since $\alpha$-SMA protein levels increase upon heparin stimulation whereas mRNA levels are stable ${ }^{38}$. In rat SMCs, desmin and SM-MHC levels are not affected by heparin stimulation. In contrast, porcine SMCs with a spindle-shaped morphology have increased desmin levels after heparin stimulation ${ }^{3}$. Some characteristics of SMCs such as age and morphology appear to influence the effects of heparin. For example, bovine spindle-shaped SMC proliferation was not inhibited by heparin treatment, in contrast to rhomboid SMC proliferation ${ }^{155}$. However, porcine SMC proliferation was inhibited by heparin, irrespective of morphology ${ }^{3}$.

Other ECM components also provide good examples of the complex relation between ECM composition and SMC phenotype. For instance, fibrillar collagen type I has been shown to promote the contractile phenotype of SMCs whereas monomeric collagen type I activated SMC proliferation ${ }^{215}$. When comparing SMCs cultured on either monomeric or polymerized collagen, great differences in gene expression profile were observed ${ }^{216}$. Many differentially expressed genes were ECM or cytoskeletal proteins. Several genes that were downregulated in SMCs grown on fibrillar collagen were upregulated after balloon injury of the rat carotid artery, indicating that SMC phenotypic responses to ECM composition can be adequately 
studied in vitro ${ }^{216}$. The different forms of collagen type I modulate integrin signaling and SMC-responsiveness to PDGF- $\mathrm{B}^{217}$. The composition of the collagen fibrils also influences the migration properties of the SMC. This is associated with a different focal adhesion composition and integrin function ${ }^{215,218}$.

Like fibrillar collagen type I, laminin and collagen type IV have been shown to promote the contractile phenotype. In contrast, fibronectin stimulates modulation towards a synthetic phenotype ${ }^{16,219-221}$. Expression of a specific splice-variant of fibronectin, which includes the so-called extra domain $A$ has been proposed to be associated with SMCs of the synthetic phenotype ${ }^{222}$.

Not only the composition, but also the organization of the ECM modulates SMC phenotype. This becomes clear in three dimensional (3D) culture systems, which are likely to be a better representation of the in vivo situation than conventional two dimensional (2D) systems. SMCs in a 3D collagen matrix are less proliferative compared to SMCs cultured on a $2 D$ collagen matrix ${ }^{223}$. On the other hand, $\alpha-S M A$ levels are diminished in the 3D system, indicating a less contractile phenotype. These results have been extended by showing that SMC responses to PDGF-B, heparin, and TGF- $\beta_{1}$ were affected by ECM organization ${ }^{224}$.

Modulation of SMC phenotype by ECM proteins is thought to be mediated by their binding to specific integrin receptors. The integrins are at the junction of the ECM and the $S M C$, and pass on the spatial arrangement of the ECM to the SMC, thereby activating focal adhesion kinases and altering cytoskeletal organization ${ }^{225}$. Moreover, the ECM is a reservoir of cytokines and growth factors that are bound to specific ECM components ${ }^{226}$. Release of these factors also affects SMC phenotype. The in vitro studies indicate that both the composition and the organization of the ECM have major consequences for SMC phenotype. Because of the great number of ECM compounds and the various integrin combinations that mediate the phenotypic changes, much work needs to be done to establish the overall effects of the ECM on SMC phenotype in vivo.

\section{Physical factors}

In addition to the biochemical environment, the physical environment is important for shaping the SMC phenotype. Physical factors like cell-cell interactions, pressure, and flow are known to have profound effects on SMC phenotype and induction of SMC differentiation. The best example of the effects of the physical environment on SMC phenotype is probably provided by SMCs of the aorta, which experience a markedly different physical environment depending on the distance from the heart. The first segment of the aorta has to withstand great pressure caused by the pumping of the heart. Consequently, SMC morphology and ECM composition in this segment differ from the abdominal segment, which has to comply with the high pressure and flow generated by the heart to a much lesser extent. 


\section{Cell-cell interaction}

Interactions between mesenchymal cell precursors and epithelial cells regulate differentiation and spatial organization of visceral SMCs. Using tissue recombinants, epithelial cells from various origins were shown to be able to induce $\alpha$-SMA expression of uterine and bladder mesenchyme ${ }^{171,227}$. In addition, epithelialmesenchymal cell contact has been shown to be pivotal for SMC differentiation in the lung ${ }^{228}$. Vascular SMC precursors also appear to depend on physical interaction with endothelial cells for acquiring a contractile phenotype, as shown by cocultures of 10T1/2 multipotent embryonic cells and endothelial cells ${ }^{170}$. The 10T1/2 cells increased their contractile marker gene expression, and acquired a spindleshaped morphology when they were allowed to contact the endothelial cells. This effect was inhibited by TGF- $\beta$ antibodies. PDGF-B inhibition also prevented migration of the $10 T 1 / 2$ cells to the endothelial cells, indicating that physical and chemical factors converge at some point controlling the SMC differentiation program. Further augmentation of contractile SMC marker expression was obtained when $10 \mathrm{~T} 1 / 2$ cells were implanted in mice, probably because they encountered the appropriate physical and biochemical environment.

Co-culturing of adult SMCs and endothelial cells leads to different results. For example, contractile spindle-shaped porcine SMCs modulated towards less contractile rhomboid SMCs when co-cultured with endothelial cells from the coronary artery ${ }^{3}$. Concurrent with the change in morphology, $\alpha$-SMA and SM-MHC expression was reduced. Thus, mature contractile SMCs respond in a different way to endothelial signals as compared to developing SMCs. It should be emphasized that the adult endothelial cells that are generally used in culture are not quiescent but rather resemble injured endothelium. Consequently, these activated cells may have different effects on SMC differentiation when compared with the normal interactions in vivo and during development.

It is well known that interactions between SMCs stabilize the contractile phenotype. For example, cultured gastro-intestinal SMCs modulate to a lesser extent when plated at high density ${ }^{16}$.

It is clear that interactions between SMCs themselves and between SMCs and the cells that are lining them are necessary for appropriate SMC differentiation. It is also obvious that these interactions are difficult to mimic in culture, hampering the study of molecular interactions that are involved. However, it appears that cell-cell interactions are particularly required for induction and maintenance of the contractile phenotype. Loss of cell-cell contacts may promote modulation towards a synthetic phenotype by allowing migration and proliferation.

\section{Cell shape}

Visceral SMC precursors consistently turn from round to elongated before SMC marker protein expression starts ${ }^{228}$. Epithelial cells may promote this mesenchymal cell elongation by producing a basement membrane on which they can attach, 
spread and elongate. Alternatively, growth of the epithelial cells and the lumen of the tubes they line may cause elongation of surrounding mesenchymal cells. The mechanical tension generated by cell spreading and elongation seems to mediate the induction of differentiation. In a tissue culture system where cell rounding was stimulated, undifferentiated mesenchymal cells from intestine, kidney, or lung remained undifferentiated ${ }^{228}$. In contrast, upon culture system-induced elongation, the cells started to express contractile smooth muscle proteins and developed membrane potentials. Induction of differentiation in this way was independent of cell proliferation. Biochemical factors like TGF- $\beta_{1}$, PDGF-B, and RA were unable to influence SMC marker gene expression of the undifferentiated round cells. However, after elongation and differentiation, TGF- $\beta_{1}$ stimulated and PDGF-B decreased marker gene expression. Interestingly, once the cells were elongated, forced adoption of a round shape did not affect SMC marker expression anymore. Since the shape of the cell is largely determined by the cytoskeleton and changes in cytoskeletal organization are mediated by integrins, it is likely that changes in integrin expression play a role in the early induction of SMC differentiation ${ }^{229}$.

\section{Pressure and flow}

SMCs continuously encounter mechanical stimuli which have dramatic effects on their phenotype. Pressure causes stretch (tensile stress) and flow causes shear stress, both of which induce vessel wall remodeling by changing SMC morphology, proliferation, and gene expression. The effects of shear stress are mediated by the endothelium, for example through NO release, which modulates the response of SMCs to this type of mechanical stress. Stretch, in contrast, acts directly on the SMCs.

Mechanical forces enhance the expression of both ECM and contractile proteins by SMCs in the vessel wall. This is already apparent in the developing embryo, where ECM synthesis and contractile SMC differentiation coincide with the onset of pressure in the embryonic circulation ${ }^{230}$. Application of mechanical strain on adult aortic SMCs leads to increased collagen and fibronectin synthesis and build-up of $E C M$, even though matrix metalloproteinase (MMP) activity is also increased ${ }^{231}$. Likewise, stretch of the adult rat bladder stimulates expression of collagen type I and III and is accompanied by coordinate changes in integrin expression ${ }^{232}$. Integrin- $\beta 1,-\beta 3$, and $-\alpha v$ levels increase, whereas integrin- $\alpha 1$ expression declines. Also, inhibition of integrin function attenuates stretch-induced SMC proliferation. It is clear that through their function in both early SMC differentiation induction as well as the transduction of mechanical stress, integrins are important integrators of the physical factors that affect SMC phenotype. Considering the importance of matrix-integrin interactions for the activation of signal transduction pathways that regulate SMC phenotype, changes in ECM composition are likely to be of major significance for the SMC response to mechanical stimuli. Indeed, cyclic mechanical strain applied on neonatal rat SMCS cultured on different ECM substrates has been shown to have differential effects on for example SM-MHC expression ${ }^{233}$. 
Interestingly, the response of adult SMCs was not dependent on the type of matrix on which they were cultured. In another study, mechanical strain applied to SMCs grown on laminin caused a greater increase in h-caldesmon when compared to SMCs grown on collagen type I or IV ${ }^{234}$. The signal transduction pathways that are activated upon stretch and shear stress ${ }^{235}$ have also been shown to increase expression of $\alpha$-SMA and $h$-caldesmon, whereas SM-calponin levels are not affected $^{234,236-238}$.

Conflicting data exist on the growth response of SMCs to mechanical strain in culture. Both increased and reduced proliferation have been reported ${ }^{238,239}$. This can probably be attributed to differences in the degree and characteristics of stretch that was applied and the initial degree of differentiation of the cells. Thus, these contradictory findings may actually reflect both the intrinsic SMC heterogeneity as well as the heterogeneity caused by a different local environment.

All in all, mechanical stress clearly has a major impact on SMC phenotype in vivo. It appears that physiological stress promotes the contractile SMC phenotype, whereas over- or under-stressing provokes modulation towards the synthetic phenotype. In vitro studies addressing this issue are hampered by the lack of a defined system that mimics the stretch encountered in vivo.

\section{Models for studying transcriptional control of smooth muscle cell differentiation and phenotypic modulation}

Those genes encoding the marker proteins that shape the contractile phenotype of the SMC have also been used in studies aimed at unraveling the transcriptional pathways that are underlying the phenotypical plasticity of the SMC. The best studied genes are $\alpha$-SMA, SM-MHC, SM22 $\alpha$, SM-calponin, telokin, and $\gamma$-SMA. Mostly, classical and straightforward reporter gene assays have been performed, in which the effects of successive deletions and point mutations of putative cis-acting elements on promoter activity were assessed. Identification of proteins that regulate SMC promoter activity has also been approached by established techniques like footprinting and gel-shift assays. Both in vitro cell culture models and in vivo models e.g. transgenic or knockout mice have been employed and have contributed to our understanding of expression regulation in SMCs. Recently, micro array studies have been used for analyzing differential expression of genes between SMCs of different phenotypes or at different stages of differentiation ${ }^{223}$. $V i a$ this approach, new transcription factors or signal transduction molecules that influence SMC phenotype or differentiation may be identified. Below, common cell culture systems and mouse models that have been developed to study the regulation of SMC differentiation and phenotypic modulation will be discussed. 


\section{In vitro models}

The use of primary SMC cultures for studying transcriptional regulation of phenotypic modulation and differentiation is complicated because of SMC heterogeneity. It is for example not known if one particular phenotype of SMCs is more easily transfected than another. Also, there is active modulation towards the synthetic phenotype during the early passages, leading to an unstable experimental situation. A way of circumventing these problems is to establish a homogeneous population of SMCs by cloning primary SMCs. However, the finite lifespan of such cloned SMCs prevents comparison of results between different research groups. Therefore, a big improvement in the study of SMC differentiation regulation in vitro would be provided by the development of a reliable and stable system that is capable of maintaining SMCs in a contractile state, or, even better, to manipulate phenotypic modulation in a reversible way. In the present primary cell systems, the degree of modulation after culturing for longer periods is too variable, depending on the length of time in culture, the way of isolating the SMCs, serum concentration, and cell density.

Several cell lines have proven useful for studying mechanisms of transcriptional regulation of SMC differentiation, in addition to ES cells and embryoid bodies. These include PAC1, A10, A7r5, Monc1, and A404 cells, which have different differentiation characteristics as assessed by the marker proteins they express. Because of these different expression profiles, comparing results obtained by using several of these cell lines may help to delineate factors that are involved in SMC differentiation. However, these cells do not contract and hence cannot be used to investigate factors that induce the full contractile phenotype. Also, variations in terms of morphology and SMC marker expression is encountered between the different cell lines in different labs, complicating comparison of results. Nevertheless, the combined results of studies using these cell lines as a model for SMC differentiation or phenotypic modulation have provided many leads to pursue in in vivo studies.

Monc1 and A404 cells are derived from multipotent cells and have been proposed to serve as a SMC differentiation model. Monc1 cells are cells from neural crest origin which have the potential to differentiate into SMCs in vitro ${ }^{185.240}$. After induction of differentiation, these cells express SM-MHC and thus represent SMCs with a relatively contractile phenotype. A404 cells originate from the embryonic carcinoma cell line P-19, which can be induced to form SMC-like cells with RA ${ }^{169}$. Originally, only a small proportion of the cells differentiated towards SMCs ${ }^{241}$. Later, this system was optimized to enrich differentiation towards SMCs by using a selection marker under the $\alpha$-SMA or SM-MHC promoter ${ }^{195}$. The resulting A404 cells were spindle-shaped before and after differentiation, and had a contractile mRNA expression profile. Also, expression of transcription factors that have been 
shown to regulate SMC differentiation, like myocardin, was induced by RA treatment of these cells $\mathrm{s}^{242}$.

A10 as well as A7r5 cells have been derived from the thoracic aorta of embryonic rats $^{243}$. Although they have a common origin, they exhibit completely different characteristics, underlining the heterogeneity of SMCs in the vessel wall. A10 cells are epithelioid SMCs that express many markers of SMC differentiation but nonmuscle myosin instead of SM-MHC ${ }^{244}$. A7r5 cells are widely used spindle-shaped SMCs with a more contractile phenotype. They express SM-MHC to a variable extent. PAC 1 cells originate from a culture of adult rat pulmonary artery $S M C \mathrm{~s}^{245}$. They express high levels of SM-calponin, $\alpha-S M A$, and SM22 $\alpha$, but little SM-MHC, and proliferate more rapidly than $A 7 r 5$ cells $^{246}$.

An in vitro model of SMC phenotypic modulation which has not been used so far to investigate transcriptional pathways makes use of transgenic mice harboring a temperature-sensitive SV4O large T-antigen gene ${ }^{247}$. Aortic SMC lines from these mice can be induced to express SM-MHC and SM-calponin and to slow down proliferation.

\section{In vivo models}

Although they are useful, it is unlikely that the rather homogeneous SMC lines described above are representative of the diverse SMC populations that are found in vivo. Also, factors like flow and pressure, cell-cell interactions, and ECM composition cannot be accurately imitated in vitro. Since these factors affect SMC differentiation in many ways, complementation of in vitro results with data obtained from in vivo models is required to identify the pathways involved in SMC differentiation.

There is a large number of reports on the regulation of SMC marker genes in vivo, summarized in table 1.2. These studies have revealed that the so-called CArG box (see page 44 ) is one of the critical elements for promoter activity in SMCs. In addition, expression of the tested reporter genes was frequently detected in specific subsets of SMCs, i.e. only in arterial or venous SMCs, or only in visceral SMCs. This indicates that there are distinct transcriptional programs that direct expression in different SMC types. It appears that expression of SMC marker genes in visceral and venous SMCs requires additional cis-elements as compared to arterial $S M C s^{94,248}$. The differences between expression patterns in venous and arterial SMCs show that this is not related to the mere embryological origin of the SMCs. In numerous studies, heterogeneous expression of reporter genes within tissues was observed, in line with the heterogeneity of endogenous gene expression patterns in SMCs. Remarkably, many of the tested promoter fragments that had been shown to be sufficient for driving expression of a gene in vitro were not active at all in vivo. Besides the discrepancy between in vitro and in vivo conditions, many of these problems may be explained by features inherent to the 
small size of the used promoter fragments, which makes them more prone to influences from cis-elements around the site of integration and to silencing by methylation ${ }^{249}$. In addition, cis-elements with cell type-specific enhancing activities can be far apart from the basal promoter. Hence, in transgenic mice the use of large bacterial artificial chromosomes (BAC)s, P1 artificial chromosomes (PACs), or yeast artificial chromosomes (YACs) is preferred over the use of small promoter reporter constructs for studying transcriptional regulatory elements. These large sequences are much more likely to contain not only all the regulatory elements but also the information for the correct higher order chromatin structure which is known to influence gene transcription profoundly. The feasibility of this approach has been shown by studies using BACs containing the SM22 $\alpha$ and SM-calponin loci $^{250,251}$. Transgenic mice containing these BACs showed an expression pattern that exactly mimicked the pattern of the endogenous gene. Another way of overcoming the limitations of the use of small promoter reporter constructs is by generating knockin mice with a reporter gene inserted in the endogenous gene. In this way, all the regulatory DNA sequences on the chromosome are present. This approach has been successfully applied with the $S M 22 \alpha$ gene $^{68}$. The contribution of a specific cis-element to gene expression can be demonstrated by making knockin mice that harbor a mutated cis-element in the context of an otherwise intact gene. To our knowledge, this type of experiment has not been performed with SMCspecific genes so far. Integration of promoter activity over time by using a creactivated LacZ transgene and a cre recombinase driven by the SM-MHC promoter has solved problems encountered with the possible episodic expression of this gene, and may be useful for other genes as well ${ }^{259}$.

The contribution of some transcription factors to regulation of SMC differentiation has been evaluated by generating mice that are deficient for these factors. Most of these knockouts were embryonically lethal, presumably because the factors that were targeted are widely expressed and have many additional functions in other cells. Examples include SRF knockout mice, which exhibit a severe gastrulation defect and complete absence of mesodermal cells ${ }^{263}$, myocardin knockout mice, which die at E10.5 ${ }^{115}$, and GATA-6 null mice which die at E6.5-E7.5 because of defective visceral endoderm formation leading to extra-embryonic development ${ }^{264}$. MEF2C knockout mice develop complex vascular malformations, partly because of a loss of mesenchymal cells ${ }^{265}$. Conditional SRF knockout mice have been developed but thus far not used to investigate SMC-specific effects of SRF deficiency ${ }^{266}$. These mice will also allow analysis of the effects of SRF deficiency later in development. In summary, the results from transgenic mice indicate that the promoters of SMCspecific genes can be used to direct expression specifically in SMCs, provided that a large promoter segment is used. It appears that many SMC-specific promoters contain modules that direct expression of the gene in specific SMC subsets. Apart from the CArG box, the exact nature and location of cis-elements that contribute to or are critical for SMC-specific expression of these genes however remains largely unknown. 
Table 1.2 Expression pattern of reporter genes driven by indicated SMC gene promoter fragments in transgenic mice. If the effect of the mutation of specific elements on the expression pattern was studied, this is indicated in the column 'mutation element'. \# of lines indicates the number of founder

\begin{tabular}{|c|c|c|c|c|c|c|}
\hline Gene & Promoter fragment & $\begin{array}{l}\text { Mutation } \\
\text { element }\end{array}$ & $\begin{array}{l}\text { Stage } \\
\text { tested }\end{array}$ & Reporter & $\begin{array}{l}\text { \# of } \\
\text { lines }\end{array}$ & Ref. \\
\hline \multirow[t]{3}{*}{ Mouse ACLP } & -2502 to +176 & $\cdot$ & Adult & $\operatorname{lac} Z$ & 3 & 96 \\
\hline & & $\therefore$ & E11.5 & $\operatorname{lac} Z$ & $?$ & 120,252 \\
\hline & -1343 to 0 & CArGfar & E11.5 & $\operatorname{lac} Z$ & 4 & 252 \\
\hline \multirow{11}{*}{$\begin{array}{l}\text { Human } \\
S M 22 \alpha\end{array}$} & & CArGnear & E11.5 & $\operatorname{lac} Z$ & 16 & 252 \\
\hline & -445 to +1 & - & E10.5 & $\operatorname{lac} Z$ & 3 & 250 \\
\hline & -445 to + intron 1 & - & $\mathrm{Eg} / 10.5$ & lacz & 5 & 250 \\
\hline & $150 \mathrm{~kb} B A C$ & - & E13.5 & NA & $?$ & 250 \\
\hline & DJUKU DAC & - & Adult & NA & $?$ & 250 \\
\hline & -441 to +41 & - & E11.5 & $\operatorname{lac} Z$ & 6 & 259,254 \\
\hline & -280 to +41 & - & E11.5 & $\operatorname{lac} Z$ & 7 & 259 \\
\hline & -5000 to +41 & - & E11.5 & $\operatorname{lac} Z$ & 2 & 259 \\
\hline & & both CArGs & E11.5 & $\operatorname{lac} Z$ & 12 & 259,254 \\
\hline & & CArGfar & E11.5 & $\operatorname{lacz}$ & 4 & 254 \\
\hline & -441 lo +41 & CArGnear & E11.5 & lacz & & 254 \\
\hline \multirow{8}{*}{$\begin{array}{l}\text { Mouse } \\
S M 22 \alpha\end{array}$} & & $Y Y_{1}$ & E11.5 & $\operatorname{lac} Z$ & 2 & 259 \\
\hline & -445 to +65 & - & E9.5-15.5 & lacz & 2 & 248 \\
\hline & -2126 to +65 & - & E9.5-15.5 & lacz & 3 & 248 \\
\hline & $-212000+03$ & - & Adult & lacz & $?$ & 248 \\
\hline & -2126 to -4140 & - & Adult & lacZ & $?$ & 248 \\
\hline & -475 to +61 & - & Adult & $\operatorname{lac} Z$ & 9 & 255 \\
\hline & & - & E11.5/Adult & $\operatorname{lacz}$ & $? \geq 2$ & 59 \\
\hline & -445 to +88 & $G / C$-rich element & E11.5/Adult & $\operatorname{lac} Z$ & $? 22$ & 59 \\
\hline \multirow[t]{2}{*}{ Rabbit telokin } & -163 to +147 & - & Adult & SV4O ITa & 3 & 256 \\
\hline & -2250 to +147 & - & Adult & SV40 ITa & 2 & 86,256 \\
\hline \multirow[t]{3}{*}{$\begin{array}{l}\text { Human SM- } \\
\text { calponin }\end{array}$} & $\begin{array}{c}103 \mathrm{~kb} \mathrm{BAC} \\
50 \mathrm{~kb} \text { BAC }\end{array}$ & $\cdot$ & E9.5-adult & NA & 2 & 251 \\
\hline & $13.7 \mathrm{~kb}(p+i 1)$ & - & E15.5-adult & CAT & 5 & 64 \\
\hline & $11.6 \mathrm{~kb}(p+i 1)$ & - & adult & CAT & 6 & 64 \\
\hline \multirow[t]{4}{*}{ Mouse $\gamma$-SMA } & $9.3 \mathrm{~kb}(p+i 1)$ & - & adult & CAT & 3 & 64 \\
\hline & $6 \mathrm{~kb}$ & - & adult & CAT & 2 & 64 \\
\hline & $2 \mathrm{~kb}$ & - & adult & CAT & 3 & 64 \\
\hline & $0.6 \mathrm{~kb}$ & - & adult & CAT & & 64 \\
\hline \multirow[t]{4}{*}{ Mouse Crp2 } & $5 \mathrm{~kb}$ & - & E11.5 & $\operatorname{lac} Z$ & 2 & 92 \\
\hline & $5.5 \mathrm{~kb} 1 \mathrm{~A}$ & - & E16.5 & $\operatorname{lacZ}$ & 2 & 94 \\
\hline & $7 \mathrm{~kb} 1 \mathrm{~B}$ & - & E16.5 & lacz & 2 & 94 \\
\hline & $19 k b(p+i 1)$ & - & E16.5 & lacz & 3 & 94 \\
\hline \multirow[t]{6}{*}{ Mouse Crp1 } & $12 \mathrm{~kb}(\mathrm{p}+\mathrm{i} 1)$ & - & E16.5 & lacz & 9 & 94 \\
\hline & & - & E8.5-E14.5 & $\operatorname{lac} z$ & 6 & 94 \\
\hline & $5 \mathrm{~kb}(\mathrm{i} 1)$ & - & Adult & $\operatorname{lac} Z$ & $?$ & 94 \\
\hline & & CArG box & E12 & lacz & 4 & 94 \\
\hline & -4220 to +88 & - & E13.5-E19.5 & lacz & $?$ & 257 \\
\hline & & - & E13.5-adult & lacZ & 3 & 257,258 \\
\hline \multirow[t]{3}{*}{ Rat SM-MHC } & -4220 to +11600 & & E10.5-adult & Cre-lacz & 3 & 259 \\
\hline & & CArG 1 & E19.5-adult & lacz & 3 & 258 \\
\hline & & CArG 2 & E19.5-adult & lacz & 5 & 258 \\
\hline
\end{tabular}


lines tested. Ref.-literature reference; WT-wild type; CAT-chloramphenicol transferase; SV40 ITa- SV40 large T antigen; Cre-lacZ-Cre recombinase-activated lacZ; p-promoter; i1-intron 1; BAC-Bacterial artificial chromosome; bp-basepairs; kb-kilo basepairs; E11.5- embryonic day 11.5; NA-not appropriate

\section{Expression}

Venous and arterial SMCs; arterioles; skeleton; dermal skin layer

Arterial vasculature; heart tube; somites

See WT; less in somites/trabeculae heart

No expression

Arterial SMCs; somites; cardiac outflow tract

Somites; cardiac outflow tract/ Arterial SMCs; somites; cardiac outflow tract

All SMCs (arterial, venous, visceral organs)

All SMCs (arterial, venous, visceral organs)

Dorsal aorta and branch arteries; myotome of somites; cardiac outflow tract; future right ventricle

See -441 to +41 bp; patchy in dorsal aorta and branch arteries

See -441 to +41 bp

No expression

See -441 to +41 bp

See -441 to +41 bp; no staining somites, less in aorta

No expression

Arterial SMCs; developing heart; myotome of somites

See -2126 to +65 bp

Arterial SMCs

Arterial SMCs

Arterial SMCs; venous SMCs; gut, bladder, bronchi; right atria, part of right ventricle Arterial SMCs; myotome of somites; cardiac outflow tract; future right ventricle

See -445 to +88 ; decrease of expression after injury is attenuated

Bladder; digestive tract; lung; vascular SMCs

Bladder; digestive tract; lung; vascular SMCs; uterus

Adults: bladder; intestine; stomach; uterus; lung; vascular SMCs.

E9.5: heart and dorsal aorta. E14.5: visceral SMCs

All SMCs

All SMCs

Gastrointestinal and urogenital SMCs (low expression)

Gastrointestinal and urogenital SMCs (low expression)

No expression

No expression

SMCs of developing vasculature

No expression

No expression

Vascular SMCs

Vascular SMCs

Arterial SMCs; cardiac outflow tract; myotome of somites; skeletal fibers of body wall; kidney

Arterial SMCs (not in coronary SMCs); cardiac outflow tract; skeletal fibers of body wall (low)

Cardiac outflow tract (low); somites (low)

No expression

Vascular SMCs (not in lungs/cardiac outflow tract; some heterogeneity; embryos: not in large vessels of head \& neck); visceral SMCs (some heterogeneity); esophagus (embryos)

See above; additional expression in bronchi, pulmonary vessels, aorta and umbilical artery; heart, esophagus; heterogeneity absent

No expression

Visceral SMCs and small arteries (decreased expression) 
Table 2.1 (continued)

\begin{tabular}{|c|c|c|c|c|c|c|}
\hline Gene & Promoter fragment & $\begin{array}{l}\text { Mutation } \\
\text { element }\end{array}$ & $\begin{array}{l}\text { Stage } \\
\text { tested }\end{array}$ & Reporter & $\begin{array}{l}\text { \#of } \\
\text { liños }\end{array}$ & meft \\
\hline \multirow{7}{*}{$\begin{array}{c}\text { Rat SM-MHC } \\
\text { Rabbit } \\
\text { SM-MHC }\end{array}$} & -4220 to +11600 & Intronic CArG & E19.5-adult & $\operatorname{lac} Z$ & 4 & 258 \\
\hline & -2305 to -4 & - & Adult & luciferase & 3 & 260 \\
\hline & -547 to +47 & - & E13.5 & lacz & & 261 \\
\hline & -547 to +2784 & - & E13.5 & $\operatorname{lacZ}$ & 8. & 261 \\
\hline & & - & E10.5-E16.5 & $\operatorname{lac} Z$ & $?$ & 261 \\
\hline & & - & $\therefore$ Adult & lacz & 6 & 261 \\
\hline & -2600 to +2784 & CARGA & E13.5/adult & $\operatorname{lac} Z$ & $\geq 5$ & 261 \\
\hline \multirow[t]{6}{*}{ Rat $\alpha$-SMA } & & CArG B & E13.5-adult & lacz & $\geq 5$ & 261 \\
\hline & & Intronic CArG & E13.5 & lacz & $\geq 5$ & 261 \\
\hline & & E-box 1 & E13.5/adult & lacz & 6 & 262 \\
\hline & -2560 to +2784 & $E-b o \times 2$ & E13.5/adult & lacz & 8 & 262 \\
\hline & & E-box 1; E-box 2 & E13.5 & lacz & 3 & 262 \\
\hline & & E-box 1; E-box 2 & Adult & lacz & 7 & 262 \\
\hline
\end{tabular}

\section{The SRF-CArG box axis}

The general picture that emerges from studies employing the promoters of SMC marker genes reveals the central role of the so-called CARC box in the regulation of (a part of) the SMC differentiation program. The CArG box consists of a $C C\left(A / T_{6}\right) G G$ motif. This sequence, or a similar sequence with $C / G$ substitutions in the core, can be found in the promoter of virtually all genes which expression is restricted to SMCs (hereafter referred to as 'SMC-restricted' genes). It may occur in the region upstream of the first exon, but also in introns within the transcriptional unit. Many SMC-specific promoters contain two or more CArG boxes that act in concert. Both the positioning of the CArG box(es) relative to the transcription start site and the sequence of the CArG box have been shown to be important for promoter activity $^{267}$.

The transcription factor that binds to the CARG box is SRF. The major functional domain of SRF is the MADS (MCM1-Agamous-Deficiens-Serum response factor)box, which mediates homodimerization and DNA-binding, and recruits cofactors affecting transcription ${ }^{268}$. Using a dominant negative variant of SRF, it has been shown that differentiation of SMCs is SRF-dependent, although overexpression of SRF in non-SMCs is not sufficient to activate the promoters of SMC-restricted genes $^{51,252}$. SRF levels do not change during phenotypic modulation in vivo, as shown by two models of arterial injury ${ }^{39}$. Thus, although SRF regulates SMC differentiation, it does not regulate phenotypic modulation by itself.

Exactly how a relatively widely expressed transcription factor like SRF controls specific expression of genes in SMCs is still unclear, despite many studies. Recently, an excellent review on this topic has been published ${ }^{268}$, so we will discuss the suggested mechanisms only briefly. First, it has been shown that even though SRF is expressed in many cell types, it is more abundant in the muscle lineages ${ }^{269,270}$. This 


\section{Expression:}

See WT; no expression in large elastic arteries

Vascular SMCs; lung/trachea; intestines; kidney; uterus; bladder (heart in embryos),, $\mathrm{A}^{*}$

Skeletal and cardiac muscle

Skeletal and cardiac muscle; umbilical arteries and abdominal aortä

Smooth, skeletal, and cardiac muscle

All SMCs except uterine SMCs

Smooth (reduced expression), skeletal and cardiac muscle/reduction expression in smaller vessels

No expression

Cardiac and skeletal muscle

Reduced expression in SMCs

Reduced expression in SMCs

No expression

Reduced expression in SMCs

may be relevant because CArG boxes of SMC-restricted genes generally have mutations which lower SRF binding affinity. As a result, these CArG boxes may only be functional when SRF levels are higher. Second, the flanking sequences of the CArG box have been shown to partly determine cell type specificity of reporter gene expression in transgenic mice ${ }^{120}$. Third, cell-type-specific SRF splicing has been observed. This mechanism has been shown to alter the effects of SRF on SMCrestricted genes ${ }^{271}$. For instance, the SRF $\triangle 5 /$ SRF-M isoform, which lacks exon 5, has a lower potential to activate the SM22 $\alpha$ promoter compared to SRF and even suppresses the $\alpha$-SMA promoter in a dose dependent manner ${ }^{166,271}$. Another isoform lacking exons 4 and 5 (SRF-S) is restricted to fully differentiated vascular SMCs, whereas SRF-I lacking exons 3 to 5 is only expressed during embryogenesis and acts as a dominant negative isoform ${ }^{271}$. A fourth possible mechanism leading to SMC-specific transcription by SRF is SMC-specific posttranslational modification ${ }^{272,273}$. SRF can be phosphorylated at its $\mathrm{N}$-terminus, which facilitates DNA-binding of the protein, or at the C-terminus, which attenuates activity of the protein ${ }^{274-276}$. Fifth, cell-type specific competitive inhibition of SRF-binding to the CArG box by another factor recognizing the CArG box or its flanking sequences is yet another mechanism by which SRF activity can be controlled selectively in SMCs. For example, binding of the Yin Yang 1 (YY1) transcription factor to the CArG box has been shown to affect SM22 $\alpha$ promoter activity in synthetic SMCs in culture ${ }^{254}$. Sixth, the subcellular distribution of SRF is tightly regulated. Upon serum deprivation of cultured myocytes, SRF undergoes extranuclear relocation ${ }^{277,278}$. Consequently, SRF transcription activation potential is decreased, leading to diminished SMC promoter activity. However, since serum deprivation of SMCS generally leads to a more contractile phenotype, it is unclear how important this type of regulation is. 
In view of the large body of evidence supporting each of the discussed mechanisms, it is likely that no single mechanism dominates, but rather that the different levels of regulation complement each other.

\section{SRF cofactors}

Recently, another possible mechanism of SRF-mediated SMC-specific gene expression, namely association with smooth muscle selective cofactors, has gained much attention. It has been shown that SRF cofactors determine the effects of SRF on contractile SMC gene expression to a great extent. Several cofactors which contribute to the transcriptional activity of SMC marker genes have now been identified (table 1.3).

Because of its proven contribution to the transcriptional regulation of several genes associated with the contractile SMC phenotype and its critical importance for the development of vascular SMCs ${ }^{115}$, the SRF accessory factor myocardin is of special interest. Myocardin was originally reported to be expressed in cardiac and smooth muscle during development before becoming restricted to the heart in adults. Recently, expression of myocardin was also detected in the adult rat aorta and in visceral SMCs ${ }^{39,242}$. Myocardin stands apart from the other SRF cofactors because it cannot bind DNA by itself ${ }^{168}$. In addition, it contains a SAP (SAF-A/B, Acinus, PIAS) domain, which is involved in chromatin remodeling by recognizing scaffold or matrix attachment regions, thereby regulating transcriptional accessibility. Dimerization of myocardin has been suggested to be required for full transactivation of SMC promoters ${ }^{279}$. Myocardin is a potent activator of transcription, with reported induction of SRF-dependent transcription of several SMC genes by at least nine-fold up to several thousand-fold ${ }^{168,242}$. Knocking down myocardin expression with short interfering RNA or overexpression of dominantnegative myocardin attenuated SM22 $\alpha, \alpha-S M A$, and SM-MHC promoter activity in cultured SMCs ${ }^{242}$. Whereas SRF-levels do not correlate with SMC phenotype, myocardin expression decreased with phenotypic modulation towards a more synthetic state in vitro and in vivo ${ }^{39}$. Recently, the dependence of vascular SMCs on myocardin to initiate an SRF-mediated differentiation program was shown in myocardin null mice ${ }^{115}$. Still, myocardin is not absolutely required for the expression of SMC markers, because SM-calponin expression in cells cloned from rat aorta has been shown to be possible either with or without myocardin expression $^{39}$. Of note, two myocardin related cofactors which also interact with SRF and which are expressed in SMCs have been described, MRTF-A and $-\mathrm{B}^{280}$. These may partially take over the role of myocardin in visceral SMCs.

All in all, the current data indicate that SRF serves as an integrating docking platform, interacting with different co-activators and possibly co-repressors. The specific nature of the SRF-cofactor complex (i.e. the balance between activators and repressors) determines the expression level of those genes dependent on SRF. Cofactors such as myocardin may increase or decrease gene expression, but cannot switch gene expression on or off. Furthermore, since the expression levels of SMC- 


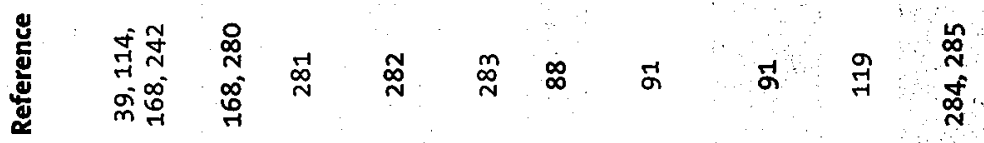

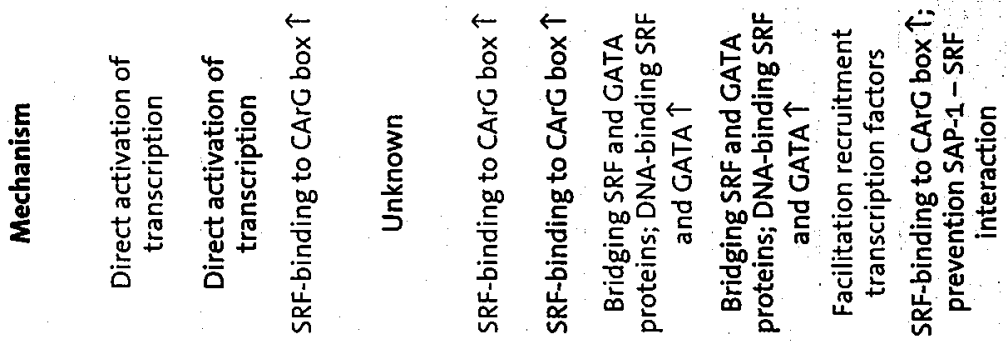

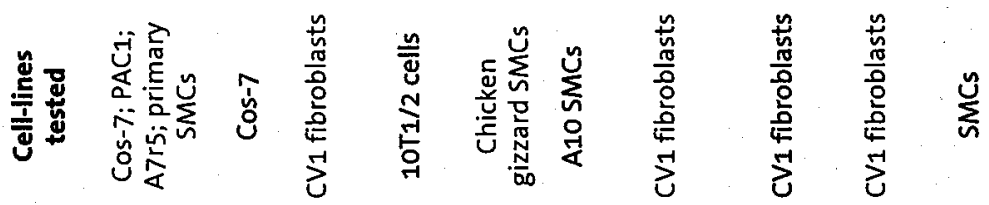

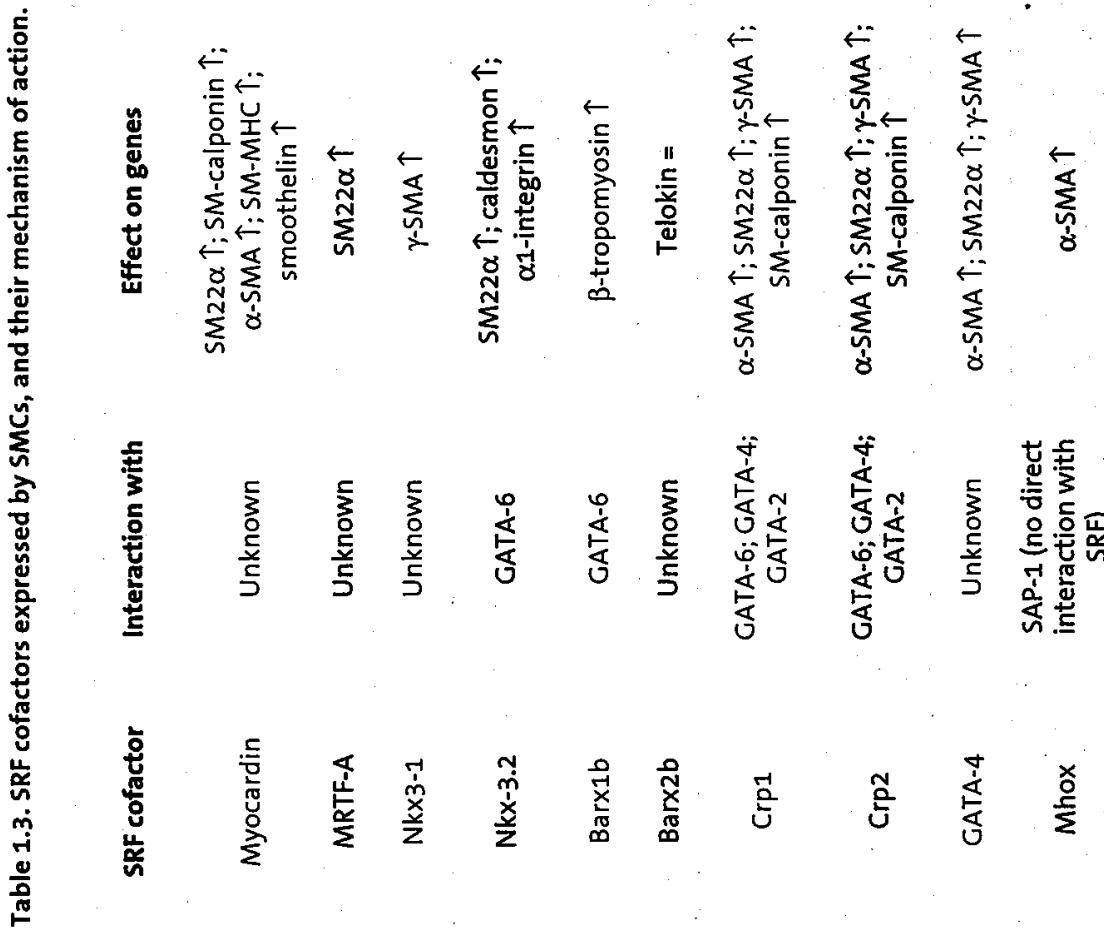

specific genes ultimately define the SMC phenotype, interaction of SRF with different co-activators is likely related to SMC heterogeneity. 


\section{RhoA signaling and SRF}

The best studied upstream factor of SRF is RhoA, a small GTP-binding protein. RhoA has been shown to regulate SRF-dependent promoter activity of SM22 $\alpha, \alpha-S M A$, and SM-MHC via its effector Rho kinase ${ }^{277,286}$. RhoA affects transcriptional activity of these genes in several ways (figure 1.8). It regulates SRF levels and SRF subcellular localization ${ }^{277.278}$. Moreover, RhoA affects actin dynamics which in turn influence SRF-dependent transcription ${ }^{277,287}$. Besides Rho kinase, other RhoA effectors like LIM kinase- 1 are involved in both activation of SRF and stabilization of actin filaments ${ }^{287}$. As such, RhoA-regulated actin filament dynamics appear to play a role in the regulation of the expression of SMC-specific genes. However, it must be kept in mind that RhoA also induces changes in cell morphology, which influence gene transcription as already discussed.

These observations indicate that RhoA is an important regulator of the contractile SMC phenotype. RhoA, which is activated by several factors that affect SMC phenotype like stretch and integrin signaling, may be one of the factors integrating intracellular and extracellular signal $\mathrm{s}^{288}$. In addition, in view of the regulation of SMC contractility by RhoA, it is likely that RhoA signaling plays a central role in relating $S M C$ contractile gene expression to SMC contractile function.

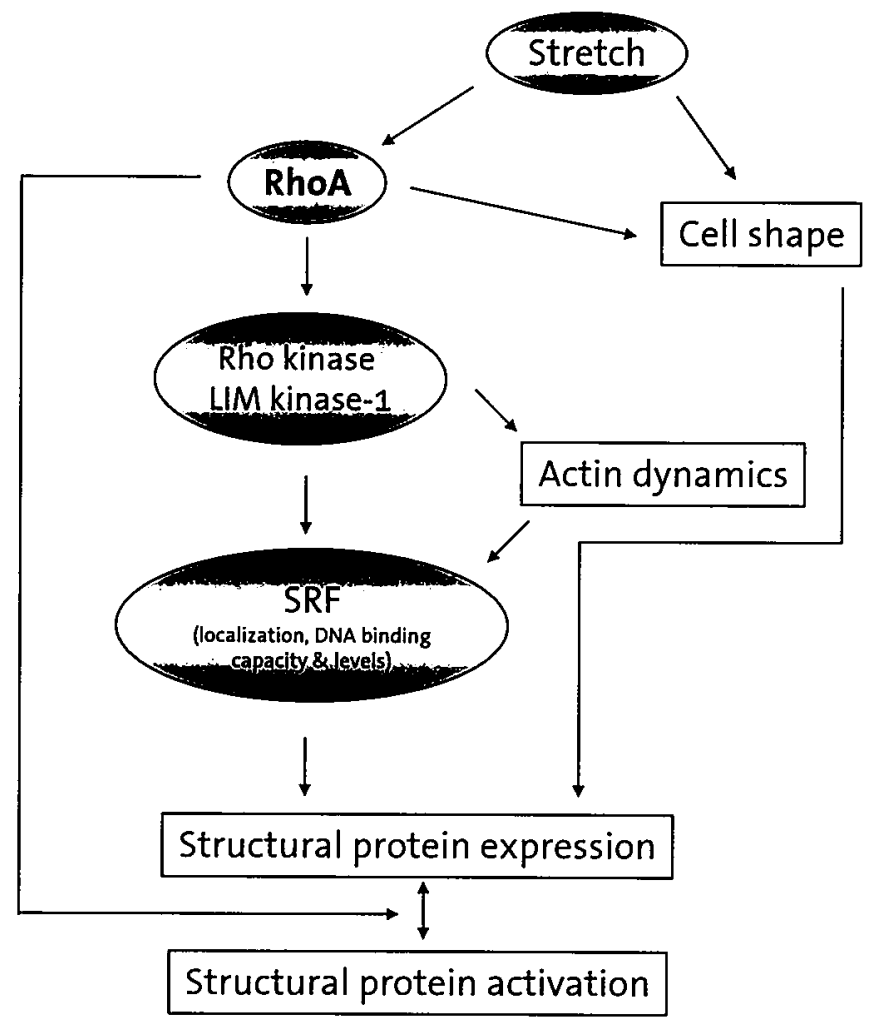

Figure 1.8 Signaling pathways through which RhOA regulates contractile SMC gene expression and contractile SMC function. 


\section{Chromatin remodeling and smooth muscle cell gene expression}

Regulation of gene expression at a higher level, i.e. by chromatin restructuring or DNA modification, is increasingly recognized to be an important regulatory mechanism. This way of regulation, referred to as epigenetics, provides a means for a cell to take its 'history' of gene expression into account ${ }^{289}$. This may be particularly relevant for SMCS, which seem to gradually lose their ability to modulate towards an advanced contractile phenotype later in life. With increasing age, growing numbers of SMCs have encountered stimuli promoting a synthetic phenotype. If this is translated into chromatin modifications as occurs in other cell types, this may change their epigenetic make-up in such a way that a synthetic transcriptional profile persists. These SMCs would then be more specified in their function, i.e. synthetic SMCs. Programmed chromatin remodeling may even be one of the ways in which subsets of SMCs with distinct functions and phenotypes are specified. Via this mechanism, SMCs destined to acquire a synthetic phenotype would get relatively inaccessible 'contractile' genes, whereas these would remain open in contractile SMCs and vice versa. Initially, most SMCs would have open chromatin at both contractile and synthetic loci, retaining their original multifunctional properties which allow them to respond to new stimuli in the appropriate way. In addition, SMCs derived from different embryonic precursors like vascular and visceral SMCs could acquire a distinct chromatin structure associated with expression of genes for example needed to perform different types of contraction.

Only recently, studies on the role of chromatin restructuring in SMC-specific gene expression have gained momentum. Data on the SM-MHC CArG boxes support a model in which SMC-restricted genes are in an open chromatin structure in SMCs, whereas in other cells this region of the chromatin is not accessible ${ }^{258}$. It has also been shown that histone hyperacetylation contributes to the regulation of many $S M C$ genes via SRF in the early stages of SMC differentiation ${ }^{195}$. It was reported that SRF levels in A404 cells did not change after differentiation towards SMCs, yet SRF was only able to bind CArG boxes in the differentiated cells, which contained hyperacetylated $\mathrm{H} 3$ and $\mathrm{H} 4$ histones. Interestingly, the histones of different CArGcontaining regions of the SM-MHC and $\alpha$-SMA genes were acetylated to a different extent.

Specific chromatin-modifying histone-acetyltransferases (HAT) that have been shown to regulate SM22 $\alpha$ expression include CREB-binding protein (CBP) and PCAF (p300/CBP-associated factor). CBP is an SRF-coactivator with HAT activity which was recruited to the CArG box region of the SM22 $\alpha$ promoter, and increased its activity ${ }^{273}$. PCAF increased promoter activity as well. SM22 $\alpha$ promoter activity was attenuated by overexpression of specific histone-deacetylases (HDAC). Thus, SRF interacts with a protein or protein complex with HAT activity. The effects of HATs and HDACs on SMC-specific gene transcription may be modulated by $Y Y 1$, since this transcription factor has been shown to interact with CBP and $p 300^{290,291}$. YY1 can 
either activate or repress transcription via interactions with these chromatin structure modifying enzymes ${ }^{292}$. The effects of $Y Y 1$ on SMC promoter activity have only been investigated for $S M 22 \alpha$, which is activated ${ }^{253}$. Besides modification of histones, DNA-modifications can also affect promoter activity. Only one study on $S M C$ gene regulation addressed this issue, showing that human $S M 22 \alpha$ promoter activity is decreased by methylation of CPG dinucleotides ${ }^{67}$.

It is now obvious that mechanisms of chromatin remodeling that modulate the CArG box/SRF transcriptional network are operational in SMCs. Future studies in this area will likely reveal additional histone-modifying enzymes involved in the regulation of expression of genes specifically in SMCs, thereby co-determining SMC phenotype.

\section{Additional transcriptional pathways regulating smooth muscle cell gene expression}

The highly divergent environments in which SMCs reside surrounds them with different stimuli. This will probably necessitate the use of different combinations of regulatory modules in different subsets of SMCs, even for expression of the same gene. Studies in transgenic mice have indeed revealed that additional regulatory elements besides the CArG box are required for correct recapitulation of the endogenous expression profile of SMC-restricted genes. Besides these elements, several other motifs have been found to regulate SMC-restricted genes in cultured SMCs, but have not been tested in vivo. These will also be discussed in this section.

\section{TGF- $\beta$ : TGF- $\beta$ control element, GKLF \& BTEB2}

As outlined before, TGF- $\beta$ regulates the SMC phenotype in many ways. Some of the effects of TGF- $\beta$ ultimately converge at a so-called TGF- $\beta$ control element (TCE; core sequence 'GAGTGGG') in the promoters of SMC-restricted genes. The functionality of this element in vitro was first revealed for the $\alpha$-SMA promoter ${ }^{186}$. Later, it was shown that it is a critical element for SM22 $\alpha$ promoter activity in vivo, for its mutation caused a complete loss of activity ${ }^{293}$. The TCE can bind different factors that are regulated by TGF- $\beta$ : GKLF (gut-enriched Krüppel like factor), or BTEB2 (basic transcriptional element binding protein-2). These proteins have opposite effects on transcriptional activity. GKLF has been shown to repress TGF- $\beta$-induced transcription whereas BTEB2 activated transcriptional activity of the SM $22 \alpha$ gene $^{293}$. Hence, TGF- $\beta$ can exert both stimulatory and inhibitory effects on SMC marker gene expression. GKLF expression in contractile SMCs however is low, and the high GKLF-levels in cultured SMCS are downregulated by TGF- $\beta$ treatment. Since GKLF is expressed in many non-SMCs, it may repress transcription of SMCrestricted genes in non-SMCs. This activity is probably mediated by bone morphogenetic proteins, which are members of the TGF- $\beta$ superfamily that regulate GKLF-binding to the TCE ${ }^{294}$. The stimulatory TGF- $\beta$ regulated factor, BTEB2, 


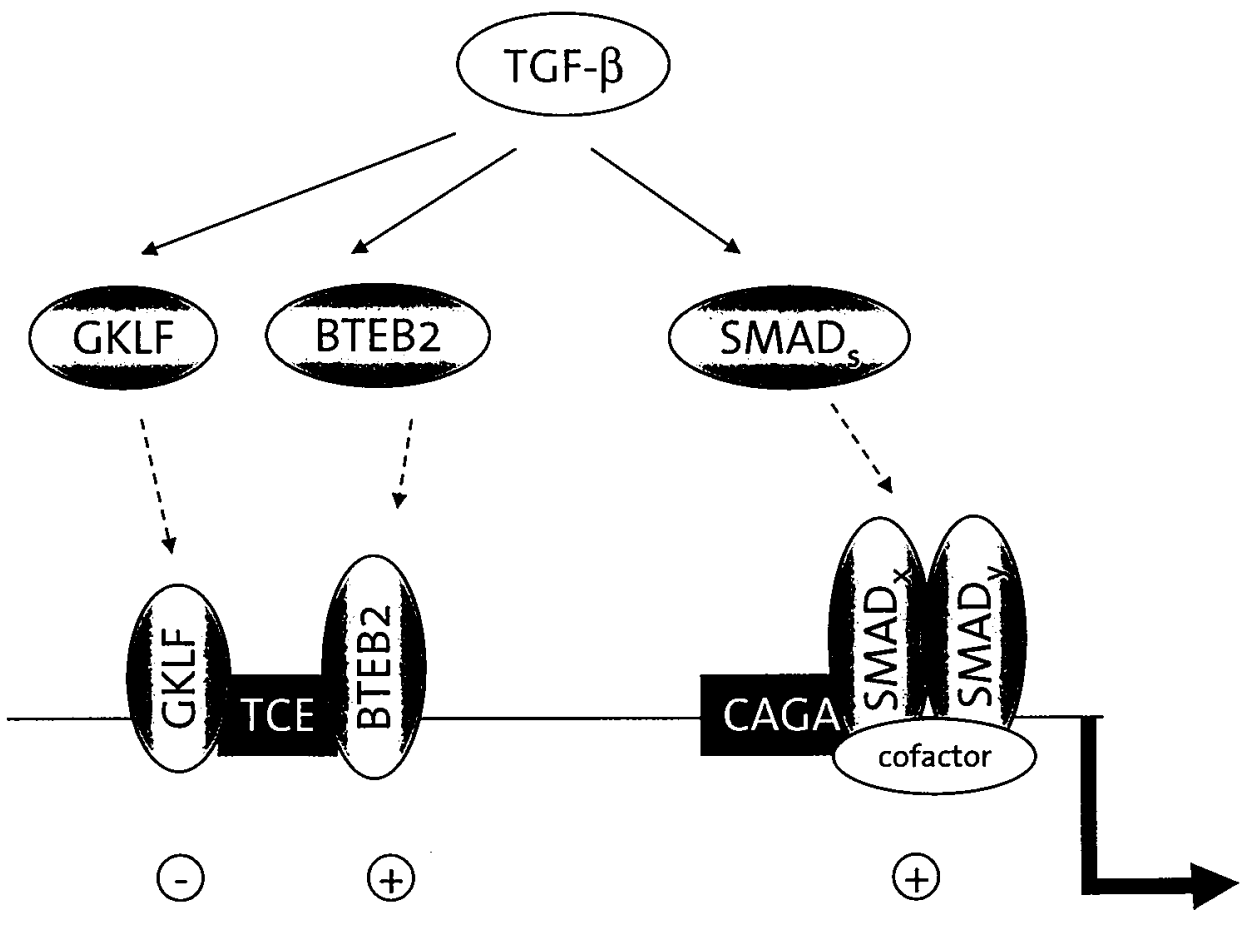

Figure 1.9 Pathways of TGF- $\beta$-mediated effects on SMC gene transcription

is abundantly expressed in smooth muscle tissues, and has been shown to increase promoter activity of SMemb ${ }^{295}$. It is upregulated in synthetic SMCs in vitro and in vivo, activating expression of not only SMemb but also several other genes associated with the synthetic phenotype of SMCs ${ }^{296}$. BTEB2 interacts with the RAR, indicating a pathway through which retinoids may influence $S M C$ phenotype. Knocking out the BTEB2 gene in mice is embryonically lethal ${ }^{297}$.

TGF- $\beta$ : CACA-box \& SMADs

The effects of TGF- $\beta$ on SMCs are not exclusively mediated by the TCE. A second TGF- $\beta$ regulated element in promoters of both synthetic and contractile SMC genes is the CAGA-box. This motif, which is for example present in the SM22 $\alpha$ and the CARP promoter, has been shown to bind SMADs ${ }^{190,298}$. The CAGA-box in the SM22 $\alpha$ promoter acts in concert with a so-called medea box which was shown to be necessary for formation of the TGF- $\beta$-inducible complex and for promoter activity $^{298}$. Interestingly, the functional SM22 $\alpha$ CAGA motif is located in the $5^{\prime}$ untranslated region of its first exon. Similarly, $\alpha-S M A, S M-M H C$, and SM-calponin contain putative SMAD binding motifs in their $5^{\prime} U T R^{298}$. The collagen type 1 promoter also contains a TGF- $\beta$-responsive region with binding sites for SMADs ${ }^{189}$, and the promoters of several other collagen isoforms and $\beta 5$ integrin are activated by SMAD proteins via CAGA and medea motifs ${ }^{299}$. 
Thus, TGF- $\beta$ effects on SMC phenotype are ultimately caused by transcriptional regulation of both contractile and synthetic target genes via multiple trans-acting factors and cis-acting elements in their promoters (figure 1.9). This complex way of regulation by both positive and negative factors has probably evolved to provide the SMC with the flexibility to rapidly change expression levels of genes critical for its function in different situations.

\section{E-boxes \& basic-Helix-Loop-Helix factors}

E-boxes are CANNTG sequences which can be found scattered throughout SMCspecific promoters. These elements are bound by homo- or heterodimers of class I and class II basic-Helix-Loop-Helix (bHLH) transcription factors. Class I bHLH proteins are ubiquitously expressed, but a cell type specific complex is formed by dimerization with the tissue specific class $\| \mathrm{bHLH}$ proteins. Furthermore, Id and Twist bHLH proteins inhibit gene regulation by bHLH complexes through formation of non-functional heterodimers ${ }^{300}$. E-boxes have been found to regulate expression of APEG-1 and osteopontin ${ }^{90,301}$. They are critical for $\alpha$-SMA expression in vivo ${ }^{262}$. bHLH proteins that activated $\alpha$-SMA transcription in SMCs include the class 1 factors E12 and USF (upstream stimulatory factor) proteins ${ }^{302}$. Class II bHLH factors like dHAND, eHAND, and capsulin were not able to activate the $\alpha$-SMA promoter, and Id and Twist attenuated activity. Interestingly, SRF and several bHLH factors were found to activate the $\alpha$-SMA promoter synergistically through a mechanism distinct from direct interaction ${ }^{262}$. This suggests that besides direct interactions between SRF and SRF-cofactors, additional mechanisms exist to mediate SRFeffects on SMC gene expression.

What emerges from these results is that bHLH factors and interactions between bHLH factors and SRF play an important role in controlling expression of some SMC-restricted genes. More studies employing the promoters of other SMCrestricted genes are needed to reveal if this is an additional general mechanism by which SMC marker genes are transcriptionally regulated.

\section{MEF2 sites and proteins}

Myocyte enhancer binding factor-2 (MEF2) proteins homo- or heterodimerize to bind an A/T-rich cis-element that is present in many SMC-specific promoters. Several MEF2 family members are associated with the synthetic phenotype of SMCs. MEF2A, MEF2B, and MEF2D are expressed in proliferating synthetic SMCs, but not in contractile $S M C s^{303}$. In addition, MEF2C protein, but not mRNA, is increased in neointimal SMCs. Post-transcriptional regulation of MEF2C in SMCs may thus be of importance for SMC phenotype. Even though MEF2C levels are higher in synthetic SMCs, MEF2C is important for contractile gene expression as revealed by MEF $2 C$ knockout mice. These mice have a silent SM22 $\alpha$ promoter and defective contractile SMC development ${ }^{304}$. Interestingly, there is no essential MEF2 binding site in the $S M 22 \alpha$ promoter. This suggests that like the other factors regulating SMC differentiation, MEF $2 C$ cooperates with other transcription factors. 
Of note, like many SMC marker genes, heterogeneous expression of MEF 2 isoforms in SMC subsets has been observed ${ }^{303}$. Consequently, this may be one of the factors involved in specifying SMC heterogeneity.

\section{Gax}

Gax (growth-arrest-specific homeobox) is a homeodomain transcription factor with high expression levels in contractile SMCs and low levels in cultured SMCs or proliferating SMCs after balloon injury ${ }^{305,306}$. Overexpression of Gax inhibits SMC proliferation and migration, presumably via targeting of p21 and downregulation of $\alpha v \beta 3$ and $\alpha v \beta 5$ integrins ${ }^{307,308}$. The reduction of proliferation has been shown to be essential but not sufficient for the antimigratory effects of Gax. The Gax promoter is activated by both Sp1 and MEF2, but the tissue-specific and mitogeninhibited expression of Gax was not conferred by these factors ${ }^{309}$. Future studies are required to elucidate the possible role of Gax in transcriptional regulation of genes in contractile SMC and to identify cis-elements that mediate these putative effects.

\section{GATA elements and GATA- 6}

GATA- 6 is a zinc finger transcription factor that is expressed in a temporal pattern reminiscent of that of several SMC-restricted genes. It has high expression levels in vascular SMCs, bladder, and lung, but cannot be detected in the uterus or gastrointestinal system ${ }^{310}$. GATA- 6 expression is associated with the contractile SMC phenotype, as evidenced by its downregulation in proliferating SMCs and its ability to induce cell cycle arrest after overexpression ${ }^{311,312}$. Like Gax, the effects of GATA-6 on SMC proliferation seem to be mediated by the cell cycle regulator $p 21$ and are thought to operate at the posttranscriptional level. GATA-6 binding to GATA elements, which are present in many SMC promoters, has been shown to augment SM-MHC promoter activity ${ }^{313}$. GATA- 6 interacts with the transcriptional coactivator p300, and its DNA binding activity is enhanced by PPAR $\gamma$ ligands ${ }^{314}$. A second way of activating GATA- 6 is by interaction with nuclear factor of activated $T$ cell $c 1$ (NFATC1), a calcineurin-activated transcription factor. GATA-6 and NFATC1 have been shown to synergistically activate the SM-MHC promoter, while blocking NFATC1 inhibited SM-MHC and $\alpha$-SMA expression ${ }^{315}$. Calcineurin inhibitors have been shown to drive $S M C$ modulation towards the synthetic phenotype ${ }^{316}$. Because calcineurin is activated by $\mathrm{Ca}^{2+}$-dependent phosphatases ${ }^{317}$, these results have led to the suggestion that $\mathrm{Ca}^{2+}$ may be involved in SMC differentiation, like in skeletal muscle. Interestingly, NFATC1 can interact with p300, suggesting that a GATA6/P300/NFATC1 complex may be formed in SMCs to activate SMC-specific target genes.

\section{$S p 1 \& A P-1$}

Sp1 and activator protein-1 (AP-1) are involved in the activation of many genes in diverse cell types, including SMCs. It is known that Sp1 can interact with SRF to 
regulate genes in other muscle cells ${ }^{318}$. By analogy, a distinct SMC-specific Sp1coactivator complex may exist. The present data however indicate that neither Sp1 nor AP-1 confers SMC-specificity or phenotype-specificity of expression. Instead, they have been found to increase expression of both contractile and synthetic SMC genes. Sp1 has been shown to activate SM-MHC, ACLP, elastin, and collagen type I promoters ${ }^{96,319-321}$. AP-1 activates tissue inhibitor of metalloproteinase-1 (TIMP-1) and MMP-1 expression ${ }^{322}$. Although elastin gene transcription is repressed by an AP-1 element in its promoter ${ }^{323}$, it appears that the overall effects of the AP-1 complex lead to increased SMC proliferation and migration ${ }^{324}$.

\section{$M r f 2 \alpha \& M r f 2 \beta$}

The A/T-rich interaction domain transcription factors $M r r_{2} \alpha$ and $M r f 2 \beta$ were identified by a differential display screen of Monc-1 cells that were induced to differentiate towards SMCs ${ }^{325}$. They were found to increase $\alpha$-SMA and SM22 $\alpha$ levels, and to inhibit cell proliferation. Late differentiation markers like SMcalponin, SM-MHC, and smoothelin were not increased by the Mrf2s. Like the other transcription factors regulating SMC differentiation, Mrf $2 \alpha$ and Mrf $2 \beta$ are not exclusively expressed in SMCs. Mrf $2 \alpha$ seems to be the dominant isoform in SMCs because of its absence in Monc-1 cells that differentiated towards non-SMCs and its presence in lung, aorta and heart. Of note, CArG boxes are able to compete for Mrf2s in gel shift assays, indicating that Mrf2s may also bind to the CArG element. The domains that are present in the Mrf2 isoforms also indicate that they may act by remodeling chromatin structure ${ }^{325}$.

\section{Hepatocyte nuclear factor-3 homologue 1}

Negatively acting elements in promoters of SMC-restricted genes can serve to inhibit expression of these genes in non-SMCs. Thus far, only one factor has been identified that acts in this way, hepatocyte nuclear factor-3 homologue $1(\mathrm{HFH}-1)$. $\mathrm{HFH}-1$ binds to an A/T-rich element in SMC promoters and is readily detected in epithelial cells of the stomach and the bladder, in contrast to SMCs, which contain only low amounts ${ }^{326}$. Telokin, $\alpha$-SMA, SM-MHC, and SM22 $\alpha$ expression have been shown to be inhibited by $\mathrm{HFH}-1$, presumably by blocking the interaction of activating factors with the A/T-rich element. However, MEF2 competition for the A/T-rich element in the telokin promoter did not affect promoter activity ${ }^{87}$. In addition, $\mathrm{HFH}-1$ did not affect SRF-binding to CArG boxes.

It is plausible to suspect that there are more factors in non-SMCs that repress transcription of SMC-restricted genes. In fact, these may be just as important for restricting expression as transcription enhancing factors in SMCs. That only few such factors have been identified is related to the fact that most studies on SMC transcription regulation have used SMCs in their experiments. Future studies using SMC-specific promoters in non-SMCs and nuclear extracts from non-SMCs in gelshift assays will probably identify more factors important for repression of SMCspecific genes in non-SMCs. 


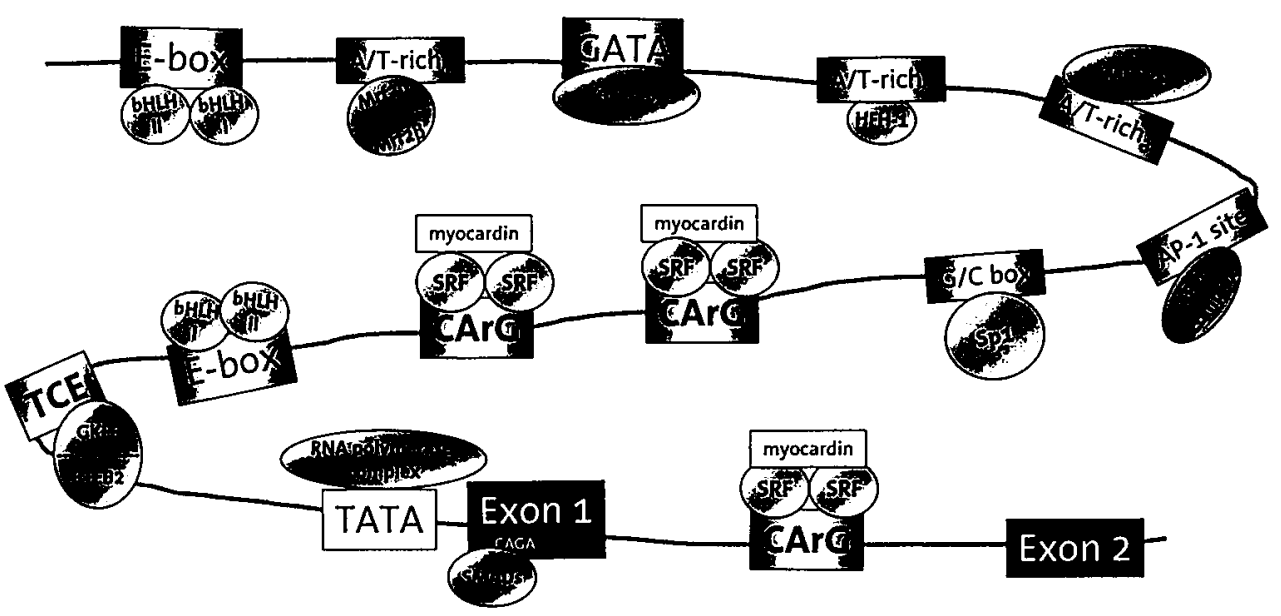

Figure 1.10 Hypothetical SMC-specific promoter containing most described cis-elements involved in regulation of SMC-restricted gene expression. The indicated A/T-rich sequences are not necessarily the same.

On the whole, it is evident that many different cis-elements and trans-factors do not only contribute to, but are critical for the regulation of SMC genes. What also can be distilled from the data is that virtually all factors that regulate SMC gene transcription interact with at least one other factor. In addition, it appears that many cis-elements in SMC promoters are multifunctional, i.e. they can interact with different trans-factors, causing distinct effects on gene expression. As more and more transcription (co)factors and the interactions between them will be identified in the future, it may be possible to construct a general building plan for a SMC-specific promoter. In figure 1.10, such a building plan based on today's knowledge is presented. It is likely that many gaps are present in this schematic representation, and it is clear that not all SMC promoters conform to this configuration. Yet, it would be interesting to construct a synthetic promoter according to this plan, and evaluate its activity and expression pattern.

\section{Putative components of the SMC transcriptional program}

The cis-elements and trans-factors discussed so far have been shown to regulate promoter activity of several SMC genes. Consequently, it is likely that they are components of the general transcriptional program for either a contractile or a synthetic SMC. Many additional elements/factors have been found to regulate expression of one particular SMC gene. Since most of these elements have only recently been described, their contribution to the SMC's transcriptional regulation in general is not established at present. In this section, some of those recently reported regulators are reviewed. 
Putative components of the contractile transcriptional program

Many putative components of the contractile transcriptional program have been identified by using the $\alpha$-SMA promoter. This is a consequence of its high transcriptional activity in contractile SMCs, making $\alpha$-SMA the most extensively studied SMC-restricted gene. Besides CArG boxes, E-boxes, and a TCE, $\alpha$-SMA promoter activity is regulated by the homeoprotein Mhox, which has been shown to increase SRF-binding to one of the CArC boxes ${ }^{186,285,327}$. Because SRF-dependent regulation of SMC genes is so conserved, it is likely that promoter activity of other SMC genes is affected by Mhox as well. Aside from Mhox, $\alpha$-SMA promoter activity is also controlled by so-called MCAT elements. These bind transcription enhancer factor 1 (TEF-1) transcription factors, causing activation of gene transcription. Interestingly, the opposite strands of MCAT elements interact with the singlestranded DNA (ssDNA)-binding proteins Pur $\alpha$, Pur $\beta$, and MSY1, which repress transcription. In addition, Pur $\alpha, P u r \beta$, and MSY1 interact with each other ${ }^{328}$. It has been suggested that TEF- 1 factors can only activate transcription after dissociation of the ssDNA-binding proteins ${ }^{329}$. The TEF- 1 members and Pur $\alpha, P u r \beta$, and MSY 1 can interact with SRF, increasing the possibility that they are also functional in the regulation of other SMC genes. Moreover, these ssDNA-binding proteins seem to be involved in posttranscriptional regulation of $\alpha$-SMA expression via interaction with RNA containing an MCAT-like element in exon 3 of the $\alpha$-SMA gene ${ }^{330}$. Squelching of Pur $\alpha$, Pur $\beta$, and MSY1 to newly transcribed mRNA may attenuate their repressive effects on transcription. Alternatively, they may have effects on the regulation of pre-mRNA splicing.

Additional regulators of the contractile SMC phenotype may be identified by studies on the SM-MHC and smoothelin promoters, since these are the proteins that are most specific for the contractile SMC phenotype. In addition, SM-MHC appears to be the only SMC marker gene that is exclusively expressed in SMCS during development and adult life. Consequently, this promoter offers a possibility to identify transcriptional elements that direct expression exclusively in SMCs throughout development, if these exist.

\section{Putative components of the synthetic transcriptional program}

The regulation of genes which expression goes up with modulation towards the synthetic SMC type is not dominated by one cis-element like the CArG box for contractile marker genes. Instead, many different cis-elements have been reported to regulate expression of those genes. It may be that this is a prerequisite for balanced expression, providing the possibility of quick responses to changed conditions that require alterations in gene expression. Alternatively, the 'master' factor inducing expression of synthetic genes may not have been identified. A candidate factor is the homeobox protein Hex, which regulates promoter activity of SMemb ${ }^{32}$. Hex is induced in cultured SMCs and neointimal SMCs in vivo and follows the same spatiotemporal expression pattern as the SMemb gene. In the SMemb promoter, it acts through a cyclic adenosine monophosphate (CAMP) 
responsive element (CRE). Although its transactivation potential is enhanced by the CRE binding (CREB) protein, Hex and CREB do not directly interact. In contrast to Hex, members of the hcKrox transcription factor family have been found to repress transcription of several ECM genes, among which collagen type l, fibronectin and elastin $^{322}$. As such, they appear to be components of a negative transcriptional program for a synthetic SMC.

\section{Posttranscriptional control of SMC differentiation markers}

Regulation of SMC phenotype occurs not only at the transcriptional level, but also posttranscriptionally. For instance, selection of SMC-specific exons of $\alpha$ tropomyosin, h-caldesmon, meta-vinculin, and $\alpha$-actinin is downregulated when SMCs modulate towards the synthetic phenotype ${ }^{42}$. The mechanisms responsible for SMC-specific splicing have been investigated with the help of these genes.

Tissue-specific alternative splicing is determined by cis-acting elements, including specific branch point sequences, polypyrimidine tracts, and splicing enhancers, and trans-acting factors of which the nature is largely unknown. However, some of the determinants of SMC-specific splicing, and even of differential splicing between SMC subsets have been identified. For example, three distinct splicing enhancers and two silencers have been shown to be involved in the determination of alternative splicing of the SM myosin phosphatase subunit 1 (MYPT1) ${ }^{331}$, and repeating purine-rich motifs mediate caldesmon mRNA splicing ${ }^{42}$. SMC-specific splicing of $\alpha$-tropomyosin and $\alpha$-actinin is regulated by the polypyrimidine tract binding protein (PTBP). This is a splicing repressor expressed in many cell types which mechanism of action is unclear. It has been suggested to act by sequestering or coating exons, thereby preventing splicing ${ }^{332}$. Both $\alpha$-tropomyosin and $\alpha$-actinin contain a SMC-specific exon and an exon which is only present in mRNA of other cell types ${ }^{333}$. Interestingly, both repression of the SMC-specific $\alpha$-actinin exon in non-muscle cells as well as SMC-specific inhibition of the default non-muscle exon 3 of $\alpha$-tropomyosin has been shown to be regulated by PTBP ${ }^{334.335}$. In the case of the $\alpha$-actinin gene, PTBP acts in conjunction with regulatory elements between the branch point and the $3^{\prime}$ splice site of the SMC-specific exon ${ }^{335}$. Repression of $\alpha$ tropomyosin is mediated by three negatively acting cis-elements in its pre$\mathrm{mRNA}^{336}$. Because of its ubiquitous expression, polypyrimidine tract-binding protein is probably not responsible for SMC-specific splicing by itself, but instead, may require additional factors which could be tissue-restricted. Three CELF family proteins have been tested for this activity, but were not able to confer SMC-specific splicing to the $\alpha$-tropomyosin gene ${ }^{336}$. Other functional splicing regulators in SMCs are the Bruno-like proteins Bru1 and Etr-3, which bind to repeat elements of uridine and purine in $\alpha$-actinin pre-mRNA ${ }^{337}$.

Although identification of the regulators of SMC splicing is difficult, it is likely that 
alternative splicing of caldesmon and vinculin is mediated by a mechanism distinct from that used for $\alpha$-actinin and $\alpha$-tropomyosin. This notion comes from a study directed towards the identification of SMC-specific regulators of splicing, employing a splicing-dependent selection system. It revealed co-regulation of $\alpha$ actinin and $\alpha$-tropomyosin splicing, but at the same time no SMC-specific splicing of caldesmon and vinculin ${ }^{333}$.

A splicing element which is only functional in SMCs has not been identified so far. The current point of view therefore is that there are no dedicated SMC-specific regulators but rather that SMC-specific splicing is fine-tuned using a particular arrangement of cis-elements. The functional strength of these elements is determined by variations in the activities and levels of various factors, among which PTBP, making sure that appropriate exon skipping or inclusion is most manifest in SMCs.

\section{Determination of smooth muscle cell phenotype and differentiation: a model}

\section{Regulation of SMC-specific transcription}

Three levels of regulation of SMC marker gene expression have been discussed: regulation at the chromatin level by histone modifying enzymes, at the DNA level by cis-elements and transcription factors, and at the pre-mRNA level by splicing regulators. The importance of regulatory mechanisms at all these levels for SMC differentiation and phenotypic modulation is undeniable.

The highest order of regulation, i.e. chromatin conformation, has a great potential to restrict expression of genes. This type of regulation can overrule the other mechanisms and thereby determine the activity of a gene in a SMC. It is now known that genes that are actively transcribed in SMCs are located in regions of open chromatin ${ }^{195,273}$. Exclusion of transcription of these genes in non-SMCs can simply be achieved by keeping the cis-elements inaccessible for the activating transcription factors. This may also be accomplished by methylation of $\mathrm{CPC}$ doublets in promoters of SMC-restricted genes ${ }^{67}$.

The studies on transcriptional regulation of SMC marker genes have revealed that although the promoters of these genes appear highly disparate, they still have many cis-elements in common. Most of these elements interact with more or less ubiquitously expressed transcription factors. This would imply that widely expressed factors keep expression of SMC marker genes restricted to SMCs. As stated above, regulation of SMC-specific expression by chromatin conformation can explain how these ubiquitously expressed transcription factors can still regulate expression of genes specifically in SMCs. In addition, it is now recognized that although these transcription factors are expressed in several cell lineages, they may form a specific combination only in SMCs. These SMC-specific complexes do 
not consist of DNA-binding transcription factors only. Instead, cofactors interacting with the transcription factors may play a more important role. These cofactors position the complex at the proper site to initiate transcription, simplify interaction with other complexes, and induce conformational changes which activate or repress transcription ${ }^{282}$.

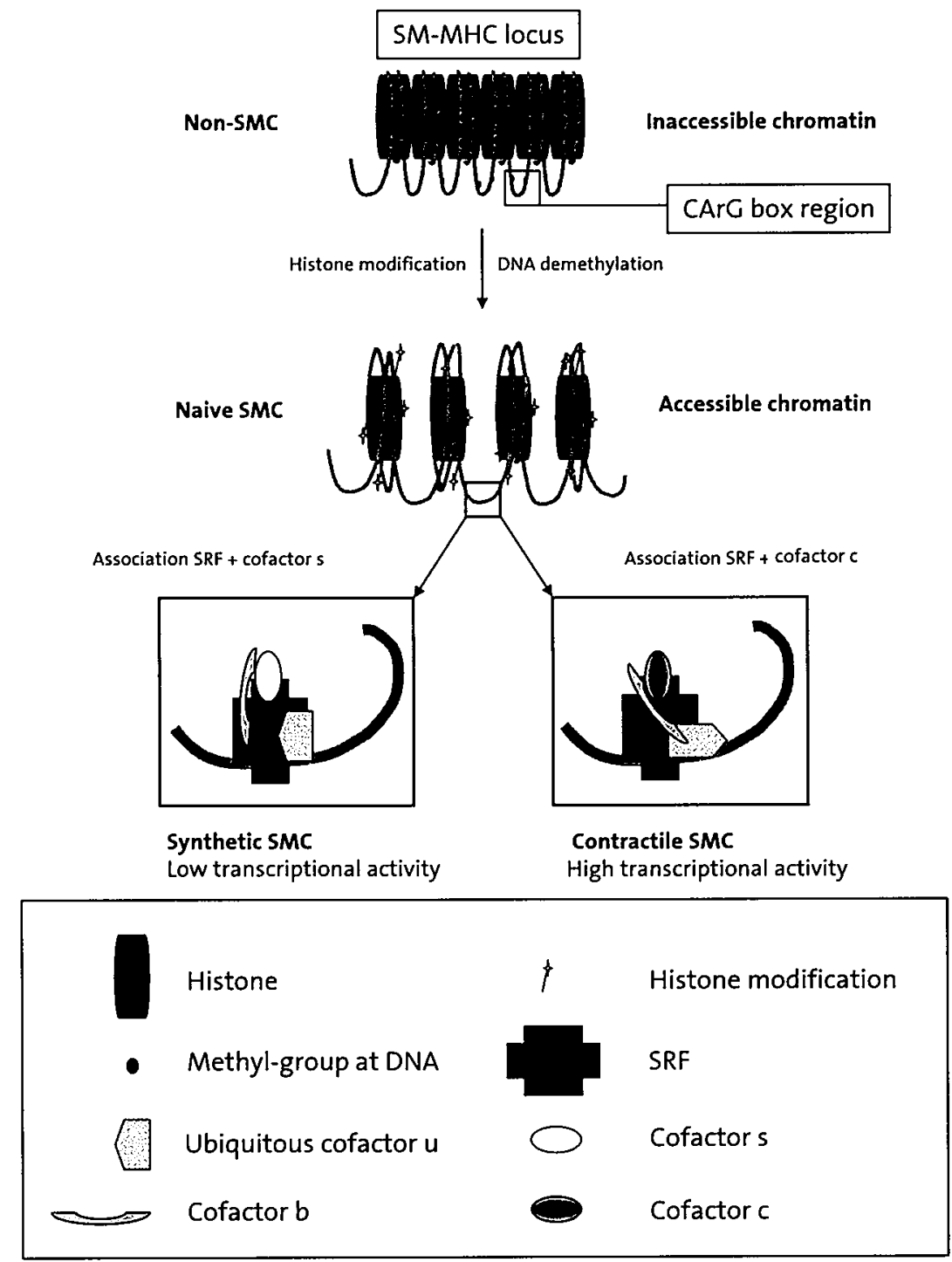

Figure 1.11 A model of contractile gene transcription regulation in SMCs. The SM-MHC gene is taken as an example. Its locus is not accessible for transcription factors in non-SMCs because of its chromatin conformation. After histone modification and DNA demethylation, SRF can interact with the CArG boxes in the SM-MHC promoter. Because of interaction with different cofactors, SRF directs either low- or high-level transcription of SM-MHC, which is associated with the SMC phenotype. 


\section{Regulation of SMC phenotype-specific transcription}

The regulation of SMC marker genes runs parallel with the regulation of SMC phenotype, for the phenotype is the sum of the expression level of many genes, all regulated by trans-activating complexes. The composition and stability of these complexes is influenced by 1) the local environment consisting of cytokines, growth factors, ECM components, and mechanical factors, and 2) the origin and developmental stage of the cell, which both affect the abundancy of particular trans-factors. Differences in complex-structure will be translated into SMC gene expression profiles and thus into SMC phenotype, resulting in SMC heterogeneity. This heterogeneity of SMCs and the phenomenon of SMC phenotypic modulation makes it unlikely that a general program explaining all the phenotypes that a SMC can adopt exists. Rather, we suggest that a basal differentiation program, presumably partly built on SRF-CArG box interactions, leads to the development of a 'naive SMC'. This naive SMC, with open chromatin at SMC gene loci, expresses both contractile and ECM proteins at a considerable level. Fine-tuning of the function of the naive SMC according to the demand of the local environment then ultimately defines its precise characteristics. These characteristics are determined by the levels of proteins that are associated with either the contractile or the synthetic phenotype. It is likely that the fine-tuning of the SMC phenotype involves continuous regulation. This may be accomplished by a combination of positive- and negative-acting factors, or by association of specific cofactors with an integrating protein like SRF.

In our model (figure 1.11), the precise phenotype of a SMC is the outcome of the balance between (co)factors that promote the contractile phenotype and (co)factors that activate the synthetic expression program. The composition of the transactivation complexes, either distinct because of incorporation of a unique (co)factor or because of different concentrations of a common (co)factor determines the transcriptional activity of the gene. Rapid changes in the levels of proteins associated with a specific phenotype can be brought about by specific posttranslational modifications which disrupt or stabilize transactivational complexes. It is important to note that the transcription factor complexes can interact with chromatin remodeling factors. This allows initially transient changes in complex composition to be translated into more persistent alterations of gene expression.

Posttranscriptional regulation represents an additional level of regulation which provides more diversity in gene transcripts. Many SMC markers are alternatively spliced isoforms of more widely expressed genes. Currently, not much is known about this kind of regulation, although it is clear that it has great effects on SMC phenotype. However, the current great interest in the study of SMC differentiation and phenotypic modulation will accelerate our understanding of the underlying regulatory mechanisms in this versatile cell type. 


\section{Smooth muscle cell function: the dual role of the SMC}

The principal function of SMCs in healthy adult tissues is contraction. Apart from this, they also generate ECM components to secure the structural integrity of the tissues they are part of. Under certain physiological conditions and in diseased smooth muscle tissues, this second function of the SMC becomes more important. SMCs may contribute to remodeling and repair of smooth muscle tissues by increased proliferation and synthesis of more or different ECM components.

\section{Smooth muscle contraction and relaxation}

\section{Smooth muscle contraction versus skeletal muscle contraction}

Smooth muscle contraction differs profoundly from contraction in skeletal muscle, even though it is based on the same basic mechanism of myosin and actin filament interaction, activated by high $\mathrm{Ca}^{2+}$-levels. This is partly related to the fact that SMCs have their own unique contractile protein isoforms ( $\alpha-S M A$ and $\gamma$-SMA, and SMMHC isoforms), which are of great importance for the characteristics of SMC contraction as evidenced by the phenotype of $\alpha$-SMA null mice. In these mice, $\alpha$ SMA is replaced by skeletal $\alpha$-actin, leading to impaired vascular contractility and tone, and reduced blood flow ${ }^{60}$. The different SM-MHC variants in SMCs are also associated with specific SMC contractile characteristics. The SM-A isoforms that vary in the $C$-terminal region influence the organization of the myosin filaments ${ }^{338}$. The SM-B isoforms that include seven amino acids extra in the $\mathrm{N}$-terminal head region are associated with an increased shortening velocity ${ }^{65,339}$.

Besides the use of SMC-specific isoforms of myosin and actin, two major differences between skeletal and smooth muscle are apparent: the spatial organization of the myosin and actin filaments, and the regulation of the contraction, which will be extensively discussed below. Different from the highly structured sarcomeres in skeletal muscle cells, the actin filaments of SMCs do not display a strict organization, but radiate from dense bodies into all directions ${ }^{8}$ (figure 1.1). Interspersed among the actin filaments and in between two dense bodies, myosin filaments are present, which are shorter than and far outnumbered by the actin filaments. Although SMCs contain only one-fifth of the myosin of skeletal muscle, their myosin filaments are longer, partly explaining the greater force that SMCs can generate compared to skeletal muscle cells ${ }^{340}$.

As compared to skeletal muscle, SMC contraction is slower, but more powerful and persistent, yet less energy consuming. The following properties of the SMC contractile apparatus largely explain the different SMC contraction profile. First, the cross-bridge cycling rate of the myosin filaments is much slower in SMCs. This is partly related to the low ATPase activity of the SM myosin heads. Also, action potentials in SMCs are generated by $\mathrm{Ca}^{2+}$ via $\mathrm{Ca}^{2+}$-channels, which are considerably slower than the $\mathrm{Na}^{+}$-channels used in skeletal muscle cells. Thus, the rapidity of 
contraction is diminished. Second, because the time of cross-bridge interaction is longer in SMCs, the force of contraction is much higher. In addition, SM myosin can generate four times greater force per cross-bridge head than skeletal muscle myosin $^{341}$. Third, the slow detachment of the cross-bridges and the slowness of the pumps that remove $\mathrm{Ca}^{2+}$ out of the SMCs cause a longer persisting contraction. Finally, SMCs can shorten much more than skeletal muscle cells, which is probably related to the longer actin filaments and their different organization. Because of the absence of sarcomeres, SMC myofilaments have more freedom of movement and consequently can contract a SMC to shorter lengths.

\section{Smooth muscle contraction regulation by myosin phosporylation}

Some important activators of SMC contraction are autonomic nerves that secrete norepinephrine, hormones like angiotensin and vasopressin, changes in $\mathrm{O}_{2}-$ and $\mathrm{CO}_{2}$-concentration, and stretch ${ }^{342}$. These stimuli cause depolarization of the membrane, thereby initiating an increase of $\mathrm{Ca}^{2+}$-levels which induces contraction. $\mathrm{Ca}^{2+}$ can enter the cytoplasm from the extracellular space by various $\mathrm{Ca}^{2+}$-channels and other ion channels that are permeable to $\mathrm{Ca}^{2+}$. A second way of elevating $\mathrm{Ca}^{2+}$ concentration is by release from the intracellular SR through ryanodine and inositol triphosphate receptors ${ }^{343}$. This last mechanism is less important for generating SMC contraction than for generating skeletal muscle contraction. In SMCs, most of the $\mathrm{Ca}^{2+}$ enters from the extracellular space and diffuses throughout the cell. The local $\mathrm{Ca}^{2+}$-concentration is determined by the entry points and the distribution of intracellular $\mathrm{Ca}^{2+}$-binding molecules and transporters which extrude the $\mathrm{Ca}^{2+}$-ions. Together with the specific allocation of the effector molecules, this allows temporospatial defined responses to $\mathrm{Ca}^{2+}$-levels ${ }^{344}$.

$\mathrm{Ca}^{2+}$-induced activation of SMC contraction is mediated by many proteins (figure 1.12). For activation of myosin in the contractile machinery, an increase of $\mathrm{Ca}^{2+}$. levels throughout the cell for a sufficient time to occupy the $\mathrm{Ca}^{2+}$-binding sites of calmodulin is necessary. Calmodulin subsequently activates MLC kinase by association with its catalytic subunit, which phosphorylates the regulatory $\mathrm{MLC}_{20}$. After phosphorylation of $\mathrm{MLC}_{20}$, assembly of myosin filaments from myosin monomers is facilitated. More importantly, myosin ATPase activity is increased and thus the myosin head is able to bind to the actin filament, thereby generating contraction. Contraction stops once MLC phosphatase dephosphorylates the $\mathrm{MLC}_{20}{ }^{345}$. Hence, the level of $M \mathrm{MC}_{20}$ phosphorylation and the resulting force production is the outcome of the balance between MLC kinase and MLC phosphatase activity. MLC kinase and MLC phosphatase activity are also regulated in a $\mathrm{Ca}^{2+}$-independent way, thereby affecting the $\mathrm{Ca}^{2+}$-sensitivity of SMC contraction. Especially $\mathrm{Ca}^{2+}$-independent regulation of $\mathrm{MLC}$ phosphatase activity through its regulatory subunit MYPT1 is important for SMC contraction dynamics. MLC phosphatase activity is upregulated by cyclic guanosine monophosphate (CGMP)- and possibly cyclic adenosine monophosphate (CAMP)-dependent protein kinases (PKG \& PKA), and downregulated by $\mathrm{CPI}-17$ and by phosphorylation 


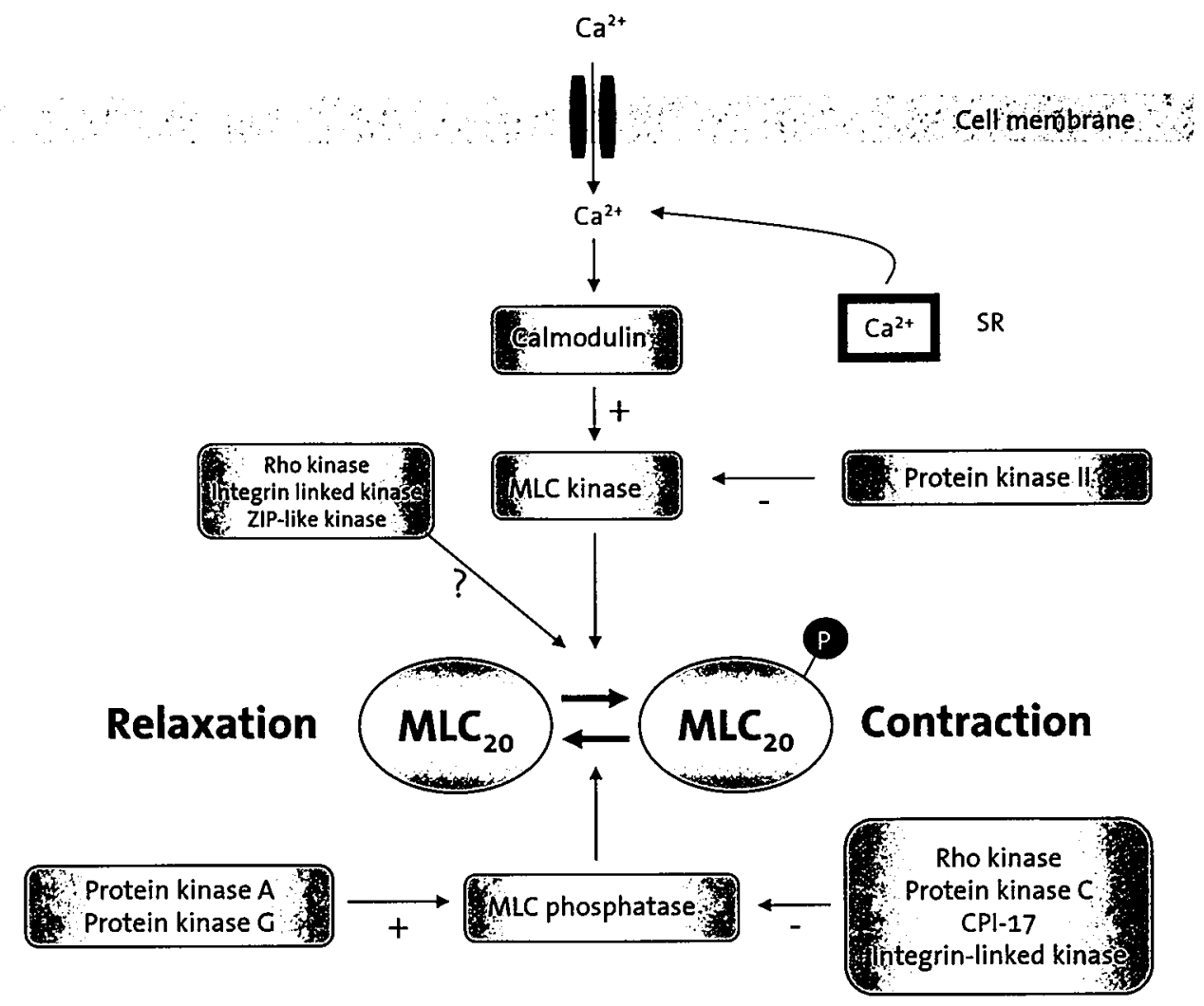

Figure 1.12 SMC contraction regulation. Scheme of the diverse pathways of $M L C_{20}$ regulation.

through Rho kinase, integrin-linked kinase, or protein kinase C $(P K C)^{346-352}$. MLC kinase activity is inhibited by $\mathrm{Ca}^{2+} / \mathrm{Cal}$ modulin-dependent protein kinase $\mathrm{I}^{353}$. Many of these kinases are closely associated with or even binding to actin filaments, indicating that actin filaments integrate signaling pathways by facilitating interactions which regulate their own contractile function ${ }^{354}$. In vitro, phosphorylation of MLC can also be accomplished by several kinases which are $\mathrm{Ca}^{2+}$-independent, like Rho kinase, integrin linked kinase and ZIP-like kinase ${ }^{355-357}$. Whether these kinases play a role in physiological SMC contraction is not established at present.

Relaxation of SMCs may occur by reduction of the intracellular $\mathrm{Ca}^{2+}$ concentration through either a decrease of the contractile stimulus, or active extrusion of $\mathrm{Ca}^{2+}{ }^{258}$. However, SMC relaxation can also be achieved in the presence of high $\mathrm{Ca}^{2+}$ levels, by reducing the $\mathrm{Ca}^{2+}$-sensitivity of the SMC contractile machinery ${ }^{359}$. This is mediated by cyclic nucleotide-dependent signaling pathways which lead to the activation of protein kinases like PKA or PKG. CAMP/PKA mediate SMC relaxation induced by $\beta$-adrenergic compounds, whereas CGMP/PKG are involved in relaxation that is triggered by NO and natriuretic peptides. In both cascades, the activity of 
MLC phosphatase and MLC kinase appear to be modulated to achieve the reduction of $\mathrm{Ca}^{2+}$-sensitivity. In addition, inhibition of PKC activity seems to play a role.

\section{Smooth muscle contraction regulation by filament-associated proteins}

A lot of data on SMC contraction indicate that mechanisms distinct from those that directly regulate myosin phosphorylation contribute to the contractile response of SMCs. This is also the case for skeletal muscle contraction, which is regulated by troponins. These are actin filament-associated proteins that cooperate with tropomyosin, providing a $\mathrm{Ca}^{2+}$-switch to contraction. Together, these proteins ensure a coordinated contraction by conferring cooperativity upon the many myosin heads along the actin filaments ${ }^{354}$. It might be expected that SMC contraction is regulated in a similar way. SMCs indeed contain several tropomyosin isoforms ${ }^{42,360}$. However, SMCs do not express troponin. Hence, SMC-specific contractile filament-associated proteins that may substitute the physiological role of troponin have been suggested to contribute to the specific characteristics of $S M C$ contraction. These proteins include h-caldesmon, SM-calponin, SM22 $\alpha$, telokin, and paxillin.

Of these proteins, only h-caldesmon and SM-calponin have been shown to interact with tropomyosin ${ }^{107.361}$. H-caldesmon also interacts with calmodulin, $\alpha$-SMA, and myosin ${ }^{107}$. As such, it has the ability to affect SMC contraction in multiple ways ${ }^{354}$. The best defined effect of h-caldesmon is its ability to reduce myosin ATPase activity, which inhibits contraction ${ }^{362}$. After phosphorylation of $h$-caldesmon by ERK or PKC, or binding of $\mathrm{Ca}^{2+} / \mathrm{calmodulin}$, this inhibition is reversed. Knocking down hcaldesmon in organ cultures of SM tissue or in single SMCs resulted in an increase of basal contractile tone, indicating that $h$-caldesmon indeed suppresses contraction ${ }^{363,364}$. H-caldesmon also functions as a nucleation point for myosin filament assembly ${ }^{365}$.

Like h-caldesmon, SM-calponin binds regulators of contraction, like calmodulin, as well as the contractile proteins themselves. SM-calponin has been shown to inhibit myosin ATPase activity ${ }^{362,366}$. However, the inhibitory action of $h$-caldesmon but not that of $S M$-calponin is enhanced by $S M$ - $\alpha$-tropomyosin, making it likely that these proteins act in different ways ${ }^{75,367}$. Indeed, the effects of SM-calponin seem to be partly due to conformational changes of actin ${ }^{368}$. A more important effect of SMcalponin in regulating SMC contraction may be associated with its role in signal transduction processes. SM-calponin is phosphorylated by Rho kinase and translocates to the cell membrane during contraction, where it links ERK and PKC to the plasma membrane and activates PKC $\mathrm{PC9}^{36-374}$. The role of SM-calponin in smooth muscle contraction at the tissue level has been investigated using SMcalponin knockout mice. When comparing the contractile properties of SMcalponin knockout mice with those of wild-type mice, several differences were detected $^{72}$. First of all, the shortening velocity of permeabilized smooth muscle strips of knockout mice was significantly increased because of enhanced crossbridge cycling. In addition, $\alpha$-SMA levels were reduced by $25-50 \%$ in SM- 
calponin knockouts, possibly leading to fewer or shorter sarcomeres. Also, a different $\mathrm{h}$-caldesmon isoform was expressed in the knockout mice and SM- $\alpha$ tropomyosin levels were reduced by $50 \%$.

Since SM22 $\alpha$ is homologous to SM-calponin and also able to bind to $\alpha$-SMA filaments, a role of this protein in SMC contraction is anticipated. It has been suggested that SM22 $\alpha$ organizes the distribution of actin filaments in SMCs ${ }^{8}$. Indeed, SM22 $\alpha$ knockout mice have an altered $\alpha$-SMA organization ${ }^{68}$. Surprisingly, this does not lead to altered contractile characteristics. This may be explained by functional redundancy provided by the structurally similar $S M 22 \beta$ protein or the paralog SM22 $\alpha$-homolog ${ }^{375,376}$. Indirect evidence that SM22 $\alpha$ does affect SMC contraction is that PKC, which is involved in regulation of other contractile proteins, can phosphorylate $S M 22 \alpha$, thereby preventing interaction of SM22 $\alpha$ with $\alpha-S M A^{377}$. Clearly, the role of SM22 $\alpha$ in SMC contractile function needs much more study.

The involvement of telokin in the regulation of SMC contraction, in contrast, is evident. Telokin is a small cytoplasmic protein which is identical to the C-terminal part of MLC kinase. Telokin promotes myosin polymerization, inducing filament extension ${ }^{84,365}$. Yet it appears that its effects on myosin phosphorylation, i.e. increasing relaxation, are more significant ${ }^{89}$. The mechanism by which telokin acts is unclear, but several theories have been put forward. These include a direct competition for myosin between MLC kinase and telokin ${ }^{378}$, and increased MLC dephosphorylation by telokin, possibly enhanced by its own phosphorylation ${ }^{379}$. This latter mechanism is presumably related to activation of MLC phosphatase ${ }^{89}$. There are also reports claiming that telokin directly inhibits MLC kinase ${ }^{380,381}$. Telokin phosphorylation by PKG and MAPK upon activation of cGMP signaling pathways leads to its translocation towards the plasma membrane ${ }^{84}$. However, phosphorylation does not seem to be related to the relaxing effects of telokin ${ }^{382,383}$. Thus, although telokin clearly affects SMC contraction dynamics to promote SMC relaxation, its exact mode of action is still unclear.
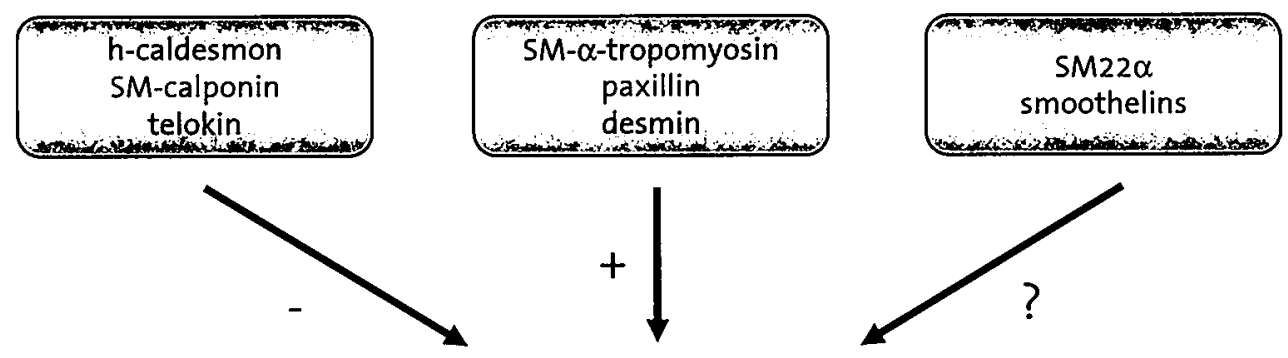

\section{Contraction}

Figure 1.13 SMC contraction regulation. Filament-associated proteins and their effects on SMC contraction. 
The dense plaque-associated protein paxillin was recently shown to regulate SMC contraction ${ }^{384}$. Paxillin associates with actin filaments and is phosphorylated after activation of contraction. Paxillin depletion has been shown to lead to diminished tension in SM strips, without altering $\mathrm{Ca}^{2+}$-levels, MLC phosphorylation, or myosin ATPase activity. However, $\mathrm{G}$-actin levels were markedly reduced in resting paxillin depleted muscle. Therefore, it was suggested that paxillin might regulate polymerization and depolymerization of actin. As discussed below, remodeling of actin filaments may serve to optimize force development at different lengths of the cell.

The summarized reports point to the importance of SMC contraction regulation by filament-associated proteins (figure 1.13). Since not nearly all of the specific SMC contractile characteristics can be explained by these results at present, it is likely that additional roles for the proteins discussed continue to be discovered. Moreover, other contractile filament-associated proteins like smoothelins, of which the function is still unknown, may be responsible for certain SMC contractile characteristics.

\section{Latch: smooth muscle contraction maintenance}

One of the characteristic aspects of SMC contraction is that it can be maintained with little use of ATP. This phenomenon has been called 'latch', and is characterized by reduced cross-bridge cycling ${ }^{385}$. How the latch phenomenon can be explained at the molecular level is unknown. Yet the fact that during sustained contractions $M L C_{20}$ phosphorylation and $\mathrm{Ca}^{2+}$-concentration drop to low levels indicates that mechanisms distinct from myosin phosphorylation are responsible for maintaining contraction ${ }^{5}$.

Mechanisms for sustained contraction may involve cooperative attachment of dephosphorylated but ADP-bound cross-bridges, or dephosphorylation of attached cross-bridges, which detach much slower than phosphorylated cross-bridges and consequently prolong the duration of contraction ${ }^{386}$. SM-calponin and h-caldesmon have been suggested to be involved in regulating this cooperative attachment of dephosphorylated cross-bridges ${ }^{351}$. In addition, h-caldesmon has been suggested to be able to cross-link actin to myosin filaments, thereby forming a force-bearing non-cycling cross-bridge which could also explain the latch state ${ }^{387}$. Apart from these mechanisms, rearrangement or phosphorylation of cytoskeletal proteins like desmin may also contribute to sustained SMC contraction, possibly by tethering myosin and actin filaments after the generation of force. Finally, the existence of different populations of myosin cross-bridges has been suggested to explain the latch phenomenon. Results from SM-MHC-B knockout mice indicate that the relative contribution to sustained contraction differs between myosin isoforms. These mice do not show the rapid phasic way of contraction, but are able to contract and maintain force. Thus, cross-bridges contributed by SM-MHC-B isoforms may be responsible for fast cycling, whereas other myosins, e.g. SMemb, represent the other, more slowly cycling population which could be responsible for 
the latch state ${ }^{65,66}$.

\section{Rearrangement of contractile filaments}

In addition to modulation of SMCs between synthetic and contractile phenotypes, there exists another level of plasticity in contractile SMCs. This so-called mechanical plasticity is responsible for the property that the contractile force that a SMC can generate is almost independent of its length ${ }^{5}$. This is required for the function of a SMC in vivo, as the mechanical forces working on the SMC are constantly changing through breathing, feeding, cardiac pumping, or through contraction by other SMCs, and SMC length has to change accordingly ${ }^{388}$. Changes in smooth muscle length temporarily diminish the force that can be produced. This is not correlated with the phosphorylation state of $\mathrm{MLC}_{20}{ }^{389}$, and thus probably not caused by deactivation of the contractile machinery. It has been proposed that the phenomenon is caused by a transient disorganization of the contractile apparatus ${ }^{390}$. Others have suggested that instead of disorganization, this is actually a process of physiological reorganization. In their view, after stretching a SMC, the contractile filaments are rearranged in such a way that more contractile units are placed in series ${ }^{388}$. After shortening of the cell, the reverse process takes place, enabling the cell to always generate maximal force, albeit at the cost of shortening velocity. This hypothesis is supported by a study showing that SMCs can only exert maximal force again after reorganization of the contractile filaments has been completed $^{391}$. Myosin filament remodeling seems to take place after each contraction-relaxation cycle ${ }^{391}$. Remodeling of actin filaments during contraction of A7r5 SMCs has also been demonstrated ${ }^{392}$. Observations on the contractile characteristics of HITB5 SMCs support the filament rearrangement theory. During modulation towards a contractile phenotype, these cells gain the capacity to contract even though there is only a modest increase in contractile protein expression $^{157}$. It is likely that reorganization of existing myofilaments plays a significant role in ensuring maximal contractile potential at diverse SMC lengths. This phenomenon explains why smooth muscle is actually 'smooth', that is, why there are no striations: the contractile network is constantly changing.

RhoA has been suggested to regulate mechanical plasticity of SMCs. It is induced by repeated stretch of SMCs, and subsequently regulates both actin and myosin filament assembly ${ }^{356.393-397}$. As discussed before, Rho kinase can phosphorylate MLC phosphatase, thereby inhibiting its myosin-binding subunit. This causes an increase in $\mathrm{MLC}_{20}$ phosphorylation, which has a positive effect on the incorporation of SMMHC monomers in myosin filaments ${ }^{398.399}$. In addition, RhoA overexpression enhances stress fiber formation in cultured $S \mathrm{MCs}^{286}$. Overexpression of constitutively active Rho kinase alone is not as effective in generating stress fibers as RhoA itself, indicating that other targets of RhoA are involved ${ }^{400}$. One such target is mDia1, which seems to direct the actin-monomer binding protein profilin to sites where RhoA is active, thereby promoting actin polymerization ${ }^{401}$. Collectively, these 
data suggest that RhoA is an important regulator of contractile filament remodeling in SMCs. Simultaneously, it enhances transcription of the proteins that form these filaments, in addition to proteins that regulate SMC contraction (see figure 1.8). Thus, levels and organization of contractile proteins may be tuned by RhoA.

\section{Smooth muscle cell contraction modes}

SMCs have been categorized as tonic or phasic, according to the nature of their contraction. Phasic SMCs are characterized by high rates of force activation and relaxation, high maximum speeds of shortening, and poor force maintenance. Tonic SMCs, in contrast, display a slow rate of force activation and relaxation, lower maximum speeds of shortening, but good force maintenance ${ }^{402}$. These two types of contraction can be uncoupled, as evident from their differential inhibition by a Rho kinase inhibitor ${ }^{351}$. Generally, visceral SMCs and vascular SMCs of the smaller arteries and larger veins show only phasic contractions, whereas bronchial SMCs and vascular SMCs of the larger arteries contract in a tonic way. These different modes of contraction are associated with a different subcellular organization of the organelles involved in contraction. The peripheral SR of phasic SMCs is for example more developed than the perinuclear SR, whereas in tonic SMCs the opposite is seen. This may relate to the fact that close association of the SR with extracellular stimuli facilitates generation of fast phasic contractions ${ }^{5}$.

The different mechanical properties of tonic and phasic SMCs may be brought about by either differences in signal transduction mechanisms or differences in the effector contractile proteins. There are many reports on putative determinants of contraction profiles of SMCs. For instance, phasic SMCs have been shown to be less sensitive to $\mathrm{Ca}^{2+}$-levels and MLC phosphatase inhibitors than tonic SMCs, and to contain higher amounts of MLC kinase and phosphatase ${ }^{403}$. This is compatible with a high turnover of phosphorylated $\mathrm{MLC}_{20}$, which is characteristic of the phasic mode of contraction. Calmodulin and calmodulin-binding protein levels have also been suggested to affect the contractile behavior of SMCs. Indeed, activity of MLC kinase and the concentration of both calmodulin and h-caldesmon is higher in phasic SMCs ${ }^{404}$.

Alternatively, different isoforms or splice variants of contractile proteins may be associated with a phasic or tonic contraction profile. Especially the subunits of $S M$ myosin have been implicated in this respect, since SM-MHC-B isoforms appear to be important for phasic contraction ${ }^{65,66}$. $\mathrm{MLC}_{17}$ isoforms are also associated with the nature of $S M C$ contraction. The splice variant which gives rise to the acidic isoform $\left(M L C_{17 b}\right)$ is predominant in tonic vascular SMCs, whereas $M L C_{17 a}$, which is the basic isoform, is expressed at higher levels in phasic SMCs ${ }^{402}$. Accordingly, introduction of $M L C_{17 b}$ in rabbit bladder phasic SM strips leads to a lower rate of force development and shortening velocity than introduction of $M C_{17 a}{ }^{405}$. Differential affinity for MgADP, resulting in different ATPase activity, is responsible for this effect. Overexpression of the 'phasic' or 'tonic' isoform of $\mathrm{MLC}_{17}$ in cultured aorta or 
gizzard SMCs showed the same results ${ }^{406}$.

In conclusion, the different ways of SMC contraction are in a complex and incompletely understood way regulated by both $\mathrm{Ca}^{2+}$-dependent and $\mathrm{Ca}^{2+}$ independent mechanisms. Much work will need to be done before the characteristics of SMC contraction can be explained at the molecular level. Knocking down components of the contractile machinery by short interfering RNA or conditional knockout approaches may help to reveal their importance for the characteristics of SMC contraction at the cellular respectively the tissue level. In addition, yeast two hybrid systems may be employed to identify new proteins involved in SMC contraction that bind to established contraction regulators.

\section{Synthetic function of the smooth muscle cell: structural support}

Apart from their contractile functions, SMCs generate and organize many different ECM compounds. These compounds provide a supporting, structural framework for smooth muscle tissues that is essential for their integrity, making them comply with stretch.

\section{Extracellular matrix components synthesized by smooth muscle cells}

The two most abundant ECM proteins in smooth muscle tissue are collagens and elastin, but proteoglycans and structural glycoproteins also play a major part in determining the functional properties of this structural lattice ${ }^{407}$. Nineteen different collagens have been described to date, of which type I and type III are the major isoforms in the ECM of smooth muscle tissues ${ }^{407}$. Collagens are synthesized as monomers and aggregate as triple helices into collagen fibrils. The organization of these fibrils is variable, depending on the type of collagen, presence of other ECM molecules, stage of differentiation of the SMC, and local mechanical factors ${ }^{408}$. After formation of the fibrils, they are crosslinked by lysyl oxydase, which makes them more insoluble and more resistant to tensile force.

Elastin, which is particularly abundant in large arteries, is essential for maintaining the structural integrity of smooth muscle tissues by providing the elasticity to cope with constantly changing pressure. Elastin represents $90 \%$ of the elastic fibers of smooth muscle tissue, while the remaining $10 \%$ is constituted by fibrillins and microfibrillar-associated glycoproteins ${ }^{407}$. Like collagen fibrils, elastin is formed by crosslinking of monomers, i.e. tropoelastin molecules. Elastin knockout mice die because their arteries are obstructed by excessive SMC proliferation, a reaction to the compromised structural integrity ${ }^{409}$.

Fibrillins are glycoproteins that are closely associated with elastin in elastic fibers. Other glycoproteins include fibronectin, vitronectin, laminin, tenascin, and thrombospondin. These proteins mediate interactions between the SMCs and other ECM compounds. Proteoglycans are ECM compounds that are posttranslationally 
modified by addition of glycosaminoglycan chains ${ }^{407}$. They take part in the assembly of the ECM and play various roles in the control of for example filtration and hydration of tissues, and migration of SMCs. Many different proteoglycans have been found in the ECM of SMCs, including for example versican, syndecan, and hyaluronic acid.

\section{Extracellular matrix balance}

Turnover of ECM proteins in adults is generally slow. However, for controlled remodeling of the ECM, SMCs synthesize matrix degrading proteases and their inhibitors. Many different types with discrete substrate specificities exist. Collagens are mainly degraded by MMPs, whereas elastin is broken down by general proteinases of the serine, metallo-, aspartic, and cysteine classes ${ }^{407}$. MMPs include collagenases (MMP-1, MMP-8), gelatinases (MMP-2, MMP-9), elastases (MMP-7, MMP-12), stromelysins, and membrane-type metalloproteinases. MMP activity is the outcome of regulated expression, secretion, and activation of MMP proenzymes on the one hand, and inhibition by TIMPs on the other hand. In physiological situations, MMPs are constitutively expressed, but the balance between activating and inhibiting enzymes is tightly controlled so that they have a very low activity. In pathological processes, activating enzymes secreted by SMCs or inflammatory cells enhance MMP activity. SMCs can modulate this response by increased synthesis of TIMPs. For example, elastin degradation caused by increased MMP-2, MMP-9, and MMP-12 synthesis is observed in aneurysms, although TIMP expression is also higher in aneurysmal tissues ${ }^{407}$. In hypertensive patients, collagen and elastin content of arteries is increased, and their degradation is reduced by increased TIMP expression ${ }^{410,411}$. It is clear that reorganization of the ECM by MMPs and TIMPs is a continuous, highly regulated process, which is of great importance for the integrity of SM tissues. A recent review by Visse $\&$ Nagase provides an extensive overview of the role of these proteins in ECM remodeling ${ }^{412}$.

\section{Smooth muscle cell adhesion molecules}

Apart from their structural functions, the components of the ECM also relay signals that control the behavior of the SMC with respect to proliferation, migration, differentiation, and surviva ${ }^{408}$. Signals from the ECM are transmitted into the SMC by adhesion molecules that are connected to the SMC cytoskeleton ${ }^{413}$. These linker proteins fortify the adhesion of SMCs to the ECM, and simultaneously allow the ECM to affect gene expression patterns of the SMC. Adhesion receptors of SMCS include various integrins, syndecans, vascular- and intercellular cell adhesion molecules ( $V$ - and ICAMs), cadherins, and the dystrophin-glycoprotein complex. Integrins are the transmembrane receptors for the ECM, linking the ECM with actin and the cytoskeleton. They are composed of $\alpha$ - and $\beta$-subunits with specific ligandbinding properties. Integrins occur in various combinations in the cell membrane of the SMC. The $\alpha 1 \beta 1, \alpha 3 \beta 1, \alpha 5 \beta 1, \alpha v \beta 1$, and $\alpha v \beta 3$ variants are most abundant. These complexes interact with different ECM components including collagens, laminins, 
and fibronectin, and mediate the reaction of SMC phenotype on stretch and flow ${ }^{414}$. The dystrophin-glycoprotein complex provides mechanical stability to the SMC membrane during contraction ${ }^{225}$. It links the $S M C$ basement membrane to the actin filaments and consists of $\alpha$ - and $\beta$-dystroglycans, $\beta$-, $\delta$-, and $\varepsilon$-sarcoglycans, sarcospan, and dystrophin. Syndecans are transmembrane receptors with glycosaminoglycan chains. They act as co-receptors by providing additional sites for ECM ligands, thereby increasing cell adhesion strength ${ }^{415}$. In addition, they bind to several growth factors among which FGF and VEGF, possibly mediating proliferation of SMCs. ICAM-1 and VCAM-1 are thought to mediate adhesion of inflammatory cells in atherosclerosis ${ }^{225}$. SMCs also express different types of cadherins, including $\mathrm{N}-, \mathrm{R}-$, and T-cadherin. Readers are referred to the recent review by Moiseeva ${ }^{225}$ for more information on the function and expression of adhesion molecules expressed in SMCs.

\section{Synthetic smooth muscle cell function in disease and remodeling}

The functions of the SMC in physiological remodeling of smooth muscle tissues and their repair after injury require an initial response of proliferation, migration, and matrix synthesis, with subsequent gain of contractile performance. Thus, under these conditions, SMCs transiently adopt a more synthetic phenotype. This response has been mostly studied by models of neointimal thickening in restenosis and atherosclerosis. In these disorders, an accumulation of SMCs with a synthetic phenotype is observed. Restenosis occurs for example after percutaneous transluminal coronary angioplasty (PTCA) or stenting of an artery. This procedure damages the endothelial cells that line the vessel wall, thereby exposing the SMCs to factors that stimulate phenotypic modulation. After modulation towards the synthetic phenotype, proliferation of medial SMCs is observed which is followed by migration out of the media to the intima of the vessel. This accumulation of SMCs leads to the formation of a neointima. These neointimal SMCs frequently continue to proliferate, extending the lesion and occluding the lumen. After reendothelialization of the vessel, phenotypic modulation of the neointimal SMCs towards the contractile phenotype may occur. Atherosclerosis is partly characterized by these same processes, but the accumulation of SMCs in atherosclerosis is much slower and the involvement of other cell types makes this disease much more complex as compared to restenosis.

\section{Characteristics of neointimal smooth muscle cells}

Neointimal SMCs differ from SMCs in the tunica media of the same vessel with respect to morphology and expression of contractile proteins, integrins, ECM molecules, and growth factors ${ }^{13}$. However, the factors that regulate medial SMC phenotype also act on intimal SMCs. For example, TGF- $\beta$ has been shown to induce ECM deposition by SMCS in the neointima, and retinoids have been shown to 
inhibit neointimal formation after arterial injury $203,416,417$.

Cloned SMCs from early neointimal lesions mostly have an epithelioid or rhomboid morphology and low levels of contractile protein ${ }^{12}$. However, after few weeks, the neointimal SMCs again resemble spindle-shaped cells, indicating modulation towards a more contractile phenotype ${ }^{18}$. Indeed, around 60 days after injury, $\alpha$ SMA, SM-MHC, and desmin expression and contraction potential of intimal SMCS are similar to that of the underlying medial SMCs ${ }^{418}$. Besides, the SMC content of the intimal thickening gradually decreases via apoptosis ${ }^{15}$.

Both spindle-shaped and epithelioid SMCs have been found in human atherosclerotic lesions ${ }^{419}$. In addition, markedly different gene expression profiles have been found in intimal SMCs of the same atherosclerotic plaques, implying different SMC phenotypes ${ }^{76}$. Interestingly, SMCs in the so-called fibrous cap of the plaques sometimes expressed high amounts of some contractile proteins compared to apparently contractile SMCs in the media adjacent to the lesions. However, SM-calponin was never found in intimal SMCs, whereas SM22 $\alpha$ was only present in intimal SMCs in the fibrous cap of advanced lesions. ECM proteins were expressed to more or less the same extent. SMCs in advanced atherosclerotic plaques have been shown to differ from SMCs of early lesions. The former SMCs express high levels of fibroblast surface protein (FSP), in contrast to SMCs of early lesions ${ }^{420}$. The FSP-positive SMCs did not express desmin, contained variable amounts of $\alpha-S M A, h$-caldesmon, and SM-MHC, and were more proliferative than the FSP-negative cells.

Many proteins that are associated with the synthetic SMC phenotype, including different collagen types, hyaluronan, and MMPs, have higher expression levels in intimal SMCs ${ }^{22,96,216,421}$. Collectively, expression of these proteins allows the SMCs to migrate and proliferate to a greater extent ${ }^{422}$. Contrary to expectations, downregulation of contractile gene expression is not necessarily observed in proliferating neointimal SMCs ${ }^{136}$. Rather, simultaneous upregulation of genes associated with both the contractile and the synthetic phenotype is frequently demonstrated. Because of this, it has been suggested that SMC gene expression during vascular repair resembles the phenotype seen during the neonatal period ${ }^{136}$. In support of this, neonatal SMCs and neointimal SMCS have a similar morphology $y^{12,136,423}$.

\section{Neointimal smooth muscle cell origin}

The origin of neointimal SMCs is still a matter of debate. Currently, three theories exist to explain the origin and characteristics of SMCs of the neointima. First, they could be derived from contractile SMCs in the media that have migrated after phenotypic modulation ${ }^{12}$. Alternatively, neointimal SMCs could be the result of expansion of SMCs that already had a synthetic phenotype ${ }^{424}$. Indeed, a proportion of SMC clones obtained from the normal adult media is phenotypically similar to SMCs cloned from the neointima ${ }^{12}$. There is in vitro data in support of the view that distinct subsets of SMCs respond to different stress factors like PTCA or shear 
stress ${ }^{155}$. It has even been suggested that a particular SMC subpopulation with an inherent higher proliferative potential may contribute to intimal thickening by inducing modulation of other SMC populations that are by nature more resistant to modulation through secretion of mitogens. In a physiological situation, these proliferation-sensitive SMCs may be kept quiescent by secretion of inhibitory factors by the proliferation-resistant SMC subsets. After injury, the balance changes, allowing for the appropriate response by the proliferation-sensitive SMCs. The third possible origin of neointimal SMCs is transdifferentiation of endothelial cells or circulating immune cells ${ }^{143}$. These theories do not exclude each other. It may even be expected that all three mechanisms are necessary for an adequate response of SMCs to injury. Theoretically, medial SMCs that already had a synthetic phenotype can respond rapidly, simultaneously inducing modulation of contractile SMCs and providing an environment in which blood-borne SMC precursors can further differentiate towards functional SMCs.

\section{Regulation of smooth muscle cell phenotype in disease}

The contributions of specific transcription factors or cis-elements to the regulation of the response of SMCs to injury are only beginning to be elucidated. It is likely that many of the factors and elements that have been shown to control SMC differentiation and phenotypic modulation in physiological situations also control the SMC response in disease.

One of these factors, GATA-6, is downregulated after PTCA of the arterial wall ${ }^{425}$. After adenovirus-mediated GATA- 6 expression in a rat PTCA model, there were $50 \%$ less neointimal SMCs. Neointimal SMCs that did develop had a more contractile phenotype than controls. Interestingly, this effect of GATA- 6 was not observed after infection of cultured SMCs, underscoring the differences in differentiation pathways in vivo and in vitro. Other important factors regulating SMC phenotype in disease may be myocardin, which is downregulated after arterial injury, and MEF2 isoforms, which are increased ${ }^{39,303}$. Mutation of a Sp1 element in the SM22 $\alpha$ promoter has been shown to inhibit the downregulation of this gene that normally occurs after vascular injury ${ }^{59}$. This element is also present in the SM-MHC promoter ${ }^{426}$ and the ACLP promoter ${ }^{96}$. Other more general transcription factors that influence SMC proliferation in disease are AP-1 and NFKB. Inhibition of AP-1 by oligo decoys attenuated neointimal formation after PTCA of the carotid artery of the rat $^{324}$, and NFKB pathways are activated in SMCs in the vessel wall after injury ${ }^{427}$.

Proteins that have been shown to control neointimal SMC numbers are Rb, p21, and $p 27^{307,428}$. TGF- $\beta$-induced upregulation of $p 21$ and $p 27$ has been reported to inhibit SMC proliferation and migration after angioplasty, leading to reduced neointimal formation ${ }^{429-431}$. The antiproliferative and antimigratory effects of p27 are mediated by cyclin-dependent kinases and accumulation of hypophosphorylated $\mathrm{pRb}$, suggesting that these proteins may act as coordinators of these two distinct processes. In recent years, reduction of restenosis after 
stenting has been achieved by targeting the proliferation of SMCs at the transition from the G1 phase to the S phase of the cell cycle ${ }^{432}$.

It is clear that the main difference between healthy and diseased smooth muscle tissues is the proportion of synthetic SMCs. Whereas in physiological situations the balance between contractile and synthetic SMCs is tightly controlled in favor of the contractile phenotype, this balance is deregulated by external stimuli in diseased tissues. When synthetic SMCs predominate, rigid extracellular material accumulates and contractile protein levels diminish, leading to compromised contractile performance. Moreover, since synthetic SMCs proliferate more actively, the lumen of diseased smooth muscles frequently becomes occluded. Some of the factors that affect SMC behavior in disease are now known. Increased understanding of the mechanisms by which these factors act and the identification of additional molecules that play a role will allow a rational therapeutic approach of these disorders. This treatment should be aimed at restoration of the balance between contractile and synthetic SMCs that is normally observed in smooth muscle tissues. 


\section{Smoothelin, a new entry to the study of smooth muscle cell function and differentiation}

\section{Characteristics of smoothelins}

\section{Smoothelin expression}

Smoothelin was first described by van der Loop et al. (1996) as a cytoskeletal protein with SMC-restricted expression ${ }^{23}$. Expression of the $59 \mathrm{kDa}$ protein appeared to be associated with the contractile phenotype of the SMC, since a rapid loss of smoothelin mRNA in smooth muscle tissue explants was observed, and smoothelin mRNA could not be detected in cultured SMCs. Smoothelin protein turnover seemed to be quite slow, as it could be found in primary SMCs up to 5 days after explantation, long after its mRNA expression was shut down. During prolonged culturing, smoothelin levels and the number of smoothelin-positive cells gradually declined. The human smoothelin gene, which has been designated SMTN, is a single copy gene localized to chromosome 22912.3 , as shown by fluorescence in situ hybridization and radiation hybrid mapping ${ }^{433}$.

\section{Subcellular localization of smoothelin}

Smoothelin has been shown to be organized into a filamentous structure in embryonic chicken gizzard SMCs, and to colocalize to some extent to $\alpha$-SMA in the stress fibers of these cells ${ }^{23}$. It did not colocalize with desmin, vimentin, keratin, or tubulin. Another research group indicated that smoothelin did colocalize with desmin and filamin, although only partially overlapping staining patterns were shown ${ }^{434}$. Transfection of smoothelin CDNA in human primary SMCs and rat heartderived myoblasts and subsequent immunofluorescence analysis using the antismoothelin antibody R4A confirmed the $\alpha$-SMA-associated filamentous staining pattern ${ }^{23}$. Transfection in COS7 cells, which do not contain stress fibers, showed that smoothelin can form a filamentous network structure by itself. Smoothelin can be extracted from SMCs together with actin-binding proteins like myosin, vinculin, filamin, and $\beta$-actin, but not with desmin ${ }^{434}$.

\section{Smoothelin protein variants}

Smoothelin is a basic protein with a pl around 8.2 and approximately five isoelectric variants as detected in two-dimensional gel electrophoresis ${ }^{434}$. Smoothelin has been shown to be glycosylated, which contributes approximately $10 \mathrm{kDa}$ to its MW. It is conserved across species that are evolutionary far apart, indicating an important function ${ }^{23}$. However, only one functional domain in the protein could be identified by homology searches. This concerned a C-terminal motif resembling the actin-binding sequence of proteins belonging to the spectrin superfamily. Some regions capable of adopting an $\alpha$-helical structure, and a proline-rich region were also detected in the protein. 
After the initial identification and characterization of smoothelin, it became clear that a second smoothelin isoform exists. This $110 \mathrm{kDa}$ smoothelin, first described as a $94 / 95 \mathrm{kDa}$ protein and now designated smoothelin-B, has been shown to be predominantly expressed in SMCs of vascular origin ${ }^{80}$. It is not formed by dimerization of the lower $M W$ smoothelin, which is now referred to as smoothelin$A^{434}$.

\section{Distribution of smoothelin in the vasculature}

The data from the study by van der Loop et al. suggested that smoothelin expression was restricted to contractile $\mathrm{SMCs}^{23}$. To further support this notion, smoothelin expression was investigated in human vascular tissues with different contractile characteristics ${ }^{79}$.

Smoothelin was detected in developing arteries of the placenta from 10-11 weeks of gestation onwards, but not at week 7-8. Fetal veins in the placenta were still negative after 10-11 weeks. Most vessels of the term placenta and all vessels of term umbilical cord contained smoothelin. In adult elastic arteries like the aorta, smoothelin was only detected in 5-10\% of medial SMCs and not in intimal or adventitial cells. Smoothelin expression in muscular arteries was considerably higher. The medial layer of large $(2-10 \mathrm{~mm})$ muscular arteries contained $30-50 \%$ smoothelin-positive SMCs, whereas this number increased to $>95 \%$ in small $(0.3-2$ $\mathrm{mm}$ ) muscular arteries. In addition, SMCs of the vasa vasorum in the adventitia of large muscular arteries were shown to express smoothelin, and large veins contained 1-3 layers of smoothelin-expressing SMCs. In contrast, smoothelin could not be detected in capillaries, venules, and small veins. In SMCs of arteriovenous anastomoses, only low smoothelin expression was detected by immunofluorescence ${ }^{435}$. These epithelioid cells represent a specialized synthetic SMC-type that contains many organelles and loosely arranged myofilaments. All in all, the observed distribution of smoothelin-positive SMCs indicates that there is a correlation between smoothelin expression and smooth muscle contractility.

Since the phenotype of many SMCs in vascular disease changes from predominantly contractile to mainly synthetic, smoothelin expression in diseased vascular SMCs was also investigated ${ }^{79}$. Aortic aneurysmal SMCs did not contain smoothelin-positive SMCs. Early atherosclerotic lesions also did not contain SMCs that expressed smoothelin. However, in 10-20\% of advanced atherosclerotic lesions smoothelin expression was observed, especially in SMCs at the luminal side of the lesion. 


\section{Aim of the thesis}

The identification and characterization of SMC marker proteins has greatly facilitated progress in the field of SMC differentiation and phenotypic modulation. However, major advances in our understanding of the contractile SMC phenotype and its regulation have been hampered by the lack of markers of the advanced contractile SMC phenotype. Likewise, although the unique features of SMC contraction are increasingly understood, not all of its properties can be explained on the basis of known contractile proteins and their regulators.

The discovery of the smoothelins opened up new avenues in these respects. Smoothelins were shown to be highly restricted to SMCs and thus represented a new addition to the SMC marker protein set. Moreover, they appeared to be specific to the contractile SMC phenotype and consequently could be useful for the study of this phenotype as well as SMC phenotypic modulation. In addition, the exclusive expression of smoothelins in contractile SMCs and their colocalization with the SMC contractile apparatus suggested that they might play a role in SMC contraction. Since the initial characterization of smoothelins was not sufficient to establish their possible role in SMC function or their value as contractile SMC marker proteins, however, the overall aim of the studies described in this thesis was:

\section{To increase our understanding of the expression, regulation, and function of smoothelins.}

In order to determine the value of smoothelins as markers to monitor SMC differentiation and phenotypic modulation, we first focused on their expression pattern in modulating cultured SMCs and in developing SMCs. The behavior of smoothelin-B relative to several more fully explored contractile SMC markers was determined in a novel pig coronary artery model of SMC differentiation (chapter 2). In addition, the expression pattern of the smoothelins during embryogenesis was investigated in the chicken (chapter 3 ).

To explain the tissue- and phenotype-specific expression of the smoothelins, the regulation of their expression pattern was addressed next. Several mechanisms that could be responsible for the generation of distinct smoothelin isoforms were analyzed (chapter 4). Promoter regions in the smoothelin gene were identified and the importance of specific transcriptional regulators and cis-elements in these regions for smoothelin isoform expression was assessed (chapter $4 \& 5$ ).

Functional characterization of smoothelins by in vitro techniques was impeded by the rapidly declining smoothelin levels in cultured SMCs and the insolubility of the proteins. Therefore, functional studies on smoothelins required an in vivo approach. To allow in vivo studies on the function of smoothelins by gene targeting, the mouse smoothelin gene was sequenced (chapter 6). Subsequently, smoothelin-deficient mice were generated and their physiology and pathology was 
investigated to reveal a critical role of smoothelins in SMC contractile function (chapter 7). The results obtained in these studies and their implications for future studies on the role of smoothelins in SMC contraction and the definition of the SMC phenotype are discussed in chapter 8. 


\section{2}

\section{Cultured porcine coronary artery smooth muscle cells: a new model with advanced differentiation}

Thomas Christen*, Marie-Luce Bochaton-Piallat*, Pascal Neuville*, Sander Rensen**, Mireille Redard*, Guillaume van Eys**, Giulio Gabbiani*

* Department of Pathology, University of Geneva-CMU, Geneva, Switzerland *- Department of Genetics and Cell Biology, Cardiovascular Research Institute Maastricht, Universiteit Maastricht, Maastricht, The Netherlands 


\section{Abstract}

Arterial intimal thickening after endothelial injury induced in rodents has proven to be a relatively unreliable model of restenosis for testing clinically useful compounds. The same has been found for cultured rat or rabbit vascular smooth muscle cells (SMCs). To test alternative possibilities, we have studied several differentiation features of porcine coronary artery SMCs, cultured up to the 5th passage after enzymatic digestion of the media. The effects of heparin, transforming growth factor (TGF)- $\beta_{1}$ or $-\beta_{2}$, and all-trans retinoic acid (tRA) on proliferation, migration, and differentiation of these cells were also examined. Porcine arterial SMCs in culture not only express high levels of $\alpha$-smooth muscle actin $(\alpha-S M A)$, but contrary to rodent SMCs, also maintain an appreciable expression of smooth muscle myosin heavy chain isoform (SM-MHC) 1 and 2 , desmin, and smoothelin, a recently described late differentiation marker of vascular SMCs. We demonstrate for the first time that smoothelin is colocalized with $\alpha$-SMA in these cells. Finally, we show that in the porcine model, heparin is more potent than TGF- $\beta_{1}$ or $-\beta_{2}$ and TRA in terms of inhibition of proliferation and migration and of increasing the expression of differentiation markers. This model should be a useful complement to in vivo studies of SMC differentiation and of pathological situations such as restenosis and atheromatosis.

\section{Introduction}

The arterial intimal thickenings (IT) induced in the rat or rabbit after endothelial lesion are presently the most-studied models for atheroma formation and/or restenosis, and have been useful for defining several biological features of SMCs. However, these models have many important limitations ${ }^{436}$, which could explain the clinical failure of substances that proved to be efficient inhibitors of IT formation in these animal models.

The biological features of SMCs in culture have also been systematically studied using cells derived from rat or rabbit arteries ${ }^{38,437-441}$. However, limitations similar to those observed in in vivo experiments were encountered. Among the models of large animals, the pig coronary artery IT has been used more and more ${ }^{442,443}$. Pigs may develop spontaneously coronary atheromatosis with age, and the induction of typical plaques is easily achieved by a cholesterol-rich diet ${ }^{444,445}$. Furthermore, angioplasty and other interventions can be performed in porcine coronary arteries

with the same instruments as in humans. Although pig aortic and coronary SMCs have already been studied in vitro, no systematic description of their differentiation features during culture has been published.

This report describes the characterization of differentiation features and several biological properties of cultured porcine left anterior descending (LAD) coronary artery SMCs. We show that, contrary to rat or rabbit arterial SMC5 ${ }^{38,437-441}$, porcine 
SMCs maintain a high level of differentiation marker expression in culture, including the hitherto unreported expression of smoothelin, a recently described marker of late SMC differentiation. We describe the replicative and motile properties of these cells as well as the influence of heparin, TGF $-\beta_{1}$ or $-\beta_{2}$, and tRA on these activities. Our findings suggest that porcine coronary artery SMCs retain a much higher level of differentiation and behave differently in vitro from the majority of SMCs previously studied. Thus, they may represent a better model for the study of agents influencing SMC behavior in vitro and in vivo.

\section{Materials and methods}

\section{Cell culture}

Coronary arteries from domestic crossbred pigs (Sus scrofa domesticus) were obtained from a nearby slaughterhouse at the approximate age of 8 months. The $40 \mathrm{~mm}$ of the LAD distal to the branching of the left circumflex coronary artery was chosen for dissection and enzymatic digestion. The vessel was opened by a longitudinal cut. The endothelium was gently scraped with a surgical blade, and the media was then stripped from the underlying adventitia with two ophthalmological tweezers and finely minced. The material of two coronary arteries was pooled and digested for 60 minutes at $37^{\circ} \mathrm{C}$ in $6 \mathrm{ml}$ of Dulbecco's modified Eagle medium (DMEM; Gibco BRL, Life Technologies, Paisley, UK) with 2\% Hepes (Life Technologies) containing $40 \mathrm{mg}$ of collagenase (Sigma-blend collagenase, type F $1800 \mathrm{U} / \mathrm{mg}$; Sigma, St. Louis, MO), $4.2 \mathrm{mg}$ of elastase from hog pancreas (9.7 U/mg; Fluka, Buchs, Switzerland), and $12 \mathrm{mg}$ of soybean trypsin inhibitor (1 mg inhibiting $2.1 \mathrm{mg}$ trypsin; Worthington, Freehold, NJ). The enzymatic activity was stopped by fetal calf serum (FCS Seromed, $10 \%$ final concentration; Biochrom KG, Berlin, Germany), and the suspension was filtered through a sterile gauze to discard remnants. The filtrates of two preparations were pooled and plated on a 100-mm culture dish (Falcon, Becton Dickinson, Lincoln Park, NJ) in DMEM without FCS. Two hours later, FCS was added up to a final concentration of $10 \%$. The medium was changed after 24 hours, and the primary culture was confluent in about six days. Medium was always changed every 3 days. The plating efficiency (ratio of attached/plated cells) at 24 hours was always found to be about $70 \%$. These cells were passed by a $1: 2$ split ratio and studied up to the 6th passage.

\section{Cell treatment}

SMCs at the 5th passage were plated at a density of 60 cells $/ \mathrm{mm}^{2}$ in $100-\mathrm{mm}$ culture dishes containing DMEM supplemented with $10 \%$ FCS. In a first series of experiments, medium was changed 24 hours after plating, and SMCs were incubated alone or in combination with one of the following: $200 \mu \mathrm{g} / \mathrm{ml}$ heparin (H-9399; Sigma), $10 \mathrm{ng} / \mathrm{ml} \mathrm{TGF-} \beta_{1}$ (Sigma), $10 \mathrm{ng} / \mathrm{ml}$ TGF- $\beta_{2}$ (gift of Dr. D.A. Cox, 
Novartis, Basel, Switzerland), or $10^{-6} \mathrm{M}$ tRA (Sigma). In a second series of experiments, medium was removed when cells reached subconfluence, and the cells were then incubated in DMEM supplemented with Monomed (a defined serum-free medium containing insulin, transferrin, sodium selenite, 2mercaptoethanol, 2-aminoethanol, sodium pyruvate, glutamine, and a BSA-oleic acid complex; Commonwealth Serum Laboratories, Melbourne, Australia) containing the above described agents. Vehicles $\left(0.1 \% \mathrm{BSA}\right.$ in $4 \mathrm{mM} \mathrm{HCl}$ for TGF- $\beta_{1}$ or $-\beta_{2}$, and dimethylsulfoxide [DMSO; Fluka] for tRA) were added to control cells at the same concentration as for treated cells. Medium was changed after three days, and cells were treated for six days.

\section{Cell proliferation and $[\mathrm{H} \mathrm{H}$-thymidine incorporation}

For evaluation of proliferation, SMCs were plated in DMEM supplemented with $10 \%$ FCS as described above and were counted after seven days using a haemocytometer. The results were calculated as the ratio of counted to seeded cells.

For $\left[{ }^{3} \mathrm{H}\right]$-thymidine incorporation, SMCs were plated at a density of 60 cells $/ \mathrm{mm}^{2}$ in DMEM supplemented with $10 \%$ FCS. At subconfluence, cells were synchronized for three days in DMEM containing Monomed. Fresh medium plus $10 \%$ FCS alone or medium containing one of the above-mentioned agents and $0.1 \mu \mathrm{Ci} / \mathrm{ml}\left[{ }^{3} \mathrm{H}\right]-$ thymidine (specific activity, $5 \mathrm{Ci} / \mathrm{mmol} \mathrm{I}^{-1}$; Amersham) was then added for 24 hours. $\left[{ }^{3} \mathrm{H}\right]$-thymidine incorporation was evaluated by trichloracetic acid and perchloric acid precipitation and counting in a liquid scintillation counter (Beckman Instruments, Irvine, CA).

\section{Cell migration}

For evaluation of migratory capacity, SMCs were plated at a density of 60 cells $/ \mathrm{mm}^{2}$ in DMEM in the presence of $10 \% \mathrm{FCS}$. Confluent cultures were scratched with a silicon-coated stick to obtain a $0.8 \mathrm{~mm}$-wide in vitro wound ${ }^{12}$ and photographed in phase contrast using a Zeiss Axiovert 35 photomicroscope (Carl Zeiss, Oberkochen, Germany). Fresh medium plus FCS alone or medium containing one of the above-described agents was added. After 24 hours, nuclear staining with propidium iodide $(0.05 \mathrm{mg} / \mathrm{ml}$; Fluka) was performed, and migrating cells invading the empty space were counted using a Zeiss Axiovert 35 photomicroscope (Carl Zeiss) and a KS400 interactive image-analysis system (Carl Zeiss Vision). Six randomly preselected fields (length $2.5 \mathrm{~mm}$ ) were analyzed per condition. Results were calculated as the total number of migrated cells per field.

\section{Immunohistochemical techniques}

Immunohistochemical stainings were performed on $4 \mu \mathrm{m}$-thick sections from formaldehyde-fixed paraffin-embedded blocks. Sections were deparaffinized and immersed in methanol containing $0.5 \% \mathrm{H}_{2} \mathrm{O}_{2}$ for 10 minutes. Sections were then incubated for 1 hour at room temperature with the following primary antibodies: 
mouse monoclonal $\operatorname{lgG}_{2 a}$ specific for $\alpha$-SMA at a dilution of $1 / 250^{446}$, mouse monoclonal $\operatorname{lgG}_{1}$ anti-desmin at a dilution of 1/10 (Clone D33; Dako, Copenhagen, Denmark), affinity-purified rabbit polyclonal IgG specific for SM-MHC at a dilution of $1 / 7^{447}$, and undiluted mouse monoclonal $\lg G_{1}$ specific for smoothelin ${ }^{23}$.

Immunoreactivity of SM-MHC and smoothelin staining was intensified by three microwave treatments for five minutes in citrate buffer $(10 \mathrm{mM}, \mathrm{pH} \mathrm{6.0)}$ before using the first antibody. Sections were incubated 30 minutes at room temperature with a secondary biotinylated goat anti-mouse or anti-rabbit antibody at a dilution of $1 / 250$ (Dako). The presence of the specific protein was evaluated by means of the streptavidin-biotin complex peroxidase method (Dako). Development of peroxidase activity was done with 3-amino-9-ethylcarbazole (Sigma). Slides were counterstained with haematoxylin, mounted in Eukit (Kindler, Freiburg, Germany), and observed using a Zeiss Axiophot photomicroscope (Carl Zeiss) equipped with a Plan-Neofluar 20x/0.50 objective. Pictures were acquired with a high-sensitivity Coolview color camera (Photonic Science, London, UK). Images were processed with Adobe Photoshop 5.0 (Adobe System, Mountain View, CA) and printed with a digital Fujifilm Pictrography 4000 printer (Fujifilm, Tokyo, Japan).

For immunofluorescence, SMCs were cultured in the presence of $10 \%$ FCS, and simple or double stainings were performed directly in the culture dishes or on SMCs cytocentrifuged on glass slides ${ }^{38}$. Cells were then fixed in 1) methanol for 5 minutes at $-20^{\circ} \mathrm{C}$ and stained with anti- $\alpha-S M A$, in 2) ethanol for 30 seconds at room temperature and stained with anti-SM-MHC or anti-desmin, or in 3) methanol/acetone $(1: 1, \mathrm{vol}: \mathrm{vol})$ for 5 minutes at $-20^{\circ} \mathrm{C}$ and stained with antismoothelin. Anti-mouse $\operatorname{lgG}_{2 \mathrm{a}}$, anti-mouse $\operatorname{lgG}_{1}$, and anti-rabbit $\operatorname{lgG}$ coupled to either fluorescein-isothiocyanate (FITC) or tetramethyl-rhodamine-isothiocyanate (TRITC) were used as secondary antibodies. Cell counts on cytocentrifuged cells were performed using an Axiophot photomicroscope (Carl Zeiss) at 40x magnification for at least three different fields for each sample. Each experiment was repeated at least three times. Photographs were taken with Tmax 400 film (Eastman Kodak) by using oil immersion Plan-Apochromat 40x/1.3 and 63x/1.4 objectives.

\section{Confocal laser scan microscopy (CLSM) analysis}

Cells were grown in DMEM supplemented with $10 \%$ FCS on Labtec slides (Miles Scientific, Naperville, IL), stimulated for three days with heparin at a concentration of $200 \mu \mathrm{g} / \mathrm{ml}$, fixed in methanol/acetone (1:1, vol:vol) for 5 minutes at $-20^{\circ} \mathrm{C}$, and stained with anti- $\alpha$-SMA, anti-vimentin, or anti-smoothelin, followed by the abovedescribed secondary antibodies.

Cell specimens were observed with a Zeiss confocal laser scan fluorescenceinverted microscope (LSM 410; Carl Zeiss) equipped with two lasers used simultaneously: a helium-neon laser (excitation wavelength at $543 \mathrm{~nm}$ ) and an argon laser (excitation wavelength at $488 \mathrm{~nm}$ ). The excitation spectra were separated by a dichroic beam splitter of $488 / 543 \mathrm{~nm}$, and the emission spectra of 
the two fluorochromes were separated by a $560 \mathrm{~nm}$ dichroic beam splitter. Two detectors were used in parallel and were preceded by a 590- to 610-nm (rhodamine channel) or a 510- to 525-nm (fluorescein channel) narrow-band barrier filter. The partial superposition of the emission spectra of the two fluorochromes was negligible ${ }^{448}$. Specimens were observed through an oil immersion Plan-Neofluar $63 \times / 1.4$ objective. Between 20 and 40 optical sections of $512 \times 512$ pixels separated by $0.25 \mu \mathrm{m}$ were performed in the z-axis. A three-dimensional image corresponding to the projection of all optical sections in one plane was reconstructed using the Imaris Program (Bitplane, Zurich, Switzerland) running on an Octane Silicon Graphics Workstation (Mountain View, CA). Colocalization of smoothelin and $\alpha-S M A$ or vimentin was evaluated by counting the number of pixels containing both stainings (yellow color) in all optical sections using colocalization software developed by Bitplane.

\section{Protein extraction, electrophoresis and Western blotting}

Freshly isolated cells and confluent culture dishes after trypsinization and cell samples were washed in phosphate buffered saline (PBS) supplemented with protease inhibitors: $10 \mathrm{mM}$ ethylenebis-(oxyethylenenitrilo)-tetra-acetic acid

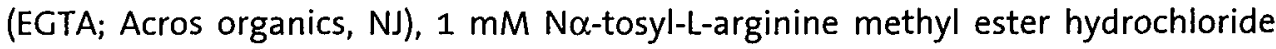
(TAME; Fluka), $1 \mathrm{mM}$ phenylmethanesulfonyl fluoride (PMSF; Fluka), $40 \mathrm{mg} / \mathrm{l}$ aprotinine (Trasylol; Bayer AG, Zurich, Switzerland), $0.5 \mathrm{mM}$ benzamidine (Sigma), 1 $\mathrm{mM}$ diisopropylfluorophosphate (DFP; Fluka). Cells were resuspended in sample buffer containing all of the aforementioned inhibitors plus $1 \%$ sodium dodecyl sulfate (SDS; Bio-Rad, Hercules, CA), 1\% dithiothreitol (DTT; ICN, Aurora, CA), 10\% glycerol (Fluka), $80 \mathrm{mM}$ Tris- $\mathrm{HCl}$ at pH 6.8 (Gibco), and bromophenol blue (Merck, Darmstadt, Germany), sonicated (Sonifier; Branson sonic power company, Danbury, $\mathrm{CT}$ ), and boiled for three minutes. Protein concentration was determined according to Bradford ${ }^{449}$.

Proteins were separated by sodium dodecyl sulfate-polyacrylamide gel electrophoresis (SDS-PAGE) on a $5 \%$ to $20 \%$ gradient gel and stained with Coomassie brilliant blue (R250; Fluka). For Western blotting, 2 to $20 \mu \mathrm{g}$ of total protein were electrophoresed and transferred to a nitrocellulose filter (Protran nitrocellulose; Schleicher and Schuel, Dassel, Germany). Filters were incubated with either anti- $\alpha$-SMA at a dilution of 1/500, anti-desmin at a dilution of $1 / 500$, or antismoothelin antibody at a dilution of $1 / 5$ followed by a goat anti-mouse lgG at a dilution of $1 / 10.000$, or anti-SM-MHC at a dilution of $1 / 100$ followed by a goat antirabbit IgG at a dilution of 1/10.000. Enhanced chemiluminescence was used for detection (Amersham life science, Little Chalfont, Buckinghamshire, UK). Signals were digitized by means of an Arcus II scanner (Agfa, Mortsel, Belgium) and analyzed using the Image Quant software (Image Quant Analysis; Molecular Dynamics, Sunnyvale, CA). Results are shown as the sum of pixel values of the area of each band. 
To separate the different MHC isoforms, samples of total protein were electrophoresed on $4 \%$ to $10 \%$ gradient gels containing $0.2 \% \quad \mathrm{~N}, \mathrm{~N}$ methylenebisacrylamide. Gels were run at $150 \mathrm{~V}$ for 20 hours and were subsequently stained with Coomassie brilliant blue. For Western blotting, $50 \mu \mathrm{g}$ of total protein were separated and transferred onto a nitrocellulose filter (Schleicher and Schuel), which was incubated with a rabbit polyclonal IgG specific for SM-MHC isoform 1 and 2 at a dilution of 1/1.000 (Biomedical Technologies Inc., Stoughton, $M A)$. This antibody does not recognize non-muscle isoform- $B^{170}$ (Robert $B$. Low, personal communication 1998). A goat anti-rabbit IgG at a dilution of 1/10.000 was used as secondary antibody. Enhanced chemiluminescence was used for detection (Amersham). Quantification of results was accomplished as described above.

\section{$\alpha-S M A C D N A$ probe, RNA extraction, and Northern blotting}

Since the sequence of the porcine $\alpha$-SMA mRNA is not known, we selected a conserved sequence among mouse, rat, rabbit, and human in the $3^{\prime}$-untranslated region. This region is specific for a given isoform ${ }^{450}$, but may not be species specific. Two primers selected at the extremities of this conserved sequence were used for polymerase chain reaction (PCR).

For practical reasons, PCR amplification was performed on an $\alpha$-SMA CDNA already cloned in a plasmid ${ }^{451}$ containing parts of the $3^{\prime} U T R$ region of the gene, yielding a DNA fragment of $121 \mathrm{bp}$. Two $\mu$ l of this plasmid were amplified during 30 cycles $\left(94^{\circ} \mathrm{C}\right.$ for 30 seconds, $55^{\circ} \mathrm{C}$ for 60 seconds and $72^{\circ} \mathrm{C}$ for 60 seconds). PCR was performed in $1 \times$ reaction buffer $(50 \mathrm{mM} \mathrm{KCl} ; 10 \mathrm{mM}$ Tris- $\mathrm{HCl}, \mathrm{pH} 9 ; 0.1 \%$ Triton X100) containing $25 \mathrm{pmol} / \mu \mathrm{l}$ sense and reverse primer, $25 \mathrm{mM} \mathrm{MgCl} 2$ (Promega), $200 \mu \mathrm{M}$ each dNTP (Promega), and 1 unit Taq DNA polymerase (Promega). All PCR reactions began with a hot start. PCR products were then electrophoresed on $2 \%$ to $4 \%$ agarose gels. DNA was stained with ethidium bromide (Sigma) and visualized in UV light. Elution and purification of specific DNA fragments on gels were performed with a Jetsorb gel extraction kit (Genomed, Research Triangle Park, NC) according to manufacturer information. Ligation and cloning of the specific insert were accomplished by using the PCR 2.1 vector (Invitrogen, San Diego, CA). Identity and orientation of the insert in the recombinant plasmid were confirmed by sequencing (T7 Sequencing Kit; Pharmacia Biotech, Duebendorf, Switzerland) using a sequencing gel electrophoresis apparatus (Model S2001; Gibco).

For yet unexplained reasons, direct extraction of total RNA from the media of coronary arteries resulted in an important degree of RNA degradation. Therefore, total RNA was extracted from freshly isolated cells of the media according to manufacturer's instructions like for confluent culture dishes. For Northern blot hybridization, 10 to $20 \mu \mathrm{g}$ of total RNA were denatured with glyoxal, separated by electrophoresis on a $1 \%$ agarose gel, and blotted on hybond $\mathrm{N}$-filters (Amersham). After UV fixation, filters were stained with methylene blue to verify correct loading and transfer. Filters were then processed for hybridization by random priming (Megaprime DNA labeling; Amersham) of specific CDNA probes for $\alpha-S M A$. 
Prehybridization and hybridization were performed at $50^{\circ} \mathrm{C}$ for 3 and 15 hours, respectively, in $5 x$ standard saline citrate (SSC), $5 x$ Denhardt's solution $(0.02 \%$ Ficoll, $0.02 \%$ polyvinylpyrrolidone, $0.02 \%$ bovine serum albumine), $0.1 \%$ SDS, and $100 \mu \mathrm{g} / \mathrm{ml}$ salmon sperm DNA. After hybridization, filters were washed twice for 15 minutes at $50^{\circ} \mathrm{C}$ in $4 \times \mathrm{SSC}$ and $0.1 \%$ SDS. They were then exposed to Kodak X-Omat SO-282 (Eastman Kodak) at $-70^{\circ} \mathrm{C}$ for 24 hours to three days. Autoradiographic signals were digitized and processed as described above. $\alpha$-SMA mRNA levels were calculated taking into account differences in loading between lanes as assessed by scanning the 185 band of the methylene blue-stained membrane.

\section{Statistical analysis}

Results are shown as mean \pm standard error of the mean. For statistical evaluation, the results were analyzed by the Student's t-test. Differences were considered statistically significant at values of $P<0.05$.

\section{Results}

\section{Expression of SMC differentiation markers in vivo}

The porcine LAD has a mean diameter of approximately $4 \mathrm{~mm}$ just after branching away from the left circumflex artery, similar to the corresponding human vessel. Van Gieson elastin staining showed the typical architecture of a muscular artery with interrupted elastic fibers within the media (figure 2.1a). Elastic fibers were abundant in the internal portion of the adventitia.
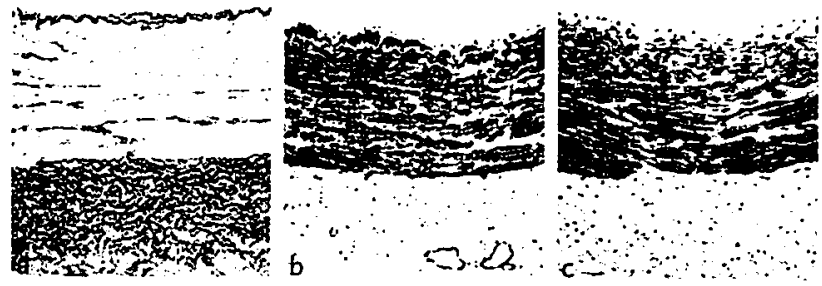

Figure 2.1 Immunohistochemistry of normal porcine coronary artery. (a) Van Gieson elastin staining showing collagen and elastin expression. Note the abundance of elastic fibers in the internal portion of the adventitia. Immunoperoxidase stainings for $\alpha$-SMA (b), SM-MHC (c), desmin (d), and smoothelin (e) show that nearly all SMCs in the media express these markers whereas

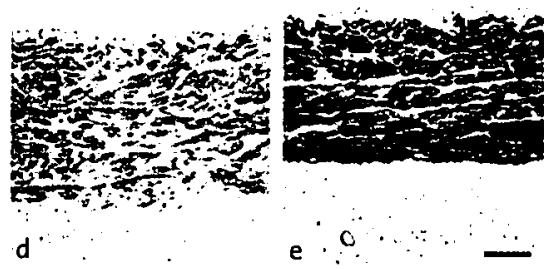
endothelial and adventitial cells do not (bar $=100 \mu \mathrm{m})$.

For immunohistochemical analysis of the coronary arteries, we chose a set of 4 well-accepted SMC differentiation markers: $\alpha$-SMA, desmin, SM-MHC, and 
smoothelin, a protein recently described in arterial tissue, probably the most specific marker of contractile SMCs ${ }^{23,79}$. Almost all SMCs in the media expressed $\alpha-$ SMA and SM-MHC (figure $2.1 \mathrm{~b}, \mathrm{c}$ ) whereas a small proportion of cells in the media appeared negative for desmin and smoothelin (figure 2.1d,e). Endothelial and adventitial cells were negative for all markers. All markers showed a similar staining distribution in proximal compared to distal segments of the same vessel (data not shown).
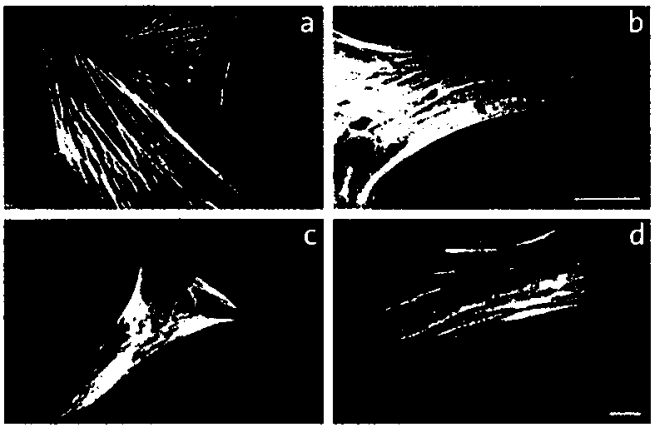

Figure 2.2 Morphological and cytoskeletal features of porcine coronary artery SMCs in vitro. Immunofluorescence stainings of porcine SMCs at the 5 th passage for $\alpha$-SMA (a), SM-MHC with typical interrupted distribution of the protein (b), desmin (c), and smoothelin (d). Note the stress fiber pattern of smoothelin distribution (Bar $=5$ $\mu \mathrm{m})$.

Features of SMCs in vitro

Enzymatic digestion was preferred to th. explant method because it allows to include a SMC population as representative as possible of the in vivo setting ${ }^{438}$. During primary culture and after subsequent passages, SMCs exhibited the typical spindle-shaped morphology with a hills-and-valleys pattern at confluence. SMCs displayed typical stress fibers after staining with anti- $\alpha$-SMA antibody (figure 2.2a). The SM-MHC staining demonstrated the classical interrupted distribution at the cell periphery (figure $2.2 \mathrm{~b}$ ). The anti-desmin antibody showed a pattern irradiating from the nucleus to the cell surface (figure 2.2c). The distribution of smoothelin has remained controversial up to now because this protein is not expressed spontaneously in cultured cells from conventional sources ${ }^{23,80}$. In our SMCs, a stress fiber-like organization of smoothelin was observed (figure $2.2 \mathrm{~d}$ ) with a strong intensity in the center of the cell and decreasing gradually toward the cell periphery.

CLSM analysis performed on cultured porcine SMCs showed that smoothelin was colocalized with $\alpha$-SMA (figure 2.3a,b) and not with vimentin (figure $2.3 c, d$ ). Colocalized pixels represented $85 \%$ of the total pixel number in the $\alpha$ SMA/smoothelin double staining and only $3 \%$ in the vimentin/smoothelin double staining.

Immunofluorescence staining performed on cytocentrifuged SMCs showed that in accordance with in vivo observations, almost all freshly isolated SMCs (0 hours) were positive for $\alpha$-SMA and SM-MHC (figure 2.4). About $80 \%$ of the cells were positive for desmin and smoothelin. After seven days of primary culture (PO), the proportion of positive cells decreased to about $70 \%$ for $\alpha$-SMA $(P<0.01)$, to $40 \%$ for 

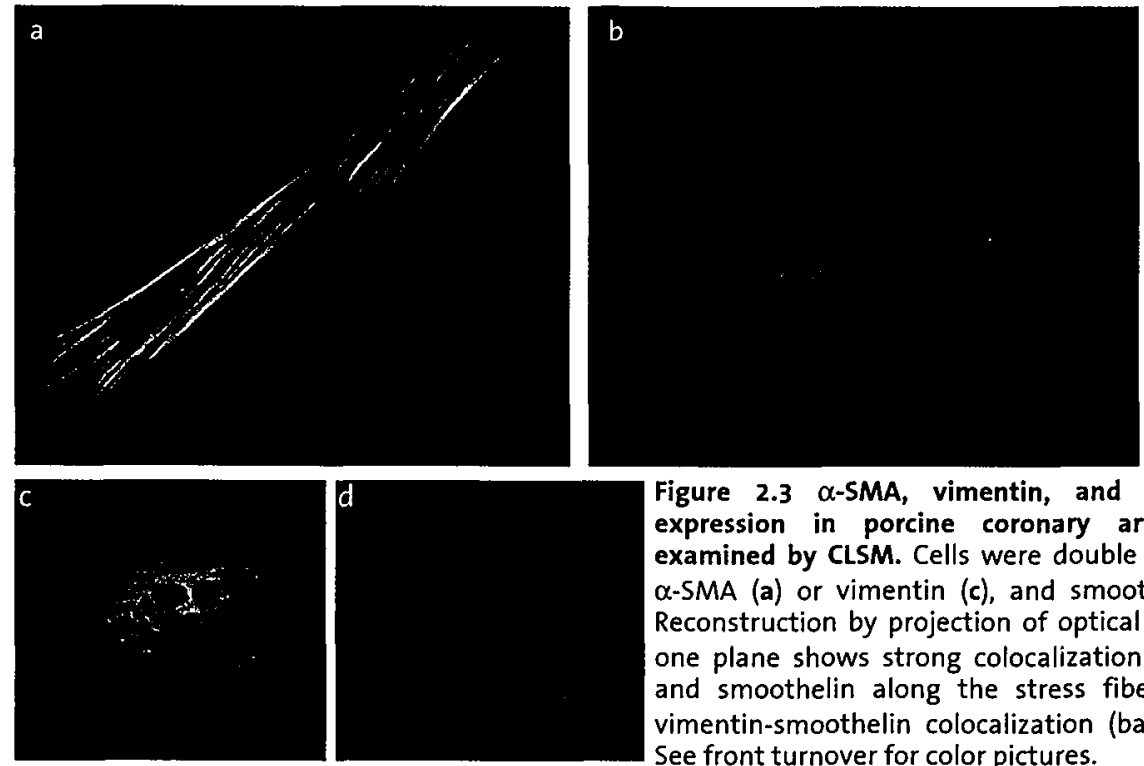

Figure 2.3 $\alpha$-SMA, vimentin, and smoothelin expression in porcine coronary artery SMCs examined by CLSM. Cells were double stained for $\alpha$-SMA (a) or vimentin (c), and smoothelin $(b, d)$. Reconstruction by projection of optical sections in one plane shows strong colocalization for $\alpha$-SMA and smoothelin along the stress fibers, but no vimentin-smoothelin colocalization (bar $=5 \mu \mathrm{m}$ ). See front turnover for color pictures.

desmin $(P<0.01)$, and to $35 \%$ for smoothelin $(P<0.01)$. The proportion of SM-MHC expressing cells remained unchanged; the intensity of staining, however, clearly decreased in about half of SM-MHC-positive SMCs; it remained at this level up to the fifth passage ( $\mathrm{P} 5$ ). After one passage ( $\left.\mathrm{P}_{1}\right)$, the percentage of $\alpha-\mathrm{SMA}$ - and smoothelin-positive SMCs remained at a level similar to that of primary culture whereas the percentage of desmin-positive SMCs decreased to about $30 \%$ ( $P 1$ versus $P O, P<0.05)$. At the fifth passage, the percentage of $\alpha$-SMA expressing cells increased to a level similar to that of $O$ hours ( $P 5$ versus $P 0$ and $P 1, P<0.01$ ). The proportion of desmin-positive cells also increased to a level similar to that of primary culture ( $P 5$ versus $P 1, P<0.05)$. The percentage of smoothelin-positive cells further decreased to about $15 \%$ ( $P 5$ versus $P 0$ and $P 1, P<0.01$ ). These results are different from those previously described for adult rat aortic SMCs in which percentages of $\alpha$-SMA-, SM-MHC-, and desmin-positive cells represent only approximately $80 \%, 45 \%$, and $30 \%$, respectively at Po and $70 \%, 3 \%$, and $0 \%$, respectively at $\mathrm{P} 5^{38,438,452}$.

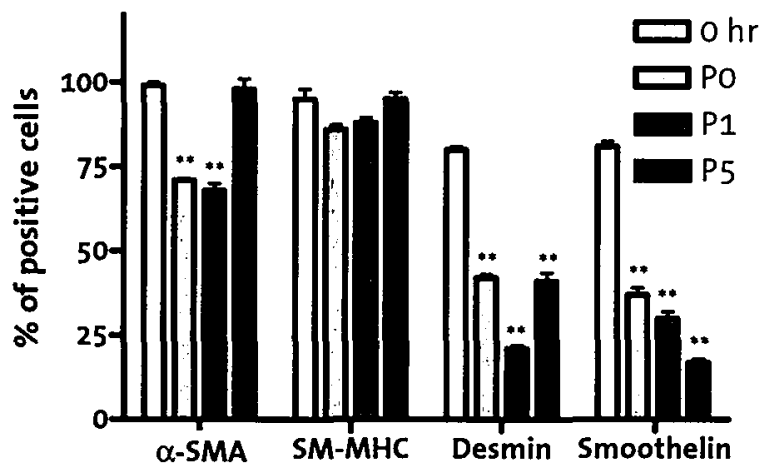

Figure 2.4 Percentage of porcine coronary artery SMCs expressing differentiation markers in vitro. Histogram showing the percentage of $\alpha-S M A, S M-M H C$, desmin, and smoothelin-positive cells just after enzymatic digestion (Ohr), in primary culture (P0), after 1 passage ( $P 1)$, and after 5 passages (P5). ("*: $P<0.01$; compared with $\mathrm{Ohr}$ ). 


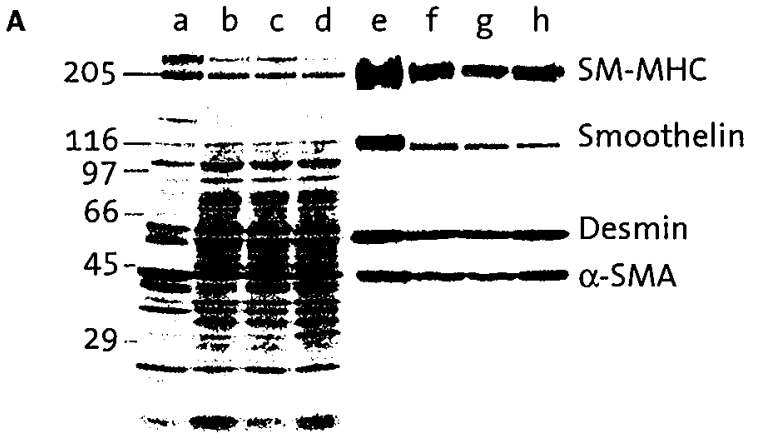

B

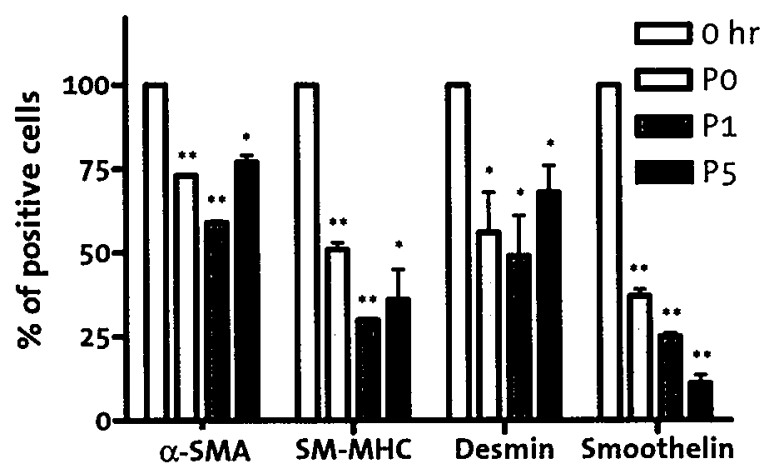

Figure 2.5 Expression of differentiation markers in porcine coronary artery SMCs in vitro (A) SDSPAGE of total protein extracts (a-d) and immunoblots (e-h) showing $\alpha$ SMA, SM-MHC, desmin, and smoothelin expression in SMCs just after enzymatic digestion $(a, e)$, in primary culture $(b, f)$, after 1 passage $(\mathbf{c}, \mathbf{g})$, and after 5 passages $(\mathrm{d}, \mathrm{h})$. (B) Densitometric scanning of immunoblots with values normalized to the pixel values measured in freshly isolated cells (o hr). $\alpha$-SMA-, SM-MHC-, and desmin content decreases in primary culture $(\mathrm{PO})$ to a level that is maintained at $P_{1}$ and $P_{5}$. Smoothelin content progressively decreases up to P5. ( $n=3$ experiments for each condition; ${ }^{*}=P<0.05, " *=P<0.01$; compared with $\mathrm{o} \mathrm{hr}$ ).

Immunoblotting results were in agreement with those of immunofluorescence studies (figure 2.5). In primary culture, the content of $\alpha$-SMA was decreased to about $80 \%(P<0.01)$, that of SM-MHC to $50 \%(P<0.01)$, that of desmin to $60 \%$ $(P<0.05)$, and that of smoothelin to $40 \%(P<0.01)$ compared with 0 hours. Values further decreased at the first passage, to about $60 \%$ for $\alpha$-SMA and $30 \%$ for $S M$ MHC $(P<0.05)$, whereas desmin and smoothelin values did not change. At the fifth passage, $\alpha$-SMA content increased and reached a value close to that of primary culture ( $P 5$ versus $P 1, P<0.05)$. The $S M-M H C$ and desmin contents were maintained at the levels observed at the first passage, i.e. $40 \%$ and $60 \%$ of the value at 0 hours, respectively ( $P 1$ versus $P 5$ not significant, $P 5$ versus 0 hours $P<0.05$ ). Smoothelin expression at the fifth passage represented $10 \%$ of the value at 0 hours and was significantly lower than in primary culture $(P<0.05)$. All studied proteins, including desmin and smoothelin, remained clearly detectable up to the ninth passage (data not shown). In rat aortic SMCs, the decrease of $\alpha-S M A, S M-M H C$, and desmin content during culture has been shown to be more pronounced than that observed in the pig model: at the 5 th passage $\alpha$-SMA content is only approximately $15 \%$ of the value at 0 hours, whereas SM-MHC and desmin proteins are not detectable $\mathrm{e}^{38,438,452}$. When separated by electrophoresis, the SM-MHC isoforms 1 and 2 exhibited a similar expression profile. In subculture, they represented about $50 \%$ of the value detected at 0 hours $(P<0.05$, data not shown). In passaged rat or rabbit 
arterial SMCs, no expression of SM-MHC 2 protein has been reported ${ }^{453-456}$. The expression of non-muscle $M H C$ isoform $B$ remained unchanged up to the fifth passage (data not shown). Confluence at the 5 th passage did not affect the level of $\alpha-S M A$ expression (data not shown), again different from previously reported rat data ${ }^{438,457}$. Thus, porcine coronary artery SMCs remain relatively well differentiated in culture. The expression of $\alpha$-SMA mRNA remained constant from freshly isolated cells up to the fifth passage ( $n=4$ experiments for each condition; data not shown). Moreover, the probe gave a specific signal also with rat and human SMC mRNA (data not shown).

\section{Effect of heparin, TGF- $\beta_{1}$ or $-\beta_{2}$, and tRA}

Several laboratories, including ours, have shown that heparin ${ }^{458,459}$, TGF- $\beta_{1}$ or $\beta_{2}{ }^{460,461}$, and, more recently, tRA ${ }^{24}$ are able to modulate cultured rat arterial SMC phenotype. Heparin as well as TGF- $\beta_{1}$ and $-\beta_{2}$ inhibit proliferation and induce differentiation of rat arterial SMCS, albeit through different mechanisms ${ }^{458-461}$. tRA is also capable of affecting differentiation of these cells ${ }^{24}$. To test whether porcine $S M C$ features can be modulated in culture by these agents, we exposed cells to heparin $(200 \mu \mathrm{g} / \mathrm{ml})$, TGF- $\beta_{1}(10 \mathrm{ng} / \mathrm{ml})$, TGF- $\beta_{2}(10 \mathrm{ng} / \mathrm{ml})$, or tRA $\left(10^{-6} \mathrm{M}\right)$ for six days. When compared with control cells, the proliferation of SMCs stimulated during seven days with $10 \%$ FCS was inhibited by $50 \%(P<0.01)$ in heparin-treated cells and by $30 \%(P<0.01)$ in cells growing in TGF- $\beta_{1}$ or $-\beta_{2}$-supplemented medium (figure 2.6a). tRA had no effect on cell proliferation. The $\left[{ }^{3} \mathrm{H}\right]$-thymidine incorporation rate, measured in synchronized SMCs 24 hours after addition of the different agents, was decreased to $40 \%(P<0.01)$ for heparin-treated cells and to only $60 \%, 75 \%$, and $80 \%(P<0.01)$ for cells treated with TGF- $\beta_{1}$, TGF $-\beta_{2}$, and tRA, respectively (figure 2.6b) when compared with control cells.

a

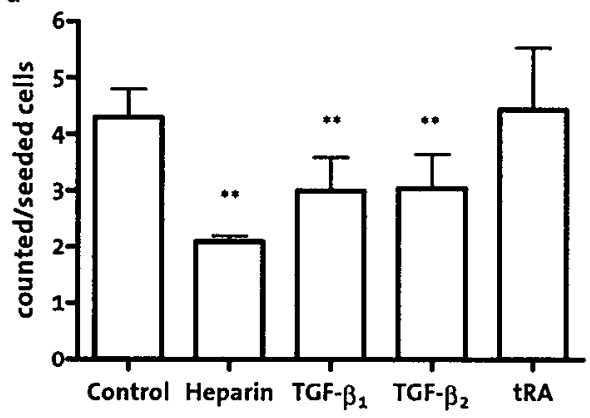

b

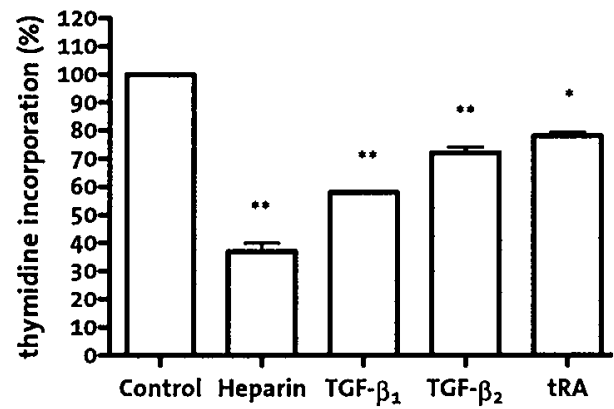

Figure 2.6 Effect of heparin, TGF- $\beta_{1}, T G F-\beta_{2}$, and tRA on proliferative activity of cultured porcine coronary artery SMCs. (a) Histogram showing the proliferation rate of SMCs treated during 7 days with $10 \%$ FCS alone (control) or supplemented with heparin $(200 \mu \mathrm{g} / \mathrm{ml})$, TGF- $\beta_{1}$ or $-\beta_{2}(10 \mathrm{ng} / \mathrm{ml})$, or tRA (10 M). (b) Histogram showing [ $\left.{ }^{3} \mathrm{H}\right]$-thymidine incorporation rate of synchronized SMCs treated for 24 hours with $10 \%$ FCS alone (control) or supplemented with one of the different agents described above. The $\left[{ }^{3} \mathrm{H}\right]$-thymidine incorporation (total $\mathrm{cpm}$ ) is normalized to the control value. Heparin is the most efficient agent to inhibit proliferation and $\left[{ }^{3} \mathrm{H}\right]$-thymidine incorporation of SMCs ( $n=5$ experiments for each condition; ${ }^{*}=P<0.05,{ }^{* *}=P<0.01$; compared with control). 
Migratory activity was assessed by measuring the capability of SMCs to invade an in vitro wound when treated with FCS alone or supplemented with one of the different agents (figure 2.7). After 24 hours of treatment, heparin decreased migration to about $40 \%$ and TGF- $\beta_{1}$ to about $60 \%$ when compared with control; TGF- $\beta_{2}$ and tRA had no significant effect under our conditions.

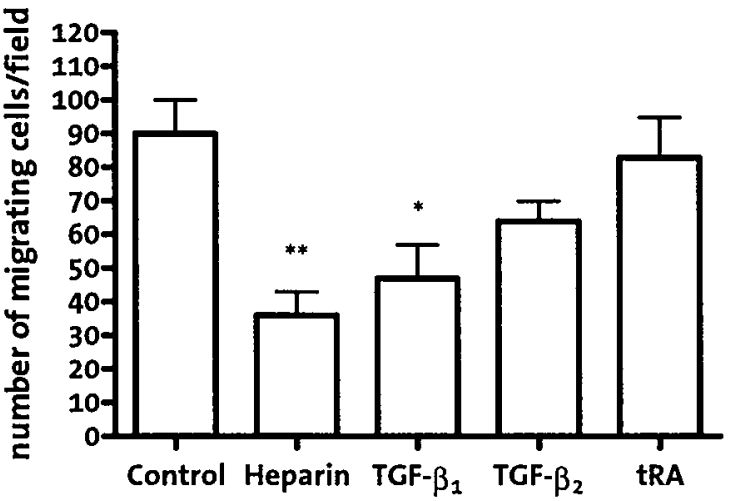

Figure 2.7 Effect of heparin, TGF- $\beta_{1}$, TGF$\beta_{2}$, and TRA on migratory activity of cultured porcine coronary artery SMCs. Histogram showing migratory capacity of cells cultured in the presence of $10 \%$ FCS without (control) or with heparin (200 $\mu \mathrm{g} / \mathrm{ml})$, TGF $-\beta_{1}$ or $-\beta_{2}(10 \mathrm{ng} / \mathrm{ml})$, or tRA $\left(10^{6} \mathrm{M}\right)$. Heparin is the strongest inhibitor of SMC migratory activity $(n=3$ experiments for each condition; ${ }^{\circ}: P<0.05$, **: $P<0.01$; compared with control).

Heparin increased the protein expression of SM-MHC 2, desmin, and smoothelin by 3.5-fold $(P<0.05)$, four-fold $(P<0.01)$, and three-fold $(P<0.05)$, respectively, whereas levels of $\alpha$-SMA and SM-MHC 1 were not significantly changed (figure 2.8). TGF- $\beta_{1}$ caused only a slight but significant decrease in SM-MHC $2(P<0.01)$ and a two-fold increase in the protein expression of desmin $(P<0.01)$. The expression of all other markers tested did not change after treatment with TGF- $\beta_{1}$. The only effect of TGF- $\beta_{2}$ was to cause a slight but significant decrease in SM-MHC $2(P<0.01)$. tRA only decreased the expression of desmin by $30 \%(P<0.01)$.

The effects of the different agents on SMCs were also tested in the absence of serum. As expected, SMCs did not grow in Monomed ${ }^{12}$. The migration of cells was inhibited by treatment with heparin and not with the other agents. Serum withdrawal itself had no effect on $\alpha$-SMA expression but increased the expression of SM-MHC 2 and to a lesser extent of desmin and smoothelin when compared with the $10 \%$ FCS condition, as assessed by Western blot analysis (data not shown). The expression of all differentiation markers was not affected by any of the abovementioned agents compared with the Monomed condition (data not shown).

\section{Discussion}

Our results show that SMCS cultured from porcine coronary artery retain a higher degree of differentiation than has been described in previous studies of SMCS cultured from animals such as the rat and rabbit, and that they maintain these properties even after several passages in culture. Thus, porcine cells represent a new 


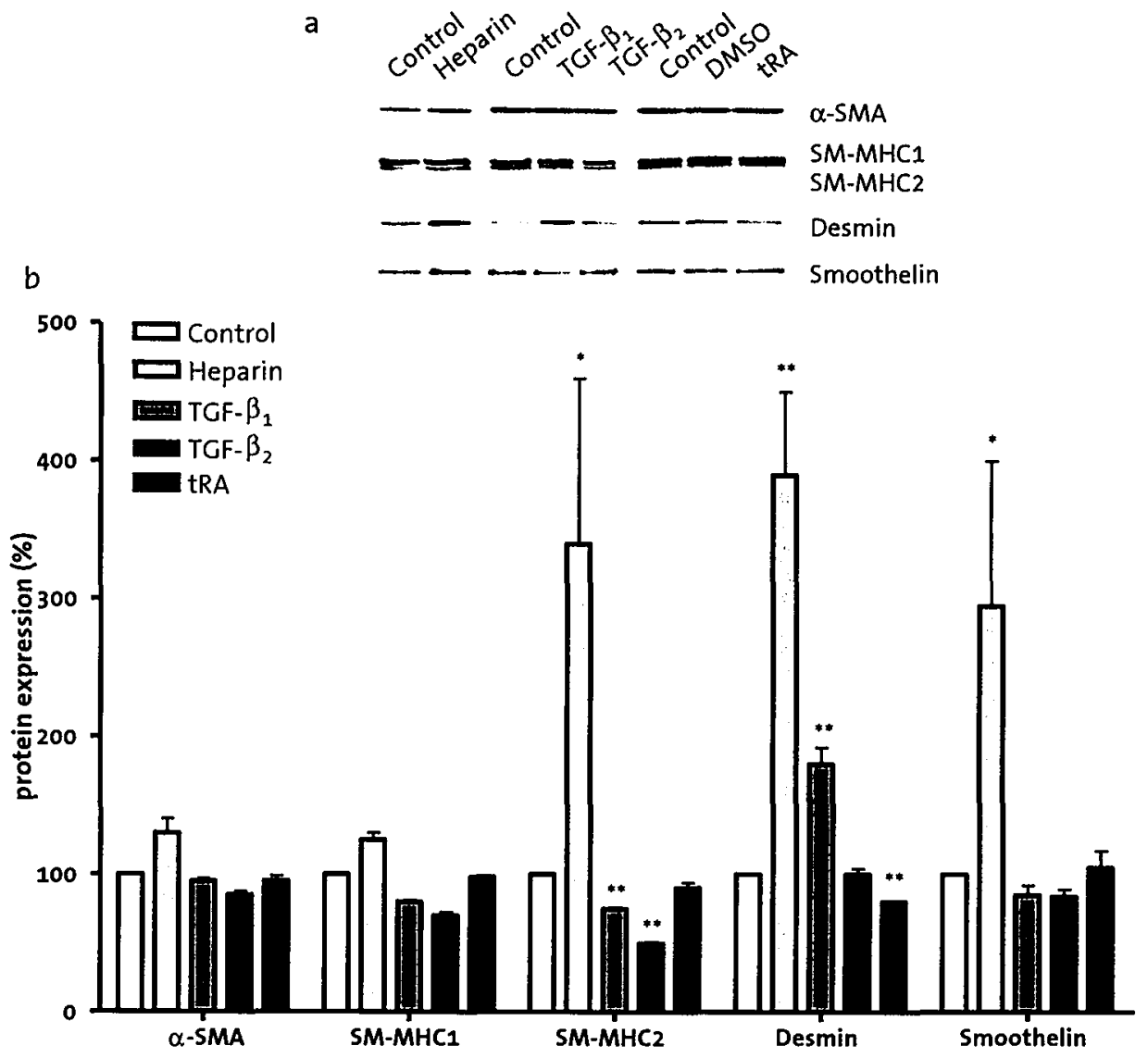

Figure 2.8 Effect of heparin, TGF- $\beta_{1}$, TGF- $\beta_{2}$, and tRA on protein expression in cultured porcine coronary artery SMCs. (a) Immunoblots showing protein content of cells cultured in the presence of $10 \%$ FCS without (control) or with heparin $(200 \mu \mathrm{g} / \mathrm{ml})$, TGF- $\beta_{1}$ or $-\beta_{2}(10 \mathrm{ng} / \mathrm{ml})$, or tRA $\left(10^{-6} \mathrm{M}\right)$ at the 5 th passage for $\alpha-5 M A, S M-M H C$ isoform 1 and 2, desmin, and smoothelin. (b) Histogram showing densitometric scanning of immunoblots with values normalized to the sum of the pixel values measured for control samples. Seven days of heparin treatment increases protein expression for SMMHC isoform 2, desmin, and smoothelin. TGF- $\beta_{1}$ treatment increases only the expression of desmin ( $n=3$ experiments for each condition; *: $P<0.05,{ }^{*}: P<0.01$; compared with control).

new model for examining the properties of well-differentiated SMCs, as well as regulation of the expression of differentiation markers such as SM-MHC and smoothelin and their isoforms. Porcine coronary arteries are very similar to human vessels in size and histology. Of all species examined to date, the pig is most similar to the human in its cardiovascular morphology and physiology, as well as its susceptibility to atheromatosis ${ }^{462,463}$. Human arterial SMCs also have been reported to replicate relatively more slowly and maintain a high degree of differentiation in terms of markers such as SM-MHC ${ }^{464}$ compared with rodent SMCs ${ }^{438}$. The fact that cultured porcine coronary artery SMCs also remain highly differentiated further suggests that the porcine culture model will be of importance in terms of 
improving our understanding of human vascular disease. It should be stressed that no well-established data on differentiation features of human vascular SMCs are present in the literature. The scarcity of data may be due to difficulties in obtaining standardized SMC populations from human donors.

Cytoskeletal proteins are well-accepted markers of the differentiation state of SMCs. Usually, a constellation of markers is necessary to assess SMC differentiation ${ }^{1}$, and we have followed the same criterion for cultured porcine coronary artery SMCs. As in the rat model, almost all porcine SMCs express $\alpha$-SMA and SM-MHC in vivo; but, differently from rat aortic SMCs where desmin is found only in half of the cells ${ }^{465}$, most porcine coronary SMCs express this marker. Smoothelin, which cannot be studied in the rat with the presently available antibodies, is present in practically all porcine SMCs, as previously described in human arteries ${ }^{23}$.

The expression of $\alpha-S M A$, desmin, and SM-MHC is maintained at much higher levels in porcine SMCs compared with rat and rabbit SMCs when using such criteria as number of positive cells and protein or mRNA contents ${ }^{38,439,452}$. In newborn rat arterial SMCS, the only situation in which SM-MHC is expressed at a relatively high level, only $43 \%$ of the cells are positive at the 5 th passage ${ }^{38}$. In the present report, we additionally have been able to discriminate between SM-MHC 1 and 2 protein expression. Both isoforms are markers of an advanced state of SMC differentiation, but SM-MHC 2 only appears at birth ${ }^{1,456,466}$. At the 5 th passage, more than $90 \%$ of the cells were positive for SM-MHC 1 and 2 whereas only a very small percentage has been shown to be positive in adult rat arterial SMCs ${ }^{467}$. Moreover, SM-MHC 2 protein was expressed in passaged porcine SMCs, while expression of this protein is strictly limited to primary culture in adult rat arterial $\mathrm{SMCs}^{453}$. It is noteworthy that about $50 \%$ of porcine coronary SMCs expressed desmin up to the 5th passage, whereas in rat aortic SMCs desmin is not detectable in similar conditions ${ }^{38,438}$. In general, the relationships between $\alpha$-SMA mRNA and protein expression correspond more closely in porcine coronary artery SMCs than they do for rat and rabbit ${ }^{439,452}$. This suggests a tighter coupling between transcription and translation than is found in the other species.

Smoothelin is a recently described cytoskeletal protein specific for SMCs. During development, it appears later than $\alpha$-SMA, desmin, and SM-MHC ${ }^{79}$. Smoothelin expression disappears during culture in all primary and passaged SMCs tested to date ${ }^{23}$. Porcine coronary SMCs however maintain expression of smoothelin protein up to the 9th passage. Two smoothelin isoforms have been described: a small isoform of $59 \mathrm{kDa}$ and a large isoform specific to vascular SMCs, to which the molecular mass of $94 \mathrm{kDa}$ had been assigned previously ${ }^{23,80,434}$. We have systematically tested cultured SMCs and arterial tissues, including those samples utilized for previous studies ${ }^{23,80,434}$, and conclude that the molecular mass of the large isoform is $115 \mathrm{kDa}$ in pigs, as assessed by electrophoretic migration.

The expression of smoothelin in cultured porcine SMCs allowed us to establish by CLSM that at least in cultured SMCs, smoothelin is colocalized with $\alpha-S M A$ and not 
with intermediate filaments. In transfection experiments, smoothelin displayed a stress fiber-like pattern, an intermediate filament-like pattern, or both, depending on the cell type used ${ }^{23}$. It is noteworthy in those regards that a putative actinbinding site has been suggested in the sequence of the smoothelin gene ${ }^{23}$. Further work using CLSM in tissues and assessing the binding capacity of smoothelin to $\alpha$ SMA will furnish more information on the possible interaction of the two proteins.

We have tested the effects of heparin, TGF- $\beta_{1}$ or $-\beta_{2}$, and tRA, which are known to modulate SMC differentiation, proliferation, and migration. Heparin, used at a concentration that has shown maximal effects on rat SMC proliferation ${ }^{459}$, inhibited proliferation and increased differentiation marker expression only in the presence of serum whereas migration was inhibited by heparin also in the absence of serum. Similar findings have been described for rat and rabbit SMCs ${ }^{459,468-472}$. Heparin did not affect the expression of $\alpha-S M A$ and SM-MHC 1, but increased the expression of SM-MHC 2, desmin, and smoothelin, suggesting that a low level of protein expression is a prerequisite for heparin activity.

Both TGF- $\beta$ isoforms reduced proliferation in porcine coronary SMCs, but migration was inhibited only by TGF- $\beta_{1}$. It is noteworthy that proliferation of rat SMCs can be inhibited or stimulated by TGF- $\beta_{1}$ according to the concentration used ${ }^{473-475}$. Both TGF- $\beta$ isoforms had no inducing effect on $\alpha-S M A$ or SM-MHC and in fact decreased expression of $S M-M H C$ 2. This finding is different from what Hautmann et al. have shown in rat arterial SMCs for both $\alpha$-SMA and SM-MHC ${ }^{186}$. However, these investigators used a lower dose of TGF- $\beta$ and different growth conditions. Interestingly, TGF- $\beta_{1}$ increased desmin expression in porcine SMCs, a marker that is rarely modulated in other models $s^{459}$.

tRA is considered to be a potential inducer of SMC differentiation ${ }^{169,196,476}$. However, proliferation, migration, and differentiation marker expression were not changed in porcine SMCs when treated with tRA. Nevertheless, tRA, similarly to heparin, TGF- $\beta_{1}$ and $-\beta_{2}$, reduced serum-stimulated $\left[{ }^{3} \mathrm{H}\right]$-thymidine incorporation. tRA inhibits rat arterial SMC proliferation ${ }^{199.201 .477}$, and $\left[{ }^{3} \mathrm{H}\right]$-thymidine incorporation is decreased only in SMC whole populations and clones with an epithelioid phenotype ${ }^{477}$. Moreover, IT development after endothelial injury is reduced in rats fed with a tRA-supplemented diet ${ }^{203.477}$. On the contrary, tRA stimulates migration of cultured rat SMCs as well as the expression of tissue-type plasminogen activator, the main enzyme responsible for the degradation of extracellular matrix in this cell type ${ }^{477,478}$. Interestingly, in cultured adult rat aortic SMCs ${ }^{477}$ and in the present model, $\alpha$-SMA expression was not affected by tRA. Taken together, these findings suggest that tRA acts mainly on poorly differentiated SMCs.

In conclusion, we have defined several biological and differentiation features of cultured porcine coronary artery SMCs. We have shown that they differ importantly from those established previously for rat and rabbit SMCs in terms of maintaining a more advanced degree of differentiation; in this respect, they appear to exhibit features reminiscent of those observed in human arterial SMCs. Our results indicate the potential value of these cells in the analysis of differentiation 
marker expression in SMCs. Porcine cells derived from the normal media or from the intimal thickening induced by angioplasty and/or stenting may provide further insight into the mechanisms involved in those processes as well as on the action of substances capable of influencing the biological behavior of SMCs in vitro and possibly in vivo.

\section{Acknowledgements}

This study was supported by the Swiss National Science Foundation, grant number. 31.50568.97. We are grateful to R.B. Low for critically reading the manuscript. We thank F. Gabbiani, P. Ropraz and A. Geinoz for their expert technical assistance, J.-C. Rumbeli and $\mathrm{E}$. Denkinger for photographic work, and $\mathrm{M}$. Vitali for help in typing the manuscript. 



\section{Smoothelin expression during chicken embryogenesis: detection of an embryonic isoform}

Marco de Ruiter*, Sander Rensen**, Gisela Coolen**, Beerend Hierck*, Maarten Bergwerff*, Wiel Debie**, Adriana Gittenberger-de Groot*, Guillaume van Eys**

- Department of Anatomy and Embryology, Leiden University Medical Center, Leiden, The Netherlands ** Department of Genetics and Cell Biology, Cardiovascular Research Institute Maastricht, Universiteit Maastricht, Maastricht, The Netherlands 


\section{Abstract}

Two isoforms of a novel smooth muscle cell (SMC)-specific cytoskeletal protein, smoothelin, have been described. In the adult chick, the $55 \mathrm{kDa}$ smoothelin-A is expressed in visceral SMCs, whereas the $120 \mathrm{kDa}$ smoothelin-B is the major product in vascular SMCs. The expression pattern of smoothelin was studied in chicken during embryogenesis and neonatally. Smoothelin-B was found in vascular SMCs from stage 20 onward. In visceral SMCs, smoothelin-B was present from stage 29 until hatching. Perinatally, a strong upregulation of smoothelin synthesis was observed in visceral tissues, coinciding with a switch to the A-isoform. Transient smoothelin synthesis was detected in the somites and the developing heart. Western blotting revealed a $62 \mathrm{kDa}$ smoothelin isoform in these tissues, designated smoothelin-C. Expression of the smoothelin isoforms seems to be strictly controlled with respect to cell type and developmental stage, and may be related to the mode of contraction of the different cells.

\section{Introduction}

The phenotype of SMCs varies, depending on their origin, location, or function, and can change during maturation and disease. SMCs express a combination of proteins characteristic of the degree of maturation ${ }^{1}$. SM-calponin, SM22 $\alpha$, heavycaldesmon (h-caldesmon), $\alpha$-smooth muscle actin ( $\alpha$-SMA), and smooth muscle myosin heavy chain have been described as markers of SMC lineages and differentiation stage. Some of these markers are specific for SMCs in the adult, but not in the embryo. SM-calponin, $S M 22 \alpha$, and $\alpha$-SMA are also expressed in embryonic skeletal and cardiac muscle cells ${ }^{121}$.

Smoothelin, a SMC-specific protein in adults, has been claimed to be useful to monitor SMC differentiation ${ }^{23}$. Although its function is not yet known, its expression has been found to be restricted to contracting $S M \mathrm{Ss}^{83}$. In human, two isoforms have been described: smoothelin-A, a $59 \mathrm{kDa}$ protein predominantly expressed in visceral and urogenital SMCs, and smoothelin-B, a $110 \mathrm{kDa}$ protein in vascular $5 \mathrm{MCs}^{80}$. Both isoforms are transcripts of one single gene $\mathrm{e}^{433}$ and appear to be the result of different transcriptional regulation.

We have studied the expression of smoothelin during chicken embryogenesis. Smoothelin was found in all muscle lineages. A new isoform was detected in striated muscle cell lineages, whereas smoothelin-B was shown to be the predominant isoform in SMCs. Around hatching, a shift to smoothelin-A was seen in visceral SMCs. This expression pattern provides clues for the function of smoothelins. 


\section{Materials and methods}

Fertilized White Leghorn eggs (2-18 days), neonatal chickens (1-7 days), and adult chickens were used. Smoothelin isoforms were detected immunohistochemically ${ }^{124}$ by the mouse monoclonal R4A antibody $\left(\operatorname{lgG}_{1}\right)^{23}$. Other monoclonal antibodies used in this study were HHF35 (anti-muscle actin; M635, Dako), 1A4 (anti- $\alpha$-SMA, M851, Dako), anti-SM-calponin (C6047, Sigma), and anti-heavy caldesmon (C6542, Sigma). Control sections of embryos of different stages of development incubated with only the rabbit-anti-mouse-peroxidase antibody (D0260, Dako) were completely negative (data not shown). Western blotting was performed as described previously ${ }^{80}$. In situ hybridization with DIG-labeled riboprobes of the 163 bp exon 20 Pstl-Smal fragment, both whole-mount and on sections, was performed according to instructions of the manufacturer (Boehringer).

\section{Cloning and characterization of chicken CDNA}

A lambda-ZAP cDNA library of embryonic (day 14) chicken heart tissue (Stratagene, La Jolla, CA, USA) was screened with a human smoothelin-A CDNA clone (GenBank accession number Z49989) according to the manufacturer's manual. Screening of the library was done by hybridization at $53^{\circ} \mathrm{C}$ in a $7 \%$ SDS/phosphate buffered solution. Positive clones were sequenced according to Sanger ${ }^{479}$. Searches for sequence homology were performed through GenBank/EMBL databases. 5'RACE and RT-PCR were performed on total RNA isolated from tissues of different embryonic stages by Trizol extraction (Life Technologies, Gaithersburg, MD).

\section{Results and discussion}

The chicken smoothelin cDNA was cloned and sequenced. Sequence homology between chicken on the one hand and mouse and human on the other was great (approximately $70 \%$ homology at the DNA level and $66 \%$ at the protein level; Genbank accession number AF272975). An alternative splice product (including exon 20) was found, but this appeared to have no physiological significance, because it was equally present in all smooth muscle tissues.

\section{Expression in non-SMCs}

Western blotting of embryonic tissues showed, in addition to smoothelin-A and $-B$, which are $55 \mathrm{kDa}$ and $120 \mathrm{kDa}$ in chicken respectively, an unknown $62 \mathrm{kDa}$ isoform (figure 3.1). Expression of this isoform, which we have designated smoothelin-C, was restricted to embryonic skeletal and cardiac muscle tissues. Sequence and 5'RACE analysis demonstrated that smoothelin- $C$ is a smoothelin-A variant. No differences in size were found between reverse transcriptase polymerase chain reaction (RT-PCR) products of the embryonic heart and skeletal muscle compared with adult gizzard and gut. This finding suggested that the increase in molecular 


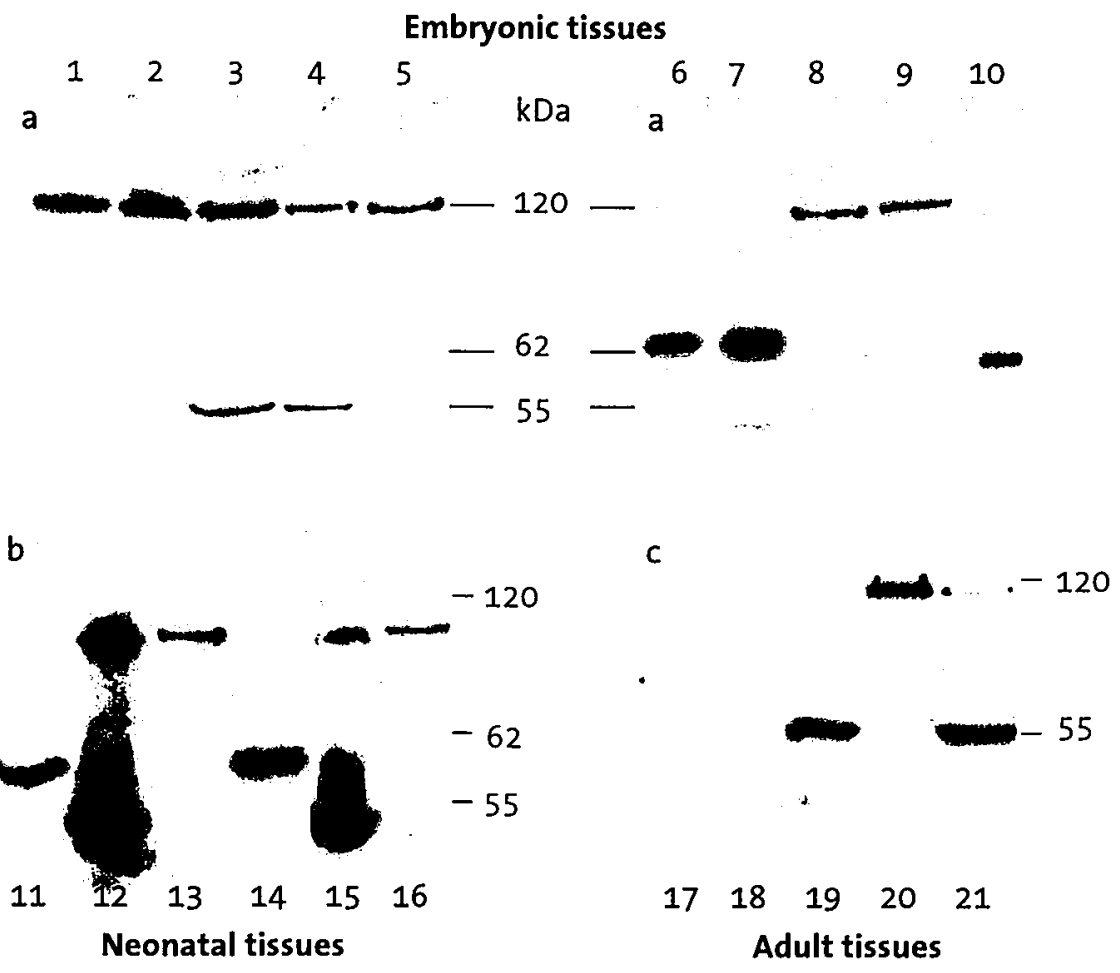

Figure 3.1 Western blot analysis of proteins extracted from embryonic stage $37 / 39$ (a; lane 1-10), neonatal 1 day ( $b$; lane 11-14), neonatal 7 days ( $b$; lane 15-16) and adult tissues ( $c$; lane 17-21). Lane (1) aorta descendens; (2) collection of muscular arteries; (3) stomach; (4) gut; (5) gizzard; (6) heart; (7) breast skeletal muscle; (8) aorta descendens; (9) collection of muscular arteries; (10) human colon (as reference); (11) skeletal muscle (breast); (12) gizzard; (13) aorta; (14) heart; (15) gizzard; (16) arteries; (17) heart; (18) skeletal muscle; (19) esophagus; (20) aorta; (21) gizzard. The faint $120 \mathrm{kDa}$ in lane 21 was smoothelin in the gizzard blood vessels, as confirmed by immunohistochemistry.

weight is due to posttranslational modifications such as glycosylation, as has been previously found in human smoothelin- $\mathrm{A}^{23}$.

Expression of smoothelin- $C$ in the somites (from stage 17) closely followed expression of $\alpha$-SMA (from stage 15). At stage 17, all somites except the four most distal ones expressed smoothelin. RNA transcripts of smoothelin were detected only in a defined dorsoventral band in the middle of the myotome, whereas in later stages the whole myotome stained for the protein (figure 3.2a-e). Although $\alpha$-SMA already had disappeared from these tissues at stage 27 , and had been replaced by skeletal-muscle specific actins, smoothelin transcripts and proteins were still abundant in the differentiated skeletal muscle cells at stage 37. Smoothelin levels decreased from stage 39 onward, but were still detectable even 7 days after hatching. No smoothelin has been found in adult skeletal tissues (figure 3.1).

The heart tube, with the exception of the outflow tract, also started to express the smoothelin-C isoform from stage 17 (figure 3.1, 3.2a). At stage 21, most of the myocardial mass is made up by trabeculae, which generate the largest part of the 

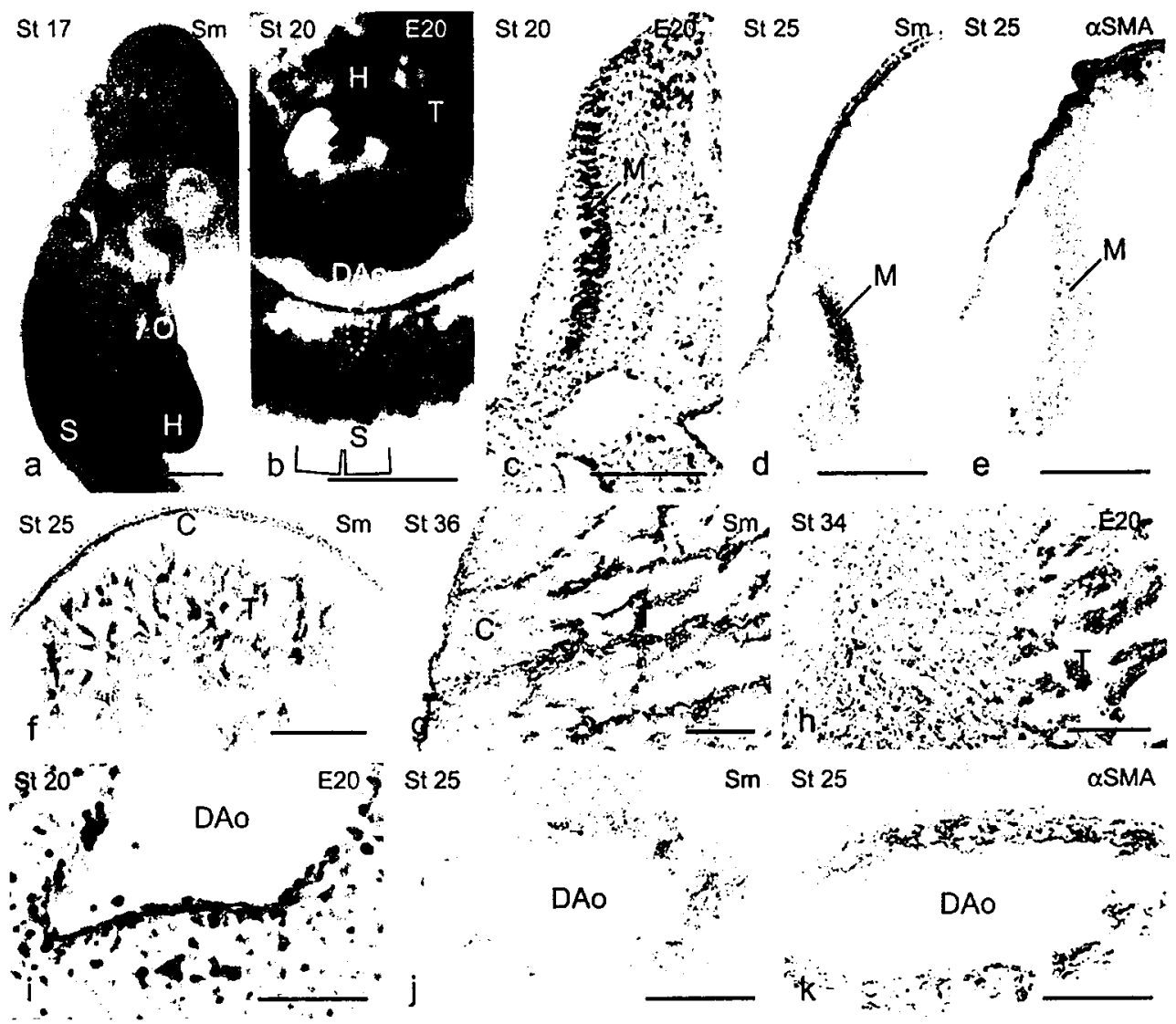

Sm

St 25

$\alpha$ SMA
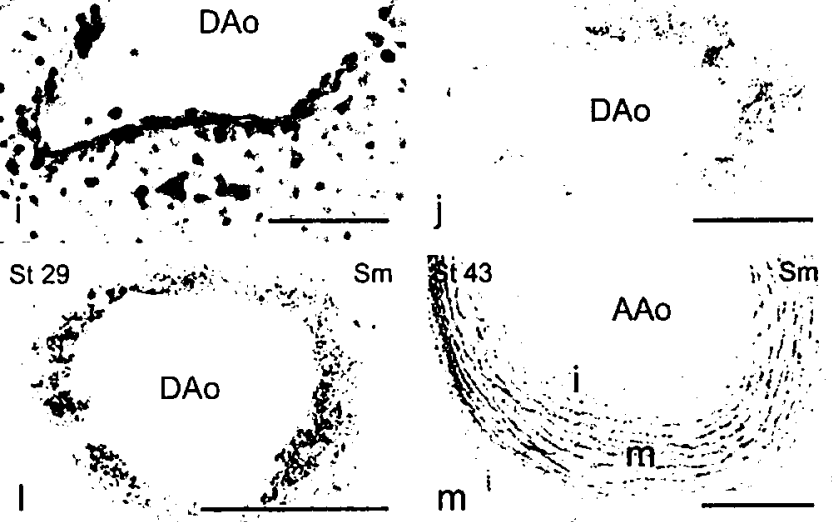

$\therefore$ कot

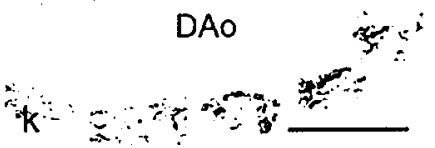

Figure 3.2 (a) Smoothelin expression $(5 \mathrm{~m})$ in the heart $(\mathrm{H})$ and somites $(\mathrm{S})$. The outflow tract of the heart (O) was negative. (b) Whole mount in situ hybridization (ISH) with an exon 20 probe detected smoothelin transcripts in the somites, descending aorta (Dao), and the trabeculae (T) of the heart (see also sections in (c) and (i)). (c) Smoothelin transcripts in the somites as detected by an exon 20 probe (embryo shown in (b)) were found only in the myotome $(M)$. (d,e) Adjacent sections incubated with antibodies against smoothelin and $\alpha$-SMA, respectively, showed specific expression in the myotomes of the $12^{\text {th }}$ cervical somite. ( $\left.f-h\right)$ Smoothelin expression in the heart disappeared from the compact myocardium (C) and became restricted to the trabeculae (T) at stage 36 . (h) ISH demonstrated transcription in the trabeculae. (i) Smoothelin transcripts in the primary SMCs of the descending aorta of the embryo shown in (b). (j-n) Smoothelin expression in descending and ascending aorta (Aao) at different stages of development. Smoothelin was confined to the differentiated actin-positive SMCs. At stage $43(\mathbf{m}, \mathbf{n})$, smoothelin was present in media (m), but not in the intima (i). (n) Confocal image of the ascending aorta displaying $\alpha$-SMA (dim) and smoothelin (bright). The vessel wall consisted of alternating layers of actin/smoothelin-positive and -negative (asterisk) cells. In contrast to actin-fibers, smoothelin was mainly distributed toward the cell membrane (arrowheads). See front turnover for color picture. $B$ ar in $I=50 \mu \mathrm{m}$; bar in $C-E, F, H, J-N=100 \mu \mathrm{m}$; bar in $B$ and $G=200 \mu \mathrm{m}$; bar in $A=500 \mu \mathrm{m}$. 
contractile force of the ventricle ${ }^{480}$. At stage 25 , the concentration of smoothelin in the trabeculae of the ventricles was higher than in the compact myocardium of the free ventricular wall (figure $3.2 \mathrm{f}$ ), and at stage 36 smoothelin was completely restricted to the trabeculae (figure $3.2 \mathrm{~g}, \mathrm{~h}$ ). The reduced expression in the compact free wall myocardium coincided with proliferation of striated heart muscle cell $s^{480}$. The trabecular cells at stage 36 also expressed other smooth muscle proteins like $\alpha$-SMA and SM-calponin (not shown). Neonatally, trabecular expression of smoothelin was no longer detectable. Thus, the expression pattern of smoothelin in heart and somites is similar to those reported for $\alpha$-SMA, SM22 $\alpha$, and SMcalponin, which expressions are transient and cease before bith ${ }^{20,69,481}$.

\section{Expression in SMCs}

In situ hybridization demonstrated the first smoothelin mRNAs in primary vascular SMCs of the descending aorta at stage 20 (figure $3.2 \mathrm{~b}, \mathrm{i}$ ). Like in the non-SMC tissues, smoothelin expression was preceded by $\alpha-S M A$, which was detectable at stage 16 . Immunohistochemically, smoothelin was not detected before stage 25 . At that stage, approximately three to four layers of primary SMCs expressed $\alpha-S M A$, whereas smoothelin was confined to the innermost two to three layers (figure $3.2 \mathrm{j}, \mathrm{k}$ ). During further development (figure 3.21), the number of smoothelin-positive layers gradually build up to reach six at stage 39 , corresponding with the number of actin-positive layers. The thin-walled branches of the aorta and pulmonary trunk were negative for smoothelin until stage 40 . In situ hybridizations and immunohistochemistry showed that vessels with a neural crest contribution (e.g., ascending aorta and aortic arch) contained a medial wall with alternating actin/smoothelin-positive and -negative SMC layers (figure $3.2 \mathrm{~m})^{124}$. Confocal imaging demonstrated that actin-negative cells never contain smoothelin and that in actin-positive SMCs smoothelin always colocalized with actin (figure 3.2n). Western blotting showed the B-isoform to be expressed in embryonic, neonatal, and adult vascular SMCs (figure 3.1).

Visceral smoothelin expression was first observed in the proventriculus (stage 29), followed by gizzard and gut (stage 31), and esophagus and bronchi (stage 36). Surprisingly, visceral SMCs synthesized the B-isoform before birth, as shown by Western blotting (figure 3.1). Immunohistochemical examination excluded that this finding was due to smoothelin-positive vascular SMCs in the embryonic tissues. Moreover, the blood vessels within the viscera did not contain smoothelin at that time.

Smoothelin concentration in visceral SMCs strongly increased perinatally, as deduced from immunomicroscopy and Western blots. In the last stages of embryogenesis smoothelin-B gave way to smoothelin-A, a process completed after hatching. No marked changes in the intracellular distribution of smoothelin and its colocalization with $\alpha$-SMA were observed during this B-to A-transition. It has been described that the myogenic properties of intestinal SMCs undergo a maturation process, which results in changed contractile patterns ${ }^{482}$. In the developing chicken, 
the increase of smoothelin-A coincides with the onset of more propulsive contractions in the alimentary tract. Thus, the B- to A-transition may be a part of this maturation process, which probably occurs in response to changing demands on the SMCs of the alimentary tract around hatching. A similar correlation between increase of force and of smoothelin-A concentration has been recently described for the uterus during pregnancy ${ }^{483}$. These correlations suggest that smoothelin-A plays a role in strong, propulsive smooth muscle contractions.

Although smoothelin shares several expression characteristics with other SMCspecific proteins such as SM-calponin and $S M 22 \alpha$, it stands apart from other actinassociated proteins by expression of a heart/somite-specific isoform and by the change in the expression pattern that occurs around hatching. The presence of specific isoforms in different muscle tissues may be correlated with the force which the SMCs have to exert, suggesting a function for smoothelin as a modulator of SMC contraction. In tone keeping or gently contracting tissues such as blood vessels and embryonic alimentary tract, the smoothelin-B isoforms will be synthesized. In relatively strongly contracting tissues such as adult alimentary tract or early embryonic heart, A/C-type smoothelins are expressed.

\section{Acknowledgements}

We are indebted to E. Timmer, F. Vlems, and H. van Straaten for their help in the initial phase of this study. This study was supported by the Netherlands Heart Foundation (grant number D96.018). 



\section{4}

\section{Expression of the smoothelin gene is mediated by alternative promoters}

Sander Rensen*, Victor Thijssen*, Carlie de Vries**, Pieter Doevendans ${ }^{* * *}$, Sevilla Detera-Wadleigh ${ }^{* * * *}$, Guillaume van Eys*

- Department of Genetics and Cell Biology, Cardiovascular Research Institute Maastricht, Universiteit Maastricht, Maastricht, The Netherlands

** Department of Biochemistry, University of Amsterdam, Amsterdam, The Netherlands *** Department of Cardiology, Heart Lung Center Utrecht, The Netherlands I Interuniversity Cardiology Institute, Utrecht, The Netherlands

"*** Laboratory of Molecular Genetics, NIDCH, NIH, Bethesda, USA

Based on: Cardiovasc Res. 2002; 55: 850-863 


\section{Abstract}

Two major isoforms of smoothelin have been reported, a $59 \mathrm{kDa}$ smoothelin-A in visceral smooth muscle cells, and a $110 \mathrm{kDa}$ smoothelin-B in vascular smooth muscle cells. The present study was undertaken to investigate the expression of these smoothelin isoforms in different smooth muscle tissues, and to determine how they are generated.

Western blotting with a new, well-defined, smoothelin antibody was applied to confirm the existence of two major smoothelin isoforms. Northern blotting, reverse transcriptase-polymerase chain reaction (RT-PCR), primer extension, and 5' rapid amplification of CDNA ends ( 5 'RACE) were performed to analyze the expression of these isoforms in human and mouse. Promoter reporter assays were carried out to establish the existence of a dual promoter system governing the expression pattern of the gene.

The antibody C6G confirmed the existence of two smoothelin proteins. Northern blotting showed that in vascular tissues, a larger smoothelin transcript is generated than in visceral tissues. The cDNA of this larger smoothelin-B was cloned. Computer analysis of the open reading frame suggested an $\alpha$-helical structure of 130 amino acids at the amino terminus of smoothelin- $B$. The smoothelin gene was cloned and sequenced. It comprises about $25 \mathrm{~kb}$ and contains 21 exons. The translational start of smoothelin-B is located in exon 2, whereas transcription and translation of the previously described smoothelin- $A$ starts inside exon 10. Smoothelin-A and $-B$ were demonstrated to be generated by two physically separated promoters. Splice variants within the calponin homology domain at the $3^{\prime}$ end of the gene were found for both isoforms.

We conclude that the two major smoothelin isoforms are generated from a single gene by a dual promoter system in a tissue specific manner. Further variation in the smoothelin proteins is achieved by alternative splicing of the exons coding for the calponin homology domain.

\section{Introduction}

Smooth muscle cells (SMCs) are characterized by the expression of SMC-specific marker proteins such as SM-calponin, SM22 $\alpha, \alpha$-smooth muscle actin ( $\alpha$-SMA), smooth muscle myosin heavy chain isoforms (SM-MHC), and smoothelin. Despite the shared expression of these proteins, SMCs are heterogeneous in many respects. This heterogeneity is reflected in differences in morphology, physiology, and protein expression profiles of SMCs ${ }^{388,465,484}$. Accordingly, SMCs can be divided into sub-populations with a synthetic or a contractile phenotype ${ }^{13,388,439}$. Moreover, SMCs of the visceral organs, digestive tract, and the reproductive system differ from the SMCs found in the blood vessel ${ }^{485.486}$. Some of these variations are related to differences in embryological origin. SMCs that line the digestive tract and ducts 
associated with the gut, as well as SMCs around the bladder, trachea, and bronchi are all derived from the lateral mesoderm ${ }^{487}$. In contrast, the majority of the vascular SMCs is considered to be either locally recruited from the primordium, pro-epicardial organ, or neural crest after invasion by endothelial cells, or from circulating progenitor cells ${ }^{488,489}$. Trans-differentiation of endothelial cells to SMCs has also been reported ${ }^{132}$.

SMC-specific proteins can be used as markers of development or differentiation if their expression is specific for a particular phenotype or stage of SMCs, or if isoforms are characteristic of specific SMC lineages. Smoothelin has both properties. It is a marker protein for the end-stage of contractile SMC differentiation ${ }^{23,79,132}$. Furthermore, the two adult isoforms of smoothelin are expressed in a tissue specific manner: a $59 \mathrm{kDa}$ isoform, smoothelin-A, in visceral and urogenital tissues, and a $110 \mathrm{kDa}$ isoform, smoothelin- $B$, in vascular tissues (previously described as $94 \mathrm{kDa}$ protein) ${ }^{80,434}$. Transient synthesis of a third smoothelin isoform has been detected in embryonic striated muscle cells in chicken ${ }^{82}$.

SMCs can also be grouped according to the nature of their contraction. Tonic and phasic SMCs can be distinguished. Tonic SMCs, which are primarily found in blood vessels, have a slow rate of force activation and relaxation, low maximum speeds of shortening, and good force maintenance. Phasic SMCs, in contrast, show a high rate of force activation and relaxation, high maximum speeds of shortening, and poor force maintenance ${ }^{402}$. The latter are mainly found in SMCS of the gastrointestinal tract. These physiological differences in distinct SMC populations are reflected in proteins that make up the cyto-architectural structures of the $S M C s^{6,388}$. Studies in chicken embryos have revealed that during development, smoothelin-B is not only found in vascular, but also in visceral SMCs of the digestive tract. When the embryonic visceral SMCs change their contractile profile from tonic to phasic (around hatching), smoothelin-B is replaced by the smoothelin-A isoform ${ }^{82}$. This expression pattern not only makes smoothelin a valuable marker for SMC differentiation, but is also indicative of a function of smoothelin in SMC contraction.

Previously, we have demonstrated that smoothelin is encoded by a single copy gene, located on chromosome 22q12.3, and we and others have provided a rough map of the structure of the gene ${ }^{433,490,491}$. In this study, Northern blotting proved that there are two transcripts mediating the generation of the two major smoothelin isoforms. The cloning and characterization of vascular SMC-specific smoothelin-B CDNA and the smoothelin gene are described. Alternative splicing was found to introduce variations in the calponin homology domain ( $\mathrm{CH}$ domain) of the smoothelins, which probably mediates actin-binding. Promoter reporter assays showed that alternative promoters are used to produce smoothelin-A or smoothelin-B mRNA. 


\section{Materials and methods}

\section{Tissue samples and cell culture}

Human primary SMCs were obtained from iliac artery by enzymatic dispersion (collagenase/elastinase/soybean trypsin inhibitor: Worthington Biochem. Corp. Lakewood, NJ). Cells were cultured in Opti-MEM I Reduced Serum Medium supplemented with $15 \%$ fetal calf serum (Life Technologies Inc., Paisley, Scotland). Human tissues (umbilical cord, iliac artery, uterine artery, aorta, uterus, gut, colon, ileum, stomach, skeletal muscle, liver, brain, heart) and mouse tissues (uterus, prostate, lung, esophagus, stomach, colon, iliac artery, carotid artery, aorta, and heart) were isolated, immediately frozen, and stored at $-80^{\circ} \mathrm{C}$ until use. The use of human and animal tissues was approved by the ethical board of the university. Cells and tissues were screened for the presence of smoothelin by indirect fluorescent antibody techniques. Procedures were performed as previously described $^{23}$.

\section{Antibody generation}

To generate a mouse monoclonal antibody directed against human smoothelin, mice were immunized with recombinant human smoothelin-A. The fusion procedure and cloning of the hybridomas were performed according to standard protocols ${ }^{492}$. Isotyping of the monoclonal was done with the Mouse Mab Isotyping kit according to the manufacturer's instructions (Life Technologies). Smooth muscle specificity of the monoclonal was assessed by screening cells and tissues by Western blotting and indirect fluorescence staining as previously described ${ }^{23}$. The epitope recognized by the antibody was mapped using deletion expression constructs of the smoothelin-A cDNA. Several fragments were cloned in frame in the pRSET vector (figure 4.1a), and proteins were produced in Escherichia coli of the M15 strain and analyzed on Western blot.

\section{Protein gel electrophoresis and Western blotting}

Cryostat sections (20 per sample, $10 \mu \mathrm{m}$ ) of freshly frozen tissues were collected, washed with phosphate buffered saline (PBS), and centrifuged for $5 \mathrm{~min}$ at $12.000 \times g$. After centrifugation, the pellet was subjected to a Triton $X-100$ extraction step. Cells were resuspended in $1 \%$ Triton X-100, $5 \mathrm{mM}$ ethylenediaminotetraacetic acid disodium salt dihydrate (Merck, Darmstadt, Germany), 0.4 $\mathrm{mM}$ phenylmethylsulfonyl fluoride (Merck) in PBS, pH 7.4, and extracted for 5 min on ice. After centrifugation for $5 \mathrm{~min}$ at $12.000 \times \mathrm{g}$, the pellet was washed in $1 \mathrm{ml}$ PBS. After a final centrifugation step (5 min, 12.000xg), the preparation was dissolved by boiling for $4 \mathrm{~min}$ in sample buffer ${ }^{493}$, with $2.3 \%$ sodium dodecylsulfate (SDS) and $5 \% \beta$-mercaptoethanol (Bio-Rad Laboratories, Hercules, CA).

For SDS-polyacrylamide gel electrophoresis (SDS-PAGE) on a Mini Protean II Electrophoresis Cell (Bio-Rad Laboratories), 7.5-10\% polyacrylamide slab gels containing $0.1 \%$ SDS were used ${ }^{493}$. After electrophoretic separation, the proteins 
were stained with Page Blue 83 (BDH Chemicals Ltd., Poole, England) or subjected to Western blotting on a nitrocellulose membrane (Schleicher \& Schüll, BA85: Dassel, Germany). The monoclonal antibody $\mathrm{C6G}$, directed against smoothelin, was used at a 1:5 dilution. Antigen-antibody interaction was visualized by chemiluminescence (ECL-kit, Amersham, Buckinghamshire, UK).

Smoothelin expression analysis by Northern blot and RT-PCR

Total RNA of vascular tissues (umbilical cord artery, aorta, primary cell cultures of iliac artery) and non-vascular tissues (gut, colon, stomach, skeletal muscle) was extracted by $\mathrm{LiCl}^{494}$. Ten $\mu \mathrm{g}$ of total RNA were separated on a $1 \%$ agarose formaldehyde denaturing ge ${ }^{494}$. RNA was transferred to nitrocellulose (S\&S BA83), and hybridized to smoothelin CDNA probes according to standard procedures. Probes were ${ }^{32} \mathrm{P}$-labeled by random priming (Life Technologies) ${ }^{495}$. Filters were washed in decreasing SSC ( $\mathrm{NaCl} / \mathrm{NaCitrate)}$ concentrations with a final concentration of $0.1 \times \mathrm{SSC} / 0.1 \%$ SDS.

Alternative splicing was investigated by RT-PCR. Five $\mu \mathrm{g}$ of total vascular RNA extracted from mouse tissues were subjected to oligo(d)T-primed reverse transcription. Sets of primers that covered the smoothelin-A CDNA were used for $P C R$. Length of the PCR-generated fragments was compared with those generated by PCR of the smoothelin-A cDNA. Expression of detected alternative splice variants (all located in the $\mathrm{CH}$ domain) was further investigated by RT-PCR with the following primers: forward 5'GTCGACATCCAGAACTTCCTCC; reverse $5^{\prime C G C A G G T G G T T G T A C A G C G A . ~ T h e ~ P C R ~ c o n d i t i o n s ~ a p p l i e d ~ w e r e: ~} 2 \mathrm{~min}$ initial denaturation at $94^{\circ} \mathrm{C}, 30 \mathrm{sec}$ at $94^{\circ} \mathrm{C}, 30 \mathrm{sec}$ at $54^{\circ} \mathrm{C}, 30 \mathrm{sec}$ at $72^{\circ} \mathrm{C}(35 \mathrm{cycles})$, with a final extension of 5 min using an MWG AG Biotech Primus 96plus thermal cycler. PCR products were analyzed on a $2 \%$ agarose gel.

\section{Cloning of the human CDNA coding for smoothelin-B}

RNA was isolated from human iliac artery smooth muscle by Trizol extraction according to the manufacturer's instructions. Concentration and quality of RNA was evaluated by Northern blot analysis using a ${ }^{32} \mathrm{P}$-labeled smoothelin-A CDNA ${ }^{495}$. $5^{\prime} R A C E$ was applied to investigate the possibility of a $5^{\prime}$ extension of the smoothelin-A mRNA. Based on sequences of the $5^{\prime}$ part of the smoothelin-A CDNA, the following primers were used in a 5'RACE system (Life Technologies): 5'GCATGGCTGAAGCTGGTGAC and 5'GTCAATGTGGTCTTCATACTG. PCR fragments were generated and cloned. Colonies were screened with a genomic fragment containing the sequence from -200 to +60 of the smoothelin-A cDNA. Clones containing CDNA inserts were mapped by restriction digestion, and sequenced. Sequence comparison and structural analysis of the putative protein were carried out using BLAST algorithms ${ }^{496}$ and the protein structure program PHD $^{497,498}$.

Cloning and analysis of the human smoothelin gene

Approximately $10^{5}$ clones of a human placental cosmid genomic library 
(Stratagene, La Jolla, CA) were screened with a ${ }^{32} \mathrm{P}$-labeled smoothelin-A CDNA probe. Two positive clones were purified to homogeneity. One of the clones contained the complete coding region of the smoothelin gene. In addition, $6 \mathrm{~kb} \mathrm{5}$ flanking sequence was present. This clone was subcloned into pUC19 and exoncontaining subclones were identified by colony hybridization with cDNA fragments. A complete restriction map of the cosmid clone was constructed, and exons and their flanking regions were sequenced. Sequencing was performed according to Sanger et al. ${ }^{479}$ using an Applied Biosystems AmpliTaq cycling kit for automatic sequencing in an Applied Biosystems 310 (Perkin Elmer, Norwalk). Searches for sequence homology were performed through GenBank/EMBL databases. Intronic sequences were screened with HEXON software, which allows detection of potential additional exons ${ }^{499}$.

\section{Primer extension analysis}

The smoothelin-A transcription start site was determined by primer extension using a primer extension system from Promega (Madison, WI). Briefly, $10 \mu \mathrm{g}$ RNA extracted from human ileum with Trizol (Life Technologies) according to the manufacturer's instructions were reverse transcribed with Superscript II Reverse Transcriptase (Life Technologies) for $1 \mathrm{hr}$ at $42^{\circ} \mathrm{C}$ using the following $\left[\gamma^{-32} \mathrm{P}\right]$-endlabeled primer: GTGAATGTGGTCTTCATACTC. Annealing of the primer was done at $60^{\circ} \mathrm{C}$ for $1 \mathrm{hr}$. Primer extension products were analyzed on a gel containing $8 \%$ acrylamide, $7 \mathrm{M}$ urea, and $1 \times$ TBE buffer. Labeled $\varphi \times 174$ HinfI DNA markers were loaded onto the gel to determine the size of the generated fragments.

\section{Smoothelin promoter reporter assays}

To investigate the putative promoters of the smoothelin gene, smoothelin reporter constructs were made based on transcription start sites as determined by $5^{\prime} R A C E$ and primer extension assays (figure 4.6). Two smoothelin-A promoter fragments containing either 1613 or $370 \mathrm{bp}$ of the 5' flanking region, exon 10, and $60 \mathrm{bp}$ of intron 10 were cloned into the pGL3-basic vector (Promega) using Sstl and HindIII sites. The $1613 \mathrm{bp}$ fragment was subcloned into the $\mathrm{p} \beta \mathrm{Cal}$-basic vector (Clontech Laboratories inc., Palo Alto, CA) using Sphl sites.

Five smoothelin-B promoter fragments of increasing length were cloned into the PGL3-basic vector. The fragment sizes varied from 84 to 3372 bp of the region upstream of the smoothelin-B transcription start site. The first smoothelin-B exon and $28 \mathrm{bp}$ of the first intron were included. The promoter constructs were transfected into PAC1 ${ }^{245}, \mathrm{CHO}$, and Hela cells using Fugene-6 (Roche, Indianapolis, IN) according to the manufacturer's protocol. Analysis of luciferase expression was performed 2 days after transfection with a luciferase assay system according to the provided instructions (Promega) in a Biocounter M1500 luminometer (Lumac, Landgraaf, The Netherlands). Measurements were performed for 10 seconds after a 2 second delay. Co-transfection of a TK-promoter driven renilla luciferase vector for internal normalization influenced the behavior of the smoothelin promoter 
constructs tested, as previously described for $\alpha$-SMA promoter assays ${ }^{500}$. Instead, luciferase activity of a control PGL3-promoter vector containing an SV4O promoter upstream of the luciferase gene was used to correct for variations, after subtracting the baseline activity of the pCL3-basic vector. Variation in lysate recovery was corrected for by determining the protein concentration of the samples. All constructs were transfected in duplicate in three independent experiments. Cells transfected with the $\beta$-galactosidase reporter construct were fixed with $0.25 \%$ glutaraldehyde after 52 hours. Staining solution containing 0.1 mg X-Gal was applied for 18 hours at $37^{\circ} \mathrm{C}$.

\section{Results}

Application of the novel anti-smoothelin monoclonal antibody $\mathrm{C} 6 \mathrm{C}$

A monoclonal antibody, $\mathrm{C} 6 \mathrm{G}$, was generated against recombinant human smoothelin-A. The antibody of the $\lg G_{1}$ subclass reacted specifically with smooth muscle tissue. The epitope of the antibody, which recognizes both smoothelin-A and smoothelin- $B$, is located within a stretch of 30 amino acids in exon 11 (amino acids 56-86 of smoothelin-A, 512-542 of smoothelin-B; see figure 4.1a). Western blotting showed that the principal product recognized by $C 6 \mathrm{C}$ in visceral smooth muscle tissues was the $59 \mathrm{kDa}$ smoothelin-A, whereas in vascular tissues the major product identified was the $110 \mathrm{kDa}$ smoothelin-B (figure $4.1 \mathrm{~b}$ ). In non-smooth muscle tissues like liver, brain, or heart, no immunoreactive product was detected. C6G reacted with smooth muscle tissues of human, goat, and rabbit, but not with dog, pig, and mouse. C6G reacted relatively strongly with smoothelin-B compared with the previously described R4A antibody.

Cloning and characterization of human smoothelin-B CDNAs

Northern blotting analysis of cultured SMCs of human iliac artery, aorta, and umbilical cord artery revealed a transcript of $\sim 3000$ nt hybridizing with the smoothelin-A CDNA, whereas in visceral smooth muscle tissues the major hybridizing transcript was only about 1600 nt (figure 4.2). The identification of two smoothelin isoforms on Northern and Western blots, while only one smoothelin gene had been found, suggested aiternative splicing of the smoothelin gene $e^{80,433}$. An RT-PCR screen of RNA extracted from vascular tissues did not reveal an alternative splicing corresponding with the difference in size between the two smoothelin transcripts. Hence, a 5 ' extension of the smoothelin-A mRNA was investigated by a 5 ' RACE procedure using RNA of a primary culture of human iliac artery SMCs. A discrete band of $1700 \mathrm{bp}$ was generated in three independent experiments. This product was cloned and sequenced. Sequences of several clones were aligned and compared with the sequence of the smoothelin gene (see below). The 3000 nt transcript in iliac artery SMC cultures and vascular tissues hybridized with the 5' RACE fragment. In visceral tissues, hybridization of the 5'RACE product 
a

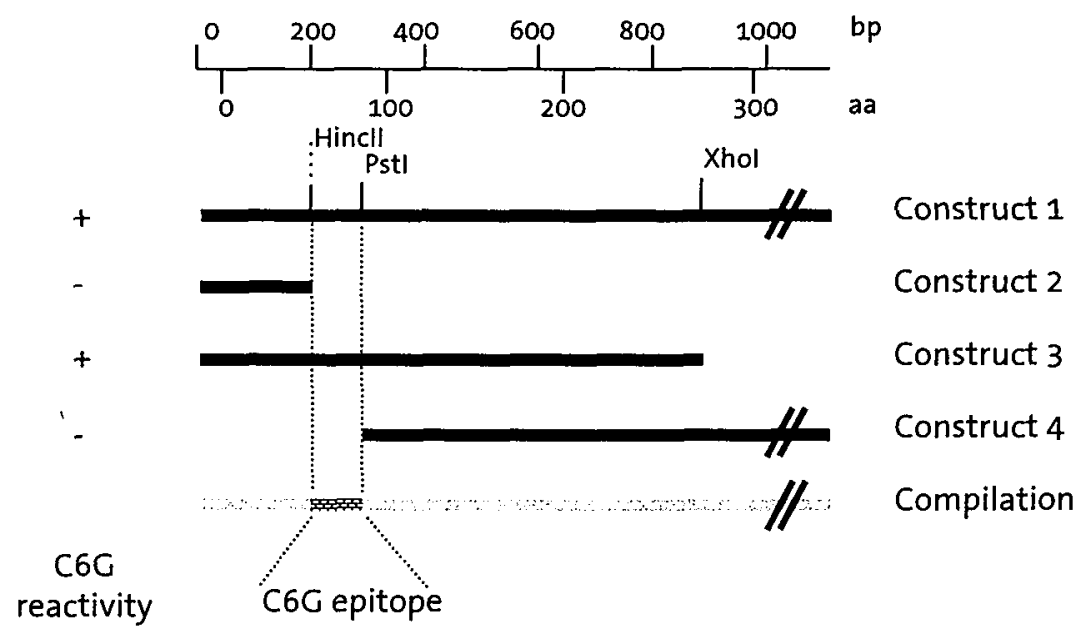

b

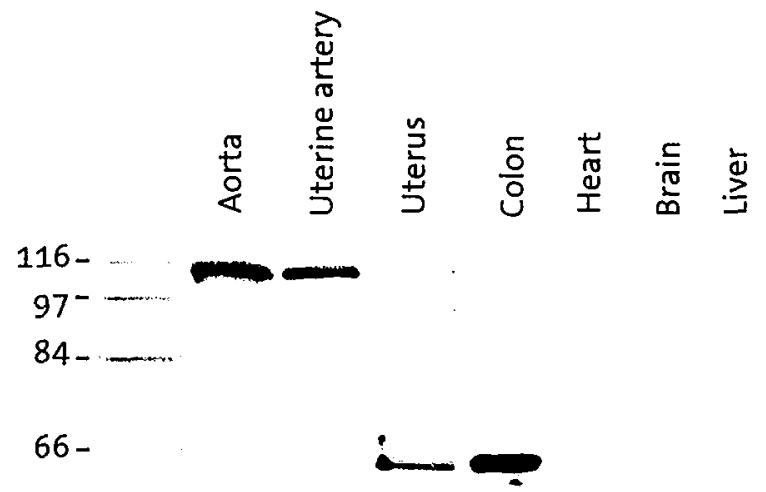

Figure 4.1 (a) Epitope mapping of the new antibody C6G. Deletion constructs of smoothelin-A cDNA were transfected in E. Coli M15, and expression of the protein fragments was induced. C6G reactivity of protein products is indicated. The epitope recognized by $C 6 G$ is located between amino acids 56 and 86 of the smoothelin-A protein (512-542 in smoothelin-B). (b) Western blots of smooth muscle tissues with antibody C6G directed against smoothelin. Vascular tissues show a prominent band at $110 \mathrm{kDa}$, smoothelin-B, whereas visceral smooth muscle displays the $59 \mathrm{kDa}$ smoothelin-A. Non-smooth muscle tissues do not express smoothelins.

with the $3000 \mathrm{nt}$ (but not the $1600 \mathrm{nt}$ ) mRNA was only seen after long exposure (figure 4.2). The smoothelin-B sequence contains a $\mathrm{CH}$ domain as does smoothelinA. The RT-PCR screen mentioned above revealed two alternative splicings in this $\mathrm{CH}$ domain. These alternative splicings were found in smoothelin-A as well as smoothelin-B mRNA. The three variants are defined by the presence or absence of exon 20 (figure 4.3). Smoothelin-B1 (without exon 20) and -B2 (with exon 20) are the major transcripts in vascular SMCs. The smoothelin-B1 CDNA has an open reading frame (ORF) of $2751 \mathrm{bp}$, coding for a 917 amino acid protein. Computer calculations indicate a molecular weight of $100 \mathrm{kDa}$, which corresponds to the 110 $\mathrm{kDa}$ protein found on Western blots. Tertiary structure analysis suggests that the 

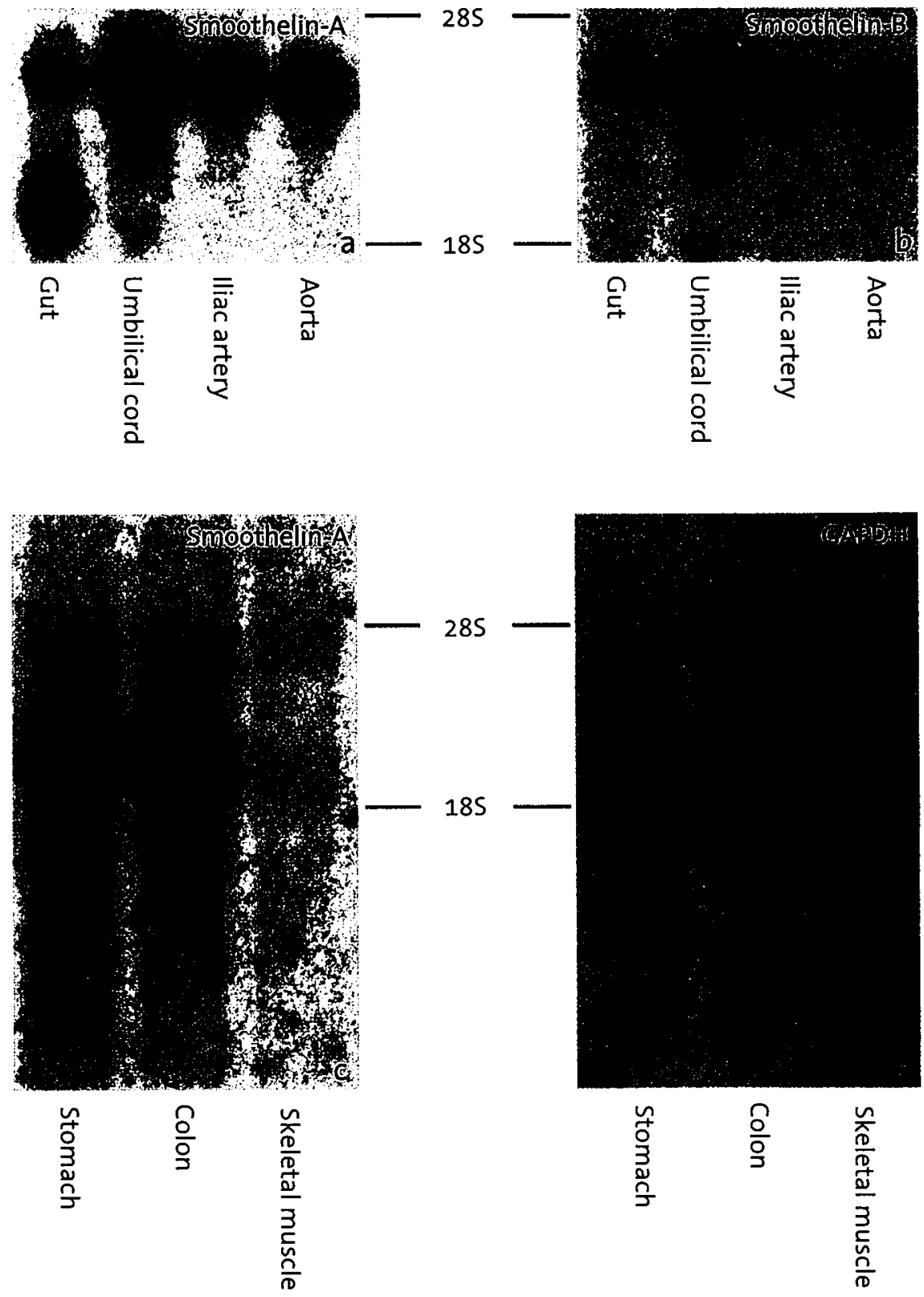

Figure 4.2 Northern blot of smooth muscle tissue and artery-derived smooth muscle primary cell culture. Panels (a) and (b) display a Northern blot of human gut and cultured SMCs derived from human umbilical cord artery, human iliac artery, and human aorta. Panel (a) shows these RNAs after hybridization with a probe containing a fragment of the smoothelin-A/B CDNA (exons 12-16 of the gene). Panel (b) (same blot) shows the result of hybridization with a probe containing a smoothelin-Bspecific fragment (exons 1-7). Panels (c) and (d) display a Northern blot of human stomach, colon, and skeletal muscle. The blot in panel (c) was hybridized with a smoothelin-A CDNA probe (long exposure), and the blot in panel (d) with a GAPDH CDNA probe. Notice the faint band for the vascular smoothelin-B transcript in the visceral samples, only visible after prolonged exposure. 
$N$-terminal 130 amino acids of the smoothelin- $B$ isoforms adopt an $\alpha$-helical configuration. Smoothelin-B2 cDNA has an ORF of $2745 \mathrm{bp}$, coding for 915 amino acids, producing a protein of $99 \mathrm{kDa}$. Smoothelin-B3 cDNA only contains the 5'70 bp of exon 20. The ORF of the A3/B3 variants continues through exon 21, causing the translational stop to be at the same position as in smoothelin-B1, with an insertion of 23 amino acids (figure 4.4).

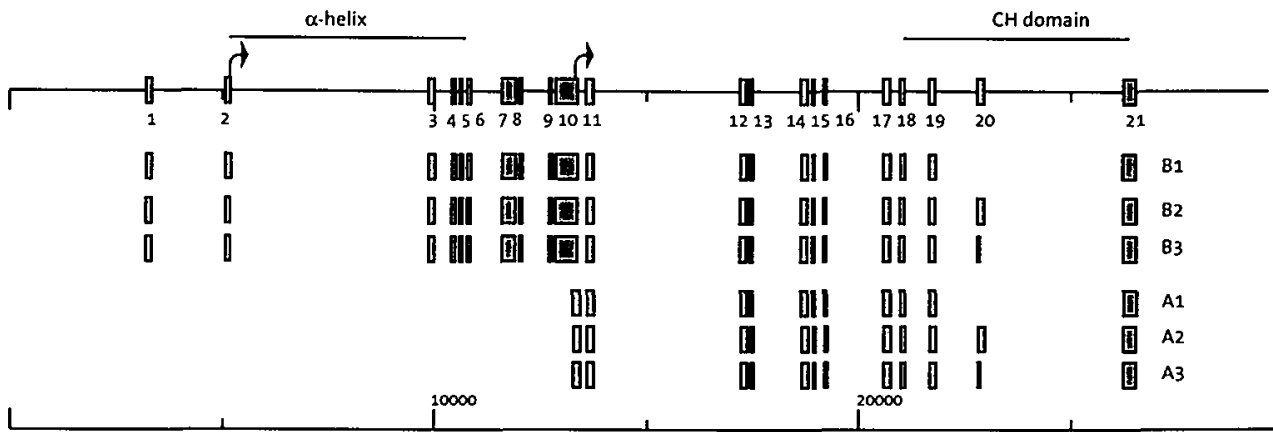

Figure 4.3 Structure of the human smoothelin gene. The bottom line shows the size (in bp) of the gene. The upper line displays the position of the 21 exons. The smoothelin transcripts (mRNAs) are represented by gray boxes. Exons coding for the putative $\alpha$-helical domain at the amino terminus of smoothelin-B, and exons coding for the $\mathrm{CH}$ domain are indicated. Translation initiation sites are depicted by arrows.

The homology of the $\mathrm{CH}$ domain to the actin-binding domain of members of the spectrin family is $45 \%$, irrespective of the splice variant. Alternative splicing of only the 5' part of exon 20 inserts an extra 23 amino acids homologous to the $\mathrm{CH}$ domain. Thus, the $C$-terminus of smoothelin-A3/B3 contains a part of this domain in duplicate. Expression of the different alternative splice variants of smoothelin-B and -A showed no coherent tissue specificity (figure 4.5), although prostate, lung, and oesophagus seemed to express less of the A1 variant compared with vascular tissues. All three splice variants were found in smooth muscle tissues investigated, with the exception of prostate, which did not express the A3/B3 variant. In most of the smooth muscle tissues tested, the expression level of the $B 3$ and $A 3$ variants was considerably lower compared with the other two variants. The human smoothelin-B1, $-B 2$, and $-B 3$ sequences have been deposited in the GenBank database under accession numbers Y13492, AY061971, and AY061972.

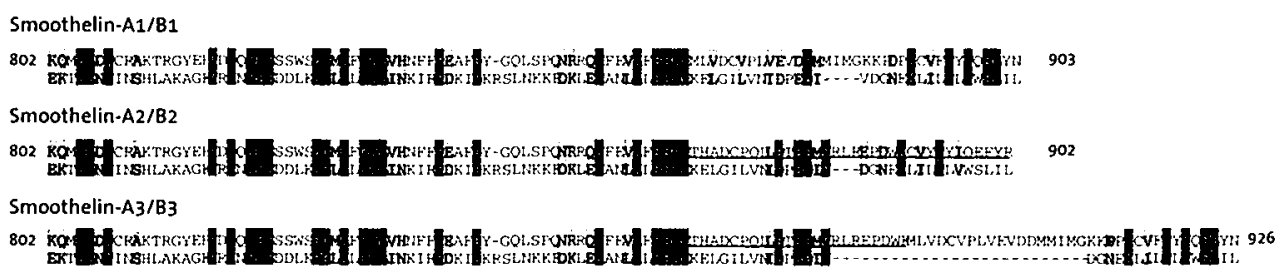

Figure $4.4 \mathrm{CH}$ domains of the three alternative splice variants of the smoothelin isoforms (top lines) are compared with the consensus actin-binding $\mathrm{CH}$ domain (bottom lines). Identical residues are shaded dark grey, similar residues are shaded light grey. Residues encoded by exon 20 are underlined. 
Cloning and analysis of the human smoothelin gene

A human cosmid library was screened with smoothelin-A CDNA probes, and two cosmids containing the full size smoothelin gene were selected. Twenty-one exons could be positioned by hybridization with cDNA fragments and sequencing (figure 4.3). As such, the present map of the gene represents a refining of the previously reported one ${ }^{491}$. The structure of the human smoothelin gene closely resembles the mouse smoothelin gene structure ${ }^{501}$. The first two exons are separated from the other exons by a $5 \mathrm{~kb}$ intron. Exon 10 is the largest exon, comprising $526 \mathrm{bp}$. In the middle of this exon, the transcription start site of smoothelin-A is located (figure 4.3). The exon-intron boundaries match the GT-AG splice recognition sequence. Between exons 16 and 17, a 50 bp TAAA repeat is found. The vascular specific part of the smoothelin-B CDNA sequence is coded for by the exons 1 through 9, and the $5^{\prime}$ half of exon 10 (figure 4.3). The $3^{\prime}$ half of exon 10 and the remaining ten exons are coding for both smoothelin isoforms. The sequences of the exons have been deposited in the GenBank database under accession numbers AF115551-AF115570. Screening of intronic sequences for internal exons with HEXON software revealed additional potential exons located in introns 11 and 19. These 'alternative' exons are conserved between mouse and human, contain correct splice sites, and their addition to the mRNA would not disturb the reading frame. However, these exons have not been found in any mature smoothelin mRNA so far.

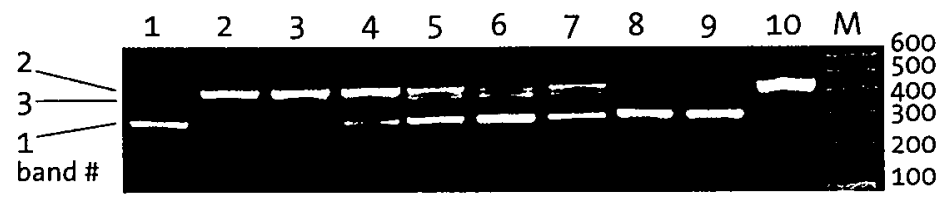

Figure 4.5 RT-PCR results on RNA extracted from various mouse tissues, showing the presence of three alternatively spliced smoothelin $\mathrm{CH}$ domains in all smooth muscle tissues, except prostate. Band 1 corresponds to smoothelin-A1/B1 isoforms, band 2 to smoothelin-A2/B2, and band 3 to smoothelinA3/B3, respectively. lane 1: uterus, lane 2: prostate, lane 3: lung, lane 4: esophagus, lane 5: stomach, lane 6: colon, lane 7: iliac artery, lane 8: carotid artery, lane 9: aorta, lane 10: heart, M: Marker (bp).

\section{Smoothelin transcription start site mapping and promoter reporter assays}

Primer extension of RNA extracted from human ileum with a smoothelin specific primer produced a fragment of $83 \mathrm{nt}$ (figure 4.6a). Together with the 5'RACE results, which reveal that in vascular tissues a much larger transcript is generated, this shows that the smoothelin-A and -B CDNA have completely different 5 ' ends. These $5^{\prime}$ ends are separated by approximately $10 \mathrm{~kb}$ of genomic sequence, indicating that two different transcription start sites may be used. Sequences directly upstream of the putative transcription initiation sites have been screened for promoter/enhancer elements (figure $4.6 \mathrm{~b} / \mathrm{c}$ ). The putative promoter regions of neither smoothelin-A nor smoothelin-B contain a TATA-box, but a number of promoter/enhancer elements have been identified. Among them are $\mathrm{CArG}$ (like) boxes, E-boxes, AP- 2 and SP-1 sites, a TGF- $\beta$-control element, and GATA sites. 
a

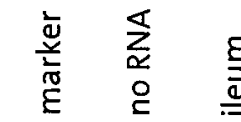

118

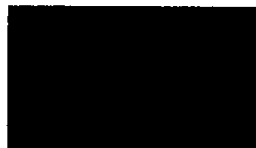

100

82

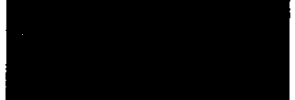

66

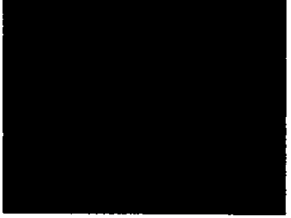

Figure 4.6 Primer extension results and cis-acting elements present in the smoothelin-A or smoothelin-B promoter region. (a) Primer extension of RNA extracted from human ileum using a smoothelin specific primer (underlined in (b)) produced a product of $83 \mathrm{nt}$, showing that transcription starts at the site indicated in (b). (b) Smoothelin-A promoter region. Cis-acting elements are shaded. The transcription initiation site of smoothelin-A is depicted by an arrow. Exon sequences are bold. The sequence of the primer used for primer extension analysis is underlined. (c) Smoothelin-B promoter region with indicated cis-acting elements. Exon sequences are bold.

b

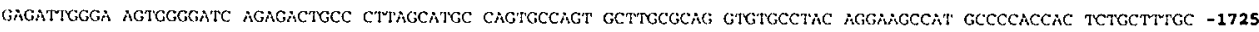

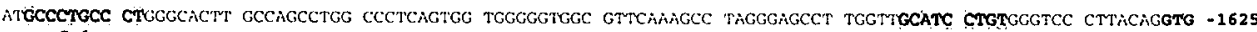
Sp1

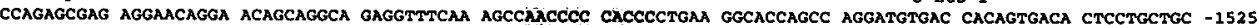

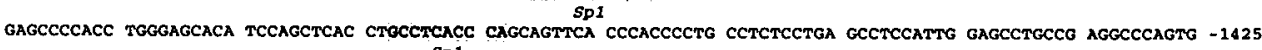

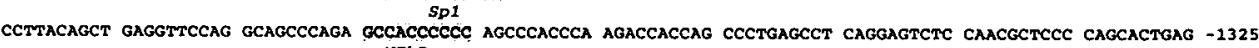

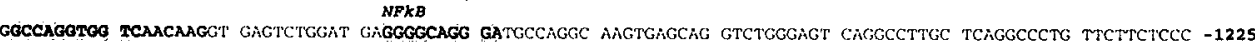
MYOD

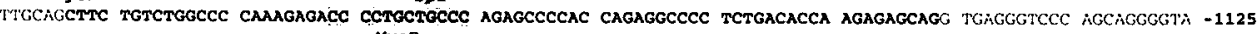

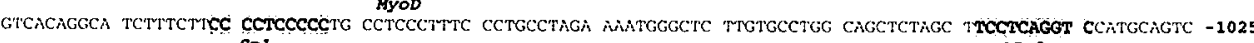
CCFGATACTG CACCCCACCC CTGAAGTGTC CCCGCCCCCA GCTAGGCCAG COTCCCCCTC CACCCCCATG GCTAGRGCCC TCCCCTGCAG CCTTOAAGG -92 Spl C-ETS-1 Spl Spl Spl SARG-11Ko

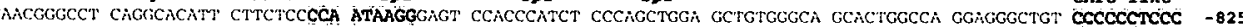

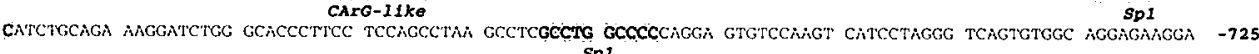

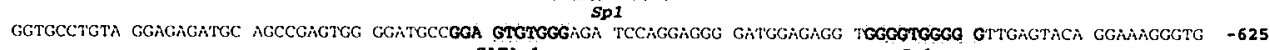
GATA-1

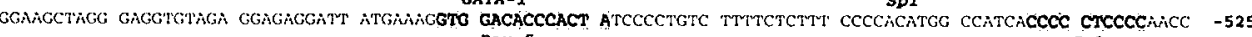
TGCCAGACGT GGCTGGACCC CGACCCTGCC AACGCTCCCT GTCGGTGCXC AGCCCCCGCC AACCAGCCCA GAACCGAGGT ACTACCTATT CTCACCCTCC -425 Sp1 ce-Btg-1 STCGTGCAC AGCCCCCGCC AACCAGCCCA GAACCGAGGT MCTACCTATT CTCACCCTCC - 25

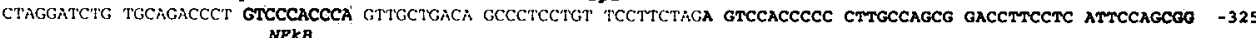
NPKB

gCTEGCTCTG TGCGgGATCG TGTCCACAAG TTCACATCTG ATTCTCCTAT GGCTGCTAGG CTCCAGGATg GCACACCCCA GGCTGCCCTA AGTCCCCTGA -225 $S p 1$ CCCCCGCAAG GCTCCTGGGC CCCTCCCTCA CCAGCACCAC CCCTGCCTCC TCCTCCAGCG GCTCCTCCTC TCGGGGCCCC AGTGATACCT CCTCCCGGTT -125

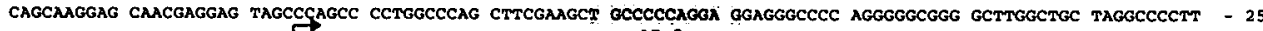

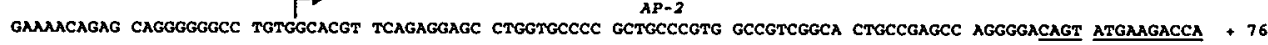
CATTCAC

\section{C}

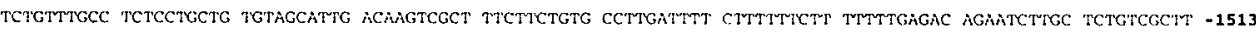

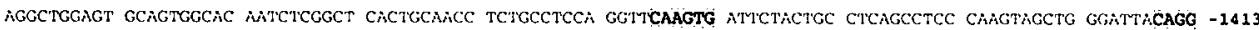

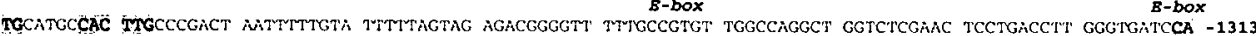

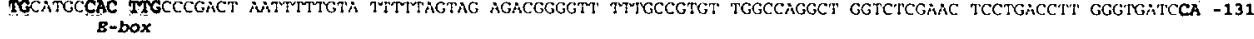

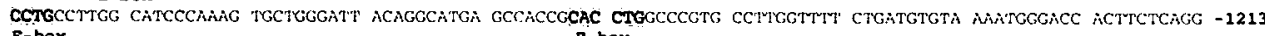
B-box GGTGTGGGGA TGAATGMA TGACACATGG ANAGACTCT CAGTGTGGA ACTGGGCCTT TOCTTAGATG GGGGCTGGGG GGTGTCACAG AGGACCIGA -1113

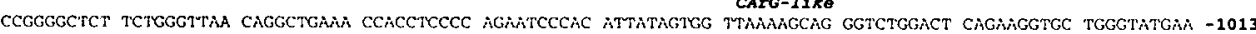

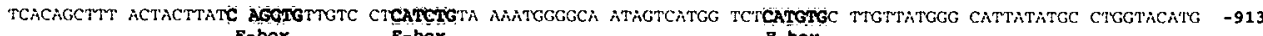
$\begin{array}{lll}E-b o x & E-b o x & E-b a x\end{array}$ 


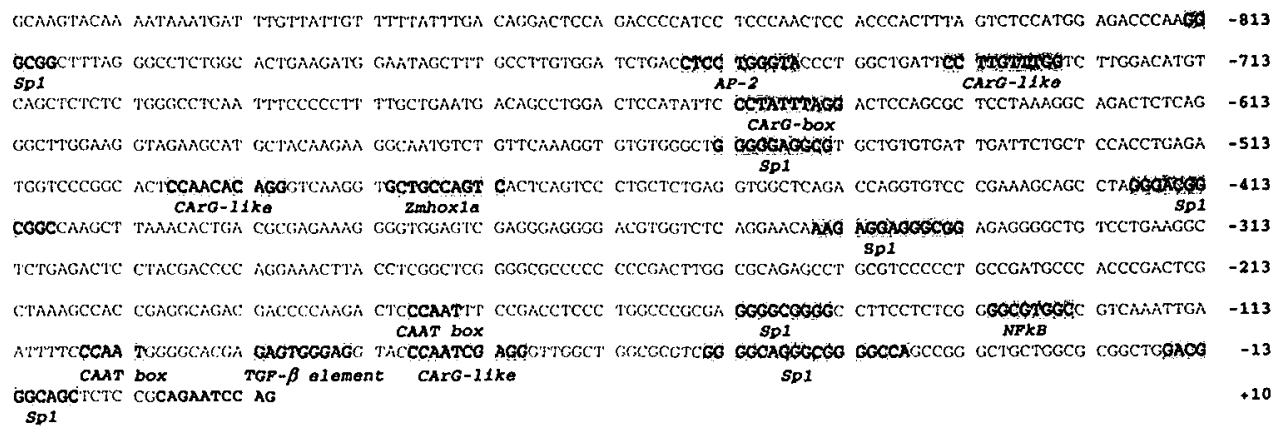

Comparison between human and mouse putative promoter sequences of smoothelin-B revealed a high degree (66\%) of homology for about $700 \mathrm{bp}$ upstream of the transcription initiation site. The putative smoothelin-A promoter, which also contains exons of the smoothelin-B-specific part, showed an even higher degree of conservation (73\%). Intronic sequences were generally as conserved as exon sequences ${ }^{501}$.

Promoter reporter assays indicated that the region upstream of the first smoothelin-B exon has promoter activity (figure 4.7a). A minimal promoter construct containing 84 bp of the region upstream of the transcription start site was able to drive luciferase expression, albeit at low levels. Increasing the size of the promoter fragments up to $750 \mathrm{bp}$ resulted in higher expression. Larger promoter fragments of 1097 and 1616 bp, in contrast, gave a lower luciferase activity. The highest activity was conferred by a construct containing 3372 bp of the promoter. Apart from the minimal promoter, all constructs that were tested showed considerable specificity for SMCs.

A smoothelin-A promoter fragment of $370 \mathrm{bp}$ was not able to drive luciferase expression in any of the cell types tested. The minimal promoter for the smoothelin-A transcript therefore has to include elements upstream of this region. A larger smoothelin-A promoter fragment produced variable results in the vascular PAC1 SMCs that were used for the promoter assays. However, in the non-SMC cell lines tested, this $1613 \mathrm{bp}$ fragment displayed no promoter activity at all (figure 4.7a). Because of the variation, the same fragment was cloned upstream of another reporter gene, $\beta$-galactosidase. In this assay, numerous positive cells were identified, confirming that this region of the smoothelin gene exerts promoter activity (figure $4.7 \mathrm{~b}$ ). 
a
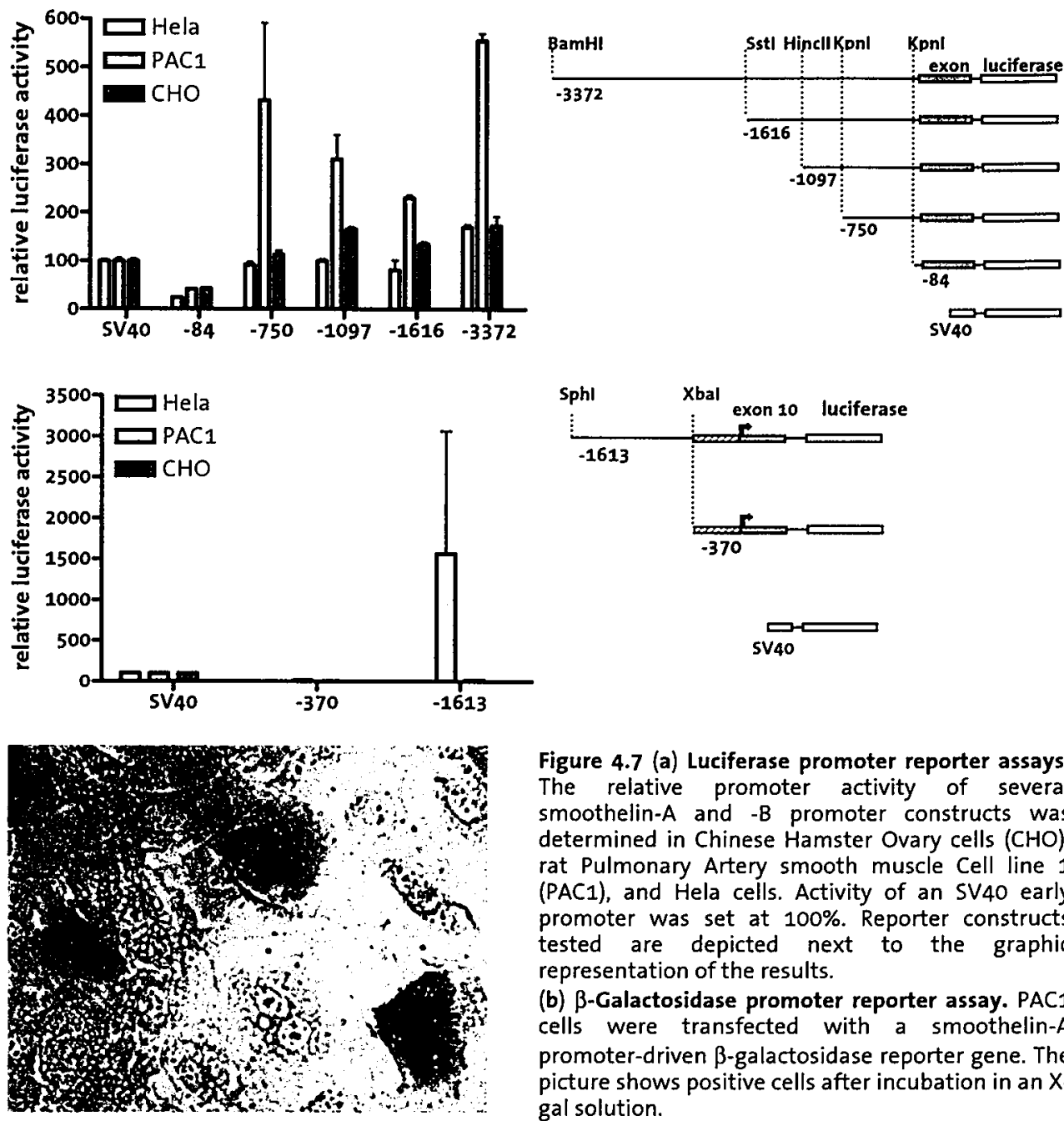

Figure 4.7 (a) Luciferase promoter reporter assays. The relative promoter activity of several smoothelin-A and $-B$ promoter constructs was determined in Chinese Hamster Ovary cells (CHO), rat Pulmonary Artery smooth muscle Cell line 1 (PAC1), and Hela cells. Activity of an $5 V 40$ early promoter was set at $100 \%$. Reporter constructs tested are depicted next to the graphic representation of the results.

(b) B-Galactosidase promoter reporter assay. PAC1 cells were transfected with a smoothelin-A promoter-driven $\beta$-galactosidase reporter gene. The picture shows positive cells after incubation in an $X$ gal solution.

\section{Discussion}

The contractile apparatus of SMCs is constructed around SMC-specific isoforms of actin and myosin proteins. Smooth muscle specific proteins such as SM-calponin, $S M 22 \alpha$, and h-caldesmon are associated with $\alpha$-SMA or myosin ${ }^{6,20,361,502}$. The function of these proteins is still unclear. Smoothelins are a recent addition to the group of actin-associated SMC-specific proteins. They share a number of characteristics with SM-calponin and SM22 $\alpha$, such as the presence of a $\mathrm{CH}$ domain, and transient expression in the primitive heart during embryogenesis ${ }^{82}$. However, smoothelin expression is restricted to contractile SMCs, whereas SM-calponin and 
SM22 $\alpha$ are also expressed in less differentiated SMCs. As such, smoothelin has been used as a marker for the differentiated contractile phenotype of $S M C s^{22,83}$. Previously, we have characterized smoothelin as a structural protein, specific for contractile smooth muscle tissues. A smoothelin CDNA cloned from visceral smooth muscle tissue was demonstrated to code for the $59 \mathrm{kDa}$ smoothelin- $\mathrm{A}^{23}$. However, Western blotting of smooth muscle tissues in several species revealed that two isoforms of smoothelin exist ${ }^{80,434}$. In addition to the $59 \mathrm{kDa}$ smoothelin-A in visceral tissues, we found an immunoreactive product of $110 \mathrm{kDa}$ in vascular tissues. Analysis of a variety of smooth muscle tissues indicated that this protein, designated smoothelin-B, is specific for contractile vascular SMCs in adults ${ }^{80,83}$. Here, we present evidence that in humans, like in mice ${ }^{81}$, this smoothelin-B isoform is translated from a $3000 \mathrm{nt}$ mRNA, whereas smoothelin-A is translated from a $1600 \mathrm{nt}$ mRNA. The molecular weight of smoothelin-B as estimated from the cDNA open reading frame is $100 \mathrm{kDa}$, which is in good agreement with the approximately $110 \mathrm{kDa} M W$ observed in Western blotting analyses (small inter-species differences have been found, for instance the pig smoothelin-B is $115 \mathrm{kDa})^{83}$. Computer assisted structural analysis of the putative amino acid sequence revealed potential $\alpha$-helical structures. The most prominent one is a 130 amino acid domain at the $\mathrm{N}$ terminus of smoothelin-B. Such a domain may be involved in the formation of filamentous structures, or may have a function in coiled-coil interactions with other molecules, as described for many cytoskeletal and cytoskeleton-associated proteins ${ }^{503}$. At the $\mathrm{C}$-terminus, a $\mathrm{CH}$ domain with homology to the actin-binding domain of the spectrin family is present in smoothelin-B (as in smoothelin-A). Both smoothelins contain only one $\mathrm{CH}$ domain, which binds directly to actin ${ }^{504}$. In contrast, similar single $\mathrm{CH}$ domains found in other actin-associated proteins such as SM22 $\alpha$ and SM-calponin appear not to bind actin directly ${ }^{505}$. Alternative splicing of exon 20 can generate three different $\mathrm{CH}$ domains in smoothelin-A and - $\mathrm{B}$. Two of these alternative splicings in smoothelin- $B$ have been described previously ${ }^{490}$. However, we show that a third alternative splicing occurs in all smooth muscle tissues except prostate, and that these alternative splicings occur in both smoothelin-A and smoothelin-B mRNA. The lack of a tissue specific expression of any of these variants implies that these alternatively spliced actin-binding sites may have little physiological significance. Apparently, neither the $\mathrm{N}$-terminal $\alpha$ helical structure nor the alternative splicing products of the C-terminus of both smoothelins influence the subcellular distribution of smoothelin and more particular its colocalization with actin. Therefore, our present data indicate that the smoothelin isoforms and their splice-variants perform, at least in part, similar functions in visceral and vascular SMCs. The influence of the alternative splicings on the affinity of the different smoothelin isoforms for actin is currently under investigation.

In situ hybridization and radiation hybrid analysis has shown that smoothelins are encoded by a single copy gene ${ }^{433}$. Consequently, three options were at hand to explain the occurrence of the smoothelin-B isoform in vascular smooth muscle 
tissue: (1) extensive posttranslational modification of smoothelin-A; (2) alternative splicing; (3) alternative promoters.

If posttranslational modification were responsible for the increase in molecular weight, the two isoforms of smoothelin would be translational products of the same mRNA. However, Northern blotting revealed two mRNAs both hybridizing to the smoothelin-A CDNA. A 1600 nt transcript was found in visceral tissues, whereas a $3000 \mathrm{nt}$ mRNA was found in vascular SMCs. The sizes of these two mRNAs correspond with the two protein isoforms of smoothelin. The weak $3000 \mathrm{nt}$ band observed in visceral tissues is most likely due to the presence of blood vessels, although different expression ratios of the two major isoforms in various smooth muscle tissues cannot be completely ruled out. Thus, posttranslational modification cannot be responsible for the increase in $\mathrm{MW}$ of the vascular isoform. Alternative splicing was investigated by RT-PCR with primers enclosing the splice sites in the smoothelin-A mRNA. This revealed the presence of an additional exon (20), or a part of this exon in the mature vascular mRNA of both smoothelin-A and -B. Addition of this exon modifies the C-terminus of the proteins, making them 2 amino acids shorter. In chicken embryonic striated muscle cells and in some prostate tumor cell lines, corresponding alternatively spliced mRNAs have been found ${ }^{82,506}$. The minor decrease of the ORF caused by the alternative splicing of exon 20 dismisses this mechanism as the origin of smoothelins-A and -B. In conclusion, alternative splicing does not generate the smoothelin-A and -B isoforms, but provides diversity in the smoothelin $\mathrm{CH}$ domain, which is encoded by exons 18-21.

Consequently, alternative promoters are the remaining option for the generation of the smoothelin-A and $-B$ isoforms. Although it is not common, a number of these promoter systems have been described ${ }^{507}$. Primer extension and $5^{\prime}$ RACE experiments provided evidence for two independent transcription start sites. The sequences located $5^{\prime}$ of both transcription start sites contain a number of elements known to be involved in gene expression regulation in SMCs, such as conserved CArG elements and E-boxes. Because the transcription start site of smoothelin-A is located inside exon 10, promoter elements may be present in upstream exons. Transfections of promoter-reporter constructs showed that regions upstream of the transcription initiation sites of smoothelin-B as well as smoothelin-A exert promoter activity. Moreover, both smoothelin promoters were able to drive expression in a SMC-specific way, although activity of the 1613 bp smoothelin-A promoter fragment was variable in PAC1 cells. The tissue specificity of the smoothelin-A promoter and its observed activity in two reporter systems nevertheless convincingly demonstrate the ability of this sequence to drive smoothelin-A expression in SMC. The lack of activity of the $370 \mathrm{bp}$ putative smoothelin-A promoter region may be explained by the fact that this region consists entirely of exon sequence. The larger 1613 bp smoothelin-A promoter construct contains, among other elements, two conserved CArG boxes close to each other, present in a region that has a high homology between mouse and 
human, even though it is in an intron. It is tempting to speculate that these elements may play a role in the regulation of smoothelin-A expression, like they do in other smooth muscle restricted genes.

The sequence directly upstream of the smoothelin-B transcription start site clearly displays promoter activity as can be deduced from comparison with the SV4O positive control. The lower luciferase activity of the constructs containing 1097 and $1616 \mathrm{bp}$ of the smoothelin-B promoter compared with the construct containing $750 \mathrm{bp}$ indicates that two or more repressor elements may be present in the region between -750 and $-1616 \mathrm{bp}$. Interestingly, this reduction of promoter activity only occurs in SMCs. Strong SMC-specific enhancers appear to be present between -1616 and -3372 bp of the smoothelin-B promoter, as evidenced by a large increase in promoter activity of the 3372 bp smoothelin-B promoter construct in SMCs. Numerous cis-acting elements can be identified in this region. The functionality of these elements however remains to be determined by footprinting, gel mobility shift assays, or mutational analysis.

The use of alternative promoters sets the smoothelins apart from the other described SMC-specific genes. It apparently enables SMCs to regulate smoothelin expression in a strict tissue specific fashion. Physically separated promoters provide a way to respond differentially to extracellular signals (e.g. hormones). Comparison of the two promoters may reveal important insight into gene regulation in different SMC populations.

\section{Acknowledgements}

The authors would like to thank Mark Oldheten and Edward Verkaar for their contribution in the sequence analysis, Wiel Debie and Berry Souten for technical assistance, and Prof. F.C.S. Ramaekers for providing the opportunity to carry out this study. 



\title{
Role of serum response factor and myocardin in transcriptional regulation of smoothelins
}

\author{
Sander Rensen*, Petra Niessen*, Pieter Doevendans**, \\ Joseph Miano***, Guillaume van Eys* \\ - Department of Genetics and Cell Biology, Cardiovascular Research Institute Maastricht, \\ Universiteit Maastricht, Maastricht, The Netherlands \\ "* Department of Cardiology, Heart Lung Center Utrecht, Utrecht, The Netherlands I \\ Interuniversity Cardiology Institute, Utrecht, The Netherlands \\ *** Department of Medicine, University of Rochester Medical Center, Rochester, NY, USA
}




\section{Abstract}

Smoothelins (SMTNs) are specifically expressed in contractile smooth muscle cells (SMCs). The two major isoforms, SMTN-A and -B, are thought to arise from alternative promoters. In this study, we have definitely established the existence of the intragenic promoter regulating transcription of SMTN-A in cultured PAC1 $S M C s$, and show that its activity is in part dependent on two evolutionarily conserved CArG elements that bind the serum response factor (SRF). SMTN-A promoter activity was consistently lower than SMTN-B- and $\alpha$-smooth muscle actin promoter activity in PAC1. Mutation of the proximal CArG box (CArG-near) of SMTN-A attenuated promoter activity by $15 \%$, whereas mutation of the distal CArG box (CArG-far) did not affect promoter activity significantly; mutating both CArG boxes reduced activity by $32 \%$. Electrophoretic mobility shift assays revealed that CArG-far interacted weakly with SRF. CArG-near-SRF interaction was much stronger, but considerably weaker than seen with the intronic CArG element within smooth muscle-calponin. Myocardin, a potent transcriptional co-activator of SRF that acts through CArG boxes, augmented SMTN-A promoter activity by 2.5 -fold in a CArG-near-dependent manner. Myocardin had little effect on the SMTN-B promoter. These results reveal fundamental differences in the regulation of SMTN-A and $-B$ in vascular smooth muscle cells, and establish a role for SRF-myocardin in the regulation of SMTN-A.

\section{Introduction}

Smooth muscle cells (SMCs) are of key importance for the correct functioning of organs involved in processes as diverse as blood pressure homeostasis, breathing, and intestinal peristalsis. To this end, these cells are equipped with a unique set of proteins allowing contraction and relaxation. Many of these proteins are exclusively or predominantly expressed in SMCs, including $\alpha$-smooth muscle actin $(\alpha-S M A)$, smooth muscle myosin heavy chain isoforms (SM-MHC), SM22 $\alpha$, smooth muscle calponin (SM-calponin), and smoothelins (SMTN-A \& $-B)^{1,508}$. During physiological remodeling and after injury of smooth muscle tissues, SMCs fulfil a distinct role aimed at restructuring or repairing, thereby securing structural integrity. To accomplish this, they express a different set of proteins that allow proliferation, extracellular matrix (ECM) remodeling, and migration. However, high-level expression of contractile proteins is not compatible with expression of proteins involved in the repair function. Therefore, SMCs with different phenotypes can be identified: 1) contractile SMCs, which express contractile proteins at high levels, and 2) synthetic SMCs, which upregulate ECM-degrading enzymes and downregulate contractile gene expression. SMCs can shuttle between these phenotypes, a process called phenotypic modulation ${ }^{4}$. During modulation towards the synthetic phenotype, expression of different contractile proteins does not 
diminish uniformly. Synthetic SMCs still express considerable amounts of $\alpha-5 M A$, SM22 $\alpha$, and SM-calponin. Expression of SM-MHC and SMTNs, however, is rapidly lost in modulating SMCs ${ }^{23,55,57,83,509}$. Therefore, SM-MHC and SMTNs are widely used markers of the contractile SMC phenotype.

In recent years, many studies have been performed to elucidate the mechanisms by which SMCs regulate the expression of proteins that are associated with a specific phenotype. For this purpose, promoters of genes expressed primarily in contractile SMCs have been investigated. It has become clear that many cis-elements contribute to promoter activity of these genes, but one specific element, the CArG box, has been shown to be of critical importance for their expression ${ }^{258,261,510}$. This element, with the sequence $C C\left(A / T_{6}\right) G G$, is recognized by serum response factor (SRF), a transcription factor of the MCM1-Agamous-Deficiens-Serum response factor (MADS) box family ${ }^{268}$. Because SRF is widely expressed and not regulated in a SMC phenotype-specific way, it has long remained unclear how this factor would be able to direct expression of genes specifically in contractile SMCs. Recently, myocardin has been identified as an SRF cofactor with a cardiac/smooth muscle restricted expression pattern ${ }^{168}$. It is a member of the SAP (SAF-A/B, Acinus, PIAS) family of transcriptional regulators which was shown to strongly enhance transcription of several SMC-specific genes ${ }^{39,114,242,279}$. Myocardin levels declined upon modulation towards a synthetic phenotype ${ }^{39}$. Hence, this transcriptional cofactor may contribute to regulation of the expression of genes that are specifically associated with the contractile SMC phenotype.

Although the expression of SMTNs is strongly correlated with the contractile phenotype, little is known about their transcriptional regulation. Expression of the SMTN-A and -B isoforms is thought to be mediated by two promoters separated by $10 \mathrm{~kb}$ in the human SMTN gene ${ }^{511}$. The promoter upstream of exon 1 directs expression of SMTN-B mRNA in vascular SMCs, whereas an intragenic promoter located upstream of exon 10 regulates SMTN-A expression in both vascular and visceral SMCs. Like in other SMC marker gene promoters, conserved CArG boxes are found in the SMTN promoter regions ${ }^{501}$.

The first aim of the current study was to confirm the existence of the intragenic promoter in a well-defined in vitro model of SMCs. The second aim was to determine the functional importance of the two CArG boxes that are present in this region. Finally, the ability of myocardin to activate the SMTN-A and SMTN-B promoters was assessed. The results reveal that intragenic SMTN-A promoter activity is partly dependent on CArG boxes, although their role is not as dominant as in other SMC-specific genes associated with the contractile phenotype. SMTN-B promoter activity is higher than SMTN-A promoter activity, but not augmented by myocardin. Collectively, these findings suggest that distinct circuits of transcriptional control exist for SMTN-A and SMTN-B expression, and that nonCArG-dependent pathways may predominate in the control of SMTN expression. 


\section{Materials and methods}

\section{Cell culture}

Pulmonary artery cells of the rat (PAC1) $)^{245,246}$ were cultured in Dulbecco's Modified Essential Medium supplemented with $10 \%$ fetal calf serum, $50 \mu \mathrm{g} / \mathrm{ml}$ gentamicin and $200 \mu \mathrm{M}$ L-glutamine (Life technologies, Paisley, UK).

\section{Construction of reporter plasmids}

A SMTN-A promoter fragment was obtained by PCR amplification of a cosmid containing the entire mouse smoothelin gene ${ }^{501}$, using the following primers: forward 5'GCCTTCCTTATCGAGGTGTC3', reverse 5'CCCAAGCTTCTGGCTCCCCAGTGC CAAC3', and Pfu Taq DNA polymerase (Stratagene, La Jolla, CA). PCR parameters were $94^{\circ} \mathrm{C}$ for $5 \mathrm{~min}$, followed by $35 \mathrm{cycles}$ of $94^{\circ} \mathrm{C}$ for $30 \mathrm{sec}, 58^{\circ} \mathrm{C}$ for $30 \mathrm{sec}, 72^{\circ} \mathrm{C}$ for $1 \mathrm{~min}$, and a final step of $72^{\circ} \mathrm{C}$ for $5 \mathrm{~min}$. PCR products were cloned into PGEM-T Easy (Promega, Madison, WI) and their sequence was verified. Promoter fragments were subcloned into the luciferase reporter vector PGL3-basic using restriction enzymes Xhol-HindIII, Kpnl-HindIII, and Smal-HindIII, as indicated in figure 5.1. In this way, promoter fragments $M-1560, M-1315, M-851, M-658$, and $M-208$ were generated with numbers corresponding to the size of the promoter fragment (see figure 5.1). All fragments extend to +58 bp relative to the SMTN-A transcription start

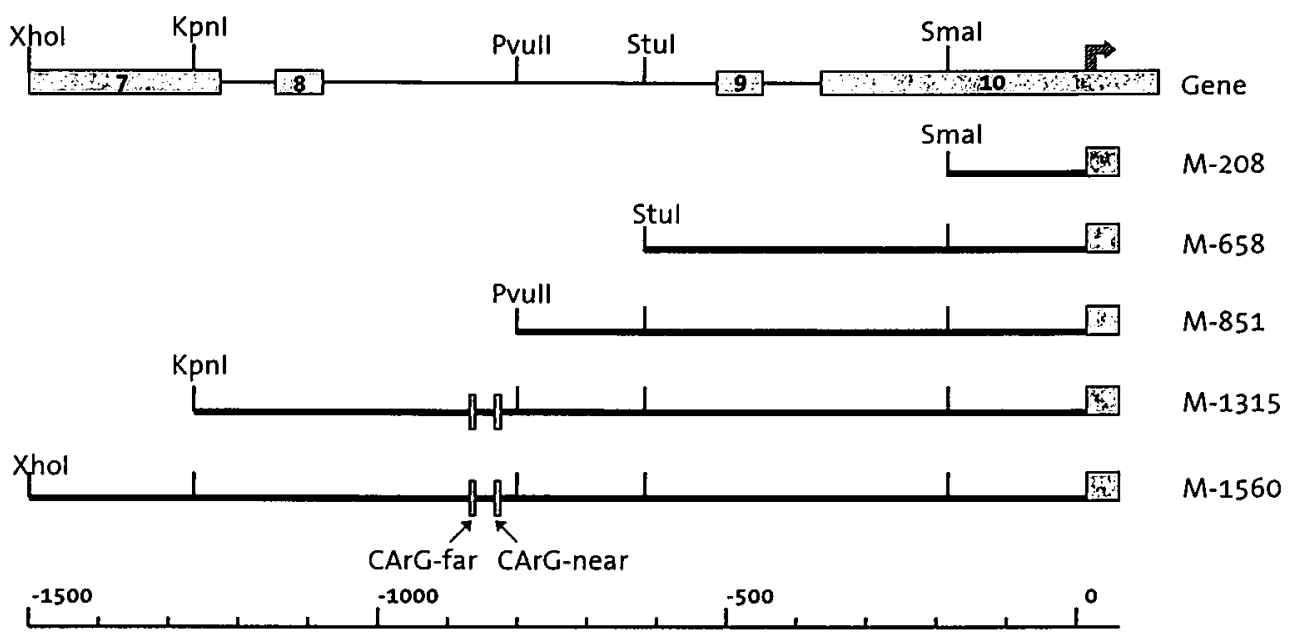

Figure 5.1 Schematic representation of mouse SMTN-A promoter deletion constructs. The upper line represents the SMTN gene structure, exons are indicated by grey boxes and numbered. Restriction sites used for cloning purposes are shown. The arrow indicates the transcription start site of SMTN-A. The lower line shows the scale in bp.

site $^{501}$. Construction of the $\alpha$-SMA (-195 bp) and SMTN-B promoter reporter plasmids (-1616 bp) has been described before ${ }^{246,511}$.

To determine whether the two CArG boxes of the human SMTN-A promoter 
contribute to its transcriptional activity, they were mutated in the context of the reporter plasmid previously described to be active in $P A C 1^{511}$. This $\mathrm{H}-1613$ plasmid was subjected to site-directed mutagenesis employing a high fidelity Pfu DNA polymerase (Stratagene). The proximal $C C(A / T)$ sequence of each $C A r G$ box was mutated to GTC (see figure 5.2), which is known to abrogate binding of SRF ${ }^{510}$. The resulting plasmids are referred to as CArGFmut, CArGNmut and CArGNFmut to indicate mutation of either the distal CArG-far box (Fmut), the proximal CArG-near box (Nmut), or mutation of both (NFmut). The mutated plasmids were verified by sequencing.

CAGCCTTGAAAGGCAACGGGCCTCAGGCACATTCTTCTCCCCAATAAGGGAGT Wild type

WT CAGgtCTGAAAGGCAACGGGCCTCAGGCACATTCTTCTCCCCAATAAGGGAGT CArC far mutant CAGCCTTGAAAGGCAACGGGCCTCAGGCACATTCTTCTCCgtCATAAGGGAGT CArG near mutant CArGFmut CAggtctgaAaggCAACGgGCTCAGGCACATTCTTCTCCgtcataAggGagT Double mutant

Figure 5.2 Sequence of CArG mutations introduced into the human SMTN-A promoter reporter construct $H-1613$. CArG boxes are shown in bold, mutations are underlined and in small case.

\section{Transient transfections and luciferase assays}

$1.5 \times 10^{5}$ PAC1 cells per well were seeded in 12-well plates and allowed to attach overnight. The next day, $2 \mu \mathrm{g}$ of reporter plasmid were transfected into the $60-70 \%$ confluent cells using Fugene- 6 transfection reagent (Roche Diagnostics, Brusseis, Belgium) according to the provided instructions. A renilla reporter gene was included to normalize luciferase activity. To test the effects of myocardin expression on SMTN-A promoter activity, co-transfection of a pCDNA3.1 vector carrying myocardin $\mathrm{CDNA}^{39,510}$ was performed. In these experiments, control cells were transfected with equivalent amounts of backbone vector without insert. After 48 hours, analysis of luciferase expression in cell lysates was performed using a dual luciferase assay system (Promega) in a Biocounter M1500 luminometer (Lumac, Landgraaf, The Netherlands) or an AutoLumat LB 953 luminometer (EG\&G Berthold, Gaithersburg, MD). All transfections were done in quadruplicate and repeated in one or more independent experiments. Data are reported as mean \pm standard error of the mean and analyzed by one-way ANOVA, followed by Bonferroni's multiple comparison test. Values of $P<0.05$ were considered statistically significant.

\section{Nuclear extract preparation and electrophoretic mobility shift assays (EMSAs)}

PAC1 nuclear extracts were prepared from cells grown to confluency in $100 \mathrm{~mm}$ dishes using a Nuclear Extract kit (ActiveMotif, Rixensart, Belgium) and stored at $-80^{\circ} \mathrm{C}$. Protein concentrations were measured by the BCA assay (Pierce, Rockford, IL). Probes for EMSA were obtained by end-labeling HPLC-purified oligonucleotides corresponding to the CArG boxes of the human SMTN-A promoter (Sigma-Genosys, The Woodlands, TX). The oligonucleotides used are indicated in figure 5.3 (dashed lines), and extend from -881 to -911 (CArG-near) and -919 to -947 (CArG-far). In addition, the intronic CArG box $\left(I C_{1}\right)$ of the $S M$-calponin gene, which has previously 
been shown to strongly bind $\mathrm{SRF}^{510}$, was used to compare SRF-binding affinities. 3.5 pmol of sense and antisense oligos were separately labeled using $10 \mu \mathrm{Ci}$ of $\left[\gamma^{32} \mathrm{P}\right]$ ATP (at $4000 \mathrm{Ci} / \mathrm{mmol}$ ) and T4 polynucleotide kinase (Promega). The labeled probes were mixed and annealed by slowly cooling to room temperature after heating to $70^{\circ} \mathrm{C}$. Unincorporated nucleotides and single-stranded oligos were removed by Sepharose G-50 spin columns (Amersham Biosciences, Piscataway, NJ). The labeling efficiency of the smoothelin probes was comparable, whereas labeling of the SMcalponin probe was 3-fold less. For competition experiments, unlabeled wild-type oligonucleotides or oligonucleotides mutated in the same way as the CArGFmut or CArGNmut reporter constructs (see figure 5.2) were used at 20-fold excess.

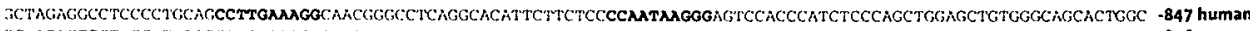

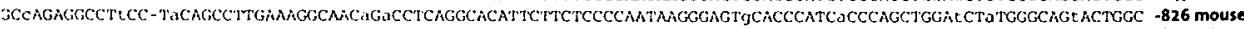

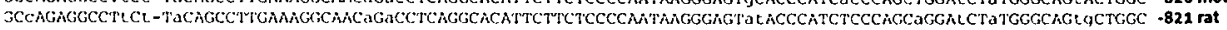

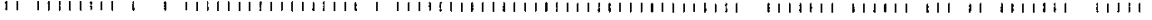

Figure 5.3 Sequence comparison of CArG box-containing region in the SMTN-A promoter. Nonconserved nucleotides are indicated by small case letters. The CArG boxes are shown in bold. Dashed lines indicate probes used for EMSA experiments. Dashes below the sequence indicate conserved nucleotides.

Reaction mixtures for EMSA containing $5 \mu \mathrm{g}$ of nuclear extract and $4 \%$ glycerol, 1 $\mathrm{mM} \mathrm{MgCl}, 0.5 \mathrm{mM}$ EDTA, $0.5 \mathrm{mM} \mathrm{DTT}, 50 \mathrm{mM} \mathrm{NaCl}, 10 \mathrm{mM}$ Tris- $\mathrm{HCl}(\mathrm{pH} 7.5)$, and $0.05 \mathrm{mg} / \mathrm{ml}$ poly(dl-dC) were incubated for $10 \mathrm{~min}$ on ice. Subsequently, approximately $45000 \mathrm{cpm}$ of labeled probe $\left(15000 \mathrm{cpm}\right.$ for $\left.\mathrm{IC}_{1}\right)$ was added and incubated for an additional $20 \mathrm{~min}$ at room temperature. For supershifts, $1 \mu \mathrm{l}$ of SRF antibody (Santa Cruz SC-335) was added to some reactions followed by $15 \mathrm{~min}$ incubation at room temperature. The samples were loaded on a nondenaturing $4 \%$ 40:1 acrylamide/bisacrylamide gel, which pre-ran for $15 \mathrm{~min}$ at $200 \mathrm{~V}$ prior to loading. Electrophoresis was carried out at $200 \mathrm{~V}$ for $25 \mathrm{~min}$, after which gels were vacuum dried and exposed to $\mathrm{x}$-ray film at $-80^{\circ} \mathrm{C}$ for various time periods.

\section{Results}

Functional analysis of the mouse SMTN-A promoter

To firmly establish that the 5' upstream region of the SMTN-A transcription start site can activate transcription in SMCs, we constructed a reporter plasmid carrying $1560 \mathrm{bp}$ of the putative mouse promoter (construct $M-1560$ ), and transfected it into PAC1 SMCs. A sixteen-fold induction of transcription compared to a promoterless construct was found, showing that this region, which is comparable to the previously tested human construct ${ }^{511}$, indeed has promoter activity in vitro (figure 5.4). To map the regions that are important for promoter activity, deletion constructs were made. Removal of nucleotides from -1315 to -1560 reduced promoter activity dramatically (construct M-1315). Upon further deletion of nucleotides from -1315 to -851 (construct $M-851$ ), activity remained low. However, 
after deletion of nucleotides between -851 and -658 (construct M-658), a strong induction of promoter activity was observed. The -208 minimal promoter fragment (construct $M-208$ ) showed a four-fold induction of transcription above baseline.

Activities of a -1616 bp SMTN-B promoter fragment and a $\alpha$-SMA promoter fragment of 195 bp were approximately six-fold and three-fold higher in PAC1 cells compared to the -1613 bP SMTN-A promoter fragment (data not shown).

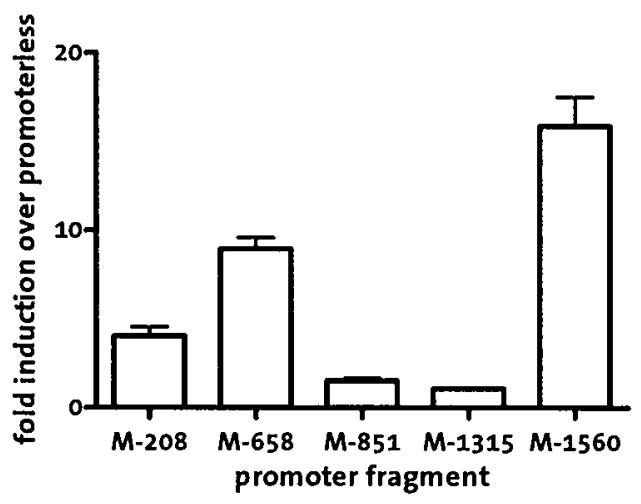

Figure 5.4 Activity of mouse SMTN-A promoter fragments of different lengths. Activities of all fragments tested differed significantly, with the exception of constructs $M-851$ and $M-1315$.

\section{Sequence analysis of CArG box-containing region}

Sequence comparisons between human, mouse, and rat (sequence obtained from GenBank accession number NW_042918.1) gave an indication about the functional significance of the CArG boxes in the SMTN-A promoter (figure 5.3). A 111 bp region in intron 8 of the smoothelin gene containing the CArG boxes showed $86.5 \%$ homology. The core of this region had only 7 mismatches in 60 bp between mouse, human, and rat. The CArG boxes themselves were completely conserved, including a $G$ substitution in the core of both CArG-far and CArG-near. The region between the CArG boxes, spanning $27 \mathrm{bp}$, showed only two non-conserved nucleotides between the three species. The 5'flanking sequence of CArG-far was conserved for three nucleotides, like the 3 'flanking sequence of CArG-near. This high degree of conservation suggests that the CArG boxes and possibly other elements in this region might be of importance for SMTN-A promoter activity.

\section{Functional significance of CARG boxes in the human SMTN-A promoter}

To investigate the contribution of the CArG boxes to the transcriptional activity of the SMTN-A promoter, we introduced mutations in each element separately, and in both elements simultaneously (figure 5.2), and assessed activity of the mutated constructs in PAC1 cells (figure 5.5). The introduced mutations are known to abolish SRF-binding to these cis-elements, rendering them inactive ${ }^{510}$. In comparison with the wild-type construct, mutation of the CArG-near box (CArGNmut) caused a significant reduction of transcriptional activity of $15 \%$ $(P=0.02)$. Mutation of CArG-far (CArGFmut) did not affect promoter activity significantly. In contrast, mutation of both CArG boxes simultaneously (CArGNFmut) reduced activity significantly by $32 \%$ compared to the wild type 
construct $(P<0.01)$. Surprisingly, co-transfection of wild type or mutated SMTN-A or SMTN-B promoter fragments with constitutively active SRF (SRF-VP16) activated these promoters only mildly in PAC1 SMCs (statistically insignificant; data not shown).

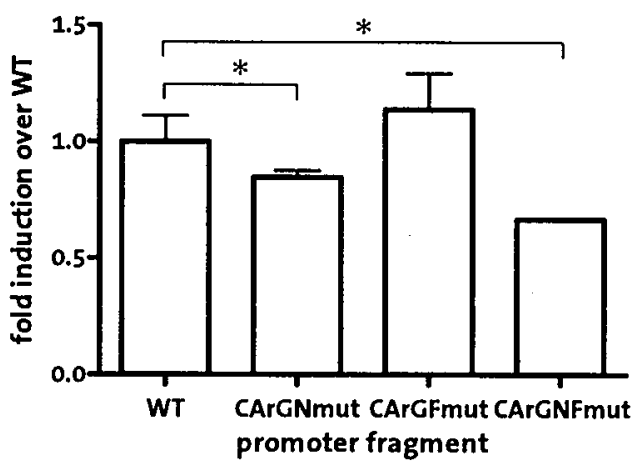

Figure 5.5 Effect of CArG box mutations on the activity of the human smoothelin-A promoter. Statistically significant differences are indicated by an asterisk.

SRF interaction with SMTN-A promoter CArG boxes

The relatively mild effect of the CArG box mutations on SMTN-A promoter activity and the minimal activation of the promoter by SRF-VP16 led us to determine if SRF was able to bind to the SMTN-A CArG boxes. To this end, we performed EMSAs using PAC1 nuclear extracts and individual double stranded oligonucleotides containing either SMTN-A CArG box and at least 6 bp of flanking sequence on each side. The results revealed several specific nucleoprotein complexes with shifted mobility for both SMTN-A probes (figure 5.6), which were competed by unlabeled wild-type probe, but not by unlabeled mutant probe. The presence of SRF in one of these complexes was demonstrated by a supershift experiment. SRF bound both SMTN-A CArG probes, although binding to CArG-near was much stronger. However, in comparison to the $\mathrm{IC}_{1} \mathrm{CArG}$ box of the SM-calponin gene, even the SMTN CArG-near bound SRF weakly. In addition, the $\mathrm{IC}_{1}$ CArG box experiment showed only one specific shifted band, representing the SRF-containing nucleoprotein complex.

Activation of the SMTN-A promoter by myocardin in a CArG-near-dependent manner To assess the ability of myocardin to transactivate the SMTN-A promoter, we performed transient reporter assays using either the wild-type promoter or the promoter with single or double CArG box mutations. Myocardin increased wildtype promoter activity 2.5-fold (table 5.1). Mutation of CArG-near greatly reduced the potential of myocardin to activate transcription (1.6-fold induction). In contrast, mutation of CArG-far did not affect myocardin transactivation significantly (2.7-fold induction). Upon mutation of both CArG boxes, the ability of myocardin to activate transcription was completely lost. SMTN-B promoter activity was augmented by myocardin, but to a lesser extent than SMTN-A. Similar to previous reports, $\alpha-S M A$ promoter activity was highly stimulated by myocardin (17.1-fold induction). 


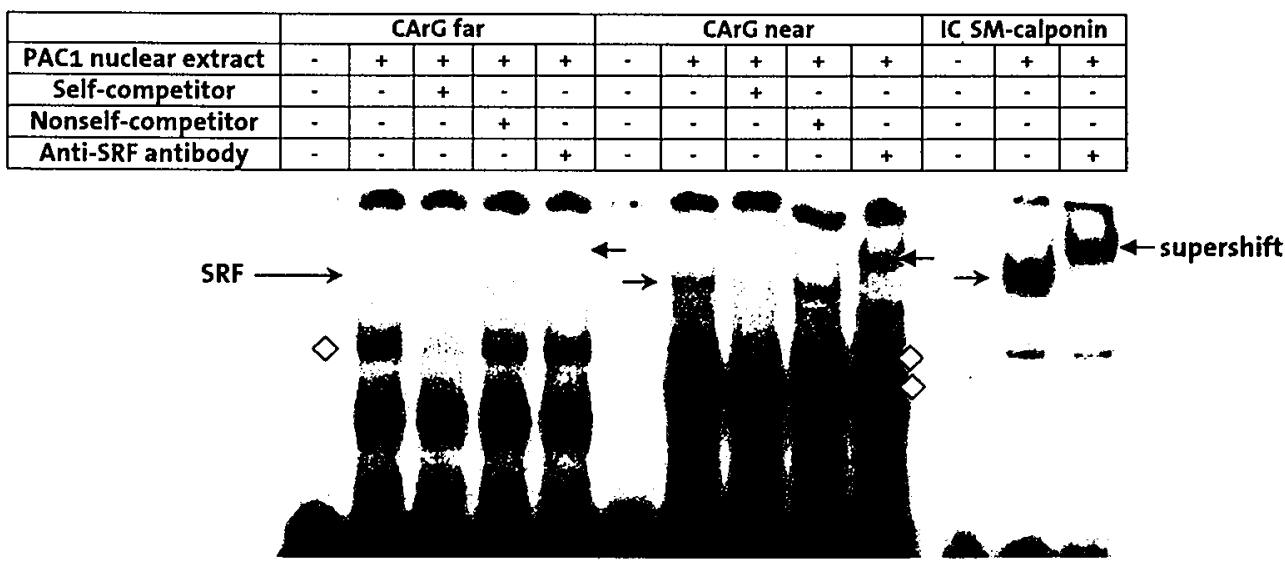

Figure 5.6 EMSA of SMTN-A and SM-calponin CArG boxes. Supershifted complexes are indicated by a left-pointing arrow, SRF bound to CARG boxes is indicated by a right-pointing arrow, additional specific complexes are indicated by diamonds. See also picture on front turnover.

Table 5.1 Effect of co-transfection of myocardin on SMTN-A, SMTN-B, and $\alpha$-SMA promoter activity.

\author{
Promoter construct \\ SMTN-A wild type \\ SMTN-A CARGNmut \\ SMTN-A CArGFmut \\ SMTN-A CARGNFmut \\ SMTN-B H-1616 \\ $\alpha$-smooth muscle actin
}

\section{Fold induction}

$2.5 \pm 0.1$

$1.6 \pm 0.2$

$2.7 \pm 0.5$

$0.9 \pm 0.1$

$1.7 \pm 0.2$

$17.1 \pm 2.3$

\section{Discussion}

SMC differentiation is monitored by marker genes whose expression is restricted to SMCs and representative of a given stage of differentiation. In recent years, it has become clear that studies on the regulation of marker gene expression can direct us to those cis-elements and trans-factors that are important for SMC differentiation itself. So far, the recurring theme in this type of studies is the critical role of SRF-binding to one or more CArG elements in the promoters of SMC marker genes $^{268.512}$. Many of these genes are downregulated when SMCs modulate towards a synthetic phenotype. The extent of downregulation is however quite variable between the different markers. The gene that seems to be most responsive to conditions promoting the synthetic phenotype is the SMTN gene ${ }^{79,83}$. Elucidation of mechanisms that regulate the expression of this gene is therefore likely to contribute to the identification of regulators of the contractile SMC phenotype. 
Like several other genes whose expression is restricted to SMCs, the SMTN gene generates several isoforms. Unlike these other genes, this is the result of a dual promoter system, instead of alternative splicing. An intragenic promoter fragment which resides in a region where several exons of SMTN-B are located has been shown to drive transcription of the smaller SMTN-A mRNA ${ }^{511}$. Data presented in this study confirm and extend these results by showing that although SMTN-B promoter activity is higher, several SMTN-A promoter constructs were consistently active in PAC1 SMCs. Detailed investigation of the mouse SMTN-A promoter by deletion constructs revealed a rather complex pattern of regulation. The promoter appeared to contain both strong repressing and strong enhancing elements. The region between -1315 and -1560 bp contains several conserved E-boxes, ciselements that have been shown to enhance $\alpha$-SMA promoter activity ${ }^{302}$. Even though they are present in sequences coding for SMTN-B, these E-boxes may be involved in positive regulation of SMTN-A transcription. Interestingly, a cis-element in the first exon of the SM22 $\alpha$ gene was recently demonstrated to contribute to SM $22 \alpha$ expression ${ }^{298}$, showing that coding and regulating functions of the same sequence are not mutually exclusive.

Apart from the E-boxes, the tested SMTN-A promoter fragments also contain two conserved CArG elements in close proximity in an intronic region ${ }^{501}$. In this study, the functional significance of these elements has been tested. We have shown that the CArG boxes in the SMTN-A promoter contribute to, but are not crucial for SMTN-A promoter activity. This is in marked contrast to the promoters of other SMC restricted genes with paired CArG boxes like $\alpha$-SMA and SM $22 \alpha$, which show $80 \%$ reduction of activity when either of their CArC boxes are mutated ${ }^{252,500}$. The discrepancy of these findings may be explained by the differential organization of the CArG elements between these genes, for it is known that CArG spacing and phasing have a major impact on promoter activity ${ }^{267}$. The distance between the CArG boxes in the $\alpha$-SMA and SM22 $\alpha$ genes is 40 and 113 bp respectively, as compared to the SMTN-A CArGs that are only 27 bp apart. This may lead to different binding potential of SRF or SRF-cofactors. Apart from this, particularly CArG-far has a relatively low affinity for SRF in EMSA experiments. CArG-near has a higher SRF-binding potential, which is in line with data on the affinity of SRF for CArG boxes with different $G / C$ substitutions in the core, which indicate that a mutation at the last position of the AT core (CArG-near) is better tolerated than a mutation in the middle (CArG-far) ${ }^{268}$. Thus, it may be that only one of the SMTN-A CArG elements (CArG-near) is a functional SRF-binding element, contributing to SMTN-A promoter activity. Alternatively, SRF binding to CArG-near may be required to facilitate SRF binding to CArG-far, like has been shown for the skeletal actin promoter $^{513}$.

The finding that specific nucleoprotein complexes with either SMTN-A CArG box were formed in EMSAs which could not be supershifted by our SRF antibody could 
have several implications. It may indicate that an alternative isoform of SRF is involved in this binding ${ }^{271}$, which is not recognized by the SRF antibody we employed. Alternatively, it may represent an SRF-cofactor complex of unknown nature, in which the cofactor masks the epitope recognized by our SRF antibody. Another possibility would be that distinct proteins unrelated to SRF bind these sequences. Interestingly, the interaction between these putative unknown factors and the SMTN-A CArG probes appeared to be more robust than the interaction with SRF. If these factors actually regulate SMTN-A promoter activity, they might be negative in nature, since the $M-1315$ promoter fragment containing the CArG probe region was not active in PAC1 cells, and mutation of CArG-far actually increased promoter activity, albeit not significantly. In this respect, it is noteworthy that the YY1 transcription factor can antagonize SRF binding to CArG boxes, thereby reducing SM22 $\alpha$ promoter activity ${ }^{514}$. A similar way of regulation may be implicated here.

Collectively, these results show that although SRF binding to the SMTN-A CArG boxes is functional, this is not essential for SMTN-A promoter activity. This suggests that the transcriptional activation of SMTN-A, which is associated with the advanced contractile SMC phenotype, requires additional factors.

In view of the recently suggested model in which myocardin specifically enhances transcription of genes containing two CArC boxes ${ }^{279}$, we tested the ability of myocardin to increase transcription of SMTN-A and -B. SMTN-B promoter activity was only marginally induced by myocardin, which probably relates to the absence of a conserved CArG element in its promoter. In contrast, myocardin did activate the SMTN-A promoter, albeit to a much lesser extent than the $\alpha$-SMA promoter. In agreement with the data on SRF binding, CArG-near but not CArG-far appeared to be important for SMTN-A promoter activation by myocardin. Myocardin activity was completely lost when both CArGs were mutated. Since myocardin acts exclusively via SRF bound to CArG boxes, this confirms that the SMTN-A CArG boxes are functional. Recently, similar results were obtained in a study of the $\alpha$-SMA promoter, although mutation of only CArG-far severely impaired promoter activation by myocardin ${ }^{242}$. This suggests that activation of promoters in SMCs by myocardin is not absolutely dependent on two functional CArG motifs, but rather that one intact CArG element is sufficient. However, myocardin transactivation of the SMTN-A promoter in PAC1 cells is much weaker than observed for other SMC genes ${ }^{39}$, probably because the SMTN-A CArG boxes do not bind SRF very strongly. Thus, the hypothesized mechanism in which myocardin's transactivational activity is only fully revealed after bridging of two CArG-bound SRF molecules is consistent with the present data ${ }^{279}$. It may also be that another factor, possibly a myocardin related molecule like MRTF- $A^{280}$, is needed for full activation of the SMTN promoters. 
It is important to note that results obtained by in vitro studies on the regulation of $S M C$ promoters, including those of SM22 $\alpha$ and $\alpha$-SMA, are often not confirmed in vivo ${ }^{253.261}$. However, the inconsistency mostly concerns a CArG box that was shown to be sufficient for promoter activation in vitro but not in vivo, where it required additional elements for promoter activity. Therefore, the involvement of the CArG elements in expressional regulation of SMTN-A needs in vivo confirmation using transgenic mice. Moreover, the possibility that the stronger upstream SMTN-B promoter is required for full exploitation of the SMTN-A promoter cannot be ruled out at present, and would provide a novel contribution to the complex ways in which expression of SMC genes is regulated.

\section{Acknowledgements}

This work was supported by National Institutes of Health grant HL-62572. We would like to thank Dr. Eric Olson for the generous gift of the mouse myocardin expression plasmid. 


\title{
Structure and chromosome location of Smtn, the mouse smoothelin gene
}

\author{
Sander Rensen*, Gerard Merkx**, Pieter Doevendans s**, $^{* *}$ \\ Ad Geurts van Kesse ${ }^{* *}$, Guillaume van Eys*
}

- Department of Genetics and Cell Biology, Cardiovascular Research Institute Maastricht, Universiteit Maastricht, Maastricht, The Netherlands

** Department of Human Genetics, University Hospital, Nijmegen, The Netherlands

*** Department of Cardiology, Heart Lung Center Utrecht, The Netherlands / Interuniversity Cardiology Institute, Utrecht, The Netherlands

Based on: Cytogenet Cell Genet 2000; 89: 225-229 


\section{Abstract}

Smoothelins are cytoskeleton-associated proteins that are found in contractile smooth muscle. Two isoforms have been identified: smoothelin-A, expressed in visceral tissues and smoothelin-B, found in vascular tissues. The mouse smoothelin gene (Smtn) was isolated and characterized. It was assigned to chromosome 11 band A2-A3 by fluorescence in situ hybridization. The gene consists of 21 exons and spans $23 \mathrm{~kb}$. Its structure is conserved between mouse and human. The proximal promoter of both smoothelin-A and smoothelin-B contains several transcription factor-binding sites, but lacks a consensus TATA box.

\section{Introduction}

Smoothelins are cytoskeleton-associated proteins of unknown function that are specifically expressed in contractile smooth muscle cells (SMCs) ${ }^{23}$. In humans, two isoforms have been described: smoothelin-A, which is predominantly expressed in visceral SMCs, and smoothelin-B, which is found in vascular SMCs ${ }^{80}$. Smoothelin-A has a molecular weight of $59 \mathrm{kDa}$, whereas the vascular smoothelin-B is a $110-\mathrm{kDa}$ protein. Smoothelin-A is equal to the C-terminus of smoothelin-B. The $\mathrm{N}$-terminus of smoothelin-B thus accounts for the increased molecular weight of the protein. Both proteins have a potential actin-binding domain, which is homologous to that of proteins of the spectrin-family. The subcellular localization of smoothelin-B has been investigated in cultured human, porcine, and chicken SMCs, showing that smoothelins colocalize with $\alpha$-smooth muscle actin ${ }^{23,80,83}$. Smoothelins are predominantly found in contractile smooth muscle tissue. They are not expressed in SMCs of diseased tissues such as aneurysms ${ }^{79}$. In addition, their expression decreases progressively in cultured $\mathrm{SMCS}^{23,80}$. All these data suggest a role of smoothelin in the contractile apparatus of SMCs. Human smoothelin-A and -B are coded for by a single copy gene, located on chromosome $22 q 12.3^{433}$. These two isoforms are generated by a dual promoter system ${ }^{511}$.

Insight into the structure of the mouse smoothelin gene is essential for further physiological and biochemical studies by gene targeting. The sequence of the mouse CDNA for smoothelin-B has been described by Krämer et al. ${ }^{81}$. The 3142-bp CDNA showed $80 \%$ homology to the previously described human smoothelin-B CDNA sequence. Here we report on the chromosome location and exon/intron organization of the mouse smoothelin gene. The smoothelin gene structure and promoter sequences of human and mouse are compared. 


\section{Materials and methods}

\section{In situ hybridization}

The chromosome location of the mouse smoothelin gene was determined by fluorescence in situ hybridization (FISH) on mouse metaphase spreads essentially according to Suijkerbuijk et al. ${ }^{515}$. In brief, a cosmid containing the entire mouse smoothelin gene, and mouse whole chromosome 11 painting probes were labeled by nick translation with biotin-14-dATP (Life Technologies, Gaithersburg, MD) and digoxigenin-11-dUTP (Boehringer Mannheim, Almere, The Netherlands) respectively, and ethanol precipitated together with 50-fold excess of mouse Cot-1 DNA (Life Technologies). Subsequently, $200 \mathrm{ng}$ cosmid DNA and $400 \mathrm{ng}$ whole chromosome 11 paint DNA were dissolved in a total volume of $12 \mu$ l hybridization mixture (containing formamide, dextran sulphate, and Tween-20). This mixture was heat-denatured and then incubated overnight at $37^{\circ} \mathrm{C}$ with heat-denatured metaphase spreads under a cover slip. Immunocytochemical detection of the hybridizing probes was achieved using fluorescein isothiocyanate (FITC)conjugated avidin followed by two amplification steps with rabbit-anti-FITC and mouse-anti-rabbit-FITC and sheep-anti-digoxigenin conjugated rhodamine and monkey-anti-rhodamine conjugated Texas Red. Images were captured using a Zeiss epifluorescence microscope and a cooled CCD camera (Photometrics, Munich, Germany).

\section{Cloning and sequencing}

To determine the exon/intron structure of the mouse smoothelin gene, a genomic mouse cosmid library was screened with ${ }^{32}$ P-labeled human smoothelin-B CDNA. Clones hybridizing to the probe were selected and purified ${ }^{516}$. The clone containing the largest insert was characterized by restriction mapping and Southern blotting, which enabled the subcloning of exon containing fragments of the gene into pBluescript SK (Stratagene, La Jolla, CA). Sequencing of these subclones was done with an $A B 1310$ automatic sequencer (Perkin Elmer, Norwalk) after cycle sequencing with either M13 or smoothelin-specific primers. Subsequently, the exons were positioned by comparing cDNA and genomic sequences. Homology searches were performed using $\mathrm{BLAST}^{496}$, whereas detection of potential exons in intron sequences was done with HEXON software ${ }^{517}$. The MatInspector Professional computer program was used to screen promoter sequences for promoter/enhancer elements ${ }^{518}$.

\section{Results and discussion}

Chromosome location of the mouse smoothelin gene

FISH on mouse metaphase spreads with a smoothelin cosmid probe revealed a clear signal only on chromosome 11 in all metaphases examined (figure 6.1a). Co- 
hybridization with the whole chromosome 11 painting probe unequivocally confirmed this observation. Thus, the mouse smoothelin gene is a single copy gene mapping specifically to mouse chromosome 11A2-A3 (figure 6.1b). This assignment agrees with the known synteny between human chromosome $22 \mathrm{q} 12$ and mouse chromosome $11^{\text {s19. }}$.
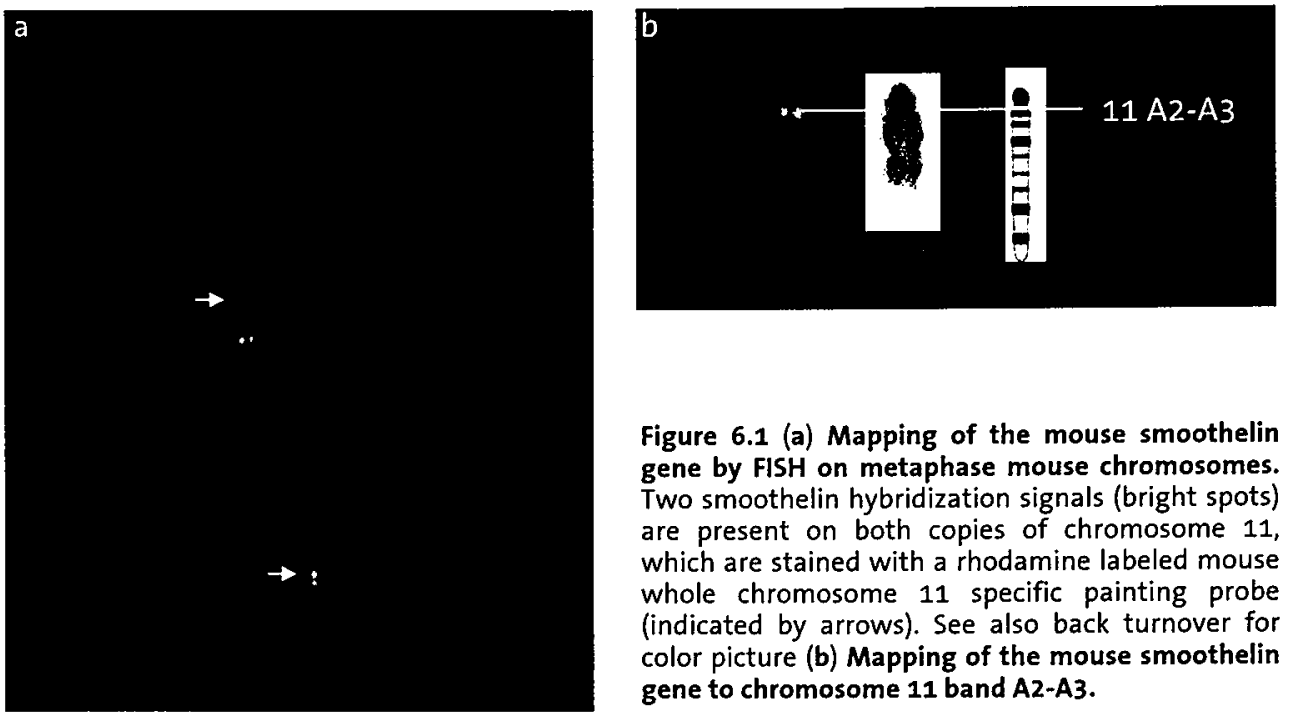

Figure 6.1 (a) Mapping of the mouse smoothelin gene by FISH on metaphase mouse chromosomes. Two smoothelin hybridization signals (bright spots) are present on both copies of chromosome 11, which are stained with a rhodamine labeled mouse whole chromosome 11 specific painting probe (indicated by arrows). See also back turnover for color picture (b) Mapping of the mouse smoothelin gene to chromosome 11 band A2-A3.

\section{Mouse smoothelin gene structure}

The mouse smoothelin gene consists of 21 exons, varying in size from 62 bp (exon 15) to $516 \mathrm{bp}$ (exon 10) (figure 6.2). Exon 1 and the $5^{\prime}$ part of exon 2 contain the $5^{\prime}$ untranslated region of smoothelin- $B$, whereas a part of exon 10 contains the $5^{\prime}$ untranslated region of smoothelin-A. The $3^{\prime}$ untranslated region of both transcripts is confined to the $3^{\prime}$ part of exon 20 . In comparison with the human gene, most introns are smaller. This results in a total size of the mouse gene of 23 $\mathrm{kb}$ as compared to $25 \mathrm{~kb}$ for the human gene. The overall structure of the mouse gene with respect to spacing of the exons is similar to the human gene, indicating a rather strict evolutionary conservation. Introns 2, 11, and 19 are large, between 3 and $4 \mathrm{~kb}$. Exons 4-6, 7-11, 12 and 13, 14-16, and 17-19 are clustered in both genes. All exon/intron boundaries of the mouse smoothelin gene are in accordance with the GT/AG rule (GenBank accession numbers AF116516 - AF116518, AF195524, AF195525, AF218829 - AF218834). BLAST computer analysis revealed that certain sequences in intron 11 and 19 were conserved between human and mouse. Further analysis by HEXON software predicted two additional exons in both introns. Interestingly, the two putative exons in intron 11 are situated exactly in a conserved region. However, cDNAs incorporating these putative exons have not been found so far. In contrast, a novel cDNA that contains an extra exon of $165 \mathrm{bp}$ in intron 19 has recently been found in human prostate tumor cell lines ${ }^{506}$. This 
exon was accurately predicted by HEXON, and is conserved between mouse and human. The amino acid sequence of exon 19 and exon 20 shows $45 \%$ homology to 86 amino acids of the actin-binding domain of proteins of the spectrin family as shown by BLAST analysis. The previously described smoothelin-A isoform contains only 55 homologous amino acids (exon 19) ${ }^{23}$. The presence of a larger part of the actin-binding domain may increase the ability of the alternatively spliced isoform to bind actin. Exon 20 introduces a different termination site, making the actinbinding domain the C-terminal end of the protein.

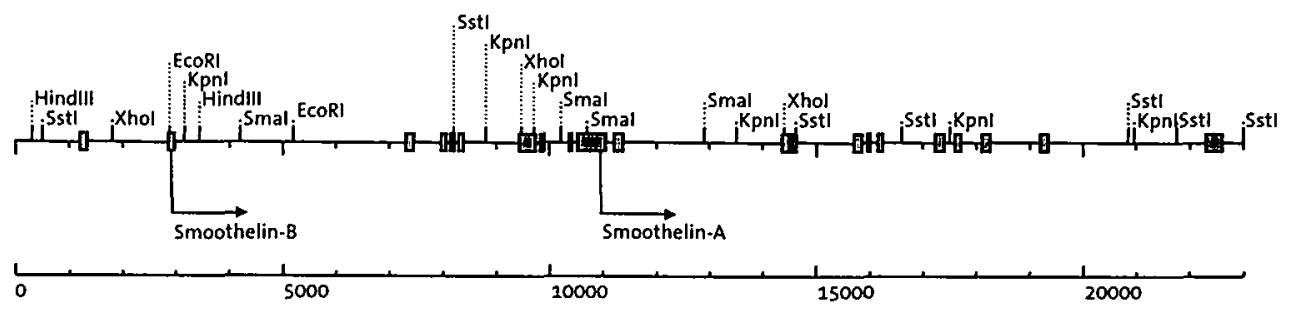

Figure 6.2 Exon/intron organization of the mouse smoothelin gene Smtn. Exons are depicted by filled boxes. A number of restriction sites used in the analysis are shown. Putative translation initiation sites of smoothelin- $A$ and $-B$ are indicated by arrows.

\section{Smoothelin promoter analyses}

The sequence of about $730 \mathrm{bp}$ upstream of the first mouse smoothelin-B exon was compared to the same region of the human smoothelin-B gene (figure 6.3a). Although the overall homology of this region is $66 \%$, sequences that are $90-100 \%$ conserved can also be identified. Such sequences may be involved in the regulation of smoothelin gene expression. Analysis of the promoter sequence revealed a number of cis-acting elements, although a TATA box was not detected. This is rather uncommon for smooth muscle specific genes ${ }^{253.256,520}$. In view of the important role of CArG boxes in the regulation of several smooth muscle-specific genes, it is interesting that a conserved CArG-like box is present at position - 627 . Additional non-conserved CArG boxes are found at -281 in the mouse promoter and at -79 and -499 in the human promoter. Furthermore, a number of conserved Sp1 $(-49,-135)$ and AP $(-113,-378)$ sites are located in the smoothelin-B promoter region. The presence of an entirely conserved consensus MyoD binding site at position -448 bp may be relevant, since smoothelin is expressed in early embryogenesis in the somites and the primitive heart ${ }^{521}$. MyoD is also expressed in those tissues at that time and may be involved in the regulation of smoothelin expression in embryogenesis ${ }^{522}$. Additional elements identified in the smoothelin-B promoter include relatively conserved NF-1 (-250), C/EBP $\alpha$ (-454 and -663$), \mathrm{Pbx}-1 \mathrm{a}$ (-543), PADS-C (-359), and gut-enriched Krüppel-like factor (-344) sites.

The sequence of about $1750 \mathrm{bp}$ of the proposed smoothelin-A promoter has been determined for both mouse and human (figure 6.3b). Exons 7 through 10 of smoothelin-B are positioned in this region. The overall homology in this region is $73 \%$, while intron sequences are as conserved as exons 7 through 9 . Inside intron 8 , 
CTCACCTCTTGGGTCTCCTCGTTGACTCTTTGT-ATTG-TCTC-CTTGGCATCTTCA

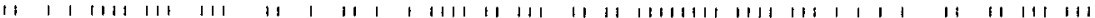

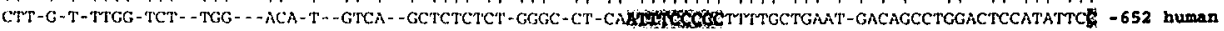
C/BBPQ

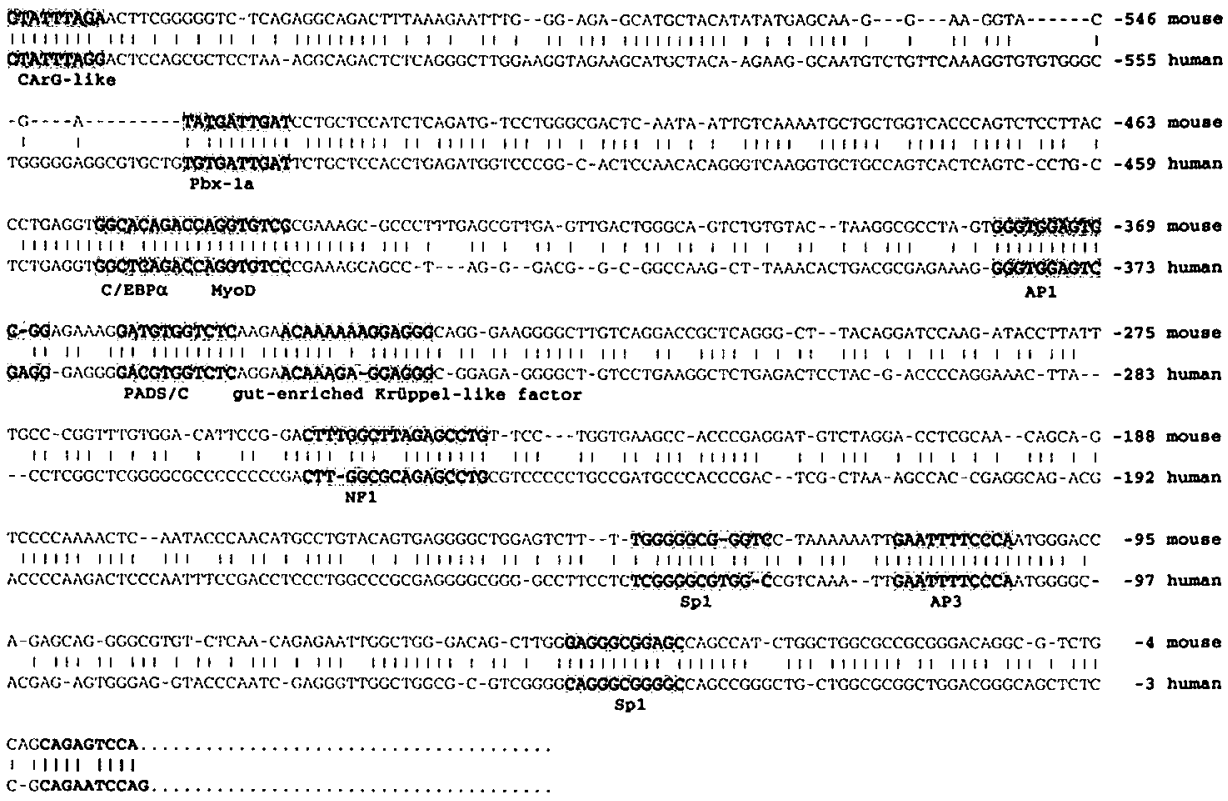

Figure 6.3 (a) Homology between mouse and human smoothelin-B promoter region. Several cisregulatory elements are indicated. (b (next page)) Homology between mouse and human smoothelin-A promoter region. Conserved cis-regulatory elements are indicated.

a section of 111 bp showing $90 \%$ homology is present, which contains two CArC boxes in close proximity. Again, no TATA box was found. Nevertheless, a number of described regulatory elements were recognized in the mouse smoothelin-A promoter. Among them are MyoD sites, Sp1 sites, one NFKB site, a myogeninbinding site, one AP4 site, and a glucocorticoid responsive element. Most of them are conserved between mouse and human. Although the regions described here have been shown to display promoter activity in several cell lines, the functional significance of the identified regulatory elements will need further study. In conclusion, the smoothelin gene shows a strong conservation between mouse and human in its chromosome location, its organization, and its exon and promoter sequences. The degree of conservation supports the previously suggested importance of the gene.

\section{Acknowledgements}

The authors would like to thank Prof. Dr. B. Wieringa (University of Nijmegen) for providing the genomic cosmid library. The chromosome 11 mouse paint was kindly provided by Dr. Coco Martin (NKI Amsterdam). 


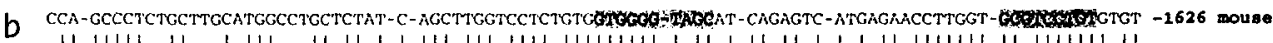
$111111111 \quad 1111 \quad 1111111111111 \quad 1111111111111111111111111111111111111111111111$

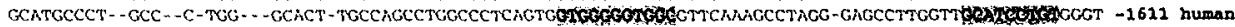
sp1 c-Ets1

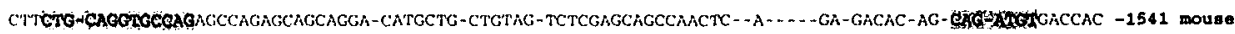

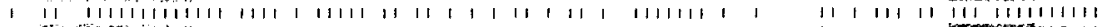

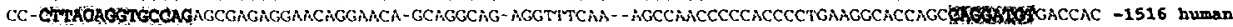
MyoD

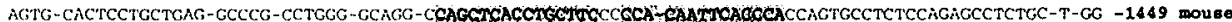

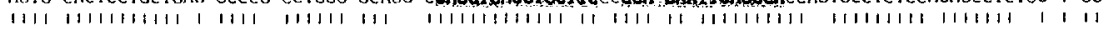

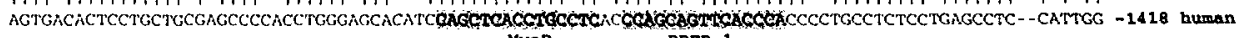
MYOD RREB-1

AGCCTGCTGAG-CC-ATGTCTCCTG-C'TG-TGAGGCTCCAGTCAGCTCTGAGCCACTFCCACA-CCCTICAGMAG-CT-CCATGCCCTGAGCCCCCC-NT -1357 mOUBE

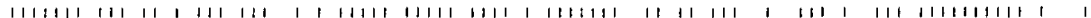

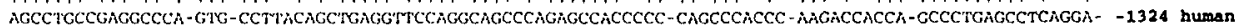

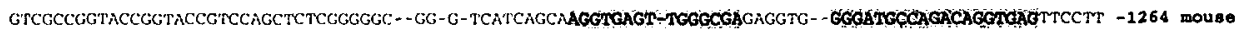

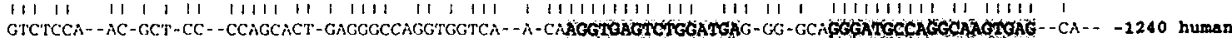
Thingl/E47 NFKB (Y)

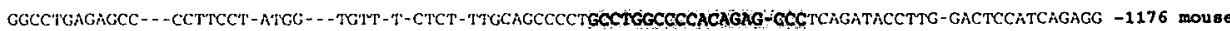

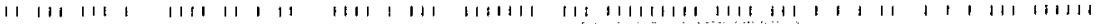

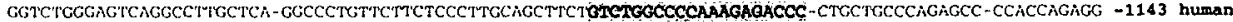
$s p 1$ RPBB-1

COCTCCACRCIMAGAGAGCAGGTAGGAÖ-CCCTGTTRCAGCT-TAC-CACAGGCTICTGCCTTCTCCTGTCCTGTCCCAT-C-TCCCCTTTCCCTG - 2081 mOUBE

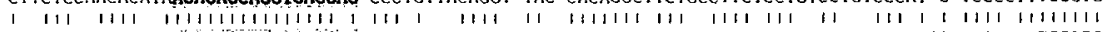
CCCCTCTYACACC-AAGAGAGCAGGTGAGG-GTCCCAG--CAGCGGTAGTCACAGGCATCTTTCTTCCCCT--CC---CCC-TGCCTCCC-TTTCCCTG - 1055 nUman MYoD

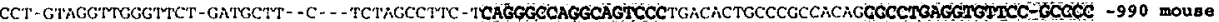
$111111111111,111,11111111111111111111111111111111111111111111111111111$

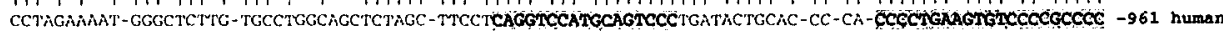
PacC $/ L Y-A$ I c-Eta-1 $\quad$ spi

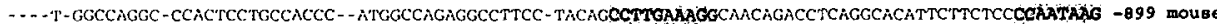
1111111 1 1 1 1 11111 11111 1111111111 1 11111111111111111 1 1111111111111111111111111111 CAGCPACGCCAGCCTCC-C-CCT-CCACCCCCATGCCTAGAGGCCTCCCCTGCAGCOTIGBÄBGGCAACGGGCCTCAGGCACATTCTTCTCCCOAATAAO -864 hUman CArG-11ke

CAYG-1ike

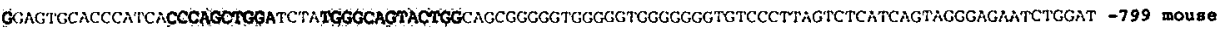
$11111111111111111111111+1111111111$ 1 11 1 11 11 11111111111111111111

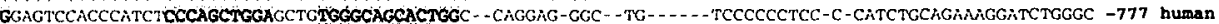
AP4 SRY- $\beta$

ACCTTTC-TCTGCCCCTAA-CTTACTTCTEGCCCCLAAGATCCCCACGTCACCCC-GGGCCCGCGGTCAGTGCAGGAGAGMAGGAGGT-CCTTGTAGG -703 mOUBE

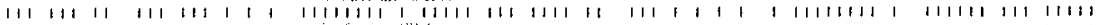
ACCCTICCTCCAGCC-TAGCCT-CGCCTGGCCCCCAGGAGTGTCCAMTCATCCTAGCGTCAGTG-T--G-GCAGGAGA-ñ--GGAGCTGCCT-GTAGC -687 hURAR AP2

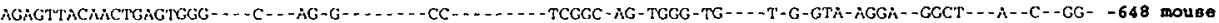

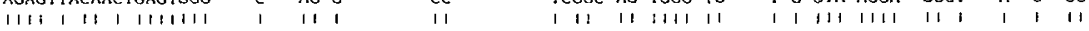

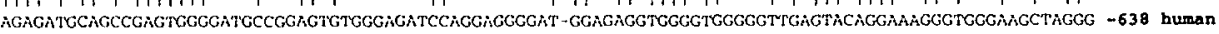

AGGTGTTGAGGÄRGG -TGAGTG---GCGTGAACACCATCCCACCATCCACTTTCCTTTC-CTCTCCCCCCATCCCCCTTATGGCTACCACATACTLACC -553 DOUQE

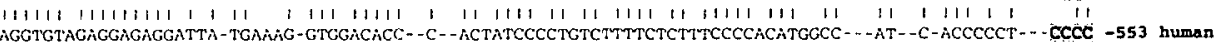

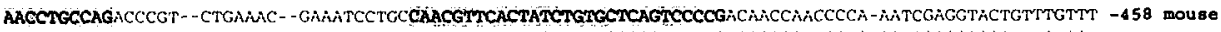

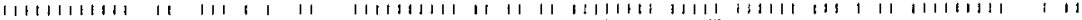

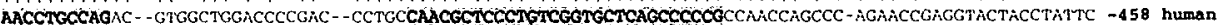
RREB-1 Byogen I INFI

TTTTTTGACCATCCCTAGGTTCTGACACTCCCTATCCCACCCAGCCACTGACAGCTTTCTTGTCTCCTCC-AGAG-CCANCCTCACTTGC-AG-G-AC -363 mOUBE $1 \quad \mid 11111111111111111111111111111111111111111111111111111111111111111$

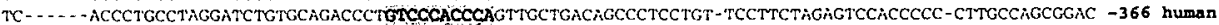
SP1/NFKB

CGTCC-CAGTTCC-GTCEAGTTGGCTCTGTGAGAGA-GAGAGTOCAAAAGTTCACATCTGATTCTCCCCTGGTTGCCAGGCTCCAGGATGGCCCACCCCG -266 mOU Be 1111111111111111111111111111111111111111111111111111111111111111111111111111

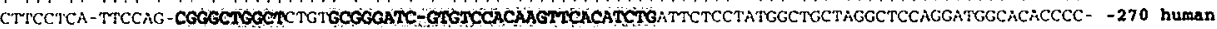
Spl Hyogen in/NP1

AA-CACCCCTT-GCTTCACC-GrCCCCCACAMGCTCCCGGGCCCACCCHCATCAGCACCACCOCTGCCTCCTCCTCCTCCAGCARCTCCTCCTCTCCG -169 mOU Se

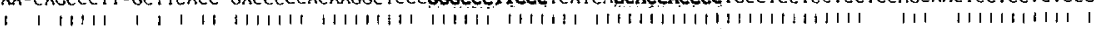

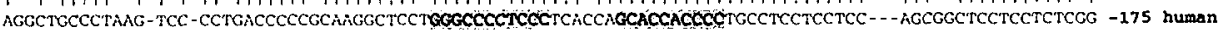
C-Rel Sp1

AGTCCCAGTGACACTTCCTCCC - . - A-CAMGMGCAGA-GAGALCTTGCTCATTCCCTGGCCGAGCTTCAGA-GCTGCCCTCAAGAGGAGGCCCTGG -77 DOUBE

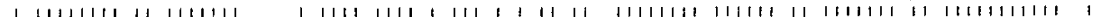

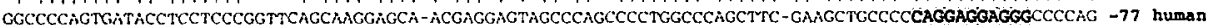
SD1 C/EBPa

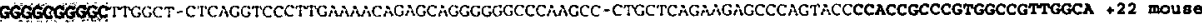

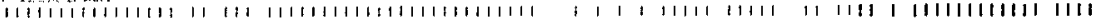
GG GGGGGGCTGGCTGCT-AGGCCCCTTGWWACAGAGCAGGGGGGCCTGTGGCAC-GTTCAGAGGAGCCTGGTGCCCCGCTGCCCGTGGCGTCGGCA + 22 human spl 



\title{
Smoothelin deficiency leads to lethal smooth muscle cell dysfunction in mice
}

\author{
Sander Rensen*, Petra Niessen*, Jan van Deursen**, Marion Gijbels*, \\ Pieter Doevendans ${ }^{* * *}$, Jo De Mey ${ }^{* * *}$, Marten Hofker*, Guillaume van Eys* \\ - Department of Genetics and Cell Biology, Cardiovascular Research Institute Maastricht, \\ Universiteit Maastricht, Maastricht, The Netherlands \\ ** Department of Pediatrics and Adolescent Medicine, Mayo Clinic, Rochester, MN, USA \\ *** Department of Cardiology, Heart Lung Center Utrecht, The Netherlands I \\ Interuniversity Cardiology Institute, Utrecht, The Netherlands \\ ..." Department of Pharmacology and Toxicology, Cardiovascular Research Institute Maastricht, \\ Universiteit Maastricht, Maastricht, The Netherlands
}




\section{Abstract}

Smoothelins are contractile smooth muscle cell-specific proteins that bind actin. We have generated smoothelin knockout $\left(\mathrm{SMTN}^{-1}\right)$ mice to investigate the function of this protein. SMTN ${ }^{-/}$mice can live up to four months, although $~ 50 \%$ die neonatally. SMTN ${ }^{-1}$ mice show reduced bodyweight and are infertile. They display pathologies in various smooth muscle tissues. The intestines contain diverticula and intestinal function in vivo is diminished. Hydronephrosis and cholestasis develop frequently. Vascular smooth muscle structure and contractile function is compromised. Our results demonstrate an essential role for smoothelins in the contraction of smooth muscle.

\section{Introduction}

The most important function of smooth muscle cells (SMCs) is contraction. By generating coordinate contractions and subsequent relaxation, they maintain blood flow and pressure, and take care of peristalsis and excretion.

At the molecular level, SMC contraction is still incompletely understood. As in skeletal muscle, the basic mechanisms of myosin and actin filament sliding to generate force apply, but the regulation and mechanics of the contraction are distinct. This can be partly explained by the presence of SMC-specific contractile protein isoforms $\mathrm{s}^{523}$. In addition, although SMCs contain tropomyosin, the complementing troponin system to confer $\mathrm{Ca}^{2+}$ sensitivity to the contraction is absent ${ }^{354}$. Instead, SMCs contain unique contractile filament-associated proteins like heavy-caldesmon, SM-calponin, and telokin which take part in the regulation of SMC contraction ${ }^{5}$.

The function of smoothelin, another SMC-specific actin filament accessory protein ${ }^{23}$, is still unclear. Smoothelin occurs as a B-isoform, which is predominantly expressed in vascular SMCs, and a smaller A-isoform, which is primarily expressed in visceral $S M C s^{511}$. Previously, we have proposed that smoothelins might be involved in SMC contraction, based on their direct interaction and colocalization with $\alpha$-smooth muscle actin $(\alpha-S M A)^{23,83,504}$, and the fact that their expression highly correlates with the contractile phenotype of SMCs and with the nature of contraction ${ }^{79,82}$. To investigate the role of smoothelins in SMC function and more in particular in contractile performance, we have created a mouse model with a disrupted smoothelin (SMTN) gene. We report that absence of both smoothelin isoforms is lethal. Our results show that smoothelins are essential for smooth muscle contractile performance, and identify smoothelins as proteins that are structurally involved in SMC contraction. 


\section{Materials and methods}

\section{Generation of SMTN" mice}

Cloning and sequencing of the mouse SMTN gene has been reported previously ${ }^{501}$. To mutate the SMTN gene, part of exon 18, exon 19, and exon 20 were replaced with a neomycin gene under the control of the thymidine kinase promoter (tk-Neo) in reverse orientation (figure 7.1a). These exons encode a calponin homology domain involved in the actin-binding properties of smoothelins ${ }^{504,524}$. The targeting vector also contained a thymidine kinase gene under the control of the phosphoglycerate kinase promoter (pgk-TK) for negative selection. Genomic fragments of 3.0 and $1.8 \mathrm{~kb}$, flanking exons 18 and 21 , were cloned into the targeting vector. The Pvull-linearized construct was electroporated into mouse L129/SVJ embryonic stem cells (ES cells). After 1 day, neomycin-resistant clones

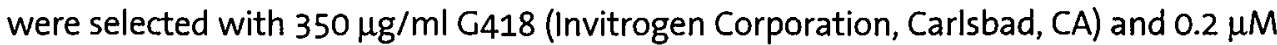
1-[2-Deoxy]2-fluoro- $\beta$-D-arabinofurasonyl (Invitrogen Corporation) for 9 days. DNA from resistant clones was screened by Southern blotting after Pstl digestion using the 3'probe indicated in figure 7.1a. ES cells from two independently targeted clones were injected into $\mathrm{C} 57 \mathrm{Bl} / 6$ blastocysts and implanted into pseudopregnant C57Bl/6 females. The resulting chimeric males were mated to $\mathrm{C} 57 \mathrm{Bl} / 6$ females and germline transmission of the targeted allele was detected by Southern blotting (figure 7.1b) and confirmed by polymerase chain reaction (PCR)-based genotyping. Since the mice had a mixed background ( $L 129 / \mathrm{SV}$ and C57Bl/6), littermates were used for experimentation whenever possible. In rare cases, age and sex matched controls were used. All animal experimentation was performed according to protocols approved by the Committee on Animal Experimentation of the University of Maastricht.

Reverse transcriptase-polymerase chain reaction (RT-PCR)

Total RNA was extracted from various tissues with Tri reagent (Sigma-Aldrich, Zwijndrecht, The Netherlands) according to the manufacturer's instructions. Reverse transcription was performed using $2 \mu \mathrm{g}$ of RNA, oligo(d)T primers, and Superscript II reverse transcriptase (Invitrogen, Breda, The Netherlands). Expression of SMTN mRNA was investigated by PCR using the following primers covering the CDNA (see figure 7.1a): 1F 5'CCAGGGGGCAGTATGAAGAC, 1R 5'CGCAGGTGGTTGTA GAGCGA \& 2F 5'GAGGAGCGCAAGCTGATCA, 2R 5'CTGCTGGTGCTGAGAAGGGT). PCR parameters were $94^{\circ} \mathrm{C}$ for $5 \mathrm{~min}$, followed by $35 \mathrm{cycles}$ of $94^{\circ} \mathrm{C}$ for $30 \mathrm{sec}, 60^{\circ} \mathrm{C}$ for $30 \mathrm{sec}, 72^{\circ} \mathrm{C}$ for $1 \mathrm{~min}$, and a final step of $72^{\circ} \mathrm{C}$ for $5 \mathrm{~min}$. RT-PCR products were cloned into the PGEM-T easy vector (Promega, Madison, $\mathrm{WI}$ ) and sequenced.

\section{Immunohistochemistry}

Femoral arteries were snap-frozen in liquid nitrogen-pre-cooled isopentane and embedded in OCT Tissue Tek compound (Sakura, Chicago, IL). Cryostat sections were stained with FITC-labeled mouse monoclonal antibody against $\alpha$-SMA (Sigma- 
a

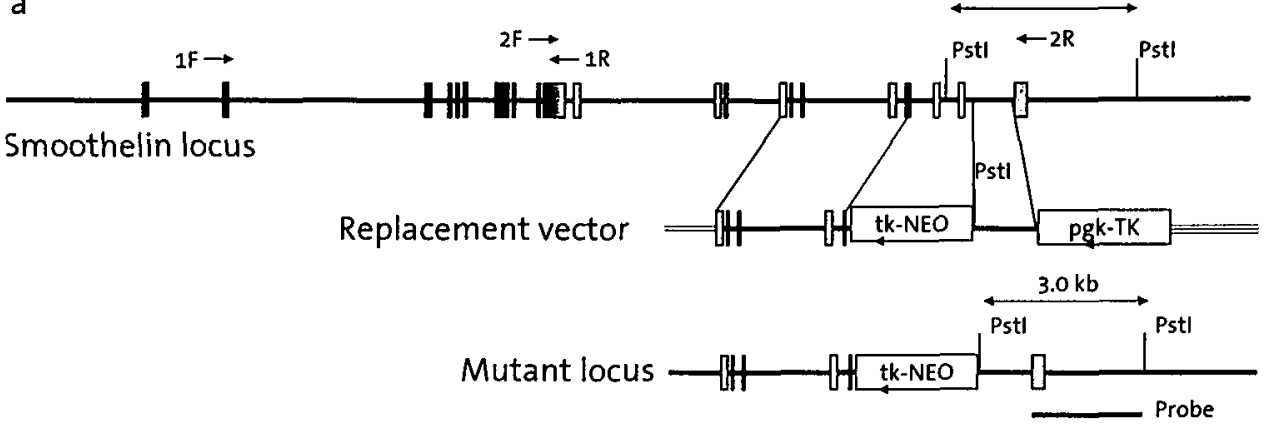

b

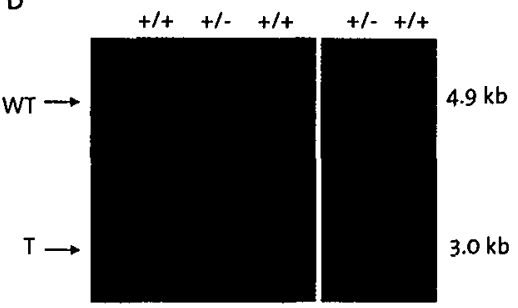

Figure 7.1 Targeted disruption of the SMTN gene. (a) Schematic representation of the targeting strategy for the SMTN gene. Top: Structure of the SMTN gene. Dark grey boxes indicate smoothelin-B exons, light grey boxes indicate exons common to smoothelin-A and $-B$, white boxes represent targeted exons. Middle: SMTN targeting vector containing neomycin resistance (tk-Neo) and herpes simplex virus thymidine kinase (pgk-TK) genes, both transcribed in the reverse direction to that of the SMTN gene, as indicated by arrows. Bottom: Structure of the targeted SMTN allele and location of the probe used in Southern blot analyses after Pstl digestion. The distance between the Pstl sites in the wild type (top) and mutant (bottom) locus is shown. (b) Southern blot analyses of genomic DNA from wild type and targeted ES cells after Pstl digestion with a 3'probe. The wild type Pstl fragment has a size of $4.9 \mathrm{~kb}$, whereas the mutant Pstl fragment is $3.0 \mathrm{~kb}$.

Aldrich F3777, dilution 1:3000) or biotinylated mouse monoclonal R4A specific for smoothelin $^{23}(1 \mu \mathrm{g} / \mu \mathrm{l}$; dilution 1:40). For $\alpha$-SMA, a secondary antibody coupled to horseradish peroxidase (1:300) was applied followed by staining with the AEC substrate kit according to the manufacturer's protocol (Vector Laboratories, Burlingame, CA). For detection of smoothelin, the ABC-peroxidase kit (Vector Laboratories) was used, followed by diaminobenzidine tetrahydrochloride-staining.

\section{Histology}

Tissues from neonatal and adult $\mathrm{SMTN}^{+/+}, \mathrm{SMTN}^{+/}$, and $\mathrm{SMTN}^{-1}$ mice were fixed overnight in $3.7 \%$ formaldehyde in phosphate buffered saline (PBS), embedded in paraffin, sectioned, and stained with haematoxylin and eosin.

\section{Food transit time test}

For determination of the intestinal function, a food transit time test was employed as previously described ${ }^{525} .100 \mu 16 \%$ carmine in PBS ( $\left.\mathrm{pH} 7.0\right)$ was injected into the stomachs of six $\mathrm{SMTN}^{+/+}, \mathrm{SMTN}^{+/}$, and $\mathrm{SMTN}^{-/}$mice, and faeces were monitored for the first appearance of red dye. 


\section{Cardiovascular physiological assessment}

Mean arterial pressure (MAP) and heart rate (HR) of five female $\mathrm{SMTN}^{+/ t}$ and $\mathrm{SMTN}^{-/}$ littermates of approximately 6 weeks old were recorded for 10 min under 1-2\% isofluorane anesthesia. A pressure transducing catheter was introduced into one of the carotid arteries, and MAP was measured as previously described ${ }^{526}$.

\section{Vascular contractility}

The thoracic aorta $(n=5)$ and femoral artery $(n=6)$ of 5-10 weeks old mice were dissected from surrounding tissue for measurements of arterial reactivity. Isolated arteries were mounted as ring segments between an isometric force transducer (Kistler Morce DSC 6, Seattle, WA) and a displacement device in a myograph (model $610 \mathrm{M}$, J.P. Trading, Aarhus, Denmark) employing two stainless steel wires (diameter $40 \mu \mathrm{m})^{527}$. During mounting and experimentation, the myograph organ bath ( $5 \mathrm{ml}$ volume) was filled with Krebs-Ringer bicarbonate solution maintained at $37^{\circ} \mathrm{C}$ and aerated with $95 \% \mathrm{O}_{2}-5 \% \mathrm{CO}_{2}$. Reactivity to several agonists $\left(\mathrm{K}^{+}(40 \mathrm{mM})\right.$, U-46619 (thromboxane A2 mimetic) (0.1-100 nM), phenylephrine (PHE) (0.01-30 $\mu \mathrm{M})$, all obtained from Sigma-Aldrich) was examined. Between experiments, the arterial preparations were allowed to recover for at least $15 \mathrm{~min}$. The experiments were terminated by fixing the vessels in phosphate-buffered $(\mathrm{pH} \mathrm{7.4)}$ formaldehyde (4\%) for $30 \mathrm{~min}$ at $37^{\circ} \mathrm{C}$.

\section{Morphometry of arteries}

Fixed vessels were transferred to $70 \%$ ethanol and embedded in paraffin. Radius and medial cross-sectional area (CSA) were determined using a Zeiss Axioscope (Zeiss, Germany), a standard CCD camera (Stemmer, Germany), and commercially available software (JAVA 1.21, Jandel Scientific, Corte Madera, CA) after staining thin sections with van Gieson's solution. CSA was defined as the area between the internal elastic lamina and the external elastic lamina bordering the adventitia ${ }^{528}$.

\section{Statistical analysis}

Statistical significances were calculated by repeated measures one-way ANOVA followed by Bonferroni's multiple comparison test or two-tailed paired Student's ttests using Graphpad Prism software (version 4.0). Groups were considered significantly different at $P<0.05$.

\section{Results}

The SMTN gene was targeted by substitution of exons 18 through 20 by a neomycin-resistance cassette in reversed orientation (figure 7.1a). Insertion of the cassette resulted in smaller transcripts which corresponded to splice variants with exons 16 or 17 spliced to exon 21, resulting in an intact reading frame (figure 7.2a). 
a

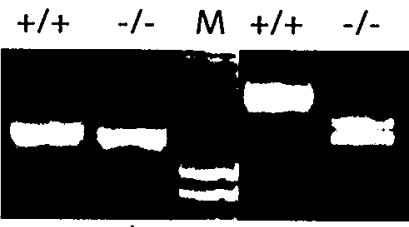

1F-1R

$2 \mathrm{~F}-2 \mathrm{R}$

b
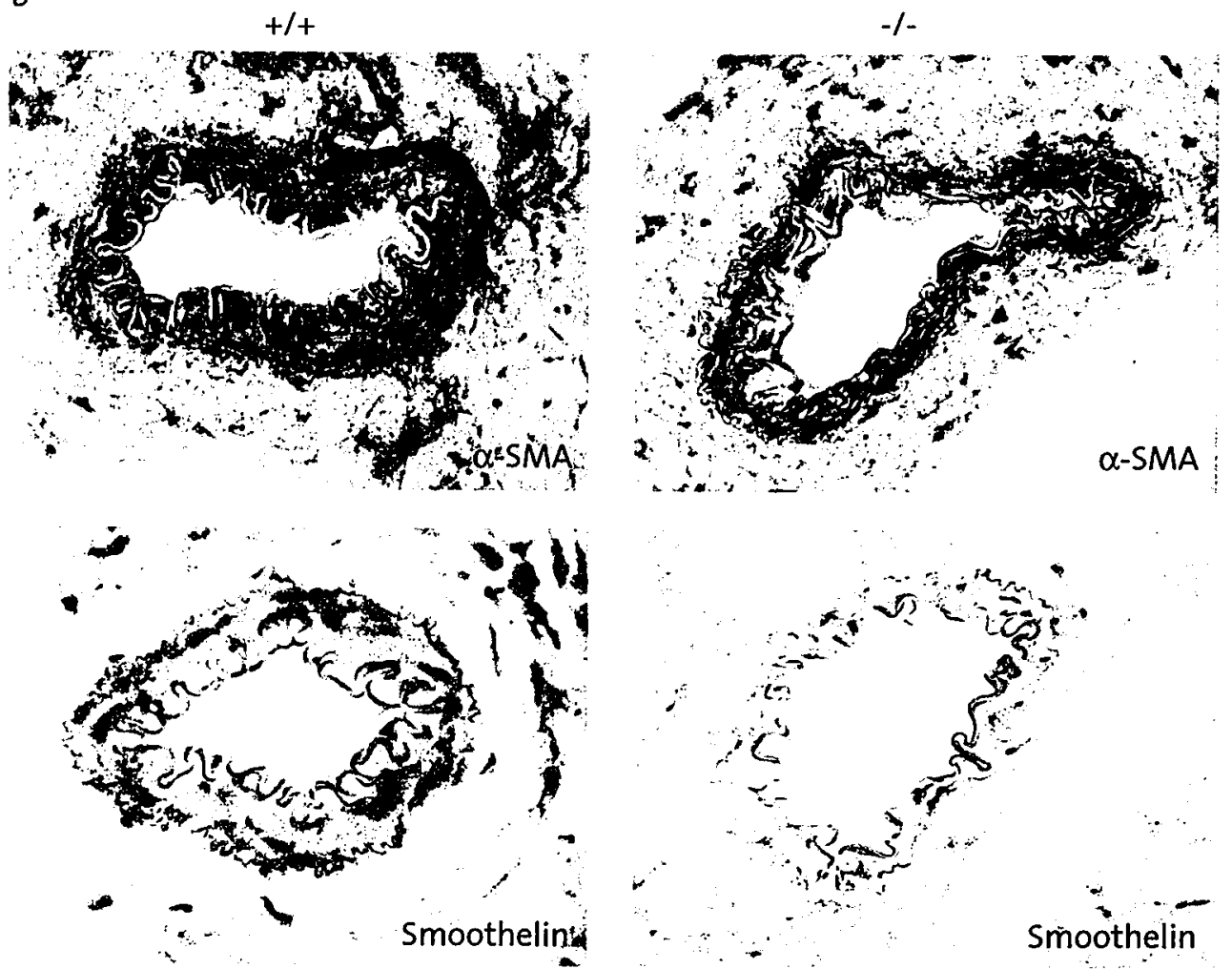

Figure 7.2 Expression of smoothelin mRNA and smoothelin protein in SMTN"/+ and SMTN"/ mice. (a) Analysis of smoothelin transcripts by RT-PCR. RNA isolated from the colon of SMTN"/+ and SMTN" mice was analyzed using different pairs of primers as indicated in figure 7.1a. In mRNA from the targeted allele, either exon 16 or exon 17 is spliced to exon 21. (b) Immunohistochemical stainings of crosssectioned femoral arteries of SMTN ${ }^{+/ 4}$ and SMTN" mice with anti- $\alpha$-SMA or anti-smoothelin antibodies. SMTN $\%$ mice do not express detectable levels of smoothelin despite normal $\alpha$-SMA staining. SMTN $\%$ femoral arteries have a significantly reduced wall thickness. See also color picture on back turnover.

However, no protein was detected in smooth muscle tissues using an antibody against an epitope upstream of the deletion (figure 7.2b). SMTN $/ 1$ mice were generated from $\mathrm{SMTN}^{+/}$mice. Pups were delivered at an approximately Mendelian ratio with no sexual bias (distribution: $\mathrm{SMTN}^{+/+} 30 \%, \mathrm{SMTN}^{+/} 46 \%, \mathrm{SMTN}^{-/} 24 \%$; $n=415$ ), showing that loss of smoothelin is not embryonically lethal. However, 
about $30 \%$ of the $\mathrm{SMTN}^{-1-}$ mice died within the first week (figure 7.3 ). During the next two weeks, progressively more mice died, and only $50 \%$ of the $\mathrm{SMTN}^{\circ}$ mice reached weaning age (21 days). SMTN ${ }^{-1-}$ mice had a normal birth weight (data not shown), but became smaller and lighter than their $\mathrm{SMTN}^{+/ \cdot}$ and $\mathrm{SMTN}^{+/+}$ littermates. SMTN ${ }^{-1}$ mice surviving for several weeks reached a stable, but approximately $20 \%$ lower body weight than $\mathrm{SMTN}^{+/-}$and $\mathrm{SMTN}^{+/+}$mice. No offspring could be generated from SMTN ${ }^{-1}$ males. SMTN ${ }^{+/ \cdot}$ mice were phenotypically normal and had a normal life span.

\section{Survival proportions}

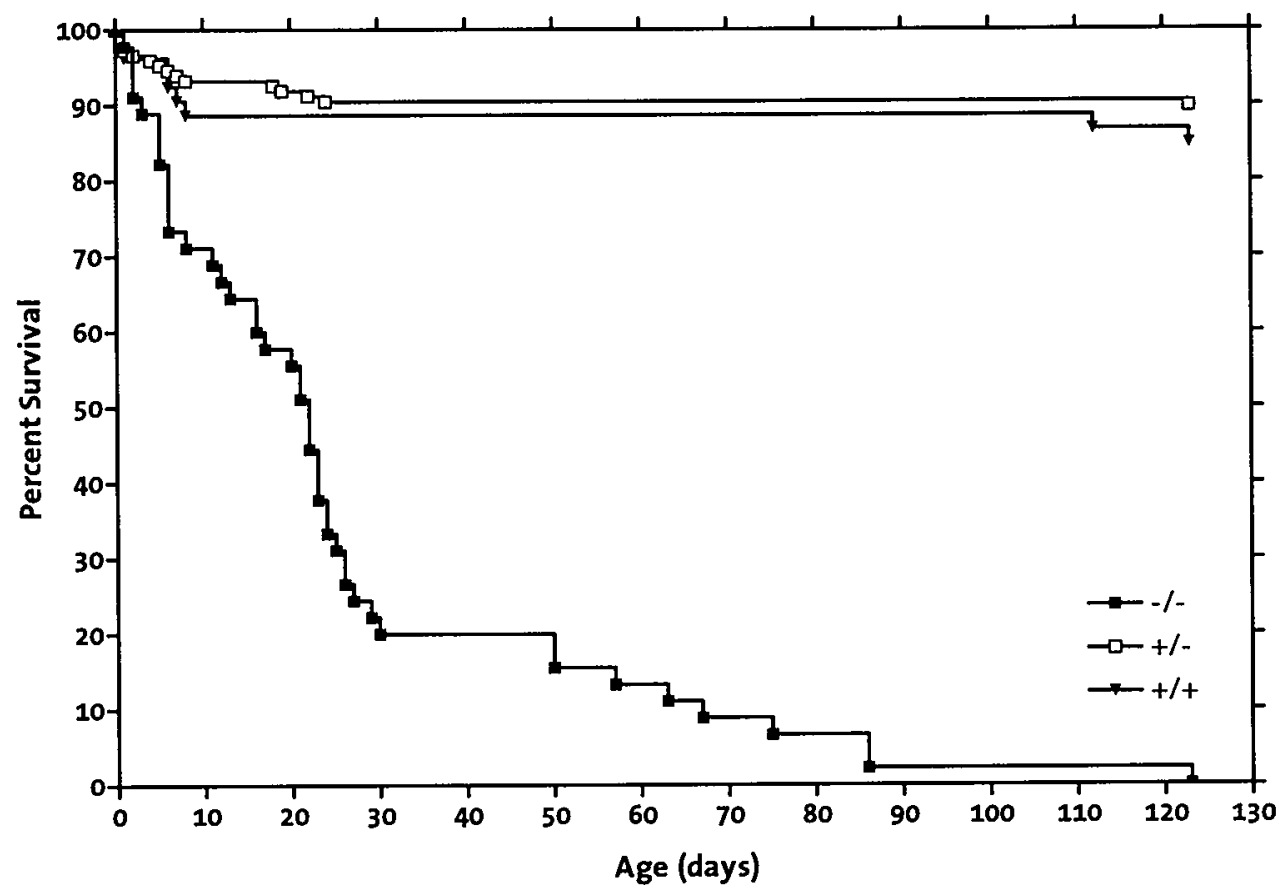

Figure 7.3 Survival curve of SMTN ${ }^{+/ 4}, \mathrm{SMTN}^{+/}$, and $\mathrm{SMTN}^{\prime /}$ mice. SMTN ${ }^{\prime /}$ mice are viable but die after birth. SMTN ${ }^{+1}$ mice have a normal life expectancy. $(n=263)$.

\section{Pathology}

All tissues of sacrificed mice $(n=29)$ were examined for pathological anomalies. Prominent smooth muscle tissue aberrations were observed in all $\mathrm{SMTN}^{-1-}$ mice, at all ages. Affected organs included the gastro-intestinal tract, the urinary tract, the biliary tract, the vascular system, and the reproductive organs (table 7.1). Other tissues were not affected. Macroscopically, we saw a greatly enlarged duodenum in $90 \%$ of the SMTN ${ }^{\prime}$ mice, with diverticula in the cecum, small intestine, and large intestine. The intestinal tract was extremely rigid and fragile. SMTN ${ }^{-1}$ mice showed less visceral and subcutaneous fat deposition. Enlargement of the gallbladder ( $30 \%$ of the $\mathrm{SMTN}^{-1}$ mice) and uni- or bilaterally enlarged kidneys (20\% of the $\mathrm{SMTN}^{-1}$ 
mice) were commonly observed anomalies. In addition, the blood vessels were relatively fragile. In female SMTN ${ }^{-1}$ mice, the uterus was reduced by $80 \%$, whereas in males the seminal vesicles were reduced by $70 \%$.

Table 7.1 Occurrence of smooth muscle pathologies in SMTN\% mice.

$\begin{array}{cc}\text { Pathology } & \text { Prevalence in SMTN } \\ \text { Intestinal diverticula } & 100 \% \\ \text { Underdeveloped reproductive organs } & 100 \% \\ \text { Reduced media thickness of muscular arteries } & 100 \% \\ \text { Enlarged and thin-walled duodenum } & 90 \% \\ \text { Hydronephrosis } & 43 \% \\ \text { Enlarged gall bladder or periportal inflammation } & 29 \%\end{array}$

Microscopically, we observed that the diverticula in the intestinal tract occurred mostly on the mesenteric side, where vessels and nerves enter the muscular wall (figure 7.4a). The diverticula were often filled with inspissated faeces. Inflammation of the diverticula and the mesentery was common, though an inflammatory perforation was rare. The intestinal villi in the affected areas were irregular and atrophic. In $29 \%$ of the livers, we observed periportal inflammation, and in some cases hepatocellular necrosis was seen (figure $7.4 \mathrm{~b} / \mathrm{c}$ ). Sometimes bile duct hyperplasia was seen. A large proportion of $5 \mathrm{MTN}^{-1}$ mice $(43 \%)$ displayed hydronephrosis. In severe cases ( $20 \%$ of the mice), this resulted in a very thin cortex and reduced renal tissue. Some of these mice showed extensive ureteral inflammation and hyperplasia of ureteral SMCs (figure 7.4d/e). The medial crosssectional area (CSA) of the femoral artery of $\mathrm{SMTN}^{\prime-}$ mice was significantly reduced as a result of SMC atrophy (figure 7.2a \& 7.5a). Though the radius of the femoral artery and both radius and CSA of the thoracic aorta of SMTN ${ }^{-1}$ mice were not significantly altered, they were reduced to some extent (figure 7.5). Some SMTN\% mice, aged around three months, displayed an inward growth of SMCs in the aorta at the iliac bifurcation, as well as neointimal growth in smaller blood vessels. This cell mass was poorly structured and contained inflammatory cells and epithelioid SMCs.

\section{Intestinal function}

The histological data indicated that smooth muscle function in SMTN" mice might be compromised. Therefore, we assessed their intestinal performance. The whole gut transit time in SMTN ${ }^{-1}$ mice was approximately doubled compared with $\mathrm{SMTN}^{+/+}$or SMTN ${ }^{+/}$littermates, despite a comparable length of the gastro-intestinal tract (figure 7.6). Preliminary results on the contractile activity of intestinal muscle strips of SMTN $\%$ mice indicate that they have irregular slow waves with small amplitudes. 
a
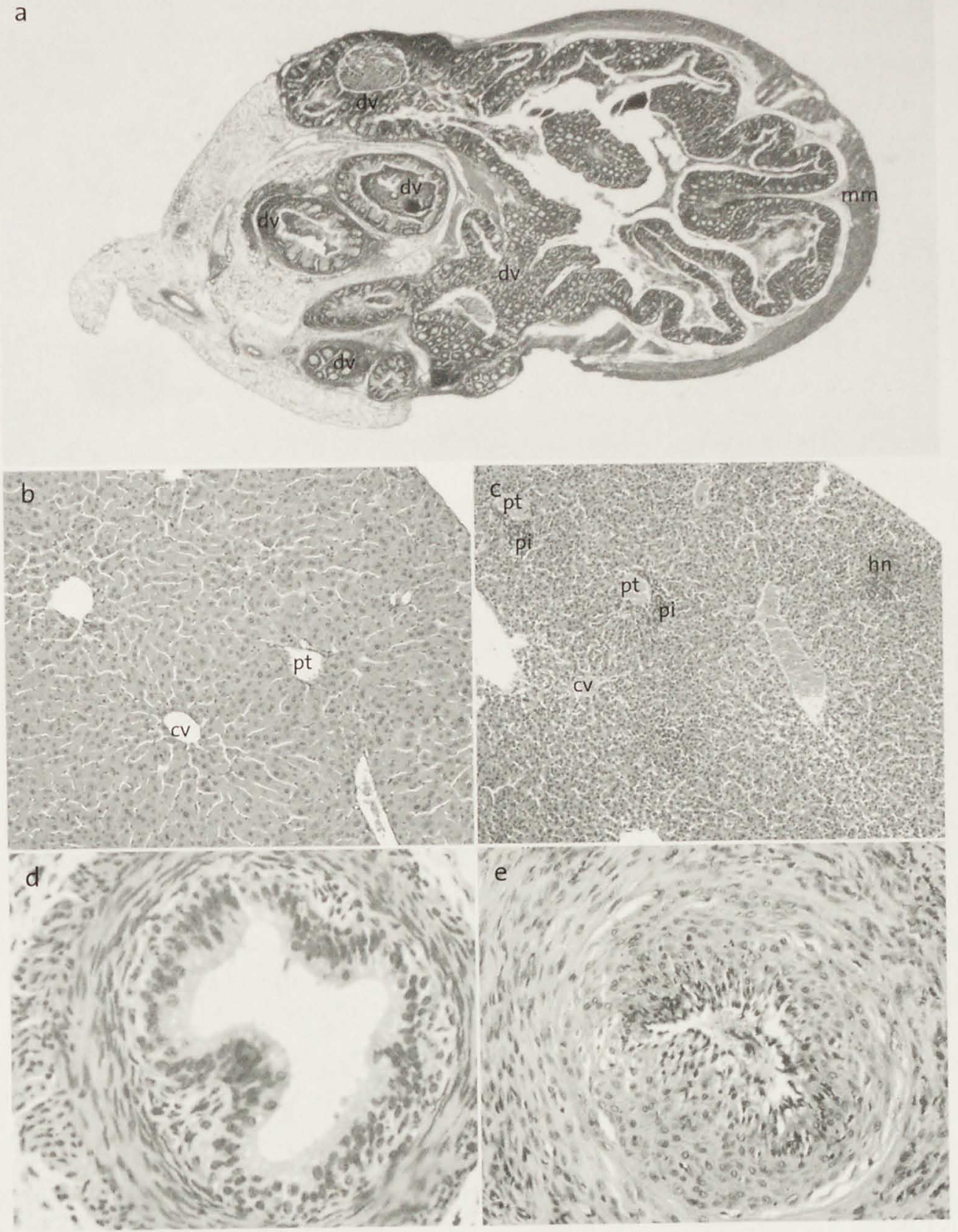

Figure 7.4. Histology of SMTN ${ }^{+/+}$and $\mathrm{SMTN}^{-1}$ mice. (a) Cross-section of a colon of a SMTN ${ }^{-/}$mouse showing typical severe diverticulosis (dv). Little or no muscularis mucosa $(\mathrm{mm})$ is present on the side where the diverticula are observed. (b) Liver of a SMTN ${ }^{+/+}$mouse showing no abnormalities (cv-central vein; pt-portal triad; magnification 10x). (c) Liver of a SMTN ${ }^{-1}$ mouse with periportal inflammation (pi) and hepatocellular necrosis (hn) (magnification 10x). (d) Ureter of a SMTN mouse without

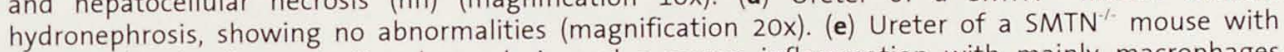
hydronephrosis, demonstrating hyperplasia and a severe inflammation with mainly macrophages (magnification 20x). See also color picture on back turnover. 

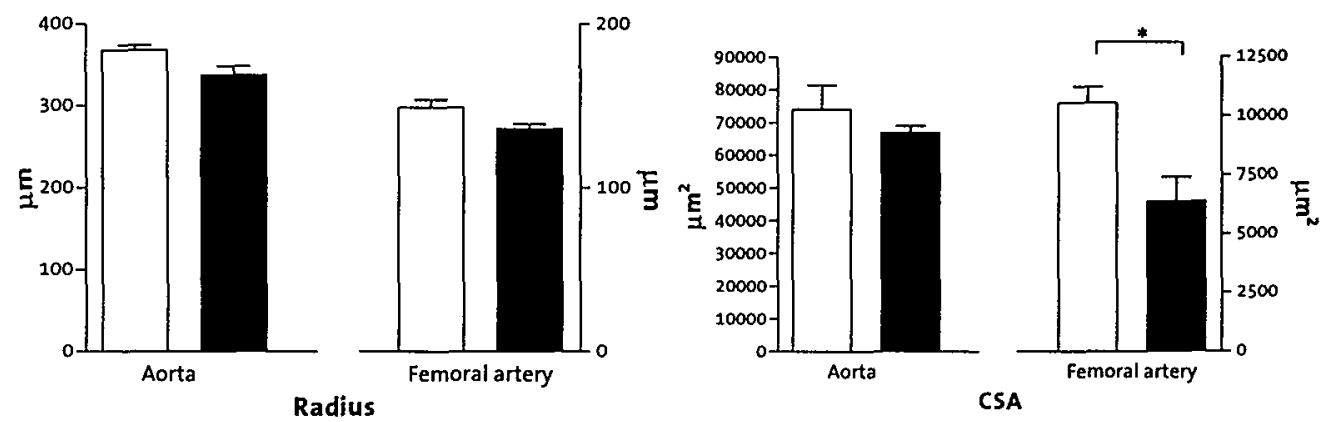

Figure 7.5 Radius and medial cross-sectional area (CSA) of the thoracic aorta and femoral artery of $S M T N^{+/ t}$ and SMTN"/ mice. SMTN ${ }^{+/ t}$ mice are represented by white bars and SMTN ${ }^{-1 /}$ mice by black bars. Values are expressed as mean \pm standard error of the mean (SEM). The femoral medial CSA is significantly diminished in SMTN $*$ mice. Statistically significant differences $(P<0.05)$ as determined by the paired Student's t-test are indicated by an asterisk.

\section{Vascular function}

In contrast to the intestinal function, most cardiovascular parameters did not differ significantly between SMTN ${ }^{/}$and SMTN ${ }^{+/+}$littermates, although MAP, HR, and the heart weight to body weight ratio tended to be higher in $\mathrm{SMTN}^{\prime \prime}$ mice (table 7.2).

Table 7.2 Comparison of cardiovascular parameters in SMTN ${ }^{+/ 4}$ and SMTN $\%$ mice.

No significant differences were detected apart from bodyweight (indicated by an asterisk).

$\begin{array}{ccc}\text { Parameter } & \text { SMTN }^{+/+} & \text {SMTN }^{\prime-} \\ \text { Age (days) } & 69 \pm 14 & 70 \pm 15 \\ \text { Bodyweight }(\mathrm{g}) & 21,4 \pm 2,0 & 17,4 \pm 1,3^{*} \\ \text { Heartweight }(\mathrm{mg}) & 124 \pm 12 & 113 \pm 10 \\ \text { Heart/Bodyweight ratio }\left(\%^{*} 100\right) & 58 \pm 4 & 68 \pm 9 \\ \text { Mean arterial pressure }(\mathrm{mmHg}) & 87 \pm 2 & 95 \pm 5 \\ \text { Heart rate (beats per minute) } & 468 \pm 23 & 517 \pm 12\end{array}$

Analysis of the contractile function of vascular smooth muscle of SMTN ${ }^{-/}$and $\mathrm{SMTN}^{+/+}$ mice by myograph experiments revealed fundamental differences. The contractile force generated by the femoral artery and the thoracic aorta of SMTN $\%$ mice in response to various vasoconstricting compounds was diminished (figure 7.7a-c). Whereas the difference in the aorta was only significant during PHE stimulation, the femoral artery of SMTN ${ }^{-1}$ mice was significantly less responsive to $\mathrm{K}^{+}, \mathrm{PHE}$, and U46619. This reduction persisted after correction for the reduced CSA of the femoral artery (figure 7.7d). Interestingly, the rate of relaxation (defined as the loss of tension after 1 min of relaxation induced by washout of U46619) was also significantly reduced in SMTN" mice, almost six-fold in the femoral artery (figure 7.7e). The reduction was less pronounced in the thoracic aorta, but even in this elastic artery, relaxation was two-fold reduced. 


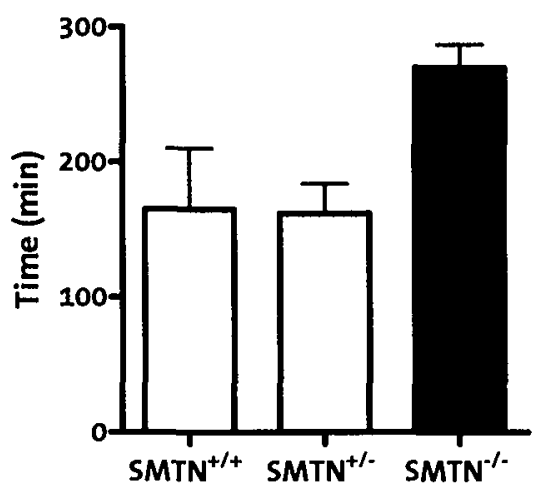

Figure 7.6 Whole gut transit time in $\mathrm{SMTN}^{+/ 4}, \mathrm{SMTN}^{+/}$, and SMTN $\%$ mice.

Transit time in $\mathrm{SMTN}^{\prime}$ mice was significantly longer than in $\mathrm{SMTN}^{+/+}$and $\mathrm{SMTN}^{+/-}$mice, suggesting diminished peristaltic activity $(P<0.05$ in a repeated measures ANOVA followed by Bonferroni's multiple comparison test).

\section{Discussion}

Smooth muscle cell contraction is dependent on a complex array of proteins, including SMC-specific actin and myosin isoforms, contractile filament-associated proteins, and cytoskeletal proteins. Investigations on the importance of SMCspecific contractile protein isoforms for smooth muscle function in knockout mice have shown that the characteristics of SMC contraction are altered in the absence of these proteins $5^{60,65,66.72}$. Yet, probably because of redundancy, SMC contractile function in most of these models is sufficient to maintain adequate tissue performance, and deficiency of proteins like $\alpha-S M A^{60}, S M-B$ myosin heavy chain ${ }^{65}$, $S M$-calponin ${ }^{72}$, and $S M 22 \alpha^{68}$ is compatible with life. Here we show that, unlike these other proteins, smoothelin deficiency in mice causes severe smooth muscle contractile dysfunction, which ultimately leads to premature death.

Although loss of smoothelins does not affect in utero survival, it leads to lethal pathologies in several smooth muscle tissues at an early age. Two main causes of death can be identified: 1) intestinal inflammation due to diverticulosis, leading to starvation or intestinal rupture, and 2) renal failure caused by severe hydronephrosis.

The presented evidence indicates that these phenotypes result from a loss of SMC contractile performance. Diminished contractility of the intestinal wall is known to lead to an increased intraluminal pressure, because of accumulation of food ${ }^{529-531}$. This provokes herniation of mucosa and submucosa through the mesenteric side of the bowel wall, where it is weaker since it is penetrated by paired mesenteric blood vessels ${ }^{532}$. The hydronephrosis in SMTN $^{-1}$ mice is likely due to diminished contractility of ureteral SMCs, which leads to accumulation of urine. In classical animal models ${ }^{533}$, blockage of urine excretion through ureteral obstruction causes epithelial damage, resulting in SMC proliferation. This SMC accumulation was also observed in some SMTN"- mice, and may lead to further accumulation of urine, aggravating the phenotype. 
a

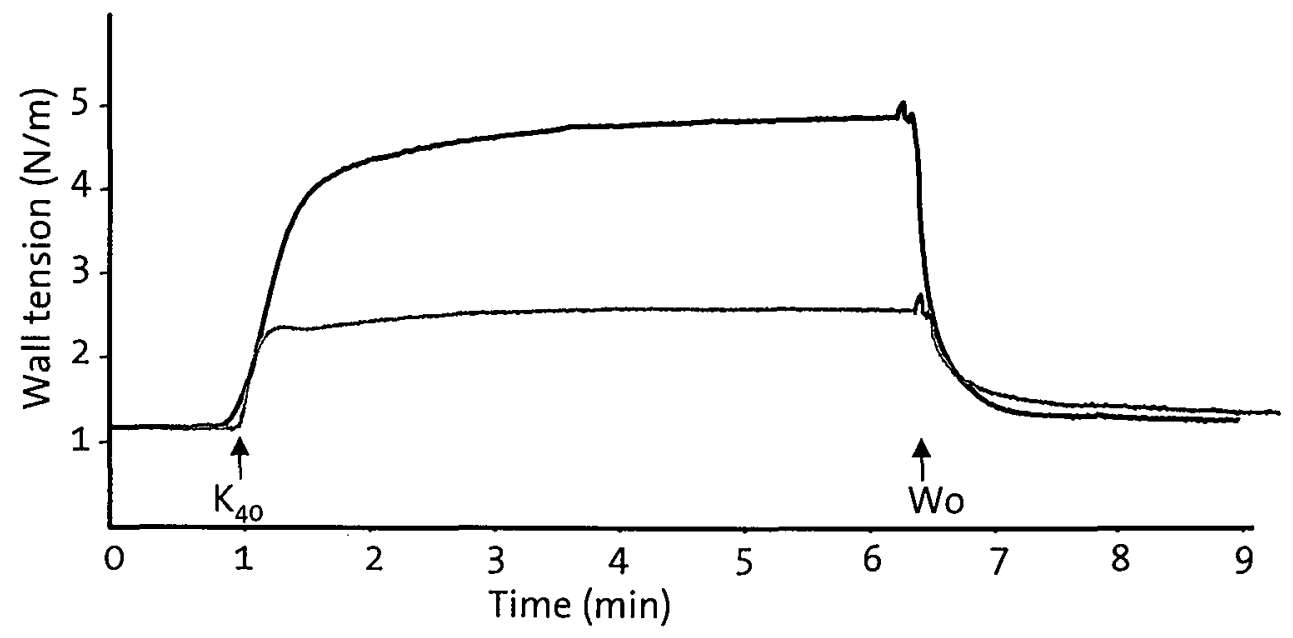

b
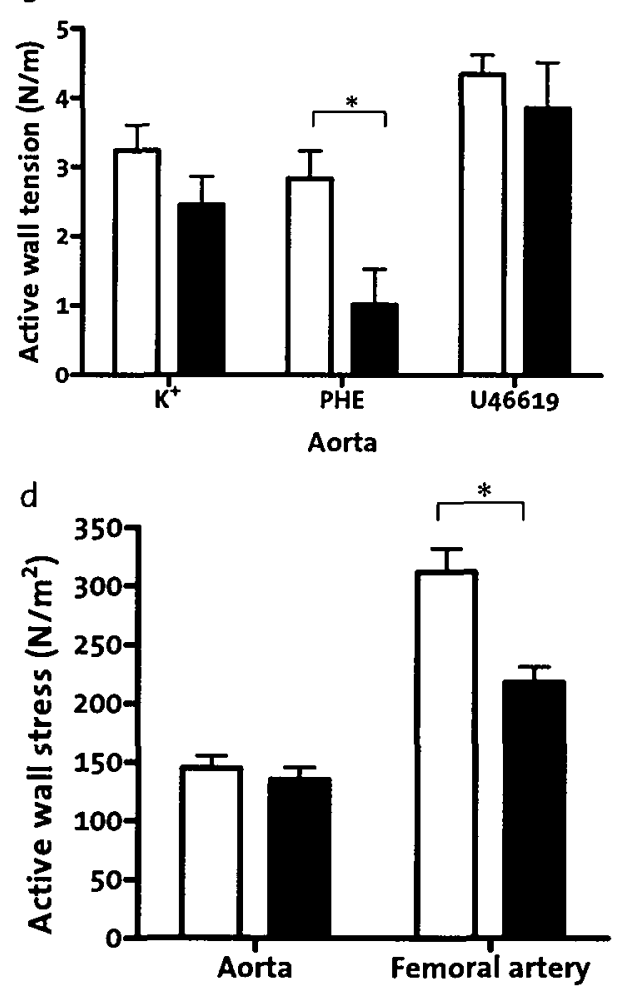

C

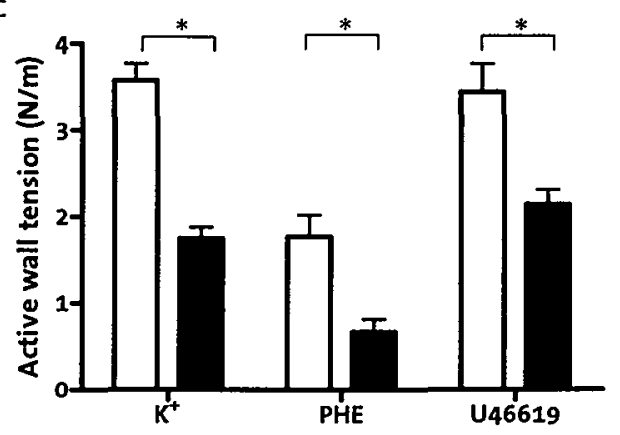

Femoral artery

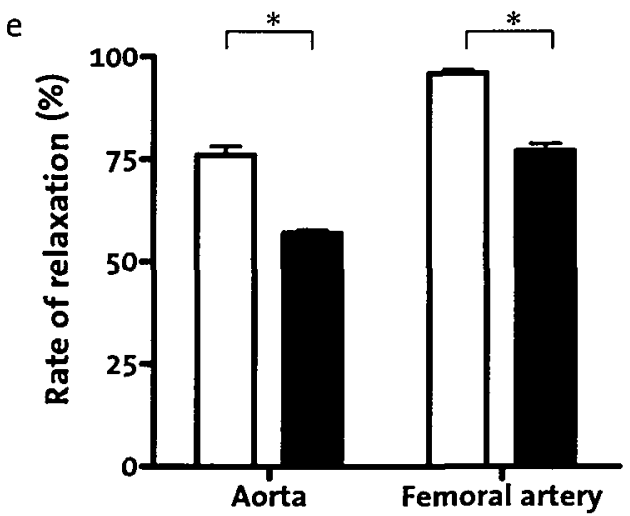

Figure 7.7 Contractile reactivity of $\mathrm{SMTN}^{+/+}$and $\mathrm{SMTN}^{-1-}$ thoracic aorta and femoral artery. (a) Typical tracings of isometric tension versus time of femoral arteries of $5 \mathrm{MTN}^{+/+}$(black line) and $\mathrm{SMTN}^{-1}$ mice (grey line) upon stimulation with $40 \mathrm{mM} \mathrm{K}$ (indicated by $\mathrm{K}_{40} ;$ Wo indicates washout of $\mathrm{K}^{+}$). Maximal contractile responses to $\mathrm{K}^{+}$, phenylephrine (PHE), and $\mathrm{U} 46619$ on thoracic aorta (b) and femoral artery (c) of $\mathrm{SMTN}^{+/+}$(white bars) and SMTN ${ }^{\prime /}$ mice (black bars). (d) Comparison of active wall stress in thoracic aorta and femoral artery of SMTN ${ }^{+/+}$(white bars) and SMTN ${ }^{\prime-}$ mice (black bars). Active wall stress is the active wall tension corrected for differences in CSA. (e) Loss of tension 1 minute after relaxation induced by washout of $U 46619$. Asterisks indicate significant differences between $\mathrm{SMTN}^{+/+}$and $\mathrm{SMTN}^{-1}$ mice. 
The periportal inflammation of the liver and gall bladder enlargement, which are the first symptoms of cholestasis in the mouse ${ }^{534}$, can also be explained by a loss of contractility, in this case of the bile duct. It is not clear whether the infertility of male SMTN $\%$ mice is due to a contractile dysfunction of the vasa deferentia, or a result of starvation caused by diminished intestinal performance, but the presence of mature sperm suggests the former.

Because of the transient expression of smoothelin in developing striated muscle cells at early embryonic stages ${ }^{82}$, it might be expected that SMTN ${ }^{-1}$ embryos would display problems in skeletal or heart muscle. However, the structure of these tissues appears normal, and birth weight does not differ between $\mathrm{SMTN}^{-1}, \mathrm{SMTN}^{+1}$, or $\mathrm{SMTN}^{+/+}$mice. In addition, the normal litter size and genotype distribution at birth shows that the absence of smoothelin during embryogenesis does not affect survival.

Up to now, the hypothesized function of smoothelins in SMC contraction has been based on circumstantial evidence, that is, their expression is restricted to contractile SMCs ${ }^{18,23,509}$, they colocalize with $\alpha$-SMA stress fibers ${ }^{23.83}$, they are upregulated in tissues that gain contractility ${ }^{483}$, and the expression of the two major smoothelin isoforms appears to be related to the type of SMC contraction ${ }^{82,511}$. The data obtained from our $\mathrm{SMTN}^{-1}$ mouse model provide direct evidence that smoothelins are not only involved in, but are also essential for normal smooth muscle contraction and relaxation.

This becomes apparent in the prolonged whole gut transit time in SMTN $\%$ mice, indicative of diminished intestinal peristalsis. Moreover, the response to vasoconstricting compounds is greatly reduced in $\mathrm{SMTN}^{-1}$ arteries. In addition, preliminary evidence on intestinal muscle strips of SMTN $\%$ mice indicates that slow wave contractile activity is diminished. Thus, the present results identify smoothelins as critical proteins for physiological smooth muscle contraction.

The relatively mild effects of smoothelin deficiency on the contractile properties of the aorta as compared to the femoral artery can be attributed to the fact that there is a much smaller number of SMCs in the media of this vessel. Moreover, only 5$10 \%$ of aortic SMCs express smoothelin, as opposed to $30-50 \%$ of the cells in the femoral artery media ${ }^{79}$. The fact that $\mathrm{SMTN}^{\%}$ mice do not have a significantly changed blood pressure or heart weight may reflect that the reduced smooth muscle contraction and relaxation in the muscular arteries of these mice are balanced. In this respect, it will be interesting to determine the ability of SMTN ${ }^{-1}$ mice to maintain blood pressure under stress conditions.

To investigate the pathways which might underlie the effects of smoothelin deficiency on smooth muscle contraction, we tested the response of SMTN ${ }^{-1}$ arteries to the vasoconstrictor compounds $\mathrm{K}^{+}, \mathrm{U} 46619$ (a stable thromboxane A2 mimetic), and $\mathrm{PHE}$, which induce smooth muscle contraction in different ways. 
Increasing extracellular $\mathrm{K}^{+}$concentration results in membrane depolarization and activation of voltage-gated $\mathrm{Ca}^{2+}$ channels, followed by influx of extracellular $\mathrm{Ca}^{2+535}$. Binding of U46619 to TP receptors stimulates SMC contraction by activating the phospholipase $\mathrm{C}$ pathway, which leads to intracellular $\mathrm{Ca}^{2+}$ release ${ }^{536}$. In addition, this compound increases the influx of extracellular $\mathrm{Ca}^{2+}$ through inhibition of $\mathrm{K}^{+}$ channels ${ }^{537}$, and activates Rho kinase and tyrosine kinases, which regulate the phosphorylation of myosin light chains and thereby contraction in various ways ${ }^{538.539}$. PHE interaction with $\alpha 1$-adrenoceptors causes both activation of phosholipase $\mathrm{C}$ and $\mathrm{Ca}^{2+}$ channels in the plasma membrane, with subsequent release of $\mathrm{Ca}^{2+}$ from intracellular stores and $\mathrm{Ca}^{2+}$ influx from the extracellular space ${ }^{527}$. Interestingly, although the degree of reduction was not uniform, reduced contractility in SMTN $\%$ arteries was observed regardless of the signal transduction pathways that were activated. Together with the fact that relaxation in SMTN ${ }^{-1}$ arteries was also diminished and the knowledge that smoothelins localize to the contractile apparatus itself, this non-selective reduction of contractility suggests that smoothelins are structurally involved in SMC contraction.

The mechanism by which smoothelins affect smooth muscle contraction needs more study. It has been shown that in other knockout mouse models of contractile SMC proteins, the observed phenomena may be explained by downregulation of other proteins involved in SMC contraction, by expression of different alternatively spliced contractile proteins, or by upregulation of related proteins, reflecting redundancy ${ }^{60,65,66,72}$. The lethal phenotype of our $5 \mathrm{MTN}^{-1}$ mice indicates that there is no protein that can replace the function of smoothelins. Database searches of the mouse genome corroborated this notion, since smoothelin homologs were not detected. It cannot be ruled out that changes in the levels or organization of other contractile proteins occur in SMTN ${ }^{-1}$ animals. Since we have knocked out both smoothelin isoforms, we cannot distinguish the effects of smoothelin-A deficiency from the effects of smoothelin-B deficiency, and it is possible that different isoforms are responsible for the different phenotypes encountered in SMTN ${ }^{-/}$mice. Yet, the observed reduction in contractility in both vascular and visceral smooth muscle indicates that both smoothelin isoforms are involved in contraction.

We conclude that smoothelin deficiency causes a decline in the contractile function of many smooth muscle tissues, giving rise to lethal abnormalities. Our findings indicate that alterations in smoothelin levels may play a critical role in diseases as diverse as diverticulosis, hydronephrosis, cholestasis, and infertility. Finally, in view of the incomplete understanding of the unique features of SMC contraction, the identification of smoothelins as proteins that are structurally involved in contraction offers new prospects for advancing our comprehension of this essential function of SMCs. 


\section{Acknowledgements}

We would like to thank Ben Janssen, Agnieszka Strzelecka, and Jaques Debets for the measurements of the cardiovascular parameters, and Gregorio Fazzi for the morphometric analysis. Technical assistance by our colleagues from the animal facility and by Darren Baker, Alexandra Oldenhof, Roel Brands, and Diane Fens is gratefully acknowledged. This work was supported by grants from Stichting De Gelderfonds, Stichting Simonsfonds, and the Netherlands Organization for Scientific Research (NWO). 



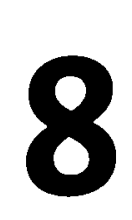

General discussion

Smoothelin: from a marker protein to a marked mouse 


\section{Smoothelin: a reliable marker for the contractile SMC phenotype}

Six years ago, smoothelin was first described as a cytoskeletal SMC-restricted protein that appeared to be associated with the contractile phenotype of these cells $^{79}$. As such, it was suggested that it might represent a good marker for contractile SMCs. Following up on this work, we have shown that with respect to other established contractile SMC markers, smoothelin is most responsive to modulation towards a synthetic phenotype in culture (chapter 2). Particularly the fact that smooth muscle myosin heavy chain (SM-MHC) isoform expression decreased to a much lesser extent relative to smoothelin showed that smoothelin provides a sharper definition of the contractile SMC phenotype than these proteins, which are considered the best markers of the contractile SMC phenotype. Since our study, many reports have confirmed the value of smoothelin as a marker protein to scrutinize the changes in SMC phenotype that are known to occur during culturing and diseases of smooth muscle tissues 3 ,18,19,22,509,540-544.

In agreement with its specificity for the differentiated contractile SMC phenotype, we have shown that smoothelin expression starts relatively late during development of the chicken embryo (chapter 3). During and after our study, fragmentary data on smoothelin expression during embryogenesis in rat, mouse, and human were published, which confirmed the status of smoothelin as the most distinctive marker of advanced contractile SMC differentiation ${ }^{81,541,545}$.

The use of smoothelin as a marker to discriminate between SMCs and related cell types like (myo)fibroblasts, and particularly between SMCs with an advanced contractile phenotype and a less contractile phenotype, is now well accepted. Future studies on diseases that are characterized by phenotypical changes of SMCS will benefit from the opportunity to detect those changes at an early stage by diminished smoothelin expression.

\section{Regulation of smoothelin expression}

\section{Physically separated promoters}

Whereas smoothelin-A is primarily expressed in visceral SMCs, smoothelin-B is predominant in vascular smooth muscle tissues. To explain this specificity of smoothelin isoform expression, studies aimed at elucidating the mechanism of generation of smoothelin-A and -B were initiated. Since several other SMC marker proteins are generated by alternative splicing mechanisms, it was expected that the smoothelin isoforms would arise in this way. Instead, we identified two promoters in the smoothelin gene that are $10 \mathrm{~kb}$ apart, and govern the expression of smoothelin-B or smoothelin-A, respectively.

The presence of two promoters in one gene that are separated by such a large genomic region containing exons is unusual. Often, two promoters regulating 
tissue-specific expression of two isoforms of a particular gene are closely spaced ${ }^{507}$. Consequently, the transcripts they generate differ only in their $5^{\prime}$ untranslated region, affecting their stability or translation efficiency, but not necessarily their function. For smoothelin, the two promoters clearly generate different proteins in different tissues, probably with different functions. At present, it is not clear why this mechanism of tissue-specific regulation of smoothelins has evolved, although regulation by alternative promoters appears to be a more direct way of regulation as compared to regulation by alternative splicing.

It is possible that the two promoters in the smoothelin gene interact with each other or even partly depend on each other. This interesting option can be tested by promoter reporter assays employing a vector in which the two regions upstream of the respective first exons of smoothelin-A and $-B$ are cloned simultaneously. These experiments can be extended by cloning an insulator sequence between the respective promoter fragments, which should block any effects of either promoter on the other's activity. Since patterns of promoter activity obtained by in vitro approaches are not always confirmed in vivo, we advocate confirming the activity of the two promoters in transgenic or knockin mice. Preliminary data from smoothelin-B-specific knockout mice that lack the genomic sequence encompassing exons 4-6 and a part of exon 7 indicate that these mice are still capable of generating smoothelin-A mRNA. Thus, this region does not seem to contain regulatory elements that are essential for smoothelin-A expression in vivo.

\section{Alternative splicing}

Even though alternative promoters are responsible for the generation of the two major smoothelin isoforms, alternative splicing is responsible for expression of smoothelin-A or -B variants that differ in their 3 ' region. The expression pattern of these alternatively spliced transcripts does not appear to be related to either tissue type, developmental stage, or SMC phenotype. Therefore, it will be difficult to identify the responsible regulators. The possible contribution of known SMC splicing regulators (e.g. polypyrimidine tract binding protein or proteins of the Bruno family) to splicing of the smoothelin gene can however be determined by UV-crosslinking experiments. In addition, identification of sequences known to mediate splicing of for example $\mathrm{h}$-caldesmon or meta-vinculin by homology searches may indicate which regions of the smoothelin gene are involved in the alternative splicing.

\section{Transcriptional regulation}

Detailed analysis of the smoothelin promoters revealed numerous cis-acting elements that are involved in regulation of other SMC-restricted genes. Of special interest were a transforming growth factor- $\beta$ (TGF- $\beta$ ) control element in the smoothelin-B promoter, a CAGA motif in the 5 ' untranslated region of smoothelin$B$, and two conserved CArG boxes in the smoothelin-A promoter. The CAGA motif, which has been conserved between the human and mouse smoothelin gene, was 
recently shown to regulate promoter activity of $S M 22 \alpha$ and $\alpha$-smooth muscle actin $(\alpha-S M A)^{298.546}$, and the TGF- $\beta$ control element in the human smoothelin- $B$ promoter has the same sequence as the ones in the $\alpha$-SMA and SM22 $\alpha$ promoters ${ }^{293}$. Surprisingly, this element, which is critical for TGF- $\beta$ inducibility of expression of $\alpha$ SMA and SM22 $\alpha^{186,293,327}$, has not been conserved between human and mouse in the smoothelin gene. In this light, it should be emphasized that smoothelin expression was not increased by TGF- $\beta_{1}$ in cultured pig coronary artery SMCs (chapter 2). Thus, this raises questions about the functionality of the TGF- $\beta$ control element and the CAGA motif. It cannot be ruled out that the TGF- $\beta_{1}$ dose employed in our study was not appropriate for induction of expression or that TGF- $\beta_{1}$ receptors were downregulated in our model. Alternatively, these motifs may not have been conserved in the pig smoothelin gene. It is noteworthy that TGF- $\beta_{1}$ treatment did increase smoothelin expression in canine prostatic SMCs at the same concentration ${ }^{540,547,548}$. This suggests that TGF- $\beta_{1}$ may have differential effects on smoothelin expression in different SMCs. To definitely establish the effects of these TGF- $\beta$-dependent elements on smoothelin promoter activity and its possible TGF- $\beta_{1}$ inducibility, reporter assays using smoothelin promoter fragments with mutated elements are required, preferably in transgenic or knockin mice.

Besides TGF- $\beta_{1}$, we have tested heparin for its effects on smoothelin expression in porcine SMCs. This molecule, which is known to induce contractile SMC differentiation, increased smoothelin levels three-fold. Though the effects of heparin on SMC marker gene expression are probably secondary to its inhibitory action on cell cycle progression and platelet-derived growth factor (PDGF) signaling ${ }^{549.550}$, it would be interesting to determine smoothelin promoter activity in the presence of heparin in the culture medium. Furthermore, it should be determined if blockage of the PDGF pathway or inhibition of proliferation leads to increased smoothelin levels.

A common theme in SMC-restricted genes is the occurrence of large 5' introns. Smoothelin is no exception to this rule. However, whereas the first intron of the $\alpha$ SMA, SM-MHC, and SM-calponin gene has been shown to be essential for expression 258.261 .510 , the first intron of the smoothelin gene actually attenuated promoter activity in cultured SMCs (unpublished observations). This does not preclude that this region may be important for smoothelin expression in vivo. The lack of promoter induction by addition of the first smoothelin intron is probably related to the absence of CArG boxes in this region, since these cis-elements were essential for the expression of the other genes.

Interestingly, the studies described in chapter 5 revealed that while CArG elements are of critical importance for the in vitro and in vivo expression of virtually all SMCrestricted genes identified at present ${ }^{268}$, they are not the dominant cis-elements in the smoothelin-A promoter in vitro. Assessment of their possible importance for smoothelin-A transcription in vivo awaits the generation of transgenic mice carrying a reporter gene under the mutated smoothelin-A promoter. However, it is 
likely that additional cis-elements are important for full activation of smoothelin-A transcription. The nature of these other elements is unknown at present. Important candidates are the conserved E-boxes that are present in an upstream region which increased smoothelin-A promoter activity in SMCs. These E-boxes, which bind basic-Helix-Loop-Helix (bHLH) transcription factors, have been found to mediate activation of several SMC-specific promoters. Indeed, it was recently shown that smoothelin and other SMC marker genes were only expressed after downregulation of Id1, which is an inhibitor of bHLH transcription factors ${ }^{548}$. Thus, it would be interesting to investigate the effects of $\mathrm{Id} 1$ and mutation of the E-boxes on smoothelin promoter activity.

Future studies may also identify those factors and elements that keep smoothelin expression restricted to SMCs of the contractile phenotype. At present, the only known transcriptional regulators of contractile SMC genes that have been reported to be downregulated when SMC modulate towards a synthetic phenotype are Gax, myocardin, and GATA-639,306,311. Since we showed that myocardin is a minor regulator of smoothelin-A expression (chapter 5), only Gax and GATA-6 or a not yet identified factor remain as candidates to restrict smoothelin expression to contractile SMCs. Alternatively, factors that inhibit expression of SMC genes in nonSMC, like HFH-1 ${ }^{551}$, may also prevent expression of smoothelin in synthetic SMCs. To restrict the area to be tested for contribution to the transcriptional regulation of smoothelin, sequence comparisons can be helpful. The Vista (visualization tools for alignments) comparative genomic tools (at http://www-gsd.lbl.gov/vista/) can compare mouse and human sequences at a large scale. In these analyses, conserved regions indicate the presence of either coding or regulatory elements. Using this tool, the conserved CArG boxes in the smoothelin-A promoter are readily identified (figure 8.1, arrow), in addition to other conserved regions with putative regulatory elements ${ }^{552,553}$.

The fact that the smoothelin gene contains two functional promoters that regulate expression in a rather strict-tissue specific manner presents many opportunities for the study of SMC differentiation. It shows that distinct regulatory pathways exist in visceral and vascular SMCs, even for the same gene. Moreover, the clear association between smoothelin expression and the contractile phenotype of SMCs indicates that the smoothelin promoters contain cis-elements that interact with transcription factors that are able to induce or maintain this phenotype. In addition, because a switch from the contractile to the synthetic phenotype is clearly involved in vascular disease, elucidation of the molecular regulation of smoothelin expression may increase our understanding of mechanisms responsible for this transition, and consequently may be useful to intervene in pathological processes. 


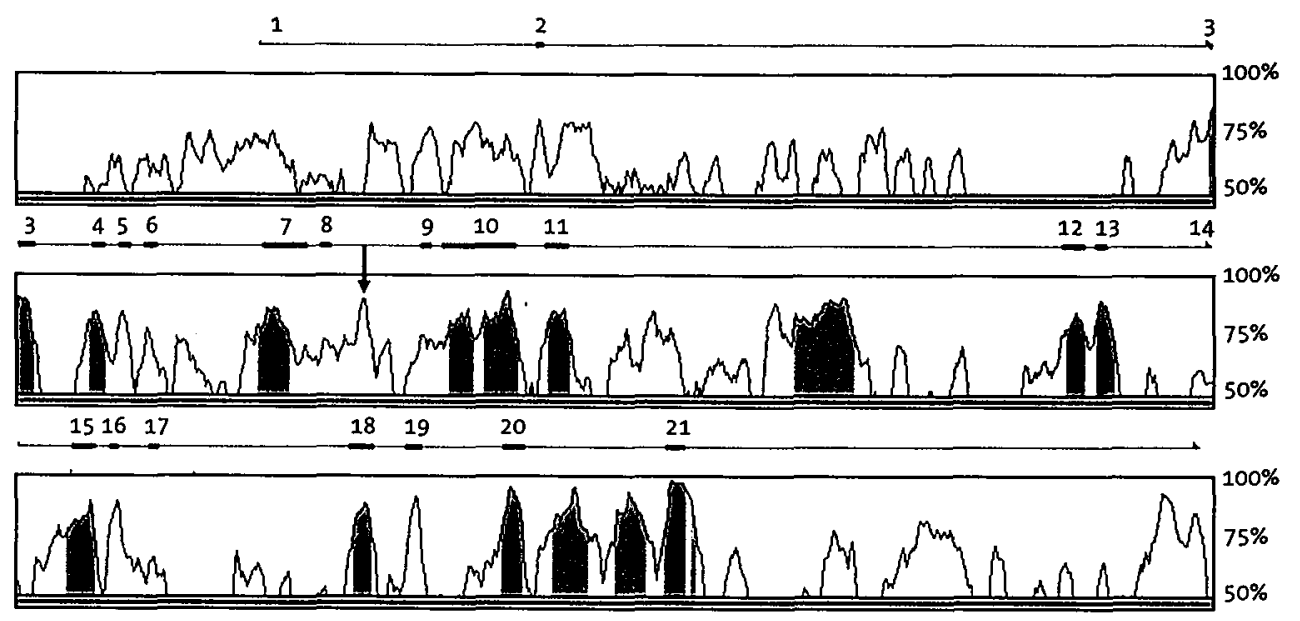

Figure 8.1 Vista alignment plot of the mouse and the human smoothelin gene region. On the $X$-axis, the base sequence of the human gene is plotted whereas the $Y$-axis represents the percent identity to the mouse gene. Exons are indicated on the upper lines above each panel. The smoothelin-A CArG box region is indicated by an arrow.

\section{Function of smoothelins}

Functional studies on smoothelins have proven difficult. Not only is it hard to study smoothelins in vitro because of the lack of expression in cultured cells, they are also rather insoluble proteins that precipitate under physiological ionic concentrations. Because of this, it became evident that we had to turn to an in vivo approach for the elucidation of the function of smoothelins. By generating smoothelin knockout mice and evaluation of the consequences of smoothelin deficiency at the tissue level, we have gained information about the importance of these proteins for SMC contractile function and differentiation in vivo.

\section{Consequences of smoothelin deficiency}

To enable targeting of the smoothelin gene in the mouse, it was cloned and sequenced (chapter 6). The critical role of smoothelins in smooth muscle contraction was subsequently revealed in smoothelin knockout mice as described in chapter 7. In these mice, diminished contractile performance of intestines, blood vessels, and ureters leads to problems with transport of food and urine, ultimately causing premature death. Although the results undoubtedly show that smoothelin deficiency affects smooth muscle function at the tissue level, it is not clear how the problems arise at the cellular level, i.e. which molecular mechanisms are targeted. Investigation of these mechanisms is critical to reveal if the observed phenomena are a primary effect caused by the lack of smoothelin, or secondary to the smoothelin deficiency. For example, since many proteins are involved in the regulation of SMC contraction, it is possible that changes in levels or isoforms of 
these proteins account for the observed changes in SMC contractile function in smoothelin null mice. However, the subcellular localization and domain structure (figure 8.2) of smoothelins indicate that they are involved in SMC contraction themselves.

Evidence for a direct role of smoothelins in SMC contraction: interaction with actin $\mathrm{We}^{23}$ and others ${ }^{224}$ have shown that smoothelins colocalize with $\alpha$-SMA, one of the main components of the SMC contractile machinery (chapter 2). Recently, immunoprecipitation and overlay experiments showed that both smoothelin isoforms interact directly with filamentous but not globular actin ${ }^{504.524}$. This interaction is at least in part mediated by smoothelin's 110 amino acid calponin homology $(\mathrm{CH})$ domain, a well known actin-binding domain ${ }^{554}$ (figure 8.2). Our group showed that this domain was capable of association with actin filaments, although more upstream sequences were required for strengthening of the binding ${ }^{504}$. Another study ${ }^{524}$ indicated that these upstream sequences were essential for full strength actin-binding of smoothelins and suggested that the $\mathrm{CH}$ domain only plays a supporting role in actin-binding, like it does in utrophin ${ }^{555}$. The actin-binding domain of utrophin and other spectrin superfamily proteins contains two $\mathrm{CH}$ domains in tandem repeat, which have a different affinity for actin ${ }^{554}$. Since smoothelin's CH domain is similar to the lower affinity $\mathrm{CH}$ domain, this may explain why additional domains are required for strong actin-binding. As smoothelins may contain slightly different $\mathrm{CH}$ domains (chapter 4), it would be of interest to determine whether their affinity for actin differs. Recently, it has been shown that the smoothelin-B specific part of the protein by itself localizes to actin filaments ${ }^{524}$. This may indicate that this part of the protein has actin-binding domains in addition to the actin-binding domains that smoothelin-B shares with smoothelin-A.

Regardless of the mechanism of binding, the fact that smoothelins bind actin filaments presents several possible functions of these proteins. They may regulate polymerization of actin, contribute to the stabilization of the filaments, determine their organization, or mediate interaction of other proteins with actin ${ }^{556}$. Because smoothelins only bind filamentous actin, a function in promoting actin filament assembly is less likely. A stabilization function of smoothelins cannot be excluded, but is not supported by any experimental data. In view of the reduced smooth muscle contractility in $\mathrm{SMTN}^{--}$mice (chapter 7 ) and the fact that reorganization of contractile filaments is a prerequisite for maximal SMC contraction, a function of smoothelins in organizing actin filaments cannot be ruled out. For a protein to act as a crosslinking protein that determines actin filament organization, it must contain two or more domains that interact with actin filaments, a criterion that appears to be met by smoothelin-B. It is known that a crosslinking protein can organize actin filaments into either bundles or meshed networks, depending on the number, affinity, spacing, and orientation of its filament-binding domains ${ }^{557}$. 
Therefore, in order to establish this possible function, these parameters should be determined for the different smoothelin isoforms. The fact that stress fibers containing tightly packed bundles of actin filaments can be formed in smoothelin deficient SMCs in vitro argues against their requirement for actin filament bundling. This does not exclude that smoothelins may support the formation of actin bundles. In fact, since actin bundles generate increased contractile force compared to uncoupled filaments and smoothelin deficient smooth muscle tissues have reduced contractile force, a role of smoothelins in bundling actin filaments is conceivable.

Evidence for a direct role of smoothelins in SMC contraction: homology to troponin T $S M C$ contraction is regulated in other ways than skeletal muscle contraction, although similar mechanisms have been shown to operate. For example, the SMC contractile machinery contains tropomyosin, an important protein that regulates $\mathrm{Ca}^{2+}$-dependent contraction in skeletal muscle by coordinating cooperative contraction by actomyosin cross-bridges. For its function in skeletal muscle, tropomyosin requires the troponin proteins, which are not expressed in SMCs. Instead, tropomyosin has been shown to interact with h-caldesmon and SMcalponin in SMCs, which partly take over the role of troponins. Interestingly, smoothelins contain a 37 amino acid sequence that is similar (35\% identical, $27 \%$ conserved residues) to the tail domain of troponin $T$ (figure 8.2). Since this domain is not only required for troponin $T$ interaction with tropomyosin but also involved in activation of actomyosin ATPase ${ }^{360}$, it would be of great interest to investigate a possible association of smoothelins with tropomyosin as well as their effect on myosin ATPase activity. In addition, the potential interaction of smoothelins with $\mathrm{h}$ caldesmon or SM-calponin should be investigated. Additional binding partners of smoothelins may be identified by yeast two hybrid analysis or similar systems.

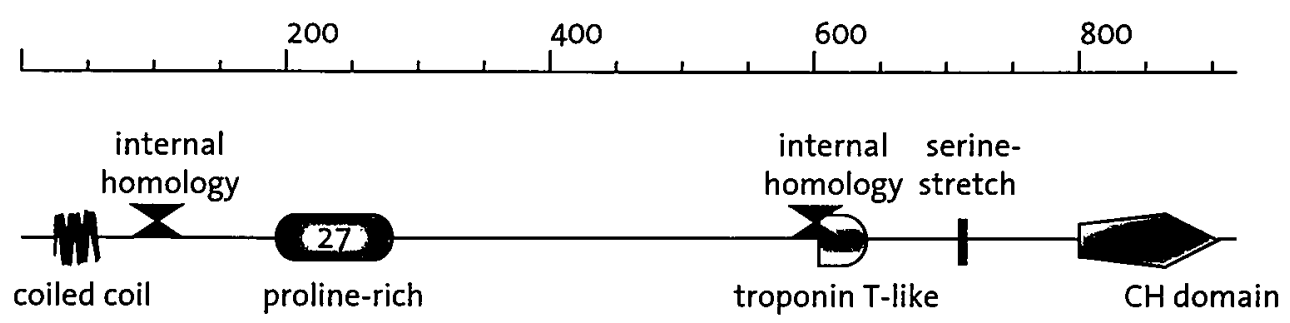

Figure 8.2 Schematic representation of the domain structure of smoothelin-B2. Apart from the $\mathrm{CH}$ domain and troponin T-like domain described in the text, $\mathrm{N}$-terminal amino acids that can adopt a coiled coil are indicated (calculated by the Simple Modular Architecture Research Tool-SMART at http://smart.embl-heidelberg.de/) ${ }^{2}$. A proline-rich region (27/88 amino acids) and a serine-stretch of 5 consecutive residues are depicted. Smoothelin-B variants contain a 37 amino acid region which is similar to a region downstream that is present in both smoothelin-A and smoothelin-B isoforms and overlaps with the troponin T-like domain (indicated by 'internal homology'). 
If the smoothelins are part of a tropomyosin-troponin-like system in SMCs, both the diminished contractile performance and reduced relaxation potential of vascular smooth muscle of smoothelin-deficient mice can be explained. Without a functional troponin-tropomyosin system, cooperativity of SMC contraction is compromised and myosin ATPase activity reduced. Consequently, contraction will still be possible in smoothelin deficient SMCs, but the lack of cooperativity causes a disorganized way of contraction. Contractile force will be diminished if not all cross-bridges of one contractile unit attach simultaneously after activation of contraction. Relaxation will be hampered because some cross-bridges of the contractile unit attach too late. Moreover, reduced myosin ATPase activity causes the crossbridges to remain attached to actin for a longer time, also hampering relaxation. Thus, both phenomena observed in the smoothelin knockouts can be explained by this model of uncoordinated contraction. Clearly, this mechanism is highly hypothetical at the moment, but should be investigated because it explains several aspects of the contractile dysfunction of smoothelin deficient SMCs.

\section{Different functional properties of the major smoothelin isoforms}

The smoothelin knockout mice described in chapter 7 do not express either major smoothelin isoform. Thus, we cannot ascribe any of the observed pathologies or physiological problems to the absence of one specific isoform neither can we distinguish between the possible function of smoothelin- $A$ and smoothelin-B by using these mice. However, the accumulated data suggest that expression of specific smoothelin isoforms may relate to the mode of contraction of smooth muscle tissues. More specifically, the expression pattern of smoothelins in chicken embryos of different stages indicates that smoothelin-A expression is predominant in phasic SMCs, whereas smoothelin-B is mainly found in tonic SMCs (chapter 3 ). It remains to be established whether expression of specific smoothelin isoforms and the nature of SMC contraction are causally related. The advent of smoothelin-B specific knockout mice will be helpful to answer this question. Preliminary data indicate that smoothelin-B deficiency has no effect on survival and does not lead to reduced growth. Therefore, future studies using these smoothelin-B knockout mice hold great promise for the elucidation of the function of smoothelin-B. Alternatively, studies on full smoothelin knockouts employing viral vectors with either smoothelin CDNA to rescue part of the phenotype in ex vivo contractile reactivity experiments may shed light on the functional properties of the different isoforms.

Although it is likely that the three different splice-variants of both major smoothelins have different functions, there is no obvious relation between their expression pattern and functional aspects of SMC contraction. To be able to address this topic, a better quantification of the concentration of the diverse isoforms in various smooth muscle tissues, for example by real-time PCR techniques, is a prerequisite. Since the different $\mathrm{CH}$ domains produced by the splice variants may have differential affinities for actin (see above), their expression may 
also relate to different contractile properties of smooth muscle tissues, although there is no evidence to prove this suggestion.

Smoothelins and the contractile phenotype: cause or effect?

A very intriguing finding in blood vessels and ureters of some knockout mice was that the SMCs in these tissues appeared to have a synthetic phenotype and had proliferated extensively, causing obstructions. We cannot prove whether these effects are primary, (i.e. directly caused by smoothelin deficiency), or secondary (i.e. caused by disorganization or irritation of the smooth muscle tissue because of reduced contractile performance). Nevertheless, this observation suggests that smoothelins keep SMCs in a contractile phenotype, inhibiting proliferation, or that full contractile potential of a SMC is necessary for maintenance of the contractile phenotype. The early disappearance of smoothelins in cultured SMCs that are modulating towards the synthetic type also indicates that its expression is not compatible with the process of phenotypic modulation. Indeed, the rapidity of the shutdown of smoothelin expression, both at the mRNA ${ }^{23}$ and the protein ${ }^{22}$ level, suggests that the reduction is an active process. It is possible that smoothelin functions as a brake on phenotypic modulation. Since smoothelin is a structural component of the cell, it is imaginable that it hampers the shape changes that are necessary for cell migration or cell division, both of which are associated with the synthetic phenotype.

In this respect, it is noteworthy that we have observed that the generation of cell lines stably expressing smoothelin is impossible because these cells stop proliferating ${ }^{504}$ (and unpublished observations). The inverse relation between Ki67, a marker of cell proliferation, and smoothelin expression in models of vascular injury also supports the hypothesis that smoothelin expression needs to be shut down in proliferating cells ${ }^{19.509}$. Moreover, it has been shown that the transcription factor p53 directly upregulates smoothelin expression in the $\mathrm{H} 1299$ cell line ${ }^{558}$. p53's main function is to halt cell cycle progression after DNA damage, making sure that DNA replication is accurate. It may be that smoothelins are involved in this process. Collectively, these observations provide an indication that smoothelin expression may be more causally related to SMC phenotype, i.e. that its presence halts synthetic phenotype-related SMC proliferation. Clearly, this potential function of smoothelins needs much more study. First of all, it should be confirmed that the accumulated SMCs in the knockouts indeed have a synthetic phenotype, for example by determining marker protein expression. Subsequently, newborn smoothelin knockout mice that are less likely to have acquired secondary pathologies should be screened for the presence of synthetic SMCs. These studies may be complemented by experiments on the effect of proliferation inhibitors on smoothelin expression in vitro, to discriminate between cause and effect. If these studies reveal that smoothelins do keep SMCs in the contractile phenotype, then it would be interesting to identify the domains responsible. A first approach would be to transfect different domains into cells and monitor proliferation. 
In conclusion, the studies on the smoothelins described in this thesis have revealed that these proteins differ from other SMC contractile marker genes in many ways. Their restricted tissue- and phenotype-specific expression, the regulation of this expression pattern by two physically distinct promoters, and the dramatic effects of their deficiency on SMC function makes them a captivating addition to the SMC proteome. 
Summary 
This thesis starts off with a chapter describing the hallmarks of smooth muscle cells (SMCs). Most hollow organs are lined by SMCs, whose primary function is to allow tissues to contract and relax. A second important function of SMCs consists of the synthesis of extracellular matrix (ECM) components, which contributes to the structural integrity of smooth muscle tissues. To be able to perform both contractile and synthetic functions, SMCs can adopt different phenotypes. Contractile and synthetic phenotypes are distinguished. Under certain conditions, SMCs of one phenotype can shift to the other phenotype, a process called 'phenotypic modulation'. This occurs for example after injury of smooth muscle tissues or during SMC culturing. The phenotype of a SMC is however not only dependent on the local conditions. The wide variety of phenotypes that is observed in vivo is also related to the diverse origins of SMCs, which include the mesoderm, the neural crest, and the pro-epicardial organ.

Phenotypes and differentiation stages of SMCs are characterized by 1) morphological features, 2) specific proliferation and migration properties, and 3) the expression level of so-called SMC marker proteins. Most of these marker proteins, which include $\alpha$-smooth muscle actin ( $\alpha$-SMA), smooth muscle myosin heavy chain isoforms (SM-MHC), SM22 $\alpha$, and smoothelins, are associated with the contractile phenotype.

The molecular regulation of the different SMC phenotypes and of SMC differentiation is incompletely understood, although numerous factors that affect SMC phenotype have been described. For instance, platelet derived growth factor promotes early SMC differentiation and stimulates modulation of contractile SMCs towards the synthetic phenotype. Transforming growth factor- $\beta$ (TGF- $\beta$ ), retinoic acid, and heparin, in contrast, promote the contractile SMC phenotype. Physical factors like the organization of the ECM, interactions with other cells, pressure, and flow also influence SMC phenotype.

Several SMC lines and mouse models have been developed to study the regulation of SMC phenotype and SMC differentiation. These have revealed the importance of the transcription factor serum response factor (SRF), which, by binding to a sequence element called the CArG-box, and by association with different cofactors, regulates SMC phenotype and differentiation. The CArG box and additional important regulatory elements like the TGF- $\beta$ control element, the CAGA-box, and E-boxes are found in the promoters of virtually all genes that are specifically expressed in SMCs. Recently, it has also become clear that chromatin remodeling and alternative splicing are important regulatory mechanisms for SMC phenotype determination.

Both contractile and synthetic SMCs contain proteins that are dedicated to their contractile or synthetic functions. For contractile SMCs, this concerns the 
contractile proteins $\alpha$-SMA, SM-MHC isoforms, and contractile filament associated proteins like smooth muscle calponin, $S M 22 \alpha$, and smoothelins. The expression of different isoforms of these proteins allows for specific characteristics of SMC contraction, which is largely regulated by the degree of phosphorylation of the myosin light chains. This in turn depends on the activity of myosin light chain kinase and myosin light chain phosphatase, which are regulated in $\mathrm{Ca}^{2+}$ dependent and $\mathrm{Ca}^{2+}$ independent manners. The protein classes that are involved in the functions of the synthetic SMC include ECM molecules like collagens and elastin, and enzymes like matrix metalloproteinases and their inhibitors, which control ECM remodeling.

Investigations on the differentiation, regulation, and function of SMCS are largely based on the characterization of SMC marker proteins. One of these marker proteins, smoothelin, is the central topic of this thesis. At the beginning of the studies described in the thesis, two smoothelin isoforms had been reported: a 59 $\mathrm{kDa}$ A-isoform primarily expressed in visceral smooth muscle, and a vascular smooth muscle-enriched $110 \mathrm{kDa}$ B-isoform. Both isoforms belong to the class of structural proteins that contain a C-terminal sequence homologous to an actin binding motif. They are encoded by a single copy gene that appears to be exclusively active in contractile SMCs. To increase the usefulness of smoothelins for the study of the differentiation, regulation, and function of SMCs, studies were initiated to advance our understanding of their expression, regulation, and function.

The expression of smoothelin-B relative to other contractile SMC marker proteins was studied in a new SMC in vitro model employing porcine coronary artery SMCs (chapter 2). Smoothelin-B expression was much more sensitive to the phenotypic modulation of these SMCs than the other marker proteins. The reduction of its expression could be inhibited by heparin, but not by TGF- $\beta$ or retinoic acid. In contrast to other cultured SMCS, the porcine coronary artery SMCs maintained smoothelin expression for some passages at a level sufficient to study its subcellular localization. It was shown that smoothelin-B colocalizes with $\alpha$-SMA in the stress fibers, but not with intermediate filaments.

Subsequently, the expression pattern of smoothelins during chicken embryogenesis was investigated (chapter 3 ). Smoothelins were first expressed at stage 17 of development in the primitive heart and the myotome of the somites. These tissues generated a specific isoform, designated smoothelin- $C$, which was shown to be a variant of smoothelin-A. Interestingly, smoothelin-B expression was initially found in both vascular (from stage 20) and visceral (from stage 29) SMCs. Around hatching, visceral SMCs exchanged smoothelin-B for smoothelin-A, which is the regular visceral isoform. At hatching, smoothelin- $C$ expression in the heart and the somites was not detectable anymore. The observed expression pattern indicates that smoothelin variants have different functions in SMC contraction. 
Next, the regulation of smoothelin expression was investigated (chapter 4). Two physically separated promoters in the single smoothelin gene were shown to govern the expression of the two smoothelin isoforms. The smoothelin-B promoter is located $5^{\prime}$ of the first exon, but the smoothelin-A promoter is intragenic, upstream of exon 10. A more detailed analysis of the smoothelin promoters (chapter 5) revealed that the smoothelin-B promoter is stronger than the smoothelin-A promoter in promoter reporter assays. Smoothelin-A transcription was shown to be regulated in part by a sequence element called the CArG-box, which was able to bind SRF. Myocardin, a co-activator of SRF, increased smoothelinA expression in a CArG-dependent manner. Smoothelin-B promoter activity, in contrast, was not significantly increased by myocardin. Thus, smoothelin-A and -B are regulated in different ways, and are not completely dependent on CArG-boxes, unlike most other SMC marker genes. In addition to this dual promoter system, alternative splicing within the actin binding domain was demonstrated.

Since investigations on the function of smoothelins in vitro were hampered by their rapid decline during SMC culturing, an in vivo approach was required. To enable the generation of a smoothelin knockout mouse for this purpose, the mouse smoothelin gene first had to be cloned, mapped, and sequenced (chapter 6). Fluorescence in situ hybridization showed that the mouse smoothelin gene was located on chromosome 11, band A2-A3. It contains 21 exons, which are organized in the same way as the human smoothelin exons. Targeting of exons 18, 19, and 20 of the mouse smoothelin gene, which encode the actin binding domain, led to the generation of functional smoothelin knockout mice (chapter 7). These mice died at an early age from smooth muscle cell dysfunction, causing pathologies in various organs. The mice developed intestinal diverticula, hydronephrosis, and cholestasis, and were infertile. Analysis of vascular tissues of smoothelin knockout mice showed that wall thickness, contractility, and relaxation were diminished. Intestinal contractility also appeared to be reduced. It was concluded that smoothelins are critical for physiological smooth muscle functioning, and that they appear to be structurally involved in smooth muscle cell contraction.

In the final chapter of this thesis, several experimental approaches that might be undertaken to gain further insight into the regulation and function of smoothelins are put forward. These include the generation of transgenic reporter mice to study the regulation of smoothelin transcription in vivo, and analyses of the effects of $\mathrm{E}$ box mutations on smoothelin promoter activity. More detailed examinations of the knockout mouse models generated in this study with respect to SMC contractile behavior, SMC proliferation, and SMC marker protein expression will increase our understanding of the function of smoothelins, the regulation of SMC phenotype, and the working of the SMC contractile apparatus. 
Samenvatting 
Dit proefschrift begint met een hoofdstuk waarin de belangrijkste kenmerken van gladde spiercellen worden beschreven. De voornaamste functie van deze cellen bestaat eruit dat ze ervoor zorgen dat holle organen kunnen samentrekken en weer ontspannen. Gladde spiercellen hebben echter ook nog een andere functie, die essentieel is voor het behoud van de structuur van glad spierweefsel, te weten de synthese van extracellulaire matrix componenten. Om deze sterk verschillende functies te kunnen vervullen hebben gladde spiercellen de mogelijkheid om verschillende gedaanten of 'fenotypes' aannemen. Er bestaan zogenoemde contractiele en synthetische fenotypes. Als dat nodig is, bijvoorbeeld na beschadiging van glad spierweefsel, kunnen gladde spiercellen van het ene fenotype het andere fenotype aannemen via een proces dat fenotypische modulatie wordt genoemd. Ook als gladde spiercellen worden gekweekt treedt modulatie naar een synthetisch fenotype op. Het precieze fenotype van de gladde spiercel is echter niet alleen afhankelijk van de lokale omstandigheden. De grote verscheidenheid aan gladde spier fenotypes vindt ook zijn oorsprong in het feit dat gladde spiercellen uit verschillende kiembanen ontstaan, waaronder het mesoderm, de neurale lijst en het pro-epicardiaal orgaan.

De verschillende gladde spiercel fenotypes alsook de verschillende ontwikkelingsen differentiatie-stadia van gladde spiercellen worden in de praktijk gekarakteriseerd aan de hand van 1) morfologische kenmerken van de cellen, 2) proliferatie- en migratie eigenschappen van de cellen, en 3) expressie niveaus van verschillende zogenaamde gladde spier merker eiwitten. Veel van de vandaag bekende gladde spiercel merker eiwitten, zoals bijvoorbeeld gladde spier $\alpha$-actine, gladde spier myosine zware keten isovormen, SM22 $\alpha$ en smootheline, zijn karakteristiek voor het contractiele fenotype.

Hoe de differentiatie van de gladde spiercel en het gladde spiercel fenotype worden gereguleerd op het moleculaire niveau is nog niet volledig doorgrond. Er zijn echter wel verscheidene factoren bekend die het fenotype en de differentiatie beinvloeden. Platelet derived growth factor is een voorbeeld van een factor die de vroege differentiatie en de modulatie van het contractiele naar het synthetische fenotype stimuleert. Transforming growth factor- $\beta$ (TGF- $\beta$ ), retinoïden en heparine daarentegen stimuleren het contractiele fenotype. Naast deze biochemische factoren beïnvloeden ook fysische factoren het gladde spiercel fenotype. Hieronder vallen onder andere interacties met andere cellen, de organisatie van de extracellulaire matrix om de cel heen, en de druk en wrijving die op de gladde spiercel inwerken.

De verschillende cellijnen en muismodellen die zijn ontwikkeld om de regulatie van het gladde spiercel fenotype en de gladde spiercel differentiatie te kunnen bestuderen hebben uitgewezen dat de transcriptie factor serum response factor (SRF) hierbij een belangrijke rol speelt. $\mathrm{Na}$ interactie met verschillende cofactoren 
bindt SRF aan een DNA element dat CArC box wordt genoemd, en dat gevonden wordt in de promoters van bijna alle genen die alleen in gladde spiercellen tot expressie komen. Deze binding is essentieel voor de transcriptie van deze genen. Andere belangrijke regulerende elementen in deze genen zijn het TGF- $\beta$ controle element, de CAGA box en E-boxen. Recent is bovendien duidelijk geworden dat de chromatine structuur en alternatieve splicing belangrijke regulerende mechanismes zijn voor de bepaling van het gladde spiercel fenotype.

Zowel contractiele als synthetische gladde spiercellen maken eiwitten die exclusief betrokken zijn bij òf de contractiele òf de synthetische functie. Voor de contractiele gladde spiercel gaat het hierbij om bijvoorbeeld gladde spier $\alpha$-actine, gladde spier myosine isovormen en eiwitten die een interactie aangaan met deze eiwitten, zoals gladde spier calponine, $S M 22 \alpha$ en smoothelines. Deze eiwitten dragen bij aan de specifieke kenmerken van de gladde spiercel contractie, die grotendeels gereguleerd wordt door het fosforylerings niveau van de lichte ketens van het myosine. Dit fosforylerings niveau hangt weer af de activiteit van de enzymen myosine lichte keten kinase en myosine lichte keten fosfatase, die op $\mathrm{Ca}^{2+}$ afhankelijke en -onafhankelijke manieren worden gereguleerd. Eiwitten die betrokken zijn bij de functies van de synthetische gladde spiercel zijn de extracellulaire matrix eiwitten, waaronder collageen en elastine, en enzymen als matrix metalloproteinases en hun remmers, die de hoeveelheid en de structuur van de extracellulaire matrix controleren.

Uit het bovenstaande moge duidelijk zijn dat het onderzoek naar de differentiatie, regulatie en functie van gladde spiercellen grotendeels gebaseerd is op de karakterisatie van gladde spiercel merker eiwitten. Eén van deze merker eiwitten, smootheline, is het centrale onderwerp van dit proefschrift. Aan het begin van de studies die beschreven staan in dit proefschrift was bekend dat er twee vormen van smootheline zijn: één van $59 \mathrm{kDa}$ die voornamelijk gemaakt wordt door viscerale gladde spiercellen (smootheline-A), en één van $110 \mathrm{kDa}$ die vooral in de gladde spiercellen van de vaten wordt aangetroffen (smootheline-B). Ook was bekend dat smoothelines structurele eiwitten zijn met een C-terminaal actine bindend domein, die worden gecodeerd door een gen dat alleen actief is in contractiele gladde spiercellen. Om de bruikbaarheid van smoothelines voor het onderzoek naar de differentiatie, regulatie en functie van gladde spiercellen verder te vergroten werden studies opgezet naar de expressie, regulatie en functie van smoothelines.

De expressie van smootheline-B in vergelijking tot die van andere contractiele gladde spiercel merker eiwitten werd bestudeerd in een nieuw in vitro gladde spiercel model, gevormd door coronaire gladde spiercellen van het varken (hoofdstuk 2). Het bleek dat smootheline-B veel gevoeliger was voor de fenotypische modulatie van deze gladde spiercellen dan de andere gladde spier merker eiwitten. De sterke afname van de smootheline-B niveaus gedurende de 
modulatie kon worden geremd door heparine aan het kweekmedium toe te voegen. TGF- $\beta$ en retinoïden daarentegen hadden geen effect op het smootheline-B gehalte. In tegenstelling tot in andere gladde spiercellen in vitro kon in de cellen van ons modelsysteem smootheline-B nog worden aangetoond na enige tijd kweken. Hiervan werd gebruik gemaakt om de subcellulaire localisatie van smootheline-B te bestuderen. Smootheline-B bleek te colocaliseren met gladde spier $\alpha$-actine, dat zich in de stress fibers van gekweekte gladde spiercellen bevindt. Het bleek daarentegen niet te colocaliseren met intermediaire filamenten.

Vervolgens werd het expressiepatroon van smoothelines gedurende de ontwikkeling van het kippenembryo bestudeerd (hoofdstuk 3). Smoothelines konden voor het eerst worden aangetoond op Hamburger-Hamilton stadium 17, en wel in het primitieve hart en het myotoom van de somieten. De genoemde weefsels brachten een nog onbekende isovorm tot expressie, die we smootheline-C hebben gedoopt. Dit smootheline-C bleek een variant van smootheline-A te zijn. Een interessante observatie was dat zowel vasculaire (vanaf stadium 20) als viscerale (vanaf stadium 29) gladde spiercellen smootheline-B maakten tijdens de embryogenese. Pas rond het tijdstip van uitkomen van het ei werd de normale smootheline-A isovorm gemaakt in de viscerale weefsels, en hield de expressie van smootheline-B daar op. Op dat moment kon er ook geen smootheline-C in het hart of de somieten meer worden gevonden. Dit expressie patroon duidt erop dat de diverse smootheline varianten verschillende functies hebben bij de gladde spiercel contractie.

$\mathrm{Na}$ de bestudering van het expressie patroon van de smoothelines werd onderzocht hoe hun expressie werd gereguleerd (hoofdstuk 4). De belangrijkste bevinding was dat er twee ver uit elkaar liggende promoters in het smootheline gen aanwezig zijn die de transcriptie van de verschillende smootheline isovormen aansturen. De smootheline-B promoter ligt direct voor het eerste smootheline-B exon, terwijl de smootheline-A promoter midden in het gen ligt, en wel direct voor exon 10. Verdere analyse van de smootheline promoters (hoofdstuk 5) wees uit dat de smootheline-B promoter sterker is dan de smootheline-A promoter. Bovendien werd het duidelijk dat de transcriptie van smootheline-A deels gereguleerd wordt door binding van SRF aan de CArG box. Een belangrijke cofactor van SRF, myocardine, verhoogde de smootheline-A promoter activiteit, maar had nauwelijks effect op de smootheline-B promoter activiteit. Smootheline-A en smootheline-B worden dus op verschillende manieren gereguleerd. Ook zijn ze voor hun expressie niet compleet afhankelijk van CArG boxen, zoals de meeste andere gladde spiercel merker genen dat zijn. Naast deze regulatie op transcriptieniveau bleek de expressie van smootheline isovormen ook via alternatieve splicing van het actine bindend domein gecontroleerd te worden.

Omdat de functie van smoothelines moeilijk te bepalen was met behulp van in vitro experimenten moest een smootheline knockout muis worden ontwikkeld, die 
functionele studies naar de smoothelines in een levend organisme mogelijk zou maken. Voorafgaande aan dit project werd eerst het muizen smootheline gen gekloneerd, in kaart gebracht en gesequenced (hoofdstuk 6). In situ hybridisatie wees uit dat het muizen smootheline gen op chromosoom 11, band A2-A3 ligt. Het bevat 21 exonen die hetzelfde zijn georganiseerd als de humane smootheline exonen. Het verwijderen van de exonen 18 tot en met 20 van het muize smootheline gen, die coderen voor het actine bindende domein, leverde functionele smootheline knockout muizen op (hoofdstuk 7). Deze muizen stierven op jonge leeftijd aan pathologieën van verschillende organen, veroorzaakt door een dysfunctie van de gladde spiercellen. Ze ontwikkelden diverticuli in de darmen, leden aan hydronephrose en cholestase, en waren onvruchtbaar. Bloedvaten van smootheline knockout muizen hadden een dunnere spierwand. Bovendien was het vermogen tot samentrekken en relaxeren van de bloedvaten sterk verminderd. Ook de contractiliteit van de darmen leek aangedaan. De voorlopige conclusie van dit werk is dan ook dat smoothelines cruciaal zijn voor het fysiologisch functioneren van gladde spieren en dat ze structureel betrokken zijn bij de gladde spiercel contractie.

In het laatste hoofdstuk van het proefschrift worden verschillende experimenten voorgesteld die kunnen leiden tot een beter inzicht in de regulatie en functie van smoothelines. De belangrijkste hiervan zijn de ontwikkeling van transgene reporter muizen om de regulatie van de smootheline transcriptie in vivo te kunnen bestuderen, en de analyse van het effect van mutaties van de E-boxen in de smootheline promoters op hun activiteit. Een betere bestudering van de karakteristieken van de gladde spiercel contractie, proliferatie en merker eiwit expressie in de smootheline knockout modellen zal bijdragen aan ons begrip van zowel de functie van de smoothelines als de regulatie van gladde spiercel fenotypes en de werking van het contractiele apparaat van de gladde spiercel. 
Bedankt! 
Van alle dingen die gedaan moesten worden voor het maken van dit proefschrift was het schrijven van een dankwoord misschien nog wel het moeilijkste, en tegelijkertijd voelde het als éen van de belangrijkste. Zonder alle mensen die me hebben geholpen gedurende de afgelopen jaren zou er ten eerste niet genoeg lol zijn geweest en ten tweede gewoon niet genoeg zijn gebeurd. Bovendien is dit het enige deel van het boekje dat iedereen ook echt leest......

Bedanken is dus een serieuze zaak, en geen spelletje. Datzelfde geldt voor promotieonderzoek, hoewel, als je er goed over nadenkt, zijn er toch wel wat parallellen te trekken met datgene wat ik de afgelopen jaren na het werk zoveel gedaan heb, namelijk het spelen van 'de Kolonisten van Catan'.

Eerst de voornaamste gelijkenis: net zo min als je een spelletje alleen speelt, maak je een proefschrift alleen. Het speelveld van het proefschrift werd opgebouwd bij de collega's van Moleculaire Celbiologie en Genetica. Vanaf de dag dat ik daar begon (met bijna direct het befaamde dagje uit op het paard) heb ik het er naar m'n zin gehad. Ik werd geïntroduceerd tot de belangrijkste grondstoffen die benodigd zijn voor een allround onderzoeker.

Guillaume bracht de essentiële grondstoffen: DNA, eiwitten, bacteriën en virussen. Maar hij bracht veel meer dan dat. Ik heb geen idee hoe het te omschrijven, maar zonder zijn temperament, 'rijstijl' en 'navigatietalent', losse manier van begeleiden, 'muziek'voorkeur, inzet, 'computervaardigheden', recordsnelle correcties van manuscripten, grappen en voetbalkennis zou hij het nooit tot de 'meest geschrokken Baas van de Dag' in de Arbeidsvitaminen hebben geschopt. Guillaume, bedankt voor alles wat je voor me hebt gedaan en Jeanette, bedankt voor al die keren dat het doornemen van een manuscript vergezeld ging van een geweldige maaltijd vooraf! Frank, Jannie, Hans en Erika gaven het goede voorbeeld qua plezier en inzet. Sindsdien probeer ik elk lab zo gezellig te maken als het was in het lab waarin ik met hen begon. Jos en Mieke gaven me de beschikking over een andere cruciale grondstof voor m'n promotiespel: cellen. En Wiel leerde me de officiële spelregels. Frans, Francien, Bert, Marcel, Nancy, Hans, Jorike, Ton, Miriam, Helma, Marie-Hélène, Annick, Ernst-Jan, Stefan, Sandra, Manon, Joris, Maurice, Elena, Edith, Barbie, Wendy en Monique waren de altijd voor hulp aanwezige kaders van het spel. Allemaal ontzettend bedankt!

Halverwege m'n AlO-schap kwam de eerste speluitbreiding, met een echte Hollandse invasie uit Leiden. Uiteindelijk werden Guillaume en ik geannexeerd, en gingen we verder onder de Moleculaire Genetica vlag. Dit zou nooit zo vlotjes zijn verlopen zonder de geweldige organisatie van Patrick, Monique en Wilma. Achter hen staat Marten, die ik graag wil bedanken omdat hij een veilige haven was voor m'n onderzoek.

Net zoals een gemiddeld potje Kolonisten ontwikkelde Moleculaire Genetica zich razendsnel, alleen niet dankzij ontwikkelingskaarten, maar dankzij leuke mensen. Dat waren Le, Menno, Marion, Inge, Stijn, Mathijs, Olivier, Hanneke, Céline, Frank, Vivian, Iris, Ronit, Anne, Willem, Yvonne, en op het laatst Susan, Jan, Debby, Joost, 
Maurice, Will, Roy en Danielle. Enorm bedankt voor alle lol die we hebben gehad, voor alle goeie suggesties en kritische opmerkingen, voor het luisteren naar verhalen over Zwientie tikken, en vooral bedankt dat jullie me hebben laten zingen op het lab, zelfs die ene keer dat ik niet m'n dag had.

Tijdens het spel spelen een aantal figuren nog een bijzondere rol. Zo hebben we de handelaar, in mijn geval Pieter, die ik graag wil bedanken voor het feit dat hij niet alleen financieel bijdroeg aan m'n onderzoek, maar er met zijn resultaatgerichtheid en andere invalshoeken ook voor zorgde dat we scherp bleven.

Er zijn ook kapers op de kust, de struikrovers en piraten. De piraten kunnen je een luur draaien als je niet snel genoeg bent, en helaas heb ik dat aan den lijve ondervonden tijdens mijn promotiespel door de Duitse groep die ons te snel af was met het publiceren van het 'smootheline-B verhaal'. Van de andere kant waren er ook struikrovers, oftewel de leden van de beoordelingscommissie, die zich kritisch hebben gebogen over de lange tekst van dit manuscript, en het mes (nog) niet hebben getrokken. Bedankt daarvoor! Ik hoop dat jullie niet alsnog mijn kaarten zullen afpakken of het verloop van het spel zullen blokkeren.

Heel belangrijk tijdens het spel zijn de ridders oftewel de paranimfen, Victor en Petra. Zij stonden me bij, niet alleen in het lab en op de AlO-kamer, maar ook daarbuiten. Zij weten als enige wat het betekent om de AlO's van 'de snor' te zijn. Ik vind het ongelooflijk gaaf dat ze erbij zijn straks!

Dan de langste handelsroute, die me achtereenvolgens naar Zwitserland, Canada en Amerika voerde.

Dear Giulio, Thomas, Marie-Luce, Christine, Sophie, and all the others, thank you for the wonderful time in Geneva. I will never forget what you have done for me and hopefully I will never forget what you taught me. Merci beaucoup (my French still has not improved).

Geoff Pickering, Edward, Shaohua, and Caroline, thanks for helping me tackle the promoter assays with your cells and viruses. It is too bad that it has not led to a manuscript yet, but the effort is much appreciated!

Finally, Jan, Darren, Sandy, Babu, Gazi, and Shelly, I don't know how to express my gratitude towards you. Without you there wouldn't have been a chapter 7 at all. Knowing that I was welcome for a second time in such an excellent laboratory strengthened my confidence. Darren and Betsy, a special thanks goes out to you for knowing me and still offering your place for me to stay. It is good to know that you are still playing Settlers of Catan!

Ook dichterbij huis waren er veel mensen waar we mee samen hebben gewerkt. Carlie, Marco, Gerard, Jo, bedankt dat jullie jullie kennis en vaardigheden met me wilden 'ruilen'! Joe, thanks for the fruitful collaboration, it was a pleasure working and communicating with you! Alle mensen van het CPV, bedankt voor het zo goed verzorgen van de muizen, zonder jullie wisten we waarschijnlijk nog niet waar al 
die kleintjes waren gebleven. Martijn, Mandy, Erik, Ruud, Kim, Alexandra, Diane, Marjolein, allemaal stagiaires die me hebben geholpen, en zonder wie er nooit zoveel gedaan zou kunnen zijn. Zonder de geweldige hulp van Berry en Henri zouden er een stuk minder sequenties zijn bepaald, en zou m'n computer nu waarschijnlijk nog steeds niet aan het netwerk zijn geknoopt, bedankt!

Tijdens m'n promotiespel waren er een heleboel toeschouwers, die zorgden voor broodnodig ontspanning ende vermaeck: vrienden, familie, en andere AIO's. Patrick, Raimond, Joanda, Marcel, Christiaan, Sonja, Edwin, Jan, Arnold, Roy, ik vind het erg bijzonder en leuk dat we als Raaltese vrienden, al die jaren na de middelbare school, nog steeds gewoon bij elkaar kunnen komen op een zaterdagavond en kunnen ouwehoeren alsof we 15 zijn. Ik beloof dat ik jullie nooit meer een endoplasmatisch reticulum zal laten tekenen met Party en Co!

Marian, Mark, Annemarie, Aldrik, Martine, Fleur, Patrick, Alinda, Marije, Rien, Auke, Renate, Helicasers, jullie hebben ervoor gezorgd dat ik me zo thuis ben gaan voelen in Maastricht dat ik me bijna niet kan voorstellen ooit weg te zullen gaan, ook al zijn de meesten van jullie allang weer verdwenen.

Anita, Michel, Bianca, Miriam, Lorraine, Rudy, Paula, Lars, ik heb genoten van alle gezellige (AlO-)etentjes en spelletjesavonden, laat ze niet in het slop geraken!

Alle ooms en tantes, neven en nichten Rensen en Kievitsbosch en iedereen van de familie Niessen en Nijsen (vooral Jan, Jeannie en Hanneke natuurlijk), bedankt voor de interesse en aandacht als ik weer eens probeerde uit te leggen waar ik mee bezig was of hoe ver ik er mee was.

Matthijs, Veerle en Peter, fantastisch dat jullie me niet het gevoel gaven dat ik niet aan m'n proefschrift kon werken in jullie (en nu ook mijn) frizzled-wereld. Mede daardoor is het nu eindelijk af!

Al die tijd heeft het spel gelegen op een massieve, geduldige en vanzelfsprekende tafel, die gevormd wordt door m'n familie. Pa en ma, Marc, Ruben, Anneke en Ans, zonder jullie zou het promotiespel als een kaartenhuis zijn ingestort. Bedankt voor de steun op afstand en dichtbij. -

Lieve Petra, jij begon pas mee te spelen toen ik al halverwege het spel was. Ik had nooit gedacht dat we ooit een 'echt' team zouden vormen! Maar wat ik wel weet is dat het ervoor zorgt dat ik me een winnaar voel. Het volgende potje is voor jou!

\section{Sander}

P.S. Ik vrees dat er geen 'uitbreiding' zal volgen om degenen die ik ongetwijfeld vergeten ben in dit dankwoord alsnog de plaats en eer te geven die hen toekomt. Voor iedereen die dus ten onrechte van het bord is geveegd: sorry en toch bedankt! 


\section{Curriculum vitae List of publications}


Sander Rensen was born in Deventer on June 10, 1975. He grew up in the small village of Raalte in the eastern part of the Netherlands, spending most of his time playing at his father's farm.

After completing secondary school at the Florens Radewijns College in Raalte in 1993, he moved to Maastricht to study Health Sciences at the Universiteit Maastricht. Initially, he wanted to specialize in Environmental Health Sciences, but fortunately he was kept from this mistake during the course of the study. The introduction to Molecular Biology during the practical trainings of the Biological Health Sciences specialization got him enthusiastic, and when he had to decide about his traineeship, the choice was easy. The opportunity to get his training in the lab of Guillaume van Eys at the department of Molecular Cell Biology came at the right time, and it was then that the smoothelins first stole his heart. After a little detour in the lab of Professor Giulio Gabbiani in Geneva, where he got acquainted with cell culture in the mountains and had a lot of fun learning a mix of French, Schwyzertütsch, and Italian, he graduated cum laude in 1998.

Subsequently, big promises and the prospect of even bigger money enticed him into working as a Junior Scientist at IntroGene B.V. in Leiden. Soon enough though, he discovered that trying to stuff genes into adenoviruses for the purpose of gene function discovery was not his idea of a nice job. After a visit to the van Eys lab, the plans to become a PhD student gained momentum, and later in 1999, he started unraveling the fundamentals of smoothelins in the framework provided by the Cardiovascular Research Institute Maastricht.

Since then, four years of research led him to believe that it is true that the more you know, the more you discover that you don't know enough, hence the more you want to know. During these years, working visits to the lab of Professor Geoff Pickering at the John P. Robarts Research Institute in London, Canada (October/November 2000) and the lab of Dr. Jan van Deursen at the Mayo Clinic in Rochester (MN), USA (October-December 2001; November-December 2002) contributed to not only the scientific quality of his work, but also to the notion that the American way of life was not his way of life.

Therefore, he has just started with a challenging project in the Netherlands, at the department of Pharmacology and Toxicology of the Universiteit Maastricht. The elucidation of the role of the wnt/frizzled/dishevelled signaling pathway in the recruitment of myofibroblasts into myocardial scar tissue should keep him busy until some time in 2006. 


\section{Full papers}

Niessen P, Clément S, Fontao L, Chaponnier C, Teunissen B, Rensen S, van Eys G, Gabbiani $G$. Biochemical evidence for interaction between smoothelin and filamentous actin. Experimental Cell Research 292: 170-178 (2004).

Rensen S, Thijssen V, De Vries C, Doevendans P, Detera-Wadleigh S, Van Eys G. Expression of the smoothelin gene is mediated by alternative promoters. Cardiovascular Research 55: 850-863 (2002).

De Ruiter M, Rensen S, Coolen G, Hierck B, Bergwerff M, Debie W, Gittenberger-de Groot $A$, van Eys $G$. Smoothelin expression during chicken embryogenesis: Detection of an embryonic isoform. Developmental Dynamics 221: 460-463 (2001).

Rensen S, Merkx G, Doevendans P, Geurts van Kessel A, van Eys G. Structure and chromosome location of Smtn, the mouse smoothelin gene. Cytogenetics and Cell Genetics 89: 225-229 (2000).

Christen T, Bochaton-Piallat ML, Neuville P, Rensen S, Redard M, van Eys G, Gabbiani G. Cultured porcine coronary artery smooth muscle cells. A new model with advanced differentiation. Circulation Research 85: 99-107 (1999).

Van Eys G, de Vries C, Rensen S, Thijssen V, Verkaar E, Coolen G, Debie W, de Ruiter M, Wadleigh-Detera S. Smoothelins: One gene, two proteins, three muscle cell types so far. In: Cardiovascular Specific Gene Expression; edited by Doevendans P, Reneman R, van Bilsen M. p. 49-67 (1999).

\section{Abstracts}

Rensen S, Niessen P, van Deursen J, Fazzi G, Janssen B, van Eys G, De Mey J. Inactivation of the smoothelin gene leads to reduced arterial reactivity. $8^{\text {th }}$ Annual Meeting of the European Council for Blood Pressure and Cardiovascular Research. Hypertension 42 (4) p628, 2.05 (2003); oral presentation.

Rensen S, Niessen P, van Deursen J, Gijbels $M$, Doevendans P, Hofker M, De Mey J, van Eys $G$. Inactivation of the smoothelin gene leads to aberrant vascular functioning. $4^{\text {th }}$ Annual Conference on Arteriosclerosis, Thrombosis and Vascular Biology 2003; Arteriosclerosis, Thrombosis, and Vascular Biology 23 (5), p a-6, 32 (2003).

Rensen S, Niessen P, Doevendans P, van Deursen J, Hofker M, van Eys G. Analysis of the function and regulation of smoothelins, contractile smooth muscle specific proteins. European Cytoskeleton Forum meeting 2002; oral presentation. 
Rensen S, Niessen P, Doevendans P, Hofker M, Pickering G, de Ruiter M, Gabbiani G, van Eys $G$. Function and regulation of smoothelins, markers of contractile smooth muscle cells. European Cytoskeleton Forum meeting 2001.

Rensen S, Doevendans P, van Eys G. Expression of smoothelin isoforms is controlled by two physically separated promoters. International Vascular Biology meeting 2000; oral presentation; Journal of Submicroscopic Cytology and Pathology 32 (3), p 4865094 (2000).

Rensen S, Doevendans P, Debie W, van Eys G. Molecular and cell biological aspects of smoothelins. International Society for Heart Research meeting 1999. Journal of Molecular and Cellular Cardiology 31 (6), p A116 Sa46 (1999).

Rensen S, Verkaar E, Debie W, de Vries C, van Eys $G$. A vascular-specific isoform of smoothelin. Journal of Molecular and Cellular Cardiology 29 (5), p A42 (1997). 
References 
1. Owens GK. Regulation of differentiation of vascular smooth muscle cells. Physiol Rev. 1995;75:487-517.

2. Letunic I, Goodstadt L, Dickens NJ, Doerks T, Schultz J, Mott R, Ciccarelli F, Copley RR, Ponting CP, Bork P. Recent improvements to the SMART domain-based sequence annotation resource. Nucleic Acids Res. 2002;30:242-244.

3. Hao H, Ropraz P, Verin V, Camenzind E, Geinoz A, Pepper MS, Gabbiani G, Bochaton-Piallat ML. Heterogeneity of smooth muscle cell populations cultured from pig coronary artery. Arterioscler Thromb Vasc Biol. 2002;22:1093-1099.

4. Chamley-Campbell J, Campbell GR, Ross R. The smooth muscle cell in culture. Physiol Rev. 1979;59:1-61.

5. Horowitz A, Menice CB, Laporte R, Morgan KG. Mechanisms of smooth muscle contraction. Physiol Rev. 1996;76:967-1003.

6. Worth NF, Rolfe BE, Song J, Campbell GR. Vascular smooth muscle cell phenotypic modulation in culture is associated with reorganisation of contractile and cytoskeletal proteins. Cell Motil Cytoskeleton. 2001;49:130-145.

7. Yamada KM, Geiger B. Molecular interactions in cell adhesion complexes. Curr Opin Cell Biol. 1997;9:76-85.

8. Small N, Gimona M. The cytoskeleton of the vertebrate smooth muscle cell. Acta Physiol Scand. 1998;164:341-348.

9. Bond M, Somlyo AV. Dense bodies and actin polarity in vertebrate smooth muscle. $J$ Cell Biol. 1982;95:403-413.

10. Small NV, Rottner K, Kaverina I, Anderson KI. Assembling an actin cytoskeleton for cell attachment and movement. Biochim Biophys Acta. 1998;1404:271-281.

11. Stromer MH, Mayes MS, Bellin RM. Use of actin isoform-specific antibodies to probe the domain structure in three smooth muscles. Histochem Cell Biol. 2002;118:291-299.

12. Bochaton-Piallat ML, Ropraz P, Gabbiani F, Gabbiani G. Phenotypic heterogeneity of rat arterial smooth muscle cell clones. Implications for the development of experimental intimal thickening. Arterioscler Thromb Vasc Biol. 1996;16:815-820.

13. Li S, Fan YS, Chow $L H$, Van Den Diepstraten C, van Der Veer E, Sims SM, Pickering JG. Innate diversity of adult human arterial smooth muscle cells: cloning of distinct subtypes from the internal thoracic artery. Circ Res. 2001;89:517-525.

14. Lemire JM, Covin CW, White S, Giachelli CM, Schwartz SM. Characterization of cloned aortic smooth muscle cells from young rats. Am J Pathol. 1994;144:1068-1081.

15. Bochaton-Piallat ML, Gabbiani F, Redard M, Desmouliere A, Gabbiani G. Apoptosis participates in cellularity regulation during rat aortic intimal thickening. Am J Pathol. 1995;146:1059-1064.

16. Brittingham J, Phiel C, Trzyna WC, Gabbeta V, McHugh KM. Identification of distinct molecular phenotypes in cultured gastrointestinal smooth muscle cells. Gastroenterology. 1998;115:605617.

17. Page KL, Celia G, Leddy G, Taatjes DJ, Osol G. Structural remodeling of rat uterine veins in pregnancy. Am J Obstet Gynecol. 2002;187:1647-1652.

18. Christen $T$, Verin V, Bochaton-Piallat $M$, Popowski Y, Ramaekers $F$, Debruyne $P$, Camenzind $E$, van Eys $G$, Gabbiani $G$. Mechanisms of neointima formation and remodeling in the porcine coronary artery. Circulation. 2001;103:882-888.

19. Maeng $M$, Mertz $H$, Nielsen S, Van Eys GJ, Rasmussen K, Espersen GT. Adventitial myofibroblasts play no major role in neointima formation after angioplasty. Scand Cardiovasc J. 2003;37:34-42.

20. Frid MG, Shekhonin BV, Koteliansky VE, Glukhova MA. Phenotypic changes of human smooth muscle cells during development: late expression of heavy caldesmon and calponin. Dev Biol. 1992;153:185-193.

21. Glukhova MA, Kabakov AE, Frid MG, Ornatsky OI, Belkin AM, Mukhin DN, Orekhov AN, Koteliansky VE, Smirnov VN. Modulation of human aorta smooth muscle cell phenotype: a study of musclespecific variants of vinculin, caldesmon, and actin expression. Proc Natl Acad Sci U S A. 1988;85:9542-9546. 
22. Johnson JL, van Eys GJ, Angelini GD, George SJ. Injury induces dedifferentiation of smooth muscle cells and increased matrix-degrading metalloproteinase activity in human saphenous vein. Arterioscler Thromb Vasc Biol. 2001;21:1146-1151.

23. van der Loop FT, Schaart G, Timmer ED, Ramaekers FC, van Eys GJ. Smoothelin, a novel cytoskeletal protein specific for smooth muscle cells. J Cell Biol. 1996;134:401-411.

24. Neuville $P$, Geinoz A, Benzonana $G$, Redard $M$, Gabbiani $F$, Ropraz $P$, Gabbiani $G$. Cellular retinolbinding protein-1 is expressed by distinct subsets of rat arterial smooth muscle cells in vitro and in vivo. Am J Pathol. 1997;150:509-521.

25. Blindt $R$, Krott $N$, Hanrath $P$, vom Dahl J, van Eys $G$, Bosserhoff AK. Expression patterns of integrins on quiescent and invasive smooth muscle cells and impact on cell locomotion. $J$ Mol Cell Cardiol. 2002;34:1633-1644.

26. Hultgardh-Nilsson A, Lovdahl C, Blomgren $K$, Kallin B, Thyberg J. Expression of phenotype- and proliferation-related genes in rat aortic smooth muscle cells in primary culture. Cardiovasc Res. 1997:34:418-430.

27. Blindt $R$, Zeiffer U, Krott $N$, Filzmaier $K$, Voss $M$, Hanrath P, vom Dahl J, Bosserhoff AK. Upregulation of the cytoskeletal-associated protein Moesin in the neointima of coronary arteries after balloon angioplasty: a new marker of smooth muscle cell migration? Cardiovasc Res. 2002;54:630-639.

28. Ko YS, Yeh HI, Haw M, Dupont E, Kaba R, Plenz G, Robenek H, Severs NJ. Differential expression of connexin 43 and desmin defines two subpopulations of medial smooth muscle cells in the human internal mammary artery. Arterioscler Thromb Vasc Biol. 1999;19:1669-1680.

29. Cizmeci-Smith $G$, Langan $E$, Youkey J, Showalter $U$, Carey DJ. Syndecan-4 is a primary-response gene induced by basic fibroblast growth factor and arterial injury in vascular smooth muscle cells. Arterioscler Thromb Vasc Biol. 1997;17:172-180.

30. Rolfe BE, Muddiman JD, Smith NJ, Campbell GR, Campbell JH. ICAM-1 expression by vascular smooth muscle cells is phenotype-dependent. Atherosclerosis. 2000;149:99-110.

31. Ueki N, Sobue K, Kanda K, Hada T, Higashino K. Expression of high and low molecular weight caldesmons during phenotypic modulation of smooth muscle cells. Proc Natl Acad Sci U S A. 1987;84:9049-9053.

32. Sekiguchi K, Kurabayashi M, Oyama $Y$, Aihara $Y$, Tanaka $T$, Sakamoto $H$, Hoshino $Y$, Kanda $T$, Yokoyama $T$, Shimomura $Y$, lijima $H$, Ohyama $Y$, Nagai $R$. Homeobox protein Hex induces

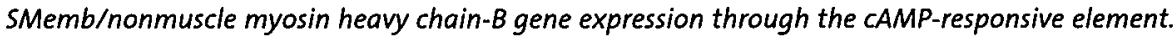
Circ Res. 2001;88:52-58.

33. Shanahan CM, Weissberg PL, Metcalfe JC. Isolation of gene markers of differentiated and proliferating vascular smooth muscle cells. Circ Res. 1993;73:193-204.

34. Lees D, Fabbrizio E, Mornet D, Pugnere D, Travo P. Parallel expression level of dystrophin and contractile performances of rat aortic smooth muscle. Exp Cell Res. 1995;218:401-404.

35. Obata H, Hayashi $K$, Nishida W, Momiyama T, Uchida A, Ochi $T$, Sobue K. Smooth muscle cell phenotype-dependent transcriptional regulation of the alpha1 integrin gene. J Biol Chem. 1997;272:26643-26651.

36. Koyama N, Seki J, Vergel S, Mattsson EJ, Yednock T, Kovach NL, Harlan JM, Clowes AW. Regulation and function of an activation-dependent epitope of the beta 1 integrins in vascular cells after balloon injury in baboon arteries and in vitro. Am J Pathol. 1996;148:749-761.

37. Yao CC, Breuss J, Pytela R, Kramer RH. Functional expression of the alpha 7 integrin receptor in differentiated smooth muscle cells. J Cell Sci. 1997;110 (Pt 13):1477-1487.

38. Bochaton-Piallat ML, Gabbiani F, Ropraz P, Gabbiani G. Cultured aortic smooth muscle cells from newborn and adult rats show distinct cytoskeletal features. Differentiation. 1992;49:175-185.

39. Chen J, Kitchen CM, Streb JW, Miano JM. Myocardin: a component of a molecular switch for smooth muscle differentiation. I Mol Cell Cardiol. 2002;34:1345-1356.

40. Uglow EB, Slater S, Sala-Newby GB, Aguilera-Garcia CM, Angelini GD, Newby AC, George SJ. Dismantling of Cadherin-Mediated Cell-Cell Contacts Modulates Smooth Muscle Cell Proliferation. Circ Res. 2003. 
41. Layne MD, Endege Wo, Jain MK, Yet SF, Hsieh CM, Chin MT, Perrella MA, Blanar MA, Haber E, Lee ME. Aortic carboxypeptidase-like protein, a novel protein with discoidin and carboxypeptidase-like domains, is up-regulated during vascular smooth muscle cell differentiation. J Biol Chem. 1998;273:15654-15660.

42. Kashiwada $K$, Nishida $W$, Hayashi K, Ozawa K, Yamanaka $Y$, Saga $H$, Yamashita $T$, Tohyama M, Shimada S, Sato $K$, Sobue $K$. Coordinate expression of alpha-tropomyosin and caldesmon isoforms in association with phenotypic modulation of smooth muscle cells. J Biol Chem. 1997;272:1539615404.

43. Hsieh CM, Yoshizumi M, Endege WO, Kho CJ, Jain MK, Kashiki S, de los Santos $R$, Lee WS, Perrella MA, Lee ME. APEG-1, a novel gene preferentially expressed in aortic smooth muscle cells, is downregulated by vascular injury. J Biol Chem. 1996;271:17354-17359.

44. Jain MK, Fujita KP, Hsieh CM, Endege WO, Sibinga NE, Yet SF, Kashiki S, Lee WS, Perrella MA, Haber $E$, Lee ME. Molecular cloning and characterization of SMLIM, a developmentally regulated LIM protein preferentially expressed in aortic smooth muscle cells. J Biol Chem. 1996;271:1019410199.

45. Hungerford JE, Little CD. Developmental biology of the vascular smooth muscle cell: building a multilayered vessel wall. J Vasc Res. 1999;36:2-27.

46. Roy SG, Nozaki Y, Phan SH. Regulation of alpha-smooth muscle actin gene expression in myofibroblast differentiation from rat lung fibroblasts. Int J Biochem Cell Biol. 2001;33:723-734.

47. Buniatian GH, Gebhardt R, Mecke D, Traub P, Wiesinger H. Common myofibroblastic features of newborn rat astrocytes and cirrhotic rat liver stellate cells in early cultures and in vivo. Neurochem Int. 1999;35:317-327.

48. Amberger A, Bauer $H$, Tontsch U, Gabbiani $G$, Kocher $O$, Bauer HC. Reversible expression of sm alpha-actin protein and $5 m$ alpha-actin $m R N A$ in cloned cerebral endothelial cells. FEBS Lett. 1991;287:223-225.

49. Sawtell NM, Lessard $U$. Cellular distribution of smooth muscle actins during mammalian embryogenesis: expression of the alpha-vascular but not the gamma-enteric isoform in differentiating striated myocytes. J Cell Biol. 1989;109:2929-2937.

50. McHugh KM. Molecular analysis of smooth muscle development in the mouse. Dev Dyn. 1995;204:278-290.

51. Landerholm TE, Dong XR, LU J, Belaguli NS, Schwartz RJ, Majesky MW. A role for serum response factor in coronary smooth muscle differentiation from proepicardial cells. Development. 1999;126:2053-2062.

52. Browning CL, Culberson DE, Aragon IV, Fillmore RA, Croissant JD, Schwartz RJ, Zimmer WE. The developmentally regulated expression of serum response factor plays a key role in the control of smooth muscle-specific genes. Dev Biol. 1998;194:18-37.

53. Babij $P$. Tissue-specific and developmentally regulated alternative splicing of a visceral isoform of smooth muscle myosin heavy chain. Nucleic Acids Res. 1993;21:1467-1471.

54. Kelley $C A$, Takahashi $M, Y u J H$, Adelstein RS. An insert of seven amino acids confers functional differences between smooth muscle myosins from the intestines and vasculature. I Biol Chem. 1993;268:12848-12854.

55. Aikawa M, Sivam PN, Kuro-o M, Kimura K, Nakahara K, Takewaki S, Ueda M, Yamaguchi $H$, Yazaki $Y$, Periasamy $M$, et al. Human smooth muscle myosin heavy chain isoforms as molecular markers for vascular development and atherosclerosis. Circ Res. 1993;73:1000-1012.

56. Babu GJ, Warshaw DM, Periasamy M. Smooth muscle myosin heavy chain isoforms and their role in muscle physiology. Microsc Res Tech. 2000;50:532-540.

57. Miano $J M$, Cserjesi $P$, Ligon $K L$, Periasamy $M$, Olson EN. Smooth muscle myosin heavy chain exclusively marks the smooth muscle lineage during mouse embryogenesis. Circ Res. 1994;75:803812.

58. Solway J, Seltzer J, Samaha FF, Kim S, Alger LE, NiU Q, Morrisey EE, Ip HS, Parmacek MS. Structure and expression of a smooth muscle cell-specific gene, SM22 alpha. I Biol Chem. 1995;270:1346013469. 
59. Regan CP, Adam PJ, Madsen CS, Owens GK. Molecular mechanisms of decreased smooth muscle differentiation marker expression after vascular injury. J Clin Invest. 2000;106:1139-1147.

60. Schildmeyer LA, Braun R, Taffet $G$, Debiasi $M$, Burns AE, Bradley A, Schwartz RJ. Impaired vascular contractility and blood pressure homeostasis in the smooth muscle alpha-actin null mouse. Faseb J. 2000;14:2213-2220.

61. Gabbiani $G$, Kocher $O$, Bloom WS, Vandekerckhove J, Weber K. Actin expression in smooth muscle cells of rat aortic intimal thickening, human atheromatous plaque, and cultured rat aortic media. $J$ Clin Invest. 1984;73:148-152.

62. Kovacs AM, Zimmer WE. Molecular cloning and expression of the chicken smooth muscle gammaactin MRNA. Cell Motil Cytoskeleton. 1993;24:67-81.

63. Kovacs AM, Zimmer WE. Cell-specific transcription of the smooth muscle gamma-actin gene requires both positive- and negative-acting cis elements. Gene Expr. 1998;7:115-129.

64. Oian J, Kumar A, Szucsik JC, Lessard UL. Tissue and developmental specific expression of murine smooth muscle gamma-actin fusion genes in transgenic mice. Dev Dyn. 1996;207:135-144.

65. Babu GJ, Loukianov E, Loukianova T, Pyne GJ, Huke S, Osol G, Low RB, Paul RJ, Periasamy M. Loss of $S M-B$ myosin affects muscle shortening velocity and maximal force development. Nat Cell Biol. 2001;3:1025-1029.

66. Morano I, Chai GX, Baltas LG, Lamounier-Zepter $V$, Lutsch $G$, Kott $M$, Haase H, Bader M. Smoothmuscle contraction without smooth-muscle myosin. Nat Cell Biol. 2000;2:371-375.

67. Vamamura $H$, Masuda $H$, Ikeda W, Tokuyama $T$, Takagi $M$, Shibata $N$, Tatsuta M, Takahashi K. Structure and expression of the human SM22alpha gene, assignment of the gene to chromosome 11, and repression of the promoter activity by cytosine DNA methylation. J Biochem (Tokyo). 1997;122:157-167.

68. Zhang JC, Kim S, Helmke BP, Yu WW, Du KL, Lu MM, Strobeck M, Yu Q, Parmacek MS. Analysis of SM22alpha-deficient mice reveals unanticipated insights into smooth muscle cell differentiation and function. Mol Cell Biol. 2001;21:1336-1344.

69. Li L, Miano JM, Cserjesi P, Olson EN. SM22 alpha, a marker of adult smooth muscle, is expressed in multiple myogenic lineages during embryogenesis. Circ Res. 1996;78:188-195.

70. Lawson D, Harrison M, Shapland C. Fibroblast transgelin and smooth muscle SM22alpha are the same protein, the expression of which is down-regulated in many cell lines. Cell Motil Cytoskeleton. 1997;38:250-257.

71. Danninger C, Gimona M. Live dynamics of GFP-calponin: isoform-specific modulation of the actin cytoskeleton and autoregulation by C-terminal sequences. J Cell Sci. 2000;113 Pt 21:3725-3736.

72. Matthew JD, Khromov AS, MCDuffie MJ, Somlyo AV, Somlyo AP, Taniguchi S, Takahashi K. Contractile properties and proteins of smooth muscles of a calponin knockout mouse. J Physiol. 2000;529 Pt 3:811-824.

73. Miano JM, Olson EN. Expression of the smooth muscle cell calponin gene marks the early cardiac and smooth muscle cell lineages during mouse embryogenesis. J Biol Chem. 1996;271:7095-7103.

74. Yoshikawa H, Taniguchi SI, Yamamura H, Mori S, Sugimoto M, Miyado K, Nakamura K, Nakao K, Katsuki M, Shibata N, Takahashi K. Mice lacking smooth muscle calponin display increased bone formation that is associated with enhancement of bone morphogenetic protein responses. Genes Cells. 1998:3:685-695.

75. Winder S, Walsh M. Inhibition of the actomyosin MgATPase by chicken gizzard calponin. Prog Clin Biol Res. 1990;327:141-148.

76. Shanahan CM, Cay NR, Metcalfe JC, Weissberg PL. High expression of genes for calcificationregulating proteins in human atherosclerotic plaques. J Clin Invest. 1994;93:2393-2402.

77. Menon C, Chacko S. Expression of smooth muscle caldesmon in developing chicken gizzard. Tissue Cell. 1998;30:118-126.

78. Payne AM, Yue P, Pritchard K, Marston SB. Caldesmon mRNA splicing and isoform expression in mammalian smooth-muscle and non-muscle tissues. Biochem J. 1995;305 (Pt 2):445-450.

79. van der Loop FT, Gabbiani G, Kohnen G, Ramaekers FC, van Eys GJ. Differentiation of smooth muscle cells in human blood vessels as defined by smoothelin, a novel marker for the contractile phenotype. Arterioscler Thromb Vasc Biol. 1997;17:665-671. 
80. van Eys GJ, Voller MC, Timmer ED, Wehrens XH, Small JV, Schalken JA, Ramaekers FC, van der Loop FT. Smoothelin expression characteristics: development of a smooth muscle cell in vitro system and identification of a vascular variant. Cell Struct funct. 1997;22:65-72.

81. Kramer J, Aguirre-Arteta AM, Thiel C, Gross CM, Dietz R, Cardoso MC, Leonhardt H. A novel isoform of the smooth muscle cell differentiation marker smoothelin [see comments]. J Mol Med. 1999;77:294-298.

82. Deruiter MC, Rensen SS, Coolen GP, Hierck BP, Bergwerff M, Debie WM, Gittenberger-De Groot AC, Van Eys GJ. Smoothelin expression during chicken embryogenesis: Detection of an embryonic isoform. Dev Dyn. 2001;221:460-463.

83. Christen T, Bochaton-Piallat $M L$, Neuville $P$, Rensen $S$, Redard $M$, van Eys $G$, Gabbiani G. Cultured porcine coronary artery smooth muscle cells. A new model with advanced differentiation. Circ Res. 1999;85:99-107.

84. Komatsu S, Miyazaki K, Tuft RA, Ikebe M. Translocation of telokin by cGMP signaling in smooth muscle cells. Am J Physiol Cell Physiol. 2002;283:C752-761.

85. Birukov KG, Schavocky JP, Shirinsky VP, Chibalina MV, Van Eldik U, Watterson DM. Organization of the genetic locus for chicken myosin light chain kinase is complex: multiple proteins are encoded and exhibit differential expression and localization. J Cell Biochem. 1998;70:402-413.

86. Herring BP, Smith AF. Telokin expression is mediated by a smooth muscle cell-specific promoter. Am J Physiol. 1996;270:C1656-1665.

87. Herring BP. Smith AF. Telokin expression in A10 smooth muscle cells requires serum response factor. Am J Physiol. 1997;272:C1394-1404.

88. Herring BP, Kriegel AM, Hoggatt $A M$. Identification of Barx $2 b$, a serum response factor-associated homeodomain protein. J Biol Chem. 2001;276:14482-14489.

89. WU X, Haystead TA, Nakamoto RK, Somlyo AV, Somlyo AP. Acceleration of myosin light chain dephosphorylation and relaxation of smooth muscle by telokin. Synergism with cyclic nucleotideactivated kinase. J Biol Chem. 1998;273:11362-11369.

90. Hsieh CM, Yet SF, Layne MD, Watanabe M, Hong AM, Perrella MA, Lee ME. Genomic cloning and promoter analysis of aortic preferentially expressed gene-1. Identification of a vascular smooth muscle-specific promoter mediated by an E box motif. J Biol Chem. 1999;274:14344-14351.

91. Chang DF, Belaguli NS, lyer D, Roberts WB, WU SP, Dong XR, Marx JG, Moore MS, Beckerle MC, Majesky MW, Schwartz RJ. Cysteine-rich LIM-only proteins CRP1 and CRP2 are potent smooth muscle differentiation cofactors. Dev Cell. 2003;4:107-118.

92. Yet SF, Folta SC, Jain MK, Hsieh CM, Maemura K, Layne MD, Zhang D, Marria PB, Yoshizumi M, Chin MT, Perrella MA, Lee ME. Molecular cloning, characterization, and promoter analysis of the mouse Crp2 $/ 5$ mLim gene. Preferential expression of its promoter in the vascular smooth muscle cells of transgenic mice. J Biol Chem. 1998;273:10530-10537.

93. Henderson JR, Macalma T, Brown D, Richardson JA, Olson EN, Beckerle MC. The LIM protein, CRP1, is a smooth muscle marker. Dev Dyn. 1999;214:229-238.

94. Lilly B, Olson EN, Beckerle MC. Identification of a CArG box-dependent enhancer within the cysteine-rich protein 1 gene that directs expression in arterial but not venous or visceral smooth muscle cells. Dev Biol. 2001;240:531-547.

95. Layne MD, Yet SF, Maemura K, Hsieh CM, Bernfield M, Perrella MA, Lee ME. Impaired abdominal wall development and deficient wound healing in mice lacking aortic carboxypeptidase-like protein. Mol Cell Biol. 2001;21:5256-5261.

96. Layne MD, Yet SF, Maemura K, Hsieh CM, Liu X, Ith B, Lee ME, Perrella MA. Characterization of the mouse aortic carboxypeptidase-like protein promoter reveals activity in differentiated and dedifferentiated vascular smooth muscle cells. Circ Res. 2002;90:728-736.

97. Glukhova MA, Frid MG, Koteliansky VE. Phenotypic changes of human aortic smooth muscle cells during development and in the adult vessel. Am J Physiol. 1991;261:78-80.

98. Frid MG, Moiseeva EP, Stenmark KR. Multiple phenotypically distinct smooth muscle cell populations exist in the adult and developing bovine pulmonary arterial media in vivo. Circ Res. 1994;75:669-681. 
99. Glukhova MA, Frid MG, Koteliansky VE. Developmental changes in expression of contractile and cytoskeletal proteins in human aortic smooth muscle. J Biol Chem. 1990;265:13042-13046.

100. Weitzer G, Milner DJ, Kim JU, Bradley A, Capetanaki Y. Cytoskeletal control of myogenesis: a desmin null mutation blocks the myogenic pathway during embryonic stem cell differentiation. Dev Biol. 1995;172:422-439.

101. Sjuve R, Arner A, Li Z, Mies B, Paulin D, Schmittner $M$, Small N. Mechanical alterations in smooth muscle from mice lacking desmin. J Muscle Res Cell Motil. 1998;19:415-429.

102. Osborn M, Caselitz J, Puschel K, Weber $K$. Intermediate filament expression in human vascular smooth muscle and in arteriosclerotic plaques. Virchows Arch A Pathol Anat Histopathol. 1987;411:449-458.

103. Kuisk IR, LiH, Tran D, Capetanaki Y. A single MEF2 site governs desmin transcription in both heart and skeletal muscle during mouse embryogenesis. Dev Biol. 1996;174:1-13.

104. Raguz S, Hobbs C, Yague E, loannou PA, Walsh FS, Antoniou M. Muscle-specific locus control region activity associated with the human desmin gene. Dev Biol. 1998;201:26-42.

105. Gardner H, Kreidberg J, Koteliansky $V$, Jaenisch $R$. Deletion of integrin alpha 1 by homologous recombination permits normal murine development but gives rise to a specific deficit in cell adhesion. Dev Biol. 1996;175:301-313.

106. Yano $H$, Hayashi $K$, Haruna $M$, Sobue $K$. Identification of two distinct promoters in the chicken caldesmon gene. Biochem Biophys Res Commun. 1994;201:618-626.

107. Huber PA. Caldesmon. Int J Biochem Cell Biol. 1997;29:1047-1051.

108. Yoshikai S, Ikebe M. Molecular cloning of the chicken gizzard telokin gene and cDNA. Arch Biochem Biophys. 1992;299:242-247.

109. Schmid E, Osborn M, Rungger-Brandle E, Gabbiani G, Weber K, Franke WW. Distribution of vimentin and desmin filaments in smooth muscle tissue of mammalian and avian aorta. Exp Cell Res. 1982;137:329-340.

110. Johansson B, Eriksson A, Virtanen I, Thornell LE. Intermediate filament proteins in adult human arteries. Anat Rec. 1997;247:439-448.

111. Slack JMW. From Egg to Embryo: Regional Specification in Early Development. New York: Cambridge University Press; 1991.

112. Leahy A, Xiong JW, Kuhnert $F$, Stuhlmann $H$. Use of developmental marker genes to define temporal and spatial patterns of differentiation during embryoid body formation. $J$ Exp Zool. 1999;284:67-81.

113. Drab M, Haller $H$, Bychkov $R$, Erdmann B, Lindschau $C$, Haase $H$, Morano I, Luft FC, Wobus AM. From totipotent embryonic stem cells to spontaneously contracting smooth muscle cells: a retinoic acid and db-cAMP in vitro differentiation model. Faseb J. 1997;11:905-915.

114. Du KL, Ip HS, Li J, Chen M, Dandre F, Yu W, LU MM, Owens GK, Parmacek MS. Myocardin is a critical serum response factor cofactor in the transcriptional program regulating smooth muscle cell differentiation. Mol Cell Biol. 2003;23:2425-2437.

115. LiS, Wang DZ, Wang Z, Richardson JA, Olson EN. The serum response factor coactivator myocardin is required for vascular smooth muscle development. Proc Natl Acad Sci U S A. 2003;100:93669370.

116. Yamashita J, Itoh $H$, Hirashima $M$, Ogawa $M$, Nishikawa S, Yurugi T, Naito M, Nakao K. Flk1positive cells derived from embryonic stem cells serve as vascular progenitors. Nature. 2000;408:92-96.

117. Ema $M$, Faloon $P$, Zhang WJ, Hirashima $M$, Reid $T$, Stanford WL, Orkin S, Choi K, Rossant J. Combinatorial effects of Flk1 and Tal1 on vascular and hematopoietic development in the mouse. Genes Dev. 2003;17:380-393.

118. Thijssen VL, Ausma J, Liu GS, Allessie MA, van Eys GJ, Borgers M. Structural changes of atrial myocardium during chronic atrial fibrillation. Cardiovasc Pathol. 2000;9:17-28.

119. Belaguli NS, Sepulveda $U$, Nigam V, Charron F, Nemer $M$, Schwartz RJ. Cardiac tissue enriched factors serum response factor and GATA-4 are mutual coregulators. Mol Cell Biol. 2000;20:75507558. 
120. Chang PS, Li L, MCAnally J, Olson EN. Muscle specificity encoded by specific serum response factorbinding sites. J Biol Chem. 2001;276:17206-17212.

121. Gittenberger-de Groot AC, DeRuiter MC, BergwerffM, Poelmann RE. Smooth muscle cell origin and its relation to heterogeneity in development and disease. Arterioscler Thromb Vasc Biol. 1999;19:1589-1594.

122. Duplaa $C$, Couffinhal T, Dufourcq $P$, Llanas $B$, Moreau $C$, Bonnet $J$. The integrin very late antigen-4 is expressed in human smooth muscle cell. Involvement of alpha 4 and vascular cell adhesion molecule-1 during smooth muscle cell differentiation. Circ Res. 1997;80:159-169.

123. Hungerford JE, Owens CK, Argraves WS, Little CD. Development of the aortic vessel wall as defined by vascular smooth muscle and extracellular matrix markers. Dev Biol. 1996;178:375-392.

124. Bergwerf M, Verberne ME, DeRuiter MC, Poelmann RE, Gittenberger-de Groot AC. Neural crest cell contribution to the developing circulatory system: implications for vascular morphology? Circ Res. 1998;82:221-231.

125. Rosenquist TH, Beall AC. Elastogenic cells in the developing cardiovascular system. Smooth muscle, nonmuscle, and cardiac neural crest. Ann N Y Acad Sci. 1990;588:106-119.

126. Bergwerff M, DeRuiter MC, Poelmann RE, Gittenberger-de Groot AC. Onset of elastogenesis and downregulation of smooth muscle actin as distinguishing phenomena in artery differentiation in the chick embryo. Anat Embryol (Berl). 1996;194:545-557.

127. Gadson PF, Jr., Rossignol C, McCoy J, Rosenquist TH. Expression of elastin, smooth muscle alphaactin, and c-jun as a function of the embryonic lineage of vascular smooth muscle cells. In Vitro Cell Dev Biol Anim. 1993;29A:773-781.

128. Thieszen SL, Dalton M, Gadson PF, Patterson E, Rosenquist TH. Embryonic lineage of vascular smooth muscle cells determines responses to collagen matrices and integrin receptor expression. Exp Cell Res. 1996;227:135-145.

129. Topouzis S, Majesky MW. Smooth muscle lineage diversity in the chick embryo. Two types of aortic smooth muscle cell differ in growth and receptor-mediated transcriptional responses to transforming growth factor-beta. Dev Biol. 1996;178:430-445.

130. Vrancken Peeters MP, Gittenberger-de Groot AC, Mentink MM, Hungerford JE, Little CD, Poelmann RE. Differences in development of coronary arteries and veins. Cardiovasc Res. 1997;36:101-110.

131. Vrancken Peeters MP, Gittenberger-de Groot AC, Mentink MM, Poelmann RE. Smooth muscle cells and fibroblasts of the coronary arteries derive from epithelial-mesenchymal transformation of the epicardium. Anat Embryol (Berl). 1999;199:367-378.

132. DeRuiter MC, Poelmann RE, VanMunsteren JC, Mironov V, Markwald RR, Gittenberger-de Groot AC. Embryonic endothelial cells transdifferentiate into mesenchymal cells expressing smooth muscle actins in vivo and in vitro. Circ Res. 1997;80:444-451.

133. Nikkari ST, Rantala I, Pystynen P, Nikkari T. Characterization of the phenotype of smooth muscle cells in human fetal aorta on the basis of ultrastructure, immunofluorescence, and the composition of cytoskeletal and cytocontractile proteins. Atherosclerosis. 1988;74:33-40.

134. Kocher O, Skalli $O$, Cerutti D, Gabbiani F, Gabbiani G. Cytoskeletal features of rat aortic cells during development. An electron microscopic, immunohistochemical, and biochemical study. Circ Res. 1985;56:829-838.

135. Olivetti $G$, Anversa P, Melissari M, Loud AV. Morphometric study of early postnatal development of the thoracic aorta in the rat. Circ Res. 1980;47:417-424.

136. Shanahan CM, Weissberg PL. Smooth muscle cell heterogeneity: patterns of gene expression in vascular smooth muscle cells in vitro and in vivo. Arterioscler Thromb Vasc Biol. 1998;18:333-338.

137. Gabella G. Development of visceral smooth muscle. Results Probl Cell Differ. 2002;38:1-37.

138. Kofuji $T$, Inoue A. Differentiation of smooth muscle cells in chicken gizzard. Self-catalytic mechanism for the production of smooth muscle cells. Cell Tissue Res. 2002;307:211-223.

139. Smeulders N, Woolf AS, Wilcox DT. Extracellular matrix protein expression during mouse detrusor development. J Pediatr Surg. 2003;38:1-12.

140. Ishisaki $A$, Hayashi $H, L i$ A, Imamura $T$. Human umbilical vein endothelium-derived cells retain potential to differentiate into smooth muscle-like cells. J Biol Chem. 2003;278:1303-1309. 
141. Frid MG, Kale VA, Stenmark KR. Mature vascular endothelium can give rise to smooth muscle cells via endothelial-mesenchymal transdifferentiation: in vitro analysis. Circ Res. 2002;90:1189-1196.

142. Kashiwakura $Y$, Katoh $Y$, Tamayose $K$, Konishi $H$, Takaya $N$, Yuhara $S$, Yamada $M$, Sugimoto $K$, Daida $H$. Isolation of bone marrow stromal cell-derived smooth muscle cells by a human SM22alpha promoter: in vitro differentiation of putative smooth muscle progenitor cells of bone marrow. Circulation. 2003;107:2078-2081.

143. Simper D, Stalboerger PG, Panetta C, Wang S, Caplice NM. Smooth muscle progenitor cells in human blood. Circulation. 2002;106:1199-1204.

144. Lundberg MS, Crow MT. Age-related changes in the signaling and function of vascular smooth muscle cells. Exp Gerontol. 1999;34:549-557.

145. Cook CL, Weiser MC, Schwartz PE, Jones CL, Majack RA. Developmentally timed expression of an embryonic growth phenotype in vascular smooth muscle cells. Circ Res. 1994;74:189-196.

146. Weiser-Evans MC, Schwartz PE, Grieshaber NA, Quinn BE, Grieshaber SS, Belknap JK, Mourani PM, Majack RA, Stenmark KR. Novel embryonic genes are preferentially expressed by autonomously replicating rat embryonic and neointimal smooth muscle cells. Circ Res. 2000;87:608-615.

147. Bochaton-Piallat ML, Clowes AW, Clowes MM, Fischer MW, Redard M, Gabbiani F, Gabbiani G. Cultured arterial smooth muscle cells maintain distinct phenotypes when implanted into carotid artery. Arterioscler Thromb Vasc Biol. 2001;21:949-954.

148. Bitar KN. Aging and neural control of the GI tract: V. Aging and gastrointestinal smooth muscle: from signal transduction to contractile proteins. Am / Physiol Gastrointest Liver Physiol. 2003;284:G1-7.

149. Adams $L D$, Lemire JM, Schwartz SM. A systematic analysis of 40 random genes in cultured vascular smooth muscle subtypes reveals a heterogeneity of gene expression and identifies the tight junction gene zonula occludens 2 as a marker of epithelioid "pup" smooth muscle cells and a participant in carotid neointimal formation. Arterioscler Thromb Vasc Biol. 1999;19:2600-2608.

150. Fornieri C, Quaglino D, Jr., Mori $G$. Role of the extracellular matrix in age-related modifications of the rat aorta. Ultrastructural, morphometric, and enzymatic evaluations. Arterioscler Thromb. 1992;12:1008-1016.

151. Gennaro G, Menard C, Giasson E, Michaud SE, Palasis M, Meloche S, Rivard A. Role of p44/p42 MAP kinase in the age-dependent increase in vascular smooth muscle cell proliferation and neointimal formation. Arterioscler Thromb Vasc Biol. 2003;23:204-210.

152. Low RB, White SL, Low ES, Neuville P, Bochaton-Piallat ML, Gabbiani G. Age dependence of smooth muscle myosin expression by cultured rat aortic smooth muscle cells. Differentiation. 1999;65:151-159.

153. Qin M, Zeng Z, Zheng J, Shah PK, Schwartz SM, Adams LD, Sharifi BG. Suppression subtractive hybridization identifies distinctive expression markers for coronary and internal mammary arteries. Arterioscler Thromb Vasc Biol. 2003;23:425-433.

154. Nakamura A, Isoyama S, Goto K. Vessel size-dependent expression of intermediate-sized filaments, calponin, and $h$-caldesmon in smooth muscle cells of human coronary arteries. Heart Vessels. 1999;14:253-261.

155. Frid MG, Aldashev AA, Dempsey EC, Stenmark KR. Smooth muscle cells isolated from discrete compartments of the mature vascular media exhibit unique phenotypes and distinct growth capabilities. Circ Res. 1997;81:940-952.

156. Villaschi S, Nicosia RF, Smith MR. Isolation of a morphologically and functionally distinct smooth muscle cell type from the intimal aspect of the normal rat aorta. Evidence for smooth muscle cell heterogeneity. In Vitro Cell Dev Biol Anim. 1994;30A:589-595.

157. Li S, Sims S, Jiao $Y$, Chow $L H$, Pickering JG. Evidence from a novel human cell clone that adult vascular smooth muscle cells can convert reversibly between noncontractile and contractile phenotypes. Circ Res. 1999;85:338-348.

158. Liaw L, Skinner MP, Raines EW, Ross R, Cheresh DA, Schwartz SM, Giachelli CM. The adhesive and migratory effects of osteopontin are mediated via distinct cell surface integrins. Role of alpha $v$ beta 3 in smooth muscle cell migration to osteopontin in vitro. J Clin Invest. 1995;95:713-724. 
159. Schnapp $L M$, Breuss $J M$, Ramos DM, Sheppard D, Pytela R. Sequence and tissue distribution of the human integrin alpha 8 subunit: $a$ beta 1-associated alpha subunit expressed in smooth muscle cells. J Cell Sci. 1995;108 (Pt 2):537-544.

160. Velling $T$, Collo $G$, Sorokin L, Durbeej $M$, Zhang $H$, Gullberg D. Distinct alpha $7 A$ beta 1 and alpha $7 B$ beto 1 integrin expression patterns during mouse development: alpha $7 A$ is restricted to skeletal muscle but alpha $7 B$ is expressed in striated muscle, vasculature, and nervous system. Dev Dyn. 1996;207:355-371.

161. Cremona $O$, Savoia $P$, Marchisio PC, Gabbiani $G$, Chaponnier $C$. The alpha 6 and beta 4 integrin subunits are expressed by smooth muscle cells of human small vessels: a new localization in mesenchymal cells. J Histochem Cytochem. 1994;42:1221-1228.

162. Gilbertson-Beadling SK, Fisher C. A potential role for $\mathrm{N}$-cadherin in mediating endothelial cellsmooth muscle cell interactions in the rat vasculature. Lab Invest. 1993;69:203-209.

163. Ivanov D, Philippova M, Antropova J, Gubaeva F, lljinskaya O, Tararak E, Bochkov V, Erne P, Resink $T$, Tkachuk $V$. Expression of cell adhesion molecule $T$-cadherin in the human vasculature. Histochem Cell Biol. 2001;115:231-242.

164. Lau CL, Chacko S. Identification of two types of smooth muscle cells from rabbit urinary bladder. Tissue Cell. 1996;28:339-355.

165. Halayko AJ, Camoretti-Mercado B, Forsythe SM, Vieira JE, Mitchell RW, Wylam ME, Hershenson $M B$, Solway J. Divergent differentiation paths in airway smooth muscle culture: induction of functionally contractile myocytes. Am J Physiol. 1999;276:L197-206.

166. Belaguli NS, Zhou W, Trinh TH, Majesky MW, Schwartz RJ. Dominant negative murine serum response factor: alternative splicing within the activation domain inhibits transactivation of serum response factor binding targets. Mol Cell Biol. 1999;19:4582-4591.

167. Engelse MA, Neele JM, van Achterberg TA, van Aken BE, van Schaik RH, Pannekoek $H$, de Vries $C$. Human activin-A is expressed in the atherosclerotic lesion and promotes the contractile phenotype of smooth muscle cells. Circ Res. 1999;85:931-939.

168. Wang D, Chang PS, Wang Z, Sutherland L, Richardson IA, Small E, Krieg PA, Olson EN. Activation of cardiac gene expression by myocardin, a transcriptional cofactor for serum response factor. Cell. 2001;105:851-862.

169. Blank RS, Swartz EA, Thompson MM, Olson EN, Owens GK. A retinoic acid-induced clonal cell line derived from multipotential $P_{19}$ embryonal carcinoma cells expresses smooth muscle characteristics. Circ Res. 1995; 76:742-749.

170. Hirschi KK, Rohovsky SA, D'Amore PA. PDGF, TGF-beta, and heterotypic cell-cell interactions mediate endothelial cell-induced recruitment of $10 \mathrm{~T} 1 / 2$ cells and their differentiation to a smooth muscle fate. J Cell Biol. 1998;141:805-814.

171. Cunha GR, Battle E, Young P, Brody J, Donjacour A, Hayashi N, Kinbara H. Role of epithelialmesenchymal interactions in the differentiation and spatial organization of visceral smooth muscle. Epithelial Cell Biol. 1992;1:76-83.

172. Kato S, Muraishi A, Miyamoto T, Fox JC. Basic fibroblast growth factor regulates extracellular matrix and contractile protein expression independent of proliferation in vascular smooth muscle cells. In Vitro Cell Dev Biol Anim. 1998;34:341-346.

173. Delafontaine P. Growth factors and vascular smooth muscle cell growth responses. Eur Heart J. 1998;19 Suppl G:G18-22.

174. Fisher SA, Ikebe M, Brozovich F. Endothelin-1 alters the contractile phenotype of cultured embryonic smooth muscle cells. Circ Res. 1997;80:885-893.

175. Bascands JL, Girolami JP, Troly M, Escargueil-Blanc I, Nazzal D, Salvayre R, Blaes N. Angiotensin II induces phenotype-dependent apoptosis in vascular smooth muscle cells. Hypertension. 2001;38:1294-1299.

176. Betsholtz C, Karlsson L, Lindahl P. Developmental roles of platelet-derived growth factors. Bioessays. 2001;23:494-507.

177. Lindahl P, Karlsson L, Hellstrom M, Gebre-Medhin S, Willetts K, Heath JK, Betsholtz C. Alveogenesis failure in PDGF-A-deficient mice is coupled to lack of distal spreading of alveolar smooth muscle cell progenitors during lung development. Development. 1997;124:3943-3953. 
178. Hellstrom $M$, Kalen $M$, Lindah/ P, Abramsson A, Betsholtz $C$. Role of PDGF-B and PDGFR-beta in recruitment of vascular smooth muscle cells and pericytes during embryonic blood vessel formation in the mouse. Development. 1999;126:3047-3055.

179. Schatteman GC, Motley ST, Effmann EL, Bowen-Pope DF. Platelet-derived growth factor receptor alpha subunit deleted Patch mouse exhibits severe cardiovascular dysmorphogenesis. Teratology. 1995;51:351-366.

180. Kotani $M$, Fukuda $N$, Ando $H$, Hu WY, Kunimoto S, Saito S, Kanmatsuse K. Chimeric DNA-RNA hammerhead ribozyme targeting PDGF A-chain mRNA specifically inhibits neointima formation in rat carotid artery after balloon injury. Cardiovasc Res. 2003;57:265-276.

181. Deguchi J, Namba T, Hamada $H$, Nakaoka $T$, Abe J, Sato O, Miyata T, Makuuchi M, Kurokawa K, Takuwa Y. Targeting endogenous platelet-derived growth factor B-chain by adenovirus-mediated gene transfer potently inhibits in vivo smooth muscle proliferation after arterial injury. Gene Ther. 1999;6:956-965.

182. Dickson MC, Martin JS, Cousins FM, Kulkarni AB, Karlsson S, Akhurst RJ. Defective haematopoiesis and vasculogenesis in transforming growth factor-beta 1 knock out mice. Development. 1995;121:1845-1854.

183. Sanford $L P$, Ormsby I, Gittenberger-de Groot $A C$, Sariola $H$, Friedman $R$, Boivin GP, Cardell EL, Doetschman T. TGFbeta2 knockout mice have multiple developmental defects that are nonoverlapping with other TGFbeta knockout phenotypes. Development. 1997;124:2659-2670.

184. Kaartinen V, Voncken JW, Shuler C, Warburton D, Bu D, Heisterkamp N, Groffen J. Abnormal lung development and cleft palate in mice lacking TGF-beta 3 indicates defects of epithelialmesenchymal interaction. Nat Genet. 1995;11:415-421.

185. Shah NM, Groves AK, Anderson DJ. Alternative neural crest cell fates are instructively promoted by TGFbeta superfamily members. Cell. 1996;85:331-343.

186. Hautmann MB, Madsen CS, Owens GK. A transforming growth factor beta (TGFbeta) control element drives TGFbeta-induced stimulation of smooth muscle alpha-actin gene expression in concert with two CArG elements. J Biol Chem. 1997;272:10948-10956.

187. Hocevar BA, Howe PH. Analysis of TGF-beta-mediated synthesis of extracellular matrix components. Methods Mol Biol. 2000;142:55-65.

188. Ignotz RA, Massague J. Transforming growth factor-beta stimulates the expression of fibronectin and collagen and their incorporation into the extracellular matrix. J Biol Chem. 1986;261:43374345.

189. Kubota K, Okazaki J, Louie O, Kent KC, Liu B. TGF-beta stimulates collagen (I) in vascular smooth muscle cells via a short element in the proximal collagen promoter. J Surg Res. 2003;109:43-50.

19o. Kanai H, Tanaka $T$, Aihara Y, Takeda S, Kawabata M, Miyazono K, Nagai R, Kurabayashi M. Transforming growth factor-beta/Smads signaling induces transcription of the cell type-restricted ankyrin repeat protein CARP gene through CAGA motif in vascular smooth muscle cells. Circ Res. 2001;88:30-36.

191. Attisano L, Wrana JL. Signal transduction by the TGF-beta superfamily. Science. 2002;296:16461647.

192. Engelse MA, Lardenoye $J H$, Neele JM, Grimbergen JM, De Vries MR, Lamfers ML, Pannekoek $H$, Quax $\mathrm{PH}, \mathrm{De}$ Vries $C$. Adenoviral activin a expression prevents intimal hyperplasia in human and murine blood vessels by maintaining the contractile smooth muscle cell phenotype. Circ Res. 2002;90:1128-1134.

193. Pawlowski JE, Taylor DS, Valentine M, Hail ME, Ferrer P, Kowala MC, Molloy Cl. Stimulation of activin A expression in rat aortic smooth muscle cells by thrombin and angiotensin II correlates with neointimal formation in vivo. J Clin Invest. 1997;100:639-648.

194. de Waard V, van Achterberg TA, Beauchamp NJ, Pannekoek $H$, de Vries $C$. Cardiac ankyrin repeat protein (CARP) expression in human and murine atherosclerotic lesions: activin induces CARP in smooth muscle cells. Arterioscler Thromb Vasc Biol. 2003;23:64-68.

195. Manabe I, Owens GK. Recruitment of serum response factor and hyperacetylation of histones at smooth muscle-specific regulatory regions during differentiation of a novel P19-derived in vitro smooth muscle differentiation system. Circ Res. 2001;88:1127-1134. 
196. Colbert MC, Kirby ML, Robbins J. Endogenous retinoic acid signaling colocalizes with advanced expression of the adult smooth muscle myosin heavy chain isoform during development of the ductus arteriosus. Circ Res. 1996;78:790-798.

197. Neuville P, Bochaton-Piallat ML, Gabbiani G. Retinoids and arterial smooth muscle cells. Arterioscler Thromb Vasc Biol. 2000;20:1882-1888.

198. Tremblay GB, Giguere V. Coregulators of estrogen receptor action. Crit Rev Eukaryot Gene Expr. 2002;12:1-22.

199. Chen S, Gardner DG. Retinoic acid uses divergent mechanisms to activate or suppress mitogenesis in rat aortic smooth muscle cells. J Clin Invest. 1998;102:653-662.

200. Johst U, Betsch A, Wiskirchen J, Schober W, Vonthein R, Rinkert N, Kehlbach R, Claussen CD, Duda SH. All-Trans and 9-cis Retinoid Acids Inhibit Proliferation, Migration, and Synthesis of Extracellular Matrix of Human Vascular Smooth Muscle Cells by Inducing Differentiation In Vitro. $J$ Cardiovasc Pharmacol. 2003;41:526-535.

201. Miano JM, Topouzis S, Majesky MW, Olson EN. Retinoid receptor expression and all-trans retinoic acid-mediated growth inhibition in vascular smooth muscle cells. Circulation. 1996;93:1886-1895.

202. Wakino S, Kintscher U, Kim S, Jackson S, Vin F, Nagpal S, Chandraratna RA, Hsueh WA, Law RE. Retinoids inhibit proliferation of human coronary smooth muscle cells by modulating cell cycle regulators. Arterioscler Thromb Vase Biol. 2001;21:746-751.

203. Miano JM, Kelly LA, Artacho CA, Nuckolls TA, Piantedosi $R$, Blaner WS. all-Trans-retinoic acid reduces neointimal formation and promotes favorable geometric remodeling of the rat carotid artery after balloon withdrawal injury. Circulation. 1998;98:1219-1227.

204. Boerth NJ, Dey NB, Cornwell TL, Lincoln TM. Cyclic GMP-dependent protein kinase regulates vascular smooth muscle cell phenotype. J Vasc Res. 1997;34:245-259.

205. Guo $K$, Andres V, Walsh K. Nitric oxide-induced downregulation of Cdk2 activity and cyclin A gene transcription in vascular smooth muscle cells. Circulation. 1998;97:2066-2072.

206. Lincoln TM, Dey NB, Boerth NJ, Cornwell TL, Soff GA. Nitric oxide--cyclic GMP pathway regulates vascular smooth muscle cell phenotypic modulation: implications in vascular diseases. Acta Physiol Scand. 1998;164:507-515.

207. Michiels C. Endothelial cell functions.J Cell Physiol. 2003;196:430-443.

208. Fang S, Sharma RV, Bhalla RC. Enhanced recovery of injury-caused downregulation of paxillin protein by eNOS gene expression in rat carotid artery. Mechanism of NO inhibition of intimal hyperplasia? Arterioscler Thromb Vasc Biol. 1999;19:147-152.

209. Rudic RD, Shesely EG, Maeda N, Smithies O, Segal SS, Sessa WC. Direct evidence for the importance of endothelium-derived nitric oxide in vascular remodeling. J Clin Invest. 1998;101:731-736.

210. Chaux A, Ruan XM, Fishbein MC, Ouyang Y, Kaul S, Pass JA, Matloff JM. Perivascular delivery of a nitric oxide donor inhibits neointimal hyperplasia in vein grafts implanted in the arterial circulation. J Thorac Cardiovasc Surg. 1998;115:604-612; discussion 612-604.

211. Bundy RE, Marczin N, Birks EF, Chester AH, Yacoub MH. Transplant atherosclerosis: role of phenotypic modulation of vascular smooth muscle by nitric oxide. Gen Pharmacol. 2000;34:73-84.

212. delaTorre A, Schroeder RA, Bartlett ST, Kuo PC. Differential effects of nitric oxide-mediated Snitrosylation on p50 and c-jun DNA binding. Surgery. 1998;124:137-141; discussion 141-132.

213. Nikitovic D, Holmgren A, Spyrou G. Inhibition of AP-1 DNA binding by nitric oxide involving conserved cysteine residues in Jun and Fos. Biochem Biophys Res Commun. 1998;242:109-112.

214. Ishida A, Sasaguri $T$, Kosaka $C$, Nojima $H$, Ogata J. Induction of the cyclin-dependent kinase inhibitor p21(Sdi1/Cip1/Waf1) by nitric oxide-generating vasodilator in vascular smooth muscle cells. J Biol Chem. 1997;272:10050-10057.

215. Koyama H, Raines EW, Bornfeldt KE, Roberts $J M$, Ross R. Fibrillar collagen inhibits arterial smooth muscle proliferation through regulation of Cdk2 inhibitors. Cell. 1996;87:1069-1078.

216. Ichii T, Koyama H, Tanaka S, Kim S, Shioi A, Okuno Y, Raines EW, Iwao H, Otani S, Nishizawa Y. Fibrillar collagen specifically regulates human vascular smooth muscle cell genes involved in cellular responses and the pericellular matrix environment. Circ Res. 2001;88:460-467.

217. Raines EW, Koyama $H$, Carragher NO. The extracellular matrix dynamically regulates smooth muscle cell responsiveness to PDGF. Ann N Y Acad Sci. 2000;902:39-51; discussion 51-32. 
218. Li S, Chow LH, Pickering JG. Cell surface-bound collagenase-1 and focal substrate degradation stimulate the rear release of motile vascular smooth muscle cells. J Biol Chem. 2000;275:3538435392.

219. Hirst SJ, Twort CH, Lee TH. Differential effects of extracellular matrix proteins on human airway smooth muscle cell proliferation and phenotype. Am J Respir Cell Mol Biol. 2000;23:335-344.

220. Thyberg J, Hultgardh-Nilsson A. Fibronectin and the basement membrane components laminin and collagen type $\mathrm{N}$ influence the phenotypic properties of subcultured rat aortic smooth muscle cells differently. Cell Tissue Res. 1994;276:263-271.

221. Hedin U, Bottger BA, Forsberg E, Johansson S, Thyberg J. Diverse effects of fibronectin and laminin on phenotypic properties of cultured arterial smooth muscle cells. J Cell Biol. 1988;107:307-319.

222. Glukhova MA, frid MG, Shekhonin BV, Vasilevskaya TD, Grunwald J, Saginati M, Koteliansky VE. Expression of extra domain A fibronectin sequence in vascular smooth muscle cells is phenotype dependent. J Cell Biol. 1989;109:357-366.

223. Li S, Lao J, Chen BP, Li YS, Zhao Y, Chu J, Chen KD, Tsou TC, Peck K, Chien S. Genomic analysis of smooth muscle cells in 3-dimensional collagen matrix. Faseb J. 2003;17:97-99.

224. Stegemann JP, Nerem RM. Altered response of vascular smooth muscle cells to exogenous biochemical stimulation in two- and three-dimensional culture. Exp Cell Res. 2003;283:146-155.

225. Moiseeva EP. Adhesion receptors of vascular smooth muscle cells and their functions. Cardiovasc Res. 2001;52:372-386.

226. Li J, Zhang YP, Kirsner RS. Angiogenesis in wound repair: angiogenic growth factors and the extracellular matrix. Microsc Res Tech. 2003;60:107-114.

227. DiSandro MJ, Li Y, Baskin LS, Hayward S, Cunha G. Mesenchymal-epithelial interactions in bladder smooth muscle development: epithelial specificity. J Urol. 1998;160:1040-1046; discussion 1079.

228. Vang Y, Relan NK, Przywara DA, Schuger L. Embryonic mesenchymal cells share the potential for smooth muscle differentiation: myogenesis is controlled by the cell's shape. Development. 1999;126:3027-3033.

229. Parmacek MS. Transcriptional programs regulating vascular smooth muscle cell development and differentiation. Curr Top Dev Biol. 2001;51:69-89.

230. Hu N, Clark EB. Hemodynamics of the stage 12 to stage 29 chick embryo. Circ Res. 1989;65:16651670.

231. O'Callaghan C, Williams B. Mechanical strain-induced extracellular matrix production by human vascular smooth muscle cells: role of TGF-beta(1). Hypertension. 2000;36:319-324.

232. Upadhyay J, Aitken KJ, Damdar C, Bolduc S, Bagli DJ. Integrins expressed with bladder extracellular matrix after stretch injury in vivo mediate bladder smooth muscle cell growth in vitro. $J$ Urol. 2003;169:750-755.

233. Reusch HP, Chan $G$, Ives HE, Nemenoff RA. Activation of JNK/SAPK and ERK by mechanical strain in vascular smooth muscle cells depends on extracellular matrix composition. Biochem Biophys Res Commun. 1997;237:239-244.

234. Birukov KG, Bardy N, Lehoux S, Merval R, Shirinsky VP, Tedgui A. Intraluminal pressure is essential for the maintenance of smooth muscle caldesmon and filamin content in aortic organ culture. Arterioscler Thromb Vasc Biol. 1998;18:922-927.

235. Lehoux S, Tedgui A. Signal transduction of mechanical stresses in the vascular wall. Hypertension. 1998;32:338-345.

236. Reusch $P$, Wagdy-H, Reusch $R$, Wilson $E$, Ives HE. Mechanical strain increases smooth muscle and decreases nonmuscle myosin expression in rat vascular smooth muscle cells. Circ Res. 1996;79:1046-1053.

237. Tock J, Van Putten V, Stenmark KR, Nemenoff RA. Induction of SM-alpha-actin expression by mechanical strain in adult vascular smooth muscle cells is mediated through activation of $J N K$ and p38 MAP kinase. Biochem Biophys Res Commun. 2003;301:1116-1121.

238. Birukov KG, Shirinsky VP, Stepanova OV, Tkachuk VA, Hahn AW, Resink TJ, Smirnov VN. Stretch affects phenotype and proliferation of vascular smooth muscle cells. Mol Cell Biochem. 1995;144:131-139. 
239. Hipper A, Isenberg G. Cyclic mechanical strain decreases the DNA synthesis of vascular smooth muscle cells. Pflugers Arch. 2000;440:19-27.

240. Jain MK, Layne MD, Watanabe $M$, Chin MT, Feinberg MW, Sibinga NE, Hsieh CM, Yet SF, Stemple $D L$, Lee ME. In vitro system for differentiating pluripotent neural crest cells into smooth muscle cells. J Biol Chem. 1998;273:5993-5996.

241. Suzuki $T$, Kim HS, Kurabayashi $M$, Hamada $H$, Fujii $H$, Aikawa $M$, Watanabe $M$, Watanabe $N$, Sakomura $Y$, Yazaki $Y$, Nagai R. Preferential differentiation of $P 19$ mouse embryonal carcinoma cells into smooth muscle cells. Use of retinoic acid and antisense against the central nervous system-specific POU transcription factor Brn-2. Circ Res. 1996; 78:395-404.

242. Yoshida T, Sinha S, Dandre F, Wamhoff BR, Hoofnagle MH, Kremer BE, Wang DZ, Olson EN, Owens $G K$. Myocardin is a key regulator of CArG-dependent transcription of multiple smooth muscle marker genes. Circ Res. 2003;92:856-864.

243. Kimes $B W, B$ randt $B L$. Characterization of two putative smooth muscle cell lines from rat thoracic aorta. Exp Cell Res. 1976;98:349-366.

244. Rao RS, Miano JM, Olson EN, Seidel CL. The A1o cell line: a model for neonatal, neointimal, or differentiated vascular smooth muscle cells? Cardiovasc Res. 1997;36:118-126.

245. Rothman A, Kulik TJ, Taubman MB, Berk BC, Smith CW, Nadal-Ginard B. Development and characterization of a cloned rat pulmonary arterial smooth muscle cell line that maintains differentiated properties through multiple subcultures. Circulation. 1992;86:1977-1986.

246. Firulli $A B$, Han D, Kelly-Roloff $L$, Koteliansky VE, Schwartz SM, Olson EN, Miano JM. A comparative molecular analysis of four rat smooth muscle cell lines. In Vitro Cell Dev Biol Anim. 1998;34:217226.

247. Hasegawa K, Arakawa E, Oda S, Vanai N, Obinata M, Matsuda Y. Novel smooth muscle cell lines from transgenic mice harboring temperature-sensitive SV4O large T-antigen gene. Temperaturedependent expression of smooth muscle myosin heavy chain-1 and calponin genes. $J \mathrm{Mol} \mathrm{Cell}$ Cardiol. 1997;29:2177-2186.

248. Moessler H, Mericskay M, Li Z, Nag/S, Paulin D, Small JV. The SM 22 promoter directs tissue-specific expression in arterial but not in venous or visceral smooth muscle cells in transgenic mice. Development. 1996;122:2415-2425.

249. Sutherland HG, Kearns M, Morgan HD, Headley AP, Morris C, Martin DI, Whitelaw E. Reactivation of heritably silenced gene expression in mice. Mamm Genome. 2000;11:347-355.

250. XU R, Ho YS, Ritchie RP, Li L. Human SM22 alpha BAC encompasses regulatory sequences for expression in vascular and visceral smooth muscles at fetal and adult stages. Am J Physiol Heart Circ Physiol. 2003;284:H1398-1407.

251. Miano JM, Kitchen CM, Chen J, Maltby KM, Kelly LA, Weiler H, Krahe R, Ashworth LK, Garcia E. Expression of human smooth muscle calponin in transgenic mice revealed with a bacterial artificial chromosome. Am J Physiol Heart Circ Physiol. 2002;282:H1793-1803.

252. Li L, Liu Z, Mercer B, Overbeek $P$, Olson EN. Evidence for serum response factor-mediated regulatory networks governing SM22alpha transcription in smooth, skeletal, and cardiac muscle cells. Dev Biol. 1997;187:311-321.

253. Kim S, ip HS, LU MM, Clendenin C, Parmacek MS. A serum response factor-dependent transcriptional regulatory program identifies distinct smooth muscle cell sublineages. Mol Cell Biol. 1997;17:2266-2278.

254. Strobeck M, Kim S, Zhang JC, Clendenin C, Du KL, Parmacek MS. Binding of serum response factor to $C A r G$ box sequences is necessary but not sufficient to restrict gene expression to arterial smooth muscle cells. J Biol Chem. 2001;276:16418-16424.

255. Hoggatt $A M$, Simon $G M$, Herring BP. Cell-specific regulatory modules control expression of genes in vascular and visceral smooth muscle tissues. Circ Res. 2002;91:1151-1159.

256. Smith AF, Bigsby RM, Word RA, Herring BP. A 310-bp minimal promoter mediates smooth muscle cell-specific expression of telokin. Am J Physiol. 1998;274:C1188-1195; discussion C1187.

257. Madsen CS, Regan CP, Hungerford JE, White SL, Manabe I, Owens GK. Smooth muscle-specific expression of the smooth muscle myosin heavy chain gene in transgenic mice requires 5'-flanking and first intronic DNA sequence. Circ Res. 1998;82:908-917. 
258. Manabe 1, Owens GK. CArG elements control smooth muscle subtype-specific expression of smooth muscle myosin in vivo. J Clin Invest. 2001;107:823-834.

259. Regan CP, Manabe 1, Owens GK. Development of a smooth muscle-targeted cre recombinase mouse reveals novel insights regarding smooth muscle myosin heavy chain promoter regulation. Circ Res. 2000;87:363-369.

260. Franz WM, Mueller OJ, Fleischmann M, Babij P, Frey N, Mueller M, Besenfelder U, Moorman AF, Brem $C$, Katus HA. The $2.3 \mathrm{~kb}$ smooth muscle myosin heavy chain promoter directs gene expression into the vascular system of transgenic mice and rabbits. Cardiovasc Res. 1999;43:10401048.

261. Mack CP, Owens $G K$. Regulation of smooth muscle alpha-actin expression in vivo is dependent on CArG elements within the $S^{\prime}$ and first intron promoter regions. Circ Res. 1999;84:852-861.

262. Kumar MS, Hendrix JA, Johnson AD, Owens GK. Smooth muscle alpha-actin gene requires two Eboxes for proper expression in vivo and is a target of class / basic helix-loop-helix proteins. Circ Res. 2003;92:840-847.

263. Arsenian $S$, Weinhold B, Oelgeschlager $M, R$ Ruther $U$, Nordheim A. Serum response factor is essential for mesoderm formation during mouse embryogenesis. Embo J. 1998;17:6289-6299.

264. Morrisey EE, Tang Z, Sigrist $K$, LU MM, Jiang F, IP HS, Parmacek MS. GATA6 regulates HNF4 and is required for differentiation of visceral endoderm in the mouse embryo. Genes Dev. 1998;12:35793590.

265. Bi W, Drake CI, Schwarz J. The transcription factor MEF2C-null mouse exhibits complex vascular malformations and reduced cardiac expression of angiopoietin 1 and VECF. DeV Biol. 1999;211:255-267.

266. Wiebel $F F$, Rennekampff $V$, Vintersten $K$, Nordheim A. Generation of mice carrying conditional knockout alleles for the transcription factor SRF. Genesis. 2002;32:124-126.

267. Mack CP, Thompson MM, Lawrenz-Smith S, Owens GK. Smooth muscle alpha-actin CArG elements coordinate formation of a smooth muscle cell-selective, serum response factor-containing activation complex. Circ Res. 2000;86:221-232.

268. Miano JM. Serum response factor: toggling between disparate programs of gene expression. $J \mathrm{Mol}$ Cell Cardiol. 2003;35:577-593.

269. Croissant JD, Kim JH, Eichele G, Goering L, Lough J, Pywes $R$, Schwartz RJ. Avian serum response factor expression restricted primarily to muscle cell lineages is required for alpha-actin gene transcription. Dev Biol. 1996;177:250-264.

270. Belaguli NS, Schildmeyer LA, Schwartz RJ. Organization and myogenic restricted expression of the murine serum response factor gene. A role for autoregulation. J Biol Chem. 1997;272:1822218231.

271. Kemp PR, Metcalfe JC. Four isoforms of serum response factor that increase or inhibit smoothmuscle-specific promoter activity. Biochem J. 2000;345 Pt 3:445-451.

272. Yang $S$, Yates $P R$, Whitmarsh $A$, Davis $R$, Sharrocks $A D$. The regulation of TCF transcription factors by MAP kinase cascades. Biochem Soc Trans. 1997;25:153S.

273. OiU P, $L i L$. Histone acetylation and recruitment of serum responsive factor and CREB-binding protein onto SM22 promoter during SM22 gene expression. Circ Res. 2002;90:858-865.

274. Liu SH, Ma JT, Yueh AY, Lees-Miller SP, Anderson CW, Ng SY. The carboxy/-terminal transactivation domain of human serum response factor contains DNA-activated protein kinase phosphorylation sites. J Biol Chem. 1993;268:21147-21154.

275. Janknecht $R$, Ernst $W H$, Houthaeve $T$, Nordheim A. C-terminal phosphorylation of the serumresponse factor. Eur J Biochem. 1993;216:469-475.

276. Rivera VM, Miranti CK, Misra RP, Ginty DD, Chen RH, Blenis J, Greenberg ME. A growth factorinduced kinase phosphorylates the serum response factor at a site that regulates its DNA-binding activity. Mol Cell Biol. 1993;13:6260-6273.

277. Liu HW, Halayko AJ, Fernandes DJ, Harmon GS, McCauley JA, Kocieniewski P, McConville J, Fu Y, Forsythe SM, Kogut P, Bellam S, Dowell M, Churchill J, Solway J, Remaining A. The RhoA/Rho kinase Pathway Regulates Nuclear Localization of Serum Response Factor. Am J Respir Cell Mol Biol. 2003. 
278. Camoretti-Mercado B, Liu HW, Halayko AJ, Forsythe SM, Kyle JW, Li B, Fu Y, McConville J, Kogut P, Vieira JE, Patel NM, Hershenson MB, Fuchs E, Sinha S, Miano JM, Parmacek MS, Burkhardt JK, Solway J. Physiological control of smooth muscle-specific gene expression through regulated nuclear translocation of serum response factor. J Biol Chem. 2000;275:30387-30393.

279. Wang Z, Wang DZ, Pipes GC, Olson EN. Myocardin is a master regulator of smooth muscle gene expression. Proc Natl Acad Sci U S A. 2003;100:7129-7134.

280. Wang DZ, Li S, Hockemeyer D, Sutherland L, Wang Z, Schratt G, Richardson JA, Nordheim A, Olson EN. Potentiation of serum response factor activity by a family of myocardin-related transcription factors. Proc Natl Acad Sci U S A. 2002;99:14855-14860.

281. Carson JA, Fillmore RA, Schwartz RJ, Zimmer WE. The smooth muscle gamma-actin gene promoter is a molecular target for the mouse bagpipe homologue, $m N k \times 3-1$, and serum response factor. $J$ Biol Chem. 2000;275:39061-39072.

282. Nishida W, Nakamura M, Mori S, Takahashi M, Ohkawa $Y$, Tadokoro S, Yoshida K, Hiwada K, Hayashi $K$, Sobue K. A triad of serum response factor and the GATA and NK families governs the transcription of smooth and cardiac muscle genes. $J$ Biol Chem. 2002;277:7308-7317.

283. Nakamura M, Nishida W, Mori S, Hiwada K, Hayashi K, Sobue K. Transcriptional activation of betatropomyosin mediated by serum response factor and a novel Barx homologue, Barx $1 b$, in smooth muscle cells. I Biol Chem. 2001;276:18313-18320.

284. Ma JT, Ng SY. Muscle homeodomain protein MHox inhibits ternary complex formation at the c-fos serum response element. Biochem Biophys Res Commun. 1994;200:1742-1747.

285. Hautmann MB, Thompson MM, Swartz EA, Olson EN, Owens GK. Angiotensin II-induced stimulation of smooth muscle alpha-actin expression by serum response factor and the homeodomain transcription factor MHox. Circ Res. 1997;81:600-610.

286. Mack CP, Somlyo AV, Hautmann M, Somlyo AP, Owens GK. Smooth muscle differentiation marker gene expression is regulated by RhoA-mediated actin polymerization. $J$ Biol Chem. 2001;276:341347.

287. Sotiropoulos A, Gineitis D, Copeland J, Treisman R. Signal-regulated activation of serum response factor is mediated by changes in actin dynamics. Cell. 1999;98:159-169.

288. Vamakawa T, Tanaka S, Numaguchi K, Yamakawa $Y$, Motley ED, Ichihara S, Inagami $T$. Involvement of Rho-kinase in angiotensin II-induced hypertrophy of rat vascular smooth muscle cells. Hypertension. 2000;35:313-318.

289. Turner BM. Cellular memory and the histone code. Cell. 2002;111:285-291.

290. Lee JS, Galvin KM, See RH, Eckner R, Livingston D, Moran E, Shi Y. Relief of YY1 transcriptional repression by adenovirus E1A is mediated by E1A-associated protein p300. Genes Dev. 1995;9:1188-1198.

291. Galvin KM, Shi Y. Multiple mechanisms of transcriptional repression by W1. Mol Cell Biol. 1997;17:3723-3732.

292. Thomas MJ, Seto E. Unlocking the mechanisms of transcription factor YY1: are chromatin modifying enzymes the key? Gene. 1999;236:197-208.

293. Adam PJ, Regan CP, Hautmann MB, Owens GK. Positive and Negative Acting Kruppel-like Transcription Factors Bind a Transforming Growth Factor Beta Control Element Required for Expression of the Smooth Muscle Cell Differentiation Marker SM22\{alpha\} In Vivo. J Biol Chem. 2000.

294. King KE, lyemere VP, Weissberg PL, Shanahan CM. Kruppel-like factor 4 (KLF4/GKLF) is a target of bone morphogenetic proteins and transforming growth factor beta 1 in the regulation of vascular smooth muscle cell phenotype. J Biol Chem. 2003;278:11661-11669.

295. Watanabe N, Kurabayashi M, Shimomura Y, Kawai-Kowase K, Hoshino Y, Manabe I, Watanabe M, Aikawa $M$, Kuro-o $M$, Suzuki $T$, Yazaki $Y$, Nagai R. BTEB2, a Kruppel-like transcription factor, regulates expression of the SMemb/Nonmuscle myosin heavy chain B (SMemb/NMHC-B) gene. Circ Res. 1999;85:182-191.

296. Nagai R, Suzuki T, Aizawa K, Miyamoto S, Amaki T, Kawai-Kowase K, Sekiguchi Kl, Kurabayashi M. Phenotypic modulation of vascular smooth muscle cells: dissection of transcriptional regulatory mechanisms. Ann N Y Acad Sci. 2001;947:56-66; discussion 66-57. 
297. Shindo T, Manabe I, Fukushima $Y$, Tobe K, Aizawa K, Miyamoto S, Kawai-Kowase $K$, Moriyama $N$, Imai Y, Kawakami H, Nishimatsu H, Ishikawa T, Suzuki T, Morita H, Maemura K, Sata M, Hirata Y, Komukai $M$, Kagechika $H$, Kadowaki $T$, Kurabayashi $M$, Nagai $R$. Kruppel-like zinc-finger transcription factor KLF5/BTEB2 is a target for angiotensin // signaling and an essential regulator of cardiovascular remodeling. Nat Med. 2002;8:856-863.

298. Chen S, Kulik M, Lechleider RJ. Smad proteins regulate transcriptional induction of the SM22alpha gene by TGF-beta. Nucleic Acids Res. 2003;31:1302-1310.

299. Verrecchia F, Mauviel A. Transforming growth factor-beta signaling through the Smad pathway: role in extracellular matrix gene expression and regulation. J Invest Dermatol. 2002;118:211-215.

300. Massari ME, Murre C. Helix-loop-helix proteins: regulators of transcription in eucaryotic organisms. Mol Cell Biol. 2000;20:429-440.

301. Asaumi S, Takemoto $M$, Yokote K, Ridall AL, Butler WT, Fujimoto $M$, Kobayashi K, Kawamura $H$, Take A, Saito $Y$, Mori S. Identification and characterization of high glucose and glucosamine responsive element in the rat osteopontin promoter. J Diabetes Complications. 2003;17:34-38.

302. Johnson AD, Owens GK. Differential activation of the SMalphaA promoter in smooth vs. skeletal muscle cells by bHLH factors. Am J Physiol. 1999;276:C1420-1431.

303. Firulli $A B$, Miano JM, Bi W, Johnson AD, Casscells W, Olson EN, Schwarz J. Myocyte enhancer binding factor-2 expression and activity in vascular smooth muscle cells. Association with the activated phenotype. Circ Res. 1996;78:196-204.

304. Lin Q, LU J, Yanagisawa H, Webb R, Lyons GE, Richardson JA, Olson EN. Requirement of the MADSbox transcription factor MEF2C for vascular development. Development. 1998;125:4565-4574.

305. Yamashita J, Itoh H, Ogawa Y, Tamura N, Takaya K, Igaki T, Doi K, Chun TH, Inoue M, Masatsugu K, Nakao $K$. Opposite regulation of Gax homeobox expression by angiotensin II and C-type natriuretic peptide. Hypertension. 1997;29:381-387.

306. Weir L, Chen D, Pastore C, Isner JM, Walsh K. Expression of gax, a growth arrest homeobox gene, is rapidly down-regulated in the rat carotid artery during the proliferative response to balloon injury. J Biol Chem. 1995;270:5457-5461.

307. Smith RC, Branellec D, Gorski DH, Guo K, Perlman H, Dedieu JF, Pastore C, Mahfoudi A, Denefle P, Isner JM, Walsh K. p21CIP1-mediated inhibition of cell proliferation by overexpression of the gax homeodomain gene. Genes Dev. 1997;11:1674-1689.

308. Witzenbichler B, Kureishi Y, Luo Z, Le Roux A, Branellec D, Walsh K. Regulation of smooth muscle cell migration and integrin expression by the Gax transcription factor. $J$ Clin Invest. 1999;104:1469-1480.

309. Andres V, Fisher S, Wearsch P, Walsh K. Regulation of Gax homeobox gene transcription by a combination of positive factors including myocyte-specific enhancer factor 2. Mol Cell Biol. 1995;15:4272-4281.

310. Morrisey EE, Ip HS, LU MM, Parmacek MS. GATA-6: a zinc finger transcription factor that is expressed in multiple cell lineages derived from lateral mesoderm. Dev Biol. 1996;177:309-322.

311. Suzuki E, Evans T, Lowry J, Truong L, Bell DW, Testa JR, Walsh K. The human GATA-6 gene: structure, chromosomal location, and regulation of expression by tissue-specific and mitogenresponsive signals. Genomics. 1996;38:283-290.

312. Perlman H, Suzuki E, Simonson M, Smith RC, Walsh K. GATA-6 induces p21(Cip1) expression and G1 cell cycle arrest. J Biol Chem. 1998;273:13713-13718.

313. Wada $H$, Hasegawa K, Morimoto $T$, Kakita $T$, Yanazume $T$, Sasayama S. A p300 protein as a coactivator of GATA-6 in the transcription of the smooth muscle-myosin heavy chain gene. $J$ Biol Chem. 2000;275:25330-25335.

314. Abe M, Hasegawa K, Wada H, Morimoto T, Yanazume T, Kawamura T, Hirai M, Furukawa $Y, K i t a ~ T$. GATA-6 is involved in PPARgamma-mediated activation of differentiated phenotype in human vascular smooth muscle cells. Arterioscler Thromb Vasc Biol. 2003;23:404-410.

315. Wada H, Hasegawa K, Morimoto T, Kakita T, Yanazume T, Abe M, Sasayama S. Calcineurin-GATA-6 pathway is involved in smooth muscle-specific transcription. J Cell Biol. 2002;156:983-991.

316. Ohkawa $Y$, Hayashi $K$, Sobue $K$. Calcineurin-mediated pathway involved in the differentiated phenotype of smooth muscle cells. Biochem Biophys Res Commun. 2003;301:78-83. 
317. Hill-Eubanks DC, Gomez MF, Stevenson AS, Nelson MT. NFAT regulation in smooth muscle. Trends Cardiovasc Med. 2003;13:56-62.

318. Biesiada E, Hamamori Y, Kedes L, Sartorelli V. Myogenic basic helix-loop-helix proteins and Sp1 interact as components of a multiprotein transcriptional complex required for activity of the human cardiac alpha-actin promoter. Mol Cell Biol. 1999;19:2577-2584.

319. Watanabe $M$, Sakomura Y, Kurabayashi M, Manabe I, Aikawa M, Kuro-o M, Suzuki T, Yazaki Y, Nagai R. Structure and characterization of the 5'-flanking region of the mouse smooth muscle myosin heavy chain (SM1/2) gene. Circ Res. 1996;78:978-989.

320. Jensen DE, Rich CB, Terpstra AJ, Farmer SR, Foster JA. Transcriptional regulation of the elastin gene by insulin-like growth factor-1 involves disruption of $S p 1$ binding. Evidence for the role of $R b$ in mediating Sp1 binding in aortic smooth muscle cells. J Biol Chem. 1995;270:6555-6563.

321. Widom RL, Lee JY, Joseph C, Gordon-Froome I, Korn JH. The hcKrox gene family regulates multiple extracellular matrix genes. Matrix Biol. 2001;20:451-462.

322. Hall MC, Young DA, Waters JG, Rowan AD, Chantry A, Edwards DR, Clark IM. The comparative role of activator protein 1 and Smad factors in the regulation of Timp-1 and MMP-1 gene expression by transforming growth factor-beta 1. J Biol Chem. 2003;278:10304-10313.

323. Carreras I, Rich CB, Panchenko MP, Foster JA. Basic fibroblast growth factor decreases elastin gene transcription in aortic smooth muscle cells. J Cell Biochem. 2002;85:592-600.

324. Ahn JD, Morishita R, Kaneda Y, Lee SJ, Kwon KY, Choi SY, Lee KU, Park JY, Moon U, Park JG, Yoshizumi M, Ouchi Y, Lee IK. Inhibitory effects of novel AP-1 decoy oligodeoxynucleotides on vascular smooth muscle cell proliferation in vitro and neointimal formation in vivo. Circ Res. 2002;90:1325-1332.

325. Watanabe M, Layne MD, Hsieh CM, Maemura K, Gray S, Lee ME, Jain MK. Regulation of smooth muscle cell differentiation by AT-rich interaction domain transcription factors Mrfalpha and Mrf2beta. Circ Res. 2002;91:382-389.

326. Hoggatt AM, Kriegel AM, Smith AF, Herring BP. Hepatocyte nuclear factor-3 homologue 1 (HFH-1) represses transcription of smooth muscle-specific genes. J Biol Chem. 2000;275:31162-31170.

327. Hautmann MB, Adam PJ, Owens CK. Similarities and differences in smooth muscle alpha-actin induction by TGF-beta in smooth muscle versus non-smooth muscle cells. Arterioscler Thromb Vase Biol. 1999;19:2049-2058.

328. Kelm RJ, Jr., Cogan JG, Elder PK, Strauch AR, Getz MJ. Molecular interactions between singlestranded DNA-binding proteins associated with an essential MCAT element in the mouse smooth muscle alpha-actin promoter.J Biol Chem. 1999;274:14238-14245.

329. Carlini LE, Getz MI, Strauch AR, Kelm RJ, Jr. Cryptic MCAT enhancer regulation in fibroblasts and smooth muscle cells. Suppression of TEF-1 mediated activation by the single-stranded DNAbinding proteins, Pur alpha, Pur beta, and MSY1. J Biol Chem. 2002;277:8682-8692.

330. Kelm RJ, Jr., Elder PK, Getz MJ. The single-stranded DNA-binding proteins, puralpha, purbeta, and MSY1 specifically interact with an exon 3-derived mouse vascular smooth muscle alpha-actin messenger RNA sequence [In Process Citation]. J Biol Chem. 1999;274:38268-38275.

331. Dirksen WP, Mohamed SA, Fisher SA. Splicing of a myosin phosphatase targeting subunit 1 alternative exon is regulated by intronic cis-elements and a novel bipartite exonic enhancer/silencer element. J Biol Chem. 2003;278:9722-9732.

332. Wagner EJ, Garcia-Blanco MA. Polypyrimidine tract binding protein antagonizes exon definition. Mol Cell Biol. 2001;21:3281-3288.

333. Roberts $G C$, Gooding $C$, Smith $C W$. Smooth muscle alternative splicing induced in fibroblasts by heterologous expression of a regulatory gene. Embo J. 1996;15:6301-6310.

334. Perez I, Lin CH, MCAfee JG, Patton JG. Mutation of PTB binding sites causes misregulation of alternative 3' splice site selection in vivo. Rna. 1997;3:764-778.

335. Southby J, Gooding C, Smith CW. Polypyrimidine tract binding protein functions as a repressor to regulate alternative splicing of alpha-actinin mutally exclusive exons. Mol Cell Biol. 1999;19:26992711.

336. Gromak N, Smith CW. A splicing silencer that regulates smooth muscle specific alternative splicing is active in multiple cell types. Nucleic Acids Res. 2002;30:3548-3557. 
337. Suzuki $H$, Jin $Y$, Otani $H$, Yasuda $K$, Inoue $K$. Regulation of alternative splicing of alpha-actinin transcript by Bruno-like proteins. Genes Cells. 2002;7:133-141.

338. Rovner AS, Fagnant PM, Lowey S, Trybus KM. The carboxyl-terminal isoforms of smooth muscle myosin heavy chain determine thick filament assembly properties. J Cell Biol. 2002;156:113-123.

339. Lauzon AM, Tyska MJ, Rovner AS, Freyzon Y, Warshaw DM, Trybus KM. A 7-amino-acid insert in the heavy chain nucleotide binding loop alters the kinetics of smooth muscle myosin in the laser trap. $J$ Muscle Res Cell Motil. 1998;19:825-837.

340. Ashton FT, Somlyo AV, Somlyo AP. The contractile apparatus of vascular smooth muscle: intermediate high voltage stereo electron microscopy. J Mol Biol. 1975;98:17-29.

341. VanBuren $P$, Guilford WH, Kennedy $G, W u J$, Warshaw DM. Smooth muscle myosin: a high forcegenerating molecular motor. Biophys J. 1995;68:2565-2585; 2585-259S.

342. de Gasparo M. Angiotensin II and nitric oxide interaction. Heart Fail Rev. 2002;7:347-358.

343. Sanders KM. Invited Review: Mechanisms of calcium handling in smooth muscles. J Appl Physiol. 2001;91:1438-1449.

344. Ganitkevich $V$, Hasse $V$, Pfitzer $G$. Ca2t-dependent and Ca2t-independent regulation of smooth muscle contraction. I Muscle Res Cell Motil. 2002;23:47-52.

345. Somlyo AP, Somlyo AV. Signal transduction and regulation in smooth muscle. Nature. 1994;372:231-236.

346. Feng J, Ito $M$, IChikawa $K$, Isaka $N$, Nishikawa M, Hartshorne DJ, Nakano T. Inhibitory phosphorylation site for Rho-associated kinase on smooth muscle myosin phosphatase. I Biol Chem. 1999;274:37385-37390.

347. Feng J, Ito M, Nishikawa M, Okinaka T, Isaka N, Hartshorne DJ, Nakano T. Dephosphorylation of distinct sites on the $20 \mathrm{kDa}$ myosin light chain by smooth muscle myosin phosphatase. FEBS Lett. 1999;448:101-104.

348. Feng J, Ito $M$, Kureishi $Y$, Ichikawa $K$, Amano $M$, Isaka $N$, Okawa K, Iwamatsu A, Kaibuchi K, Hartshorne DJ, Nakano T. Rho-associated kinase of chicken gizzard smooth muscle. J Biol Chem. 1999;274:3744-3752.

349. MacDonald JA, Borman MA, Muranyi A, Somlyo AV, Hartshorne DJ, Haystead TA. Identification of the endogenous smooth muscle myosin phosphatase-associated kinase. Proc Natl Acad Sci U S A. 2001;98:2419-2424.

350. Muranyi A, MacDonald JA, Deng JT, Wilson DP, Haystead TA, Walsh MP, Erdodi F, Kiss E, Wu Y, Hartshorne DJ. Phosphorylation of the myosin phosphatase target subunit by integrin-linked kinase. Biochem J. 2002;366:211-216.

351. Pfitzer G. Invited review: regulation of myosin phosphorylation in smooth muscle. J Appl Physiol. 2001;91:497-503.

352. Lee MR, Li L, Kitazawa T. Cyclic GMP causes Ca2+ desensitization in vascular smooth muscle by activating the myosin light chain phosphatase. J Biol Chem. 1997;272:5063-5068.

353. Tansey MG, Word RA, Hidaka H, Singer HA, Schworer CM, Kamm KE, Stull JT. Phosphorylation of myosin light chain kinase by the multifunctional calmodulin-dependent protein kinase $I I$ in smooth muscle cells. J Biol Chem. 1992;267:12511-12516.

354. Morgan KG, Gangopadhyay SS. Invited Review: Cross-bridge regulation by thin filament-associated proteins. J Appl Physiol. 2001;91:953-962.

355. Niiro $\mathrm{N}$, Ikebe $\mathrm{M}$. Zipper-interacting protein kinase induces $\mathrm{Ca}(2+)$-free smooth muscle contraction via myosin light chain phosphorylation. J Biol Chem. 2001;276:29567-29574.

356. Kureishi $Y$, Kobayashi S, Amano M, Kimura K, Kanaide H, Nakano T, Kaibuchi K, Ito $M$. Rhoassociated kinase directly induces smooth muscle contraction through myosin light chain phosphorylation. J Biol Chem. 1997;272:12257-12260.

357. Deng JT, Sutherland C, Brautigan DL, Eto $M$, Walsh MP. Phosphorylation of the myosin phosphatase inhibitors, $\mathrm{CPI}-17$ and $\mathrm{PHI}-1$, by integrin-linked kinase. Biochem J. 2002;367:517-524.

358. Carvajal JA, Germain AM, Huidobro-Toro JP, Weiner CP. Molecular mechanism of CGMP-mediated smooth muscle relaxation. J Cell Physiol. 2000;184:409-420.

359. Murphy RA, Walker JS. Inhibitory mechanisms for cross-bridge cycling: the nitric oxide-cGMP signal transduction pathway in smooth muscle relaxation. Acta Physiol Scand. 1998;164:373-380. 
360. Perry SV. Vertebrate tropomyosin: distribution, properties and function. I Muscle Res Cell Motil. 2001;22:5-49.

361. Takahashi $K$, Hiwada K, Kokubu T. Vascular smooth muscle calponin. A novel troponin T-like protein. Hypertension. 1988;11:620-626.

362. Horowitz A, Clement-Chomienne $O$, Walsh MP, Tao T, Katsuyama H, Morgan KG. Effects of calponin on force generation by single smooth muscle cells. Am J Physiol. 1996;270:H1858-1863.

363. Earley 11 , SU X, Moreland RS. Caldesmon inhibits active crossbridges in unstimulated vascular smooth muscle: an antisense oligodeoxynucleotide approach. Circ Res. 1998;83:661-667.

364. Katsuyama H, Wang CL, Morgan KC. Regulation of vascular smooth muscle tone by caldesmon. $J$ Biol Chem. 1992;267:14555-14558.

365. Kudryashov DS, Vorotnikov AV, Dudnakova TV, Stepanova OV, Lukas TI, Sellers JR, Watterson DM, Shirinsky VP. Smooth muscle myosin filament assembly under control of a kinase-related protein (KRP) and caldesmon. J Muscle Res Cell Motil. 2002;23:341-351.

366. Winder SJ, Allen BG, Clement-Chomienne $O$, Walsh MP. Regulation of smooth muscle actin-myosin interaction and force by calponin. Acta Physiol Scand. 1998;164:415-426.

367. Marston S, Burton D, Copeland O, Fraser I, Gao Y, Hodgkinson J, Huber P, Levine B, el-Mezgueldi M, Notarianni $G$. Structural interactions between actin, tropomyosin, caldesmon and calcium binding protein and the regulation of smooth muscle thin filaments. Acta Physiol Scand. 1998;164:401414.

368. Borovikov Yu S, Horiuchi KY, Avrova SV, Chacko S. Modulation of actin conformation and inhibition of actin filament velocity by calponin. Biochemistry. 1996;35:13849-13857.

369. Menice CB, Hulvershorn J, Adam LP, Wang CA, Morgan KG. Calponin and mitogen-activated protein kinase signaling in differentiated vascular smooth muscle. J Biol Chem. 1997;272:2515725161.

370. Leinweber B, Parissenti AM, Gallant C, Gangopadhyay SS, Kinwan-Rhude A, Leavis PC, Morgan KG. Regulation of protein kinase $C$ by the cytoskeletal protein calponin. I Biol Chem. 2000;275:4032940336.

371. Leinweber BD, Fredricksen RS, Hoffman DR, Chalovich JM. Fesselin: a novel synaptopodin-like actin binding protein from muscle tissue. J Muscle Res Cell Motil. 1999;20:539-545.

372. Sohn UD, Cao W, Tang DC, Stull JT, Haeberle JR, Wang CL, Harnett KM, Behar J, Biancani P. Myosin light chain kinase- and PKC-dependent contraction of LES and esophageal smooth muscle. Am J Physiol Gastrointest Liver Physiol. 2001;281:G467-478.

373. Je HD, Gangopadhyay SS, Ashworth TD, Morgan KG. Calponin is required for agonist-induced signal transduction--evidence from an antisense approach in ferret smooth muscle. J Physiol. 2001;537:567-577.

374. Kaneko T, Amano M, Maeda A, Goto H, Takahashi K, Ito $M$, Kaibuchi K. Identification of calponin as a novel substrate of Rho-kinase. Biochem Biophys Res Commun. 2000;273:110-116.

375. Stanier P, Abu-Hayyeh S, Murdoch JN, Eddleston J, Copp AJ. Paralogous sm22alpha (Tagln) genes map to mouse chromosomes 1 and 9: further evidence for a paralogous relationship. Genomics. 1998;51:144-147.

376. Zhang JC, Helmke BP, Shum A, Du K, Yu WW, Lu MM, Davies PF, Parmacek MS. SM22 beta encodes a lineage-restricted gytoskeletal protein with a unique developmentally regulated pattern of expression. Mech Dev. 2002;115:161-166.

377. Fu Y, Liu HW, Forsythe SM, Kogut P, McConville JF, Halayko A, Camoretti-Mercado B, Solway J. Mutagenesis analysis of human SM22: characterization of actin binding. J Appl Physiol. 2000;89:1985-1990.

378. Silver DL, Vorotnikov AV, Watterson DM, Shirinsky VP, Sellers JR. Sites of interaction between kinase-related protein and smooth muscle myosin. J Biol Chem. 1997;272:25353-25359.

379. Krymsky MA, Kudryashov DS, Shirinsky VP, Lukas TJ, Watterson DM, Vorotnikov AV. Phosphorylation of kinase-related protein (telokin) in tonic and phasic smooth muscles. J Muscle Res Cell Motil. 2001;22:425-437. 
380. Sobieszek A, Andruchov OY, Nieznanski K. Kinase-related protein (telokin) is phosphorylated by smooth-muscle myosin light-chain kinase and modulates the kinase activity. Biochem J. 1997;328 (Pt 2):425-430.

381. Nieznanski $K$, Sobieszek A. Telokin (kinase-related protein) modulates the oligomeric state of smooth-muscle myosin light-chain kinase and its interaction with myosin filaments. Biochem $J$. 1997;322 (Pt 1):65-71.

382. MacDonald JA, Walker LA, Nakamoto RK, Gorenne I, Somlyo AV, Somlyo AP, Haystead TA. Phosphorylation of telokin by cyclic nucleotide kinases and the identification of in vivo phosphorylation sites in smooth muscle. FEBS Lett. 2000;479:83-88.

383. Walker LA, MacDonald JA, LiU X, Nakamoto RK, Haystead TA, Somlyo AV, Somlyo AP. Site-specific phosphorylation and point mutations of telokin modulate its Ca2+-desensitizing effect in smooth muscle. J Biol Chem. 2001;276:24519-24524.

384. Tang DD, WU MF, Opazo Saez AM, Gunst SJ. The focal adhesion protein paxillin regulates contraction in canine tracheal smooth muscle. J Physiol. 2002;542:501-513.

385. Marston SB. What is latch? New ideas about tonic contraction in smooth muscle. J Muscle Res Cell Motil. 1989;10:97-100.

386. Khromov A, Somlyo AV, Somlyo AP. MgADP promotes a catch-like state developed through forcecalcium hysteresis in tonic smooth muscle. Biophys J. 1998;75:1926-1934.

387. Wang $Z$, Jiang $H$, Yang $Z$ Q Chacko $S$. Both N-terminal myosin-binding and C-terminal actinbinding sites on smooth muscle caldesmon are required for caldesmon-mediated inhibition of actin filament velocity. Proc Natl Acad Sci U S A. 1997;94:11899-11904.

388. Halayko A, Solway J. Molecular mechanisms of phenotypic plasticity in smooth muscle cells. J Appl Physiol. 2001;90:358-368.

389. Mehta D, Wu MF, Gunst SJ. Role of contractile protein activation in the length-dependent modulation of tracheal smooth muscle force. Am J Physiol. 1996;270:C243-252.

390. Pratusevich VR, Seow CY, Ford LE. Plasticity in canine airway smooth muscle. J Gen Physiol. 1995;105:73-94.

391. Seow CY, Pratusevich VR, Ford LE. Series-to-parallel transition in the filament lattice of ainway smooth muscle. J Appl Physiol. 2000;89:869-876.

392. Fultz ME, Li C, Geng W, Wright $G L$. Remodeling of the actin cytoskeleton in the contracting A7r5 smooth muscle cell. I Muscle Res Cell Motil. 2000;21:775-787.

393. Smith $P G$, Roy C, Zhang YN, Chauduri S. Mechanical stress increases RhoA activation in ainway smooth muscle cells. Am J Respir Cell Mol Biol. 2003;28:436-442.

394. Numaguchi K, Eguchi S, Yamakawa T, Motley ED, Inagami T. Mechanotransduction of rat aortic vascular smooth muscle cells requires RhoA and intact actin filaments. Circ Res. 1999;85:5-11.

395. Hall A. Rho GTPases and the actin cytoskeleton. Science. 1998;279:509-514.

396. Nobes CD, Hall A. Rho, rac, and edc42 GTPases regulate the assembly of multimolecular focal complexes associated with actin stress fibers, lamellipodia, and filopodia. Cell. 1995;81:53-62.

397. Uehata M, Ishizaki T, Satoh H, Ono T, Kawahara T, Morishita T, Tamakawa H, Yamagami K, Inui J, Maekawa M, Narumiya S. Calcium sensitization of smooth muscle mediated by a Rho-associated protein kinase in hypertension. Nature. 1997;389:990-994.

398. Kendrick-Jones J, Smith RC, Craig R, Citi S. Polymerization of vertebrate non-muscle and smooth muscle myosins. J Mol Biol. 1987;198:241-252.

399. Trybus KM. Filamentous smooth muscle myosin is regulated by phosphorylation. I Cell Biol. 1989;109:2887-2894.

400. Sahai E, Alberts AS, Treisman R. RhoA effector mutants reveal distinct effector pathways for cytoskeletal reorganization, SRF activation and transformation. Embo J. 1998;17:1350-1361.

401. Watanabe N, Kato T, Fujita A, Ishizaki T, Narumiya S. Cooperation between mDia1 and ROCK in Rho-induced actin reorganization. Nat Cell Biol. 1999;1:136-143.

402. Ogut $O$, Brozovich FV. Determinants of the contractile properties in the embryonic chicken gizzard and aorta. Am J Physiol Cell Physiol. 2000;279:C1722-1732. 
403. Himpens B, Matthijs G, Somlyo AV, Butler TM, Somlyo AP. Cytoplasmic free calcium, myosin light chain phosphorylation, and force in phasic and tonic smooth muscle. J Gen Physiol. 1988;92:713729.

404. Szymanski PT, Szymanska G, Goyal RK. Differences in calmodulin and calmodulin-binding proteins in phasic and tonic smooth muscles. Am J Physiol Cell Physiol. 2002;282:C94-C104.

405. Matthew JD, Khromov AS, Tybus KM, Somlyo AP, Somlyo AV. Myosin essential light chain isoforms modulate the velocity of shortening propelled by nonphosphorylated cross-bridges. 1 Biol Chem. 1998;273:31289-31296.

406. Huang $Q Q$, Fisher SA, Brozovich FV. Forced expression of essential myosin light chain isoforms demonstrates their role in smooth muscle force production. J Biol Chem. 1999;274:35095-35098.

407. Jacob MP, Badier-Commander $C$, Fontaine $V$, Benazzoug $Y$, Feldman L, Michel JB. Extracellular matrix remodeling in the vascular wall. Pathol Biol (Paris). 2001;49:326-332.

408. Pickering JG. Regulation of vascular cell behavior by collagen : form is function. Circ Res. 2001;88:458-459.

409. Li DY, Brooke B, Davis EC, Mecham RP, Sorensen LK, Boak BB, Eichwald E, Keating MT. Elastin is an essential determinant of arterial morphogenesis. Nature. 1998;393:276-280.

410. Keeley FW, Elmoselhi A, Leenen FH. Enalapril suppresses normal accumulation of elastin and collagen in cardiovascular tissues of growing rats. Am J Physiol. 1992;262:H1013-1021.

411. Laviades C, Varo N, Fernandez J, Mayor G, Gil MJ, Monreal I, Diez J. Abnormalities of the extracellular degradation of collagen type $I$ in essential hypertension. Circulation. 1998;98:535540.

412. Visse $R$, Nagase $H$. Matrix metalloproteinases and tissue inhibitors of metalloproteinases: structure, function, and biochemistry. Circ Res. 2003;92:827-839.

413. Bissell MJ, Hall HG, Parry G. How does the extracellular matrix direct gene expression? $J$ Theor Biol. 1982;99:31-68.

414. Davis MI, Wu X, Nurkiewicz TR, Kawasaki J, Davis GE, Hill MA, Meininger GA. Integrins and mechanotransduction of the vascular myogenic response. Am J Physiol Heart Circ Physiol. 2001;280:H1427-1433.

415. Carey DJ. Syndecans: multifunctional cell-surface co-receptors. Biochem J. 1997;327 (Pt 1):1-16.

416. Schulick AH, Taylor AJ, Zuo W, Qiu CB, Dong G, Woodward RN, Agah R, Roberts AB, Virmani R, Dichek $D A$. Overexpression of transforming growth factor beta1 in arterial endothelium causes hyperplasia, apoptosis, and cartilaginous metaplasia. Proc Natl Acad Sci U S A. 1998;95:69836988.

417. Herdeg C, Oberhoff M, Baumbach A, Schroeder S, Leitritz M, Blattner A, Siegel-Axel DI, Meisner C, Karsch KR. Effects of local all-trans-retinoic acid delivery on experimental atherosclerosis in the rabbit carotid artery. Cardiovasc Res. 2003;57:544-553.

418. Oriandi A, Ehrlich HP, Ropraz P, Spagnoli LG, Gabbiani G. Rat aortic smooth muscle cells isolated from different layers and at different times after endothelial denudation show distinct biological features in vitro. Arterioscler Thromb. 1994;14:982-989.

419. Dartsch PC, Voisard R, Bauriedel G, Hofling B, Betz E. Growth characteristics and cytoskeletal organization of cultured smooth muscle cells from human primary stenosing and restenosing lesions. Arteriosclerosis. 1990;10:62-75.

420. Martinez-Gonzalez J, Berrozpe M, Varela O, Badimon L. Heterogeneity of smooth muscle cells in advanced human atherosclerotic plaques: intimal smooth muscle cells expressing a fibroblast surface protein are highly activated by platelet-released products. Eur I Clin Invest. 2001;31:939949.

421. Chajara $A$, Raoudi $M$, Delpech $B$, Levesque $H$. The fibroproliferative response of arterial smooth muscle cells to balloon catheter injury is associated with increased hyaluronidase production and hyaluronan degradation. Atherosclerosis. 2001;157:293-300.

422. Evanko SP, Angello JC, Wight TN. Formation of hyaluronan-and versican-rich pericellular matrix is required for proliferation and migration of vascular smooth muscle cells. Arterioscler Thromb Vasc Biol. 1999;19:1004-1013. 
423. Majesky MW, Giachelli CM, Reidy MA, Schwartz SM. Rat carotid neointimal smooth muscle cells reexpress a developmentally regulated mRNA phenotype during repair of arterial injury. Circ Res. 1992;71:759-768.

424. Murry CE, Gipaya CT, Bartosek T, Benditt EP, Schwartz SM. Monoclonality of smooth muscle cells in human atherosclerosis. Am J Pathol. 1997;151:697-705.

425. Mano T, Luo Z, Malendowicz SL, Evans T, Walsh K. Reversal of GATA-6 downregulation promotes smooth muscle differentiation and inhibits intimal hyperplasia in balloon-injured rat carotid artery. Circ Res. 1999;84:647-654.

426. Madsen CS, Hershey JC, Hautmann MB, White SL, Owens GK. Expression of the smooth muscle myosin heavy chain gene is regulated by a negative-acting GC-rich element located between two positive-acting serum response factor-binding elements. J Biol Chem. 1997;272:6332-6340.

427. Landry $D B$, Couper LL, Bryant SR, Lindner V. Activation of the NF-kappa B and I kappa B system in smooth muscle cells after rat arterial injury. Induction of vascular cell adhesion molecule-1 and monocyte chemoattractant protein-1. Am J Pathol. 1997;151:1085-1095.

428. Chang MW, Barr E, Lu MM, Barton K, Leiden JM. Adenovirus-mediated over-expression of the cyclin/cyclin-dependent kinase inhibitor, p21 inhibits vascular smooth muscle cell proliferation and neointima formation in the rat carotid artery model of balloon angioplasty. 1 Clin invest. 1995;96:2260-2268.

429. Diez-Juan A, Andres V. Coordinate control of proliferation and migration by the p27Kip1/cyclindependent kinase/retinoblastoma pathway in vascular smooth muscle cells and fibroblasts. Circ Res. 2003;92:402-410.

430. Chen D, Krasinski K, Sylvester A, Chen J, Nisen PD, Andres V. Downregulation of cyclin-dependent kinase 2 activity and cyclin A promoter activity in vascular smooth muscle cells by $227(K I P 1)$, an inhibitor of neointima formation in the rat carotid artery. J Clin Invest. 1997;99:2334-2341.

431. Ihling C, Technau K, Gross V, Schulte-Monting J, Zeiher AM, Schaefer HE. Concordant upregulation of type II-TGF-beta-receptor, the cyclin-dependent kinases inhibitor P27Kip1 and cyclin E in human atherosclerotic tissue: implications for lesion cellularity. Atherosclerosis. 1999;144:7-14.

432. Degertekin $M$, Serruys PW, Foley DP, Tanabe K, Regar E, Vos J, Smits PC, van der Giessen WI, van den Brand $M$, de Feyter $P$, Popma J. Persistent inhibition of neointimal hyperplasia after sirolimuseluting stent implantation: long-term (up to 2 years) clinical, angiographic, and intravascular ultrasound follow-up. Circulation. 2002;106:1610-1613.

433. Engelen JJ, Esterling LE, Albrechts $J C$, Detera-Wadleigh SD, van Eys GJ. Assignment of the human gene for smoothelin (SMTN) to chromosome 22912 by fluorescence in situ hybridization and radiation hybrid mapping. Genomics. 1997;43:245-247.

434. Wehrens XH, Mies B, Gimona M, Ramaekers FC, Van Eys GJ, Small N. Localization of smoothelin in avian smooth muscle and identification of a vascular-specific isoform. FEBS Lett. 1997:405:315320.

435. Golfert F, Kasper M, van Eys GJ, Funk RH. Cytoskeletal characterization of arteriovenous epithelioid cells. Histochem Cell Biol. 1997;108:513-523.

436. Schwartz $S M, O^{\prime} B r i e n E R$, de Blois $D$, Giachelli $C M$. Relevance of smooth muscle replication and development to vascular disease. San Diego, CA: Academic Press, Inc.; 1995.

437. Kocher $O$, Gabbiani $G$. Expression of actin mRNAs in rat aortic smooth muscle cells during development, experimental intimal thickening, and culture. Differentiation. 1986;32:245-251.

438. Skalli O, Bloom WS, Ropraz P, Azzarone B, Gabbiani C. Cytoskeletal remodeling of rat aortic smooth muscle cells in vitro: relationships to culture conditions and analogies to in vivo situations. J Submicrosc Cytol. 1986;18:481-493.

439. Campbell JH, Kocher O, Skalli O, Gabbiani G, Campbell GR. Cytodifferentiation and expression of alpha-smooth muscle actin mRNA and protein during primary culture of aortic smooth muscle cells. Correlation with cell density and proliferative state. Arteriosclerosis. 1989;9:633-643.

440. Thyberg J, Hedin U, Sjolund M, Palmberg L, Bottger BA. Regulation of differentiated properties and proliferation of arterial smooth muscle cells. Arteriosclerosis. 1990;10:966-990. 
441. Birukov KG, Frid MG, Rogers JD, Shirinsky VP, Koteliansky VE, Campbell JH, Campbell GR. Synthesis and expression of smooth muscle phenotype markers in primary culture of rabbit aortic smooth muscle cells: influence of seeding density and media and relation to cell contractility. Exp Cell Res. 1993;204:46-53.

442. Schwartz RS, Edwards WD, Huber KC, Antoniades $L C$, Bailey KR, Camrud AR, Jorgenson MA, Holmes $D R$, Jr. Coronary restenosis: prospects for solution and new perspectives from a porcine model. Mayo Clin Proc. 1993;68:54-62.

443. Zalewski A, Shi Y. Vascular myofibroblasts. Lessons from coronary repair and remodeling. Arterioscler Thromb Vasc Biol. 1997;17:417-422.

444. Gal D, Rongione A, Slovenkai GA, Dejesus ST, Lucas A, Fields CD, Isner JM. Atherosclerotic Yucatan microswine: an animal model with high-grade, fibrocalcific, nonfatty lesions suitable for testing catheter-based interventions. Am Heart J. 1990;119:291-300.

445. Grinstead WC, Rodgers GP, Mazur W, French BA, Cromeens D, Van Pelt C, West SM, Raizner AE. Comparison of three porcine restenosis models: the relative importance of hypercholesterolemia, endothelial abrasion, and stenting. Coron Artery Dis. 1994;5:425-434.

446. Skalli $O$, Ropraz P, Trzeciak A, Benzonana G, Gillessen D, Gabbiani G. A monoclonal antibody against alpha-smooth muscle actin: a new probe for smooth muscle differentiation. $J$ Cell Biol. 1986;103:2787-2796.

447. Benzonana $G$, Skalli $O$, Gabbiani $G$. Correlation between the distribution of smooth muscle or non muscle myosins and alpha-smooth muscle actin in normal and pathological soft tissues. Cell Motil Cytoskeleton. 1988;11:260-274.

448. Serini G, Bochaton-Piallat ML, Ropraz P, Geinoz A, Borsi L, Zardi L, Gabbiani G. The fibronectin domain ED-A is crucial for myofibroblastic phenotype induction by transforming growth factorbeta1. J Cell Biol. 1998;142:873-881.

449. Bradford MM. A rapid and sensitive method for the quantitation of microgram quantities of protein utilizing the principle of protein-dye binding. Anal Biochem. 1976;72:248-254.

450. Ponte $P$, Gunning $P, B l a u H$, Kedes L. Human actin genes are single copy for alpha-skeletal and alpha-cardiac actin but multicopy for beta- and gamma-cytoskeletal genes: $3^{\circ}$ untranslated regions are isotype specific but are conserved in evolution. Mol Cell Biol. 1983;3:1783-1791.

451. Kocher $O$, Gabbiani G. Analysis of alpha-smooth-muscle actin mRNA expression in rat aortic smooth-muscle cells using a specific cDNA probe. Differentiation. 1987;34:201-209.

452. Barja F, Coughlin C, Belin D, Gabbiani G. Actin isoform synthesis and $m R N A$ levels in quiescent and proliferating rat aortic smooth muscle cells in vivo and in vitro. Lab invest. 1986;55:226-233.

453. Rovner AS, Murphy RA, Owens GK. Expression of smooth muscle and nonmuscle myosin heavy chains in cultured vascular smooth muscle cells. J Biol Chem. 1986;261:14740-14745.

454. Babij P, Kawamoto S, White S, Adelstein RS, Periasamy M. Differential expression of SM1 and SM2 myosin isoforms in cultured vascular smooth muscle. Am J Physiol. 1992;262:C607-613.

455. Sartore S, Chiavegato A, Franch R, Faggin E, Pauletto P. Myosin gene expression and cell phenotypes in vascular smooth muscle during development, in experimental models, and in vascular disease. Arterioscler Thromb Vasc Biol. 1997;17:1210-1215.

456. Low RB, White SL. Lung smooth muscle differentiation. Int J Biochem Cell Biol. 1998;30:869-883.

457. Owens GK, Loeb A, Gordon D, Thompson MM. Expression of smooth muscle-specific alpha-isoactin in cultured vascular smooth muscle cells: relationship between growth and cytodifferentiation. $J$ Cell Biol. 1986;102:343-352.

458. Clowes AW, Clowes MM. Kinetics of cellular proliferation after arterial injury. II. Inhibition of smooth muscle growth by heparin. Lab invest. 1985;52:611-616.

459. Desmouliere A, Rubbia-Brandt L, Gabbiani G. Modulation of actin isoform expression in cultured arterial smooth muscle cells by heparin and culture conditions. Arterioscler Thromb. 1991;11:244253.

460. Bjorkerud S. Effects of transforming growth factor-beta 1 on human arterial smooth muscle cells in vitro. Arterioscler Thromb. 1991;11:892-902. 
461. Orlandi A, Ropraz P, Gabbiani G. Proliferative activity and alpha-smooth muscle actin expression in cultured rat aortic smooth muscle cells are differently modulated by transforming growth factorbeta 1 and heparin. Exp Cell Res. 1994;214:528-536.

462. Schwartz RS, Murphy JG, Edwards WD, Camrud AR, Vliestra RE, Holmes DR. Restenosis after balloon angioplasty. A practical proliferative model in porcine coronary arteries. Circulation. 1990;82:2190-2200.

463. Muller DW, Ellis SG, Topol EJ. Experimental models of coronary artery restenosis. J Am Coll Cardiol. 1992;19:418-432.

464. Fager G, Hansson GK, Gown AM, Larson DM, Skalli O, Bondjers G. Human arterial smooth muscle cells in culture: inverse relationship between proliferation and expression of contractile proteins. In Vitro Cell Dev Biol. 1989;25:511-520.

465. Gabbiani G, Schmid E, Winter S, Chaponnier C, de Ckhastonay C, Vandekerckhove J, Weber $K$, Franke WW. Vascular smooth muscle cells differ from other smooth muscle cells: predominance of vimentin filaments and a specific alpha-type actin. Proc Natl Acad Sci U S A. 1981;78:298-302.

466. Borrione AC, Zanellato AM, Scannapieco G, Pauletto P, Sartore S. Myosin heavy-chain isoforms in adult and developing rabbit vascular smooth muscle. Eur J Biochem. 1989;183:413-417.

467. Bochaton-Piallat ML, Gabbiani F, Ropraz P, Gabbiani G. Age influences the replicative activity and the differentiation features of cultured rat aortic smooth muscle cell populations and clones. Arterioscler Thromb. 1993;13:1449-1455.

468. Chamley-Campbell JH, Campbell GR. What controls smooth muscle phenotype? Atherosclerosis. 1981;40:347-357.

469. Castellot JJ, Jr., Favreau LV, Karnovsky MJ, Rosenberg RD. Inhibition of vascular smooth muscle cell growth by endothelial cell-derived heparin. Possible role of a platelet endoglycosidase. J Biol Chem. 1982;257:11256-11260.

470. Weissberg PL, Grainger DJ, Shanahan CM, Metcalfe JC. Approaches to the development of selective inhibitors of vascular smooth muscle cell proliferation. Cardiovasc Res. 1993;27:1191-1198.

471. Kohno M, Yokokawa K, Yasunari K, Minami M, Kano H, Mandal AK, Yoshikawa J. Heparin inhibits human coronary artery smooth muscle cell migration. Metabolism. 1998;47:1065-1069.

472. Koyama N, Kinsella MG, Wight TN, Hedin U, Clowes AW. Heparan sulfate proteoglycans mediate a potent inhibitory signal for migration of vascular smooth muscle cells. Circ Res. 1998;83:305-313.

473. Majack RA, Goodman LV, Dixit VM. Cell surface thrombospondin is functionally essential for vascular smooth muscle cell proliferation. J Cell Biol. 1988;106:415-422.

474. Battegay EJ, Raines EW, Seifert RA, Bowen-Pope DF, Ross R. TGF-beta induces bimodal proliferation of connective tissue cells via complex control of an autocrine PDCF loop. Cell. 1990;63:515-524.

475. Janat MF, Liau G. Transforming growth factor beta 1 is a powerful modulator of platelet-derived growth factor action in vascular smooth muscle cells. J Cell Physiol. 1992;150:232-242.

476. Gollasch M, Haase H, Ried C, Lindschau C, Morano I, Luft FC, Haller H. L-type calcium channel expression depends on the differentiated state of vascular smooth muscle cells. Faseb $\mathrm{J}$. 1998;12:593-601.

477. Neuville P, Yan Z, Gidlof A, Pepper MS, Hansson GK, Gabbiani G, Sirsjo A. Retinoic acid regulates arterial smooth muscle cell proliferation and phenotypic features in vivo and in vitro through an RARalpha-dependent signaling pathway. Arterioscler Thromb Vasc Biol. 1999;19:1430-1436.

478. Bochaton-Piallat ML, Gabbiani G, Pepper MS. Plasminogen activator expression in rat arterial smooth muscle cells depends on their phenotype and is modulated by cytokines. Circ Res. 1998;82:1086-1093.

479. Sanger F, Coulson AR, Barrell BG, Smith AJ, Roe BA. Cloning in single-stranded bacteriophage as an aid to rapid DNA sequencing. J Mol Biol. 1980;143:161-178.

480. Sedmera D, Pexieder T, Vuillemin $M$, Thompson RP, Anderson RH. Developmental patterning of the myocardium. Anat Rec. 2000;258:319-337.

481. Ya J, Markman MW, Wagenaar GT, Blommaart PJ, Moorman AF, Lamers WH. Expression of the smooth-muscle proteins alpha-smooth-muscle actin and calponin, and of the intermediate filament protein desmin are parameters of cardiomyocyte maturation in the prenatal rat heart. Anat Rec. 1997;249:495-505. 
482. Berseth $\mathrm{CL}$. Gestational evolution of small intestine motility in preterm and term infants. $J$ Pediatr. 1989;115:646-651.

483. Gsell $S$, Eschenhagen $T$, Kaspareit $G$, Nose $M$, Scholz $H$, Behrens $O$, Wieland $T$. Apparent upregulation of stimulatory G-protein alpha subunits in the pregnant human myometrium is mimicked by elevated smoothelin expression. Faseb J. 2000;14:17-26.

484. Frid $M G$, Dempsey EC, Durmowicz AG, Stenmark $K R$. Smooth muscle cell heterogeneity in pulmonary and systemic vessels. Importance in vascular disease. Arterioscler Thromb Vasc Biol. 1997;17:1203-1209.

485. Manabe I, Owens GK. The smooth muscle myosin heavy chain gene exhibits smooth muscle subtype-selective modular regulation in vivo. J Biol Chem. 2001;276:39076-39087.

486. Li L, Miano JM, Mercer B, Olson EN. Expression of the SM22alpha promoter in transgenic mice provides evidence for distinct transcriptional regulatory programs in vascular and visceral smooth muscle cells. J Cell Biol. 1996;132:849-859.

487. Gilbert SF. Developmental Biology. Sunderland, MA: Sinauer; 1991.

488. Christ B, Ordahl CP. Early stages of chick somite development. Anat Embryol (Berl). 1995;191:381396.

489. Yablonka-Reuveni Z, Schwartz SM, Christ B. Development of chicken aortic smooth muscle: expression of cytoskeletal and basement membrane proteins defines two distinct cell phenotypes emerging from a common lineage. Cell Mol Biol Res. 1995;41:241-249.

490. Kramer J, Quensel C, Meding J, Cardoso MC, Leonhardt H. Identification and characterization of novel smoothelin isoforms in vascular smooth muscle. I Vasc Res. 2001;38:120-132.

491. van Eys GJMM, De Vries CIM, Rensen SSM, Thijssen VUL, Verkaar ELC, Coolen GPGM, Debie WMH, DeRuiter MC, Wadleigh-Detera SD. Smoothelins: one gene, two proteins, three muscle cell types.... so far. In: Doevendans PA, Reneman RS, van Bilsen M, eds. Cardiovascular specific gene expression. Dordrecht, The Netherlands: Kluwer Academic Publishers; 1999.

492. Kohler $G$, Milstein C. Continuous cultures of fused cells secreting antibody of predefined specificity. Nature. 1975;256:495-497.

493. Laemmli UK. Cleavage of structural proteins during the assembly of the head of bacteriophage T4. Nature. 1970;227:680-685.

494. Auffray $C$, Rougeon F. Purification of mouse immunoglobulin heavy-chain messenger RNAs from total myeloma tumor RNA. Eur J Biochem. 1980;107:303-314.

495. Feinberg AP, Vogelstein B. A technique for radiolabeling DNA restriction endonuclease fragments to high specific activity. Anal Biochem. 1983;132:6-13.

496. Altschul SF, Gish W, Miller W, Myers EW, Lipman DJ. Basic local alignment search tool. J Mol Biol. 1990;215:403-410.

497. Rost B, Sander C. Prediction of protein secondary structure at better than $70 \%$ accuracy. $\mathrm{J} \mathrm{Mol} \mathrm{Biol.}$ 1993;232:584-599.

498. Rost $B$, Sander $C$. Combining evolutionary information and neural networks to predict protein secondary structure. Proteins. 1994;19:55-72.

499. Smith RF, Wiese BA, Wojzynski MK, Davison DB, Worley KC. BCM Search Launcher--an integrated interface to molecular biology data base search and analysis services available on the World Wide Web. Genome Res. 1996;6:454-462.

500. Shimizu RT, Blank RS, Jervis R, Lawrenz-Smith SC, Owens $G K$. The smooth muscle alpha-actin gene promoter is differentially regulated in smooth muscle versus non-smooth muscle cells. I Biol Chem. 1995;270:7631-7643.

501. Rensen $S$, Merkx G, Doevendans P, Geurts Van Kessel A, van Eys G. Structure and chromosome location of Smtn, the mouse smoothelin gene. Cytogenet Cell Genet. 2000;89:225-229.

502. Lees-Miller JP, Heeley DH, Smillie LB. An abundant and novel protein of $22 \mathrm{kDa}(\mathrm{SM22})$ is widely distributed in smooth muscles. Purification from bovine aorta. Biochem J. 1987;244:705-709.

503. Djinovic-Carugo $K$, Young $P$, Gautel $M$, Saraste $M$. Structure of the alpha-actinin rod: molecular basis for cross-linking of actin filaments. Cell. 1999;98:537-546. 
504. Niessen P, Clement S, Fontao L, Chaponnier $C$, Teunissen B, Rensen S, van Eys $G$, Gabbiani $G$. Biochemical evidence for interaction between smoothelin and filamentous actin. Exp Cell Res. 2004; 292:170-178.

505. Gimona $M$, Mital $R$. The single $C H$ domain of calponin is neither sufficient nor necessary for F-actin binding. J Cell Sci. 1998;111 (Pt 13):1813-1821.

506. Van Bokhoven A, Miller $H$, van Eys $G$. Prostatic carcinoma cell lines express an alternative splice variant of the smoothelin gene. Eur Urol. 1998;34:255 (abstract).

507. Ayoubi TA, Van De Ven WJ. Regulation of gene expression by alternative promoters. Faseb $J$. 1996;10:453-460.

508. Miano JM. Mammalian smooth muscle differentiation: origins, markers and transcriptional control. Results Probl Cell Differ. 2002;38:39-59.

509. Bar H, Wende $P$, Watson $L$, Denger $S$, van Eys $G$, Kreuzer J, Jahn L. Smoothelin is an indicator of reversible phenotype modulation of smooth muscle cells in balloon-injured rat carotid arteries. Basic Res Cardiol. 2002;97:9-16.

510. Miano JM, Carlson MJ, Spencer JA, Misra RP. Serum response factor-dependent regulation of the smooth muscle calponin gene. J Biol Chem. 2000;275:9814-9822.

511. Rensen S, Thijssen V, De Vries C, Doevendans P, Detera-Wadleigh S, Van Eys G. Expression of the smoothelin gene is mediated by alternative promoters. Cardiovasc Res. 2002;55:850.

512. Kumar MS, Owens GK. Combinatorial control of smooth muscle-specific gene expression. Arterioscler Thromb Vasc Biol. 2003;23:737-747.

513. Lee TC, Chow KL, Fang P, Schwartz RJ. Activation of skeletal alpha-actin gene transcription: the cooperative formation of serum response factor-binding complexes over positive cis-acting promoter serum response elements displaces a negative-acting nuclear factor enriched in replicating myoblasts and nonmyogenic cells. Mol Cell Biol. 1991;11:5090-5100.

514. Ellis PD, Martin KM, Rickman C, Metcalfe $J C$, Kemp PR. Increased actin polymerization reduces the inhibition of serum response factor activity by Yin Yang 1. Biochem J. 2002;364:547-554.

515. Suijkerbuijk RF, van de Veen $A Y$, van Echten J, Buys $C H$, de Jong B, Oosterhuis JW, Warburton DA, Cassiman J, Schonk D, Geurts van Kessel A. Demonstration of the genuine iso-12p character of the standard marker chromosome of testicular germ cell tumors and identification of further chromosome 12 aberrations by competitive in situ hybridization. Am J Hum Genet. 1991;48:269273.

516. Sambrook J, Fritsch EF, Maniatis T. Molecular cloning: a laboratory manual, second edition. Cold Spring Harbor: Cold Spring Harbor Laboratony Press; 1989.

517. Solovyev VV, Salamov AA, Lawrence CB. Predicting internal exons by oligonucleotide composition and discriminant analysis of spliceable open reading frames. Nucleic Acids Res. 1994;22:51565163.

518. Quandt $K$, Frech $K$, Karas $H$, Wingender $E$, Werner $T$. Matind and Matinspector: new fast and versatile tools for detection of consensus matches in nucleotide sequence data. Nucleic Acids Res. 1995;23:4878-4884.

519. Dunham I, Shimizu N, Roe BA, Chissoe S, Hunt AR, Collins JE, Bruskiewich R, Beare DM, Clamp M, Smink U, Ainscough R, Almeida JP, Babbage A, Bagguley C, Bailey J, Barlow K, Bates KN, Beasley $O$, Bird CP, Blakey S, Bridgeman AM, Buck D, Burgess J, Burrill WD, O'Brien KP, et al. The DNA sequence of human chromosome 22. Nature. 1999;402:489-495.

520. Zilberman A, Dave V, Miano J, Olson EN, Periasamy M. Evolutionarily conserved promoter region containing CArG*-like elements is crucial for smooth muscle myosin heavy chain gene expression. Circ Res. 1998;82:566-575.

521. Coolen GP, DeRuiter MC, Bergwerff $M$, van Eys $G$, van Straaten $H$, Gittenberger-de Groot AC. Expression of smoothelin in the chick embryo. J Mol Cell Cardiol. 1997;29:A42.

522. Goldhamer DJ, Brunk BP, Faerman A, King A, Shani $M$, Emerson $C P, J$. Embryonic activation of the myoD gene is regulated by a highly conserved distal control element. Development. 1995;121:637649.

523. Morano I. Tuning smooth muscle contraction by molecular motors. J Mol Med. 2003;81:481-487. 
524. Quensel C, Kramer J, Cardoso MC, Leonhardt H. Smoothelin contains a novel actin cytoskeleton localization sequence with similarity to troponin T.J Cell Biochem. 2002;85:403-409.

525. van der Zee $R$, Welling GW. A simple method to determine the transit time in mice. $Z$ Versuchstierkd. 1983;25:233-237.

526. Janssen BJ, Leenders PJ, Smits JF. Short-term and long-term blood pressure and heart rate variability in the mouse. Am J Physiol Regul Integr Comp Physiol. 2000;278:R215-225.

527. Raat NJ, Wetzels GE, De Mey JG. Calcium-contraction relationship in rat mesenteric arterial smooth muscle. Effects of exogenous and neurogenic noradrenaline. Pflugers Arch. 1998;436:262269.

528. BuUs $C L$, Pourageaud F, Fazzi GE, Janssen G, Mulvany MJ, De Mey JG. Smooth muscle cell changes during flow-related remodeling of rat mesenteric resistance arteries. Circ Res. 2001;89:180-186.

529. Sugihara K, Muto T, Morioka Y. Motility study in right sided diverticular disease of the colon. Gut. 1983;24:1130-1134.

530. Cortesini C, Pantalone D. Usefulness of colonic motility study in identifying patients at risk for complicated diverticular disease. Dis Colon Rectum. 1991;34:339-342.

531. Trotman IF, Misiewicz J. Sigmoid motility in diverticular disease and the irritable bowel syndrome. Gut. 1988;29:218-222.

532. de Bree E, Grammatikakis J, Christodoulakis $M$, Tsiftsis $D$. The clinical significance of acquired jejunoileal diverticula. Am J Gastroenterol. 1998;93:2523-2528.

533. Chuang $Y H$, Chuang WL, Huang SP, Liu KM, Huang CH. The temporal relationship between the severity of hydroureter and the dynamic changes of obstructed ureters in a rat model. Br J Urol. 1995;76:303-310.

534. Prado IB, dos Santos MH, Lopasso FP, Iriya K, Laudanna AA. Cholestasis in a murine experimental model: lesions include hepatocyte ischemic necrosis. Rev Hosp Clin Fac Med Sao Paulo. 2003;58:2732.

535. Streefkerk JO, de Groot AA, Pfaffendorf $M$, van Zwieten PA. Influence of the nature of precontraction on the responses to commonly employed vasodilator agents in rat-isolated aortic rings. Fundam Clin Pharmacol. 2002;16:485-494.

536. Dorn $G W$, 2nd, Becker MW. Thromboxane A2 stimulated signal transduction in vascular smooth muscle. J Pharmacol Exp Ther. 1993;265:447-456.

537. Cogolludo A, Moreno L, Bosca L, Tamargo J, Perez-Vizcaino F. Thromboxane A2-induced inhibition of voltage-gated $K+$ channels and pulmonary vasoconstriction: role of protein kinase Czeta. Circ Res. 2003;93:656-663.

538. Somlyo AP, Somlyo AV. Signal transduction by G-proteins, rho-kinase and protein phosphatase to smooth muscle and non-muscle myosin II. J Physiol. 2000;522 Pt 2:177-185.

539. Janssen $U, \mathrm{Lu}$-Chao $\mathrm{H}$, Netherton S. Excitation-contraction coupling in pulmonary vascular smooth muscle involves tyrosine kinase and Rho kinase. Am J Physiol Lung Cell Mol Physiol. 2001;280:L666-674.

540. Niu Y, Xu Y, Zhang J, Bai J, Yang H, Ma T. Proliferation and differentiation of prostatic stromal cells. BJU Int. 2001;87:386-393.

541. Ratajska A, Zarska M, Quensel C, Kramer J. Differentiation of the smooth muscle cell phenotypes during embryonic development of coronary vessels in the rat. Histochem Cell Biol. 2001;116:7987.

542. Uranishi R, Baev NI, Kim JH, Awad IA. Vascular smooth muscle cell differentiation in human cerebral vascular malformations. Neurosurgery. 2001;49:671-679; discussion 679-680.

543. Johansson B, Eriksson A, Ramaekers $F$, Thornell LE. Smoothelin and intermediate filament proteins in human aortocoronary saphenous vein by-pass grafts. Histochem J. 1999;31:723-727.

544. Moir LM, Leung SY, Eynott PR, McVicker CG, Ward JP, Chung KF, Hirst SJ. Repeated allergen inhalation induces phenotypic modulation of smooth muscle in bronchioles of sensitized rats. Am J Physiol Lung Cell Mol Physiol. 2003;284:L148-159.

545. Johansson B, Eriksson A, Ramaekers F, Thornell L. Smoothelin in adult and developing human arteries and myocardium. Histochem Cell Biol. 1999;112:291-299. 
546. Hu B, WU Z, Phan SH. Smad3 Mediates Transforming Growth Factor-\{beta\}-Induced \{alpha\}Smooth Muscle Actin Expression. Am J Respir Cell Mol Biol. 2003;29:397-404.

547. Niu YJ, Ma TX, Zhang J, Xu Y, Han RF, Sun G. Androgen and prostatic stroma. Asian J Androl. 2003;5:19-26.

548. Chambers RC, Leoni P, Kaminski N, Laurent GJ, Heller RA. Global expression profiling of fibroblast responses to transforming growth factor-beta1 reveals the induction of inhibitor of differentiation-1 and provides evidence of smooth muscle cell phenotypic switching. Am J Pathol. 2003;162:533-546.

549. Grainger DJ, Witchell CM, Watson JV, Metcalfe JC, Weissberg PL. Heparin decreases the rate of proliferation of rat vascular smooth muscle cells by releasing transforming growth factor beta-like activity from serum. Cardiovasc Res. 1993;27:2238-2247.

550. Fager $G$, Camejo $G$, Bondjers $G$. Heparin-like glycosaminoglycans influence growth and phenotype of human arterial smooth muscle cells in vitro. I. Evidence for reversible binding and inactivation of the platelet-derived growth factor by heparin. In Vitro Cell Dev Biol. 1992;28A:168-175.

551. Hoggatt AM, Kriegel AM, Smith AF, Herring BP. Hepatocyte nuclear factor-3 homologue 1 (HFH-1) represses transcription of smooth muscle-specific genes. J Biol Chem. 2000;275:31162-31170.

552. Dubchak I, Brudno M, Loots GG, Pachter L, Mayor C, Rubin EM, Frazer KA. Active conservation of noncoding sequences revealed by three-way species comparisons. Genome Res. 2000;10:13041306.

553. Mayor C, Brudno M, Schwartz JR, Poliakov A, Rubin EM, Frazer KA, Pachter LS, Dubchak I. VISTA : visualizing global DNA sequence alignments of arbitrary length. Bioinformatics. 2000;16:10461047.

554. Gimona M, Djinovic-Carugo K, Kranewitter WJ, Winder SJ. Functional plasticity of $\mathrm{CH}$ domains. FEBS Lett. 2002;513:98-106.

555. Moores CA, Kendrick-Jones J. Biochemical characterisation of the actin-binding properties of utrophin. Cell Motil Cytoskeleton. 2000;46:116-128.

556. Ayscough KR. In vivo functions of actin-binding proteins. Curr Opin Cell Biol. 1998;10:102-111.

557. De La Cruz EM. Actin-binding proteins: an overview. Results Prob/Cell Differ. 2001;32:123-134.

558. Kannan K, Amariglio N, Rechavi G, Jakob-Hirsch J, Kela I, Kaminski N, Getz G, Domany E, Givol D. DNA microarrays identification of primary and secondary target genes regulated by $p 53$. Oncogene. 2001;20:2225-2234

559. Hao H, Gabbiani G, Bochaton-Piallat ML. Arterial smooth muscle cell heterogeneity: implications for atherosclerosis and restenosis development.Arterioscler Thromb Vasc Biol. 2003 Sep 1;23(9):1510-20. 


\begin{tabular}{|c|c|c|c|}
\hline S'RACE & 5 ' Rapid amplification of CDNA ends & MADS & MCM1-Agamous-Deficiens-Serum \\
\hline ACLP & Aortic carboxypeptidase-like protein & & response factor \\
\hline$\alpha-S M A$ & $\alpha$-Smooth muscle actin & MAP & Mean arterial pressure \\
\hline$A P-1$ & Activator protein-1 & MEF-2 & Myocyte enhancer binding factor- 2 \\
\hline APEG-1 & Aortic preferentially expressed gene-1 & MHC & Myosin heavy chain \\
\hline BAC & Bacterial artificial chromosome & $M L C$ & Myosin light chain \\
\hline b-HLH & Basic-Helix-Loop-Helix & MMP & Matrix metalloproteinase \\
\hline bp & basepairs & MYPT-1 & Myosin phosphatase subunit 1 \\
\hline BTEB2 & $\begin{array}{l}\text { Basic transcriptional element binding } \\
\text { protein-2 }\end{array}$ & $\begin{array}{l}\text { NFATC1 } \\
\text { NFKB }\end{array}$ & $\begin{array}{l}\text { Nuclear factor of activated T cell c1 } \\
\text { Nuclear factor kappa B }\end{array}$ \\
\hline CAMP & Cyclic adenosine monophosphate & $N-M H C$ & Non-muscle myosin heavy chain \\
\hline CARP & Cardiac ankyrin repeat protein & NO & Nitric oxide \\
\hline CAT & Chloramphenicol transferase & ORF & Open reading frame \\
\hline CBP & CREB-binding protein & p & Promoter \\
\hline cGMP & Cyclic guanosine monophosphate & PAC & P1 artificial chromosome \\
\hline $\mathrm{CH}$ & Calponin Homology & PAC1 & Pulmonary artery cell line of the rat \\
\hline CLSM & Confocal laser scan microscopy & PAGE & Polyacrylamide gel electrophoresis \\
\hline cpm & counts per minute & PCAF & P300/CBP-associated factor \\
\hline CRABP & Cellular retinoic acid binding protein & $P C R$ & Polymerase chain reaction \\
\hline CRBP-1 & Cellular retinol binding protein-1 & $\operatorname{PDGF}(R)$ & Platelet derived growth factor (receptor) \\
\hline $\operatorname{CRE}(B)$ & CAMP responsive element (binding) & pgk & Phosphoglycerate kinase \\
\hline Crp & Cysteine rich protein & PHE & Phenylephrine \\
\hline CSA & Cross-sectional area & PKA & CAMP-dependent protein kinase \\
\hline DMEM & Dulbecco's modified Eagle medium & PKC & Protein kinase $C$ \\
\hline E & Embryonic day & PKG & cGMP-dependent protein kinase \\
\hline ECM & Extracellular matrix & PTBP & Polypyrimidine tract binding protein \\
\hline EMSA & Electrophoretic mobility shift assay & PTCA & Percutaneous transluminal coronary \\
\hline eNOS & Endothelial nitric oxide synthase & & angioplasty \\
\hline ES & Embryonic stem & $R A(R)$ & Retinoic acid (receptor) \\
\hline FCS & Fetal calf serum & $\mathrm{RT}$ & Reverse transcriptase \\
\hline FGF & Fibroblast growth factor & $\mathrm{RXR}$ & Retinoid $X$ receptor \\
\hline $\mathrm{FISH}$ & Fluorescence in situ hybridization & SAP & SAF-A/B, Acinus, PIAS \\
\hline FITC & Fluorescein-isothiocyanate & SEM & Standard error of the mean \\
\hline FSP & Fibroblast surface protein & $S M$ & Smooth muscle \\
\hline Gax & Growth-arrest-specific homeobox & SMCs & Smooth muscle cells \\
\hline GKLF & Gut-enriched Krüppel like factor & SMTN & Smoothelin gene \\
\hline HAT & Histone-acetyltransferase & SRF & Serum response factor \\
\hline HDAC & Histone-deacetylases & ssDNA & Single stranded DNA \\
\hline $\mathrm{HFH}-1$ & Hepatocyte nuclear factor-3 homolog 1 & TCE & TGF- $\beta$ control element \\
\hline $\mathrm{HR}$ & Heart rate & TEF-1 & Transcription enhancer factor 1 \\
\hline HUVEC & $\begin{array}{l}\text { Human umbilical vein endothelium- } \\
\text { derived cells }\end{array}$ & $\begin{array}{l}\text { TGF- } \beta \\
\text { TIMP-1 }\end{array}$ & $\begin{array}{l}\text { Transforming growth factor- } \beta \\
\text { Tissue inhibitor of metalloproteinase- } 1\end{array}$ \\
\hline i & Intron & tk & Thymidine kinase \\
\hline$I C_{1}$ & Intronic CArG box of SM-calponin & tRA & All-trans retinoic acid \\
\hline ICAM & Intercellular cell adhesion molecule & TRITC & Tetramethyl-rhodamine-isothiocyanate \\
\hline ISH & In situ hybridization & USF & Upstream stimulatory factor \\
\hline IT & Intimal thickening & VCAM & Vascular cell adhesion molecule \\
\hline kb & kilobase & VEGF & Vascular endothelial growth factor \\
\hline kDa & kilo-Dalton & YAC & Yeast artificial chromosome \\
\hline LAD & Left anterior descending & $Y_{1}$ & Yin Yang-1 \\
\hline
\end{tabular}

